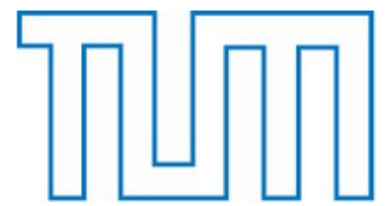

Fakultät für Maschinenwesen

Lehrstuhl für Nukleartechnik

Experimental investigation on the causes for pellet fragmentation under LOCA conditions

Andrea Bianco

Vollständiger Abdruck der von der Fakultät für Maschinenwesen der Technischen Universität München zur Erlangung des akademischen Grades eines

Doktor-Ingenieurs (Dr.-Ing.)

genehmigten Dissertation.

Vorsitzende(r): $\quad$ Univ.-Prof. Dr. Rudolf Neu

Prüfer der Dissertation:

1. Univ.-Prof. Rafael Macián-Juan, Ph.D

2. Prof. Dr. Walter Ambrosini

Universität Pisa, Italien

Die Dissertation wurde am 12. November 2014 bei der Technischen Universität München eingereicht und durch die Fakultät für Maschinenwesen am 23. Juli 2015 angenommen. 



\title{
Dedication
}

\begin{abstract}
A Papá
Wahrlich es ist nicht das Wissen, sondern das Lernen, nicht das Besitzen sondern das Erwerben, nicht das Da-Sein, sondern das Hinkommen, was den größten Genuss gewährt.
\end{abstract}

It is not knowledge, but the act of learning, not possession but the act of getting there, which grants the greatest enjoyment.

\section{Carl Friedrich Gauss}





\section{Abstract}

The study of the Loss Of Coolant Accident (LOCA) in a nuclear reactor has been under investigation since the beginning of the nuclear civil technology. The latest research revealed several new phenomena associated with fuel rod behaviour and the work presented in this dissertation is an experimental research focused on high burnup fuel fragmentation during a LOCA. The research primary objectives are: 1) the design and execution of a LOCA single effects tests to be performed in hot cell environment; 2) the study of fuel fragmentation and fragment size distribution under selected boundary conditions; 3 ) the study of the fission gas release and 4) the development of a semi-empirical correlation to obtain a first order description of the resulting fragments size distribution. The experimental test was designed based on a comprehensive review of past research LOCA tests and observations on fuel fragmentation. A particular attention was dedicated to the study of the IFA-650 LOCA test series performed at the OECD Halden Reactor Project.

The experiment employed the use of Light Water Reactor (LWR) irradiated fuel with a burnup of 52 $\mathrm{MWd} / \mathrm{kgU}$ and the heat-up was simulated with heat provided by an electric oven. The selected boundary conditions were: Rod segment 1: removal of the constraint provided by the cladding (rod ballooning without burst); Rod segment 2: removal of the constraint provided by the cladding and by the rod internal pressure (rod ballooning with burst); Rod segment 3: retain the constraint provided by the cladding (no rod ballooning). The parameters of the rod segments required to obtain the above mentioned boundary conditions were calculated with the fuel performance code TRANSURANUS.

The experiment was successfully performed with the three selected boundary conditions achieved. The post experiment investigations included non-destructive investigation such as neutron radiography, visual inspection and axial diameter measurements by profilometry and destructive investigations such as ceramography of an archive pellet, puncturing, fragments sifting and analysis of the fission gas release.

The neutron radiography showed a fragmentation of a limited number of pellets in correspondence to the burst region of rod segment 2 while the rod segment 1 that only ballooned presented cracks along the entire length. The rod segment 3 was shown to be mostly intact. The fission gas release during the transient was higher for the rod segment $2(7.3 \%)$ followed by the rod segment $1(6 \%)$ and rod segment 3 (3.5\%).

An effort was made to find a law to reproduce the fragment size distribution measured by sifting following the LOCA test. A fractal law correlation was derived starting from the study on the compressing mechanism behavior of concrete. The fractal coefficients were determined as a function of the fuel burnup, and of the individual contribution of the effects studied in this work: the cladding strain and the burst pressure.

The experiment's results and the first modeling approach provide new insights and a better understanding of the mechanism of fuel fragmentation. These outcomes can be used as benchmark for implementing new models in fuel performance codes and to assess the safety significance of this phenomenon considering more appropriate nuclear reactor conditions. 


\section{Zusammenfassung}

Seit Beginn der zivilen Nutzung der Kernenergie wurde der Kühlmittelverlust (KMV) Störfall in einem Leichtwasserreaktor untersucht. Neueste Forschungsergebnisse haben verschiedene Phänomene verbunden mit dem Brennstabverhalten aufgedeckt. Das Ziel dieser Dissertation ist eine experimentelle Untersuchung der Brennstoff-Fragmentierung während eines KMVs. Die Hauptziele sind: 1) die Auslegung und Ausführung eines KMV-Separateffekttests in heißen Zellen; 2) die Untersuchung der Brennstoff-Fragmentierung und die Größenverteilung der Fragmente mit ausgewählten Randbedingungen; 3) die Bewertung der Spaltgasfreisetzung und 4) die Entwicklung einer semiempirischen Korrelation um eine theoretische Beschreibung der Größenverteilung der Fragmente zu erhalten. Das Experiment wurde mit Hilfe einer umfassenden Analyse bisheriger KMV-Experimente und Beobachtungen über die Brennstoff-Fragmentierung geplant. Eine besondere Aufmerksamkeit wurde der Studie der IFA-650 KMV Experimente von dem OECD Halden Reactor Project gewidmet.

Bestrahlte Brennstabsegmente von einem Leichtwasserreaktor mit einem Abbrand von $52 \mathrm{MWd} / \mathrm{kgU}$ wurden in dem Experiment verwendet und die Wärme wurde von einem elektrischen Ofen erzeugt. Die ausgewählten Randbedingungen sind: Brennstabsegment 1: keine Restriktionen durch das Hüllrohr (Dehnung ohne Bersten Hüllrohrs); Brennstabsegment 2: keine Restriktionen durch das Hüllrohr und keine Restriktionen durch den Brennstab-Innendruck (Dehnung und Bersten des Hüllrohrs); Brennstabsegment 3: Restriktion durch das Hüllrohrs (keine Dehnung Hüllrohr). Die Parameter, die erforderlich waren, um die oben genannten Randbedingungen zu erreichen, wurden mit dem Brennstabauslegungsprogram TRANSURANUS berechnet.

Das Experiment wurde mit Erfolg durchgeführt und die drei ausgewählten Randbedingungen wurden erreicht. Nach dem Experiment wurden zerstörungsfreie Untersuchungen wie Neutronradiographie, Sichtprüfung und Messung des Durchmessers mit Profilometrie und Untersuchungen wie Ceramography eines Archivbrennstoffs, Punktierung und Sieben der Fragmente durchgeführt.

Die Neutronradiographie zeigt die Fragmentierung einer begrenzten Zahl der Pellets im Dehnung- und Berst-Bereich von Segment 2 und Risse von Segment 1, wo nur Dehnung auftritt. Die Neutronradiographie von Segment 3 zeigt einen meistens intakten Brennstab. Die Spaltgasfreisetzung während der Transiente war am größten in Segment 2 (7.3\%), gefolgt von Segment 1 (6\%) und Segment $3(3.5 \%)$.

Es wurde eine Korrelation für die Beschreibung der gemessen Größenverteilung der Fragmente entwickelt. Eine Korrelation aus der Theorie der Fraktale wurde von einer Studie des komprimierenden Verhaltens des Betons hergeleitet. Die Fraktal-Koeffizienten wurden als Funktion des Abbrands und der individuellen Beteiligung der zwei analysierten Effekte: Hüllrohr-Dehnung und Bersten-Druck ermittelt.

Die Ergebnisse des Experimentes und der vorgeschlagene Modellierungsansatz bieten neue Einsichten und bessere Erkenntnisse über die Mechanismen für die Brennstoff-Fragmentierung. Die Resultate können als Bezugspunkte für neue Modelle der Brennstoffauslegungsprogramme verwendet werden und um die sicherheitstechnische Bedeutung des Phänomens besser zu bewerten. 


\section{List of Contents}

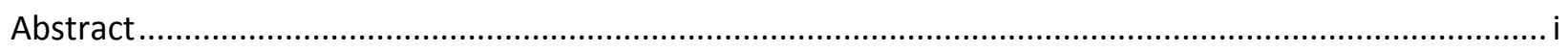

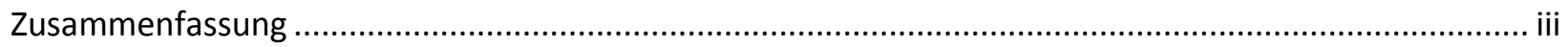

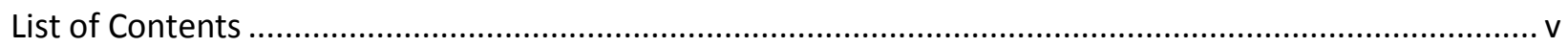

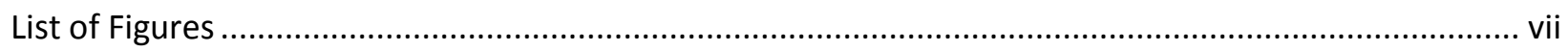

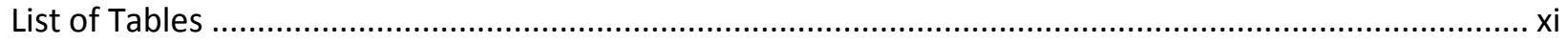

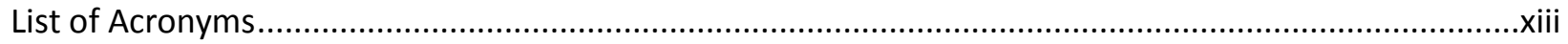

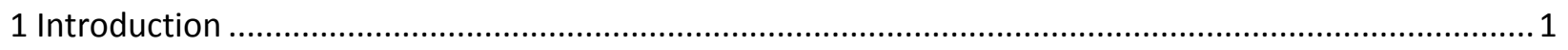

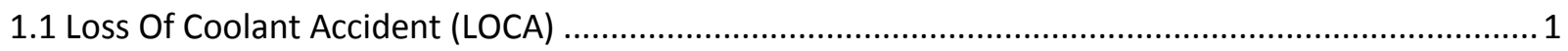

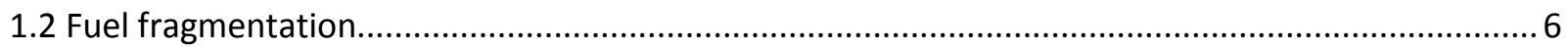

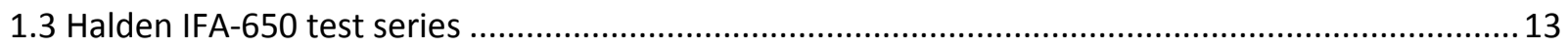

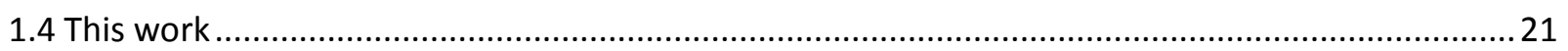

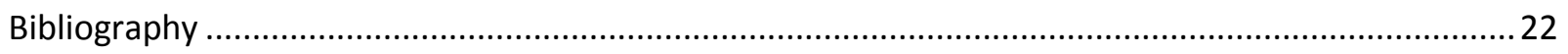

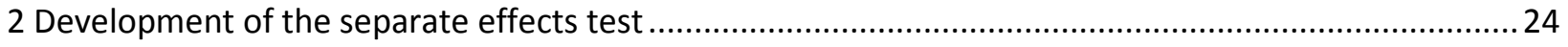

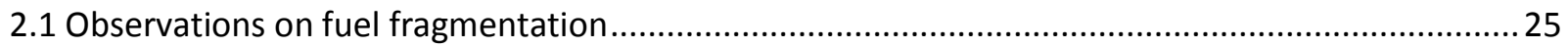

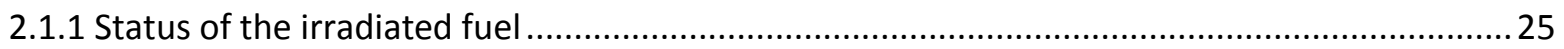

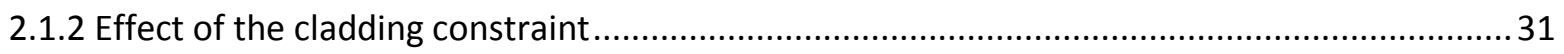

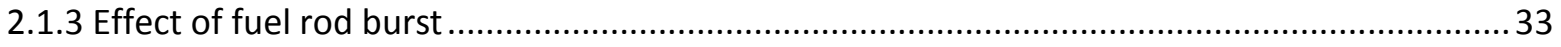

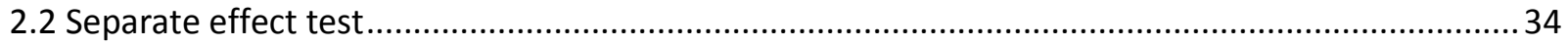

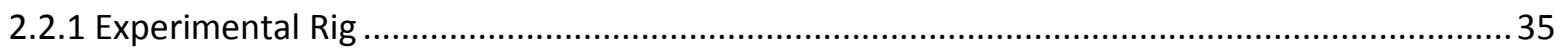

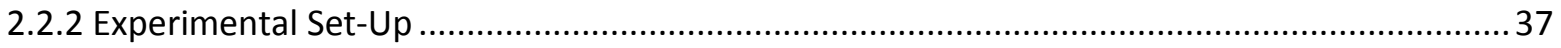

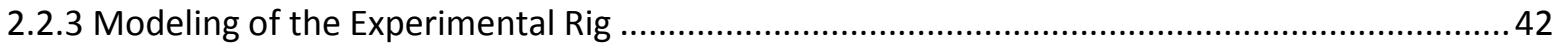

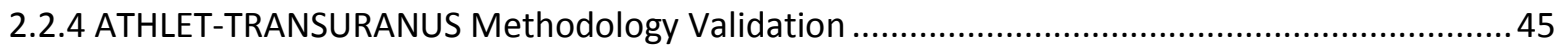

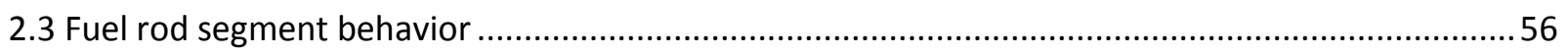

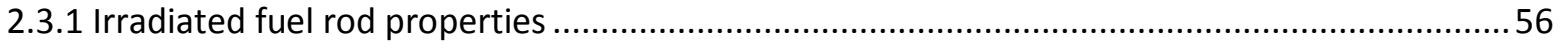

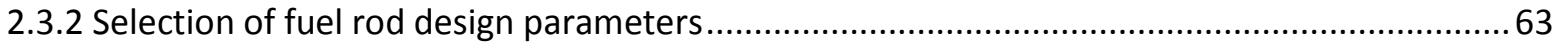

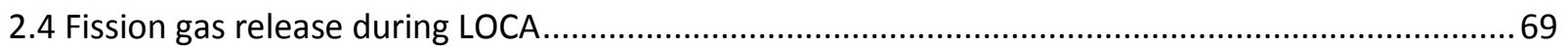

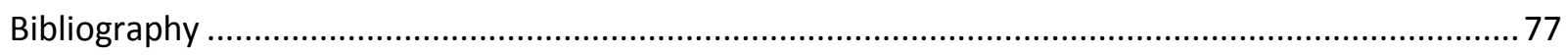

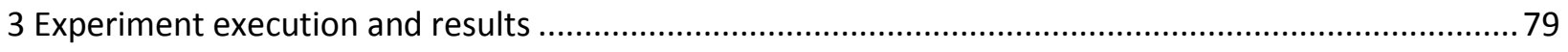

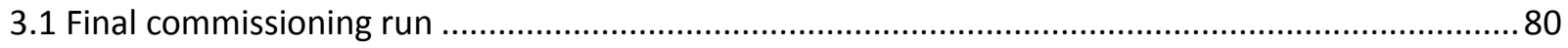




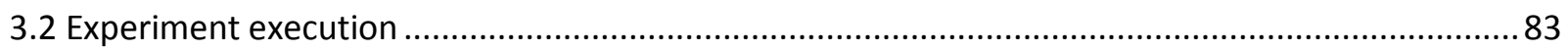

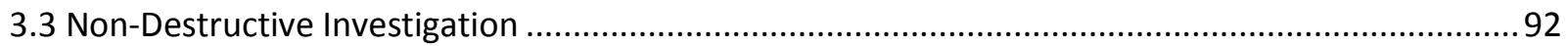

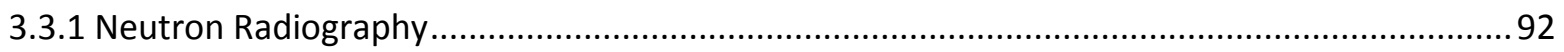

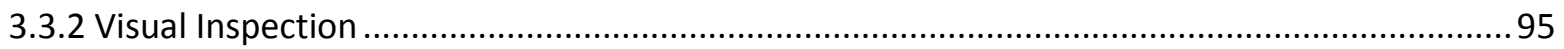

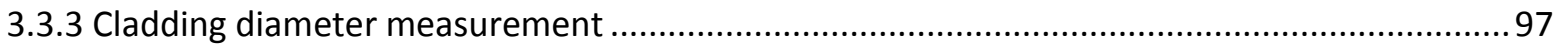

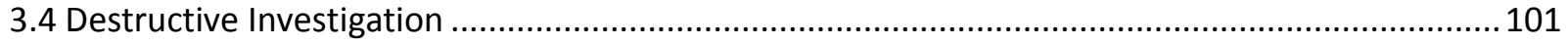

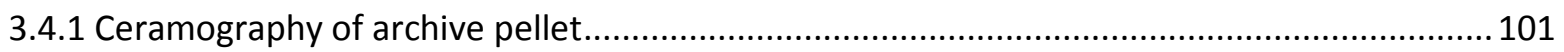

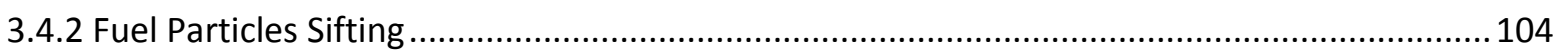

3.4.3 Transient Fission Gas Release ......................................................................................... 107

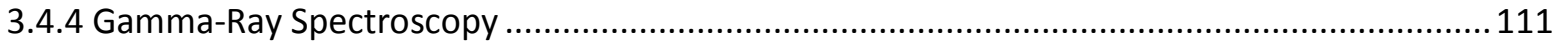

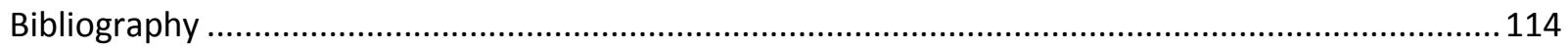

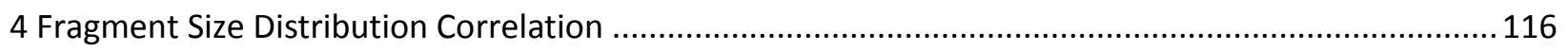

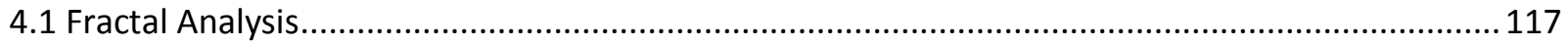

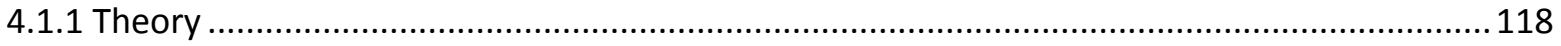

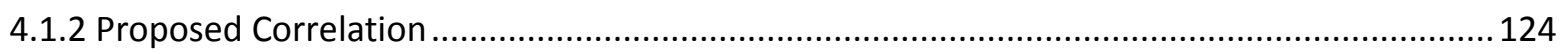

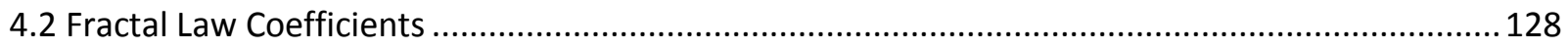

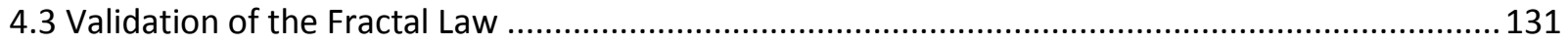

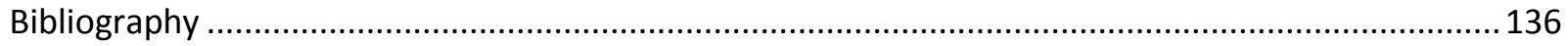

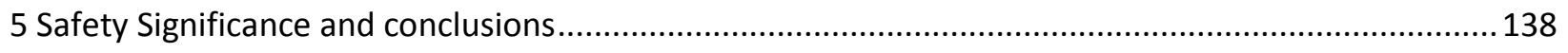

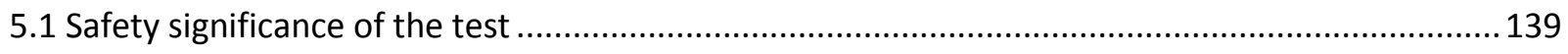

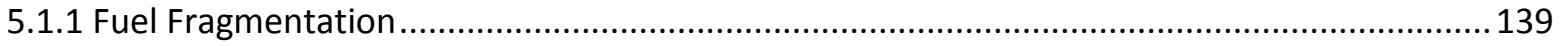

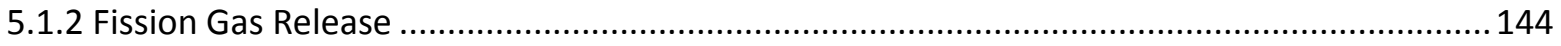

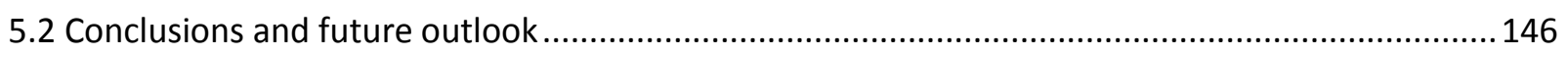

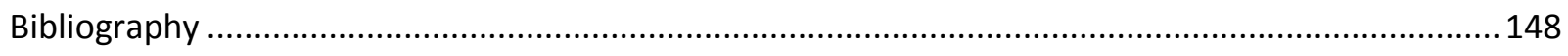

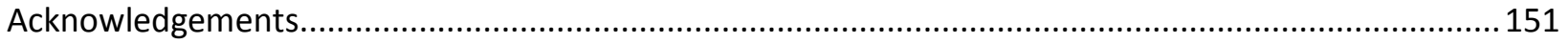




\section{List of Figures}

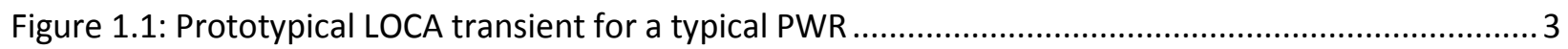

Figure 1.2: Reduction of ductility threshold as function of cladding hydrogen content .......................... 6

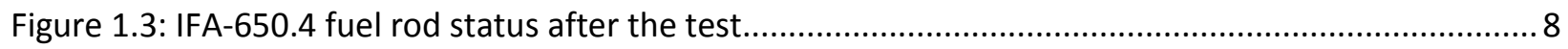

Figure 1.4: NRC Studsvik Test number $193-71 \mathrm{MWd} / \mathrm{kgU}$............................................................ 9

Figure 1.5: NRC Studsvik Test number $198-55 \mathrm{MWd} / \mathrm{kgU}$.............................................................. 9

Figure 1.6: IFA-650 experimental rig and instrumentations.............................................................. 13

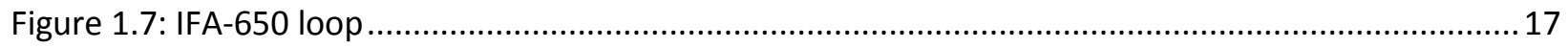

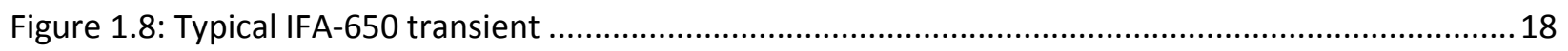

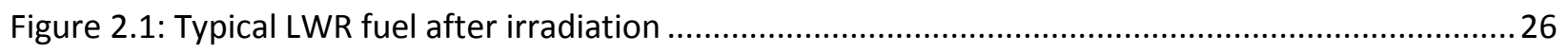

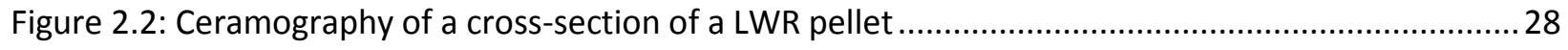

Figure 2.3: Ceramography of pellet's cross-section after LOCA function of BU .....................................29

Figure 2.4: TRANSURANUS simulation of development of "transition" and "fully developed" HBS ..........30

Figure 2.5: IFA-650.5 pellet's cross sections as function of axial position................................................ 31

Figure 2.6: IFA-650.3 pellet's cross sections as function of axial position............................................... 32

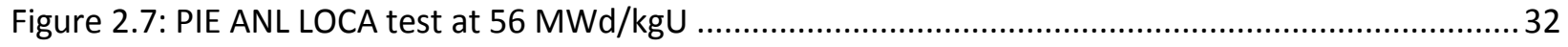

Figure 2.8: Comparison rod inner pressure measurements for IFA-650.3 and IFA-650.4 …....................34

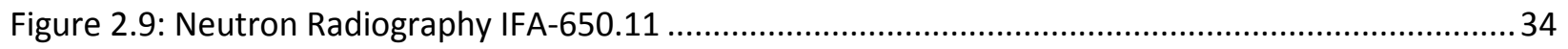

Figure 2.10: Preliminary design based on the conceptual separate effect test .......................................35

Figure 2.11: Details of the experimental set-up and experimental rig ................................................... 37

Figure 2.12: Comparison between commissioning run1 and run 2 .................................................... 38

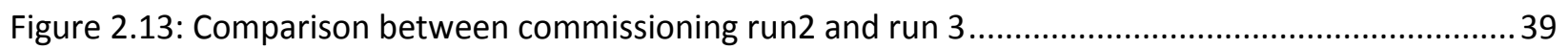

Figure 2.14: Recording of all thermocouple during commissioning Run 2 ........................................... 40

Figure 2.15: Details of the capsule and rod segment design .............................................................. 41

Figure 2.16: ATHLET Model of heat transfer between the capsule and rod segment ............................ 43

Figure 2.17: View factor of concentric infinite cylinders .................................................................... 44

Figure 2.18: Value of emissivity for fresh and irradiated cladding ...................................................... 44

Figure 2.19: Details of ATHLET nodalization of IFA-650 loop ............................................................. 47

Figure 2.20: Details of the ATHLET nodalization of IFA-650 test rig .......................................................48

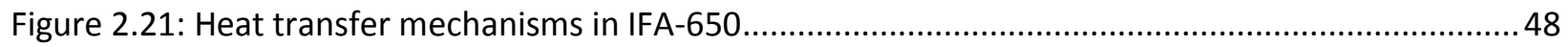

Figure 2.22: TRANSURANUS model for irradiation and LOCA calculations ............................................... 49 


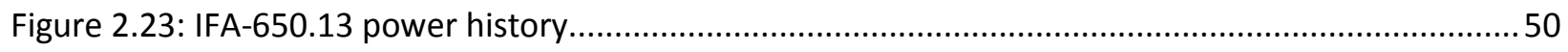

Figure 2.24: MATPRO corrosion correlation for BWR conditions.........................................................51

Figure 2.25: ATHLET IFA-650.13 rig pressure and inlet temperature .....................................................53

Figure 2.26: ATHLET IFA-650.13 temperatures transient ................................................................... 53

Figure 2.27: TRANSURANUS IFA-650.13 end of irradiation radial burnup and fission gases .....................54

Figure 2.28: TRANSURANUS IFA-650.13 LOCA rod internal pressure and cladding deformation .............. 54

Figure 2.29: TRANSURANUS IFA-650.2 LOCA rod internal pressure and cladding deformation .................55

Figure 2.30: TRANSURANUS IFA-650.3 LOCA rod internal pressure and cladding deformation ................55

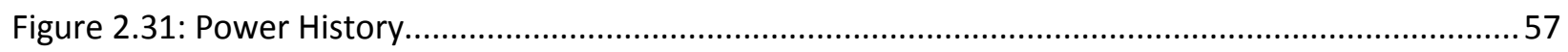

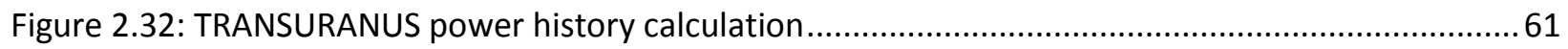

Figure 2.33: TRANSURANUS fuel temperature radial distribution calculation........................................ 61

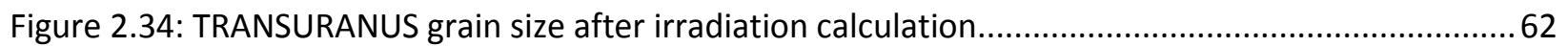

Figure 2.35: TRANSURANUS radial burnup and HBS boundaries calculation ..........................................62

Figure 2.36: TRANSURANUS fission gases distribution after fuel irradiation calculation..........................63

Figure 2.37: Rod1_s1 cladding temperature and inner rod pressure calculations..................................66

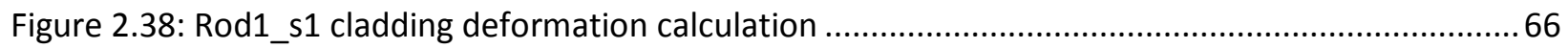

Figure 2.39: Rod2_s1 cladding temperature and inner rod pressure calculations...................................67

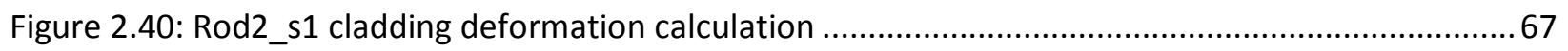

Figure 2.41: TRANSURANUS IFA-650-12 benchmark calculation ........................................................ 71

Figure 2.42: Evolution of rod pressure and cladding temperature IFA-650.12 .................................... 71

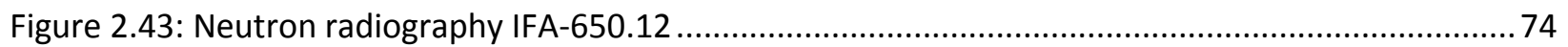

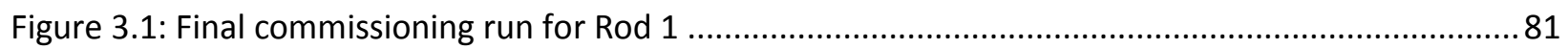

Figure 3.2: Visual inspection of Rod 1 after final commissioning run .................................................. 81

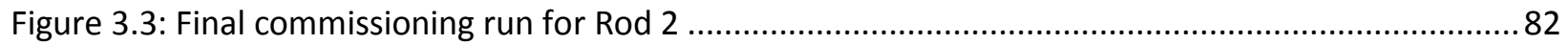

Figure 3.4: Visual inspection of Rod 2 after final commissioning run ................................................... 82

Figure 3.5: Details of the experimental set up and instrumentations ..................................................... 86

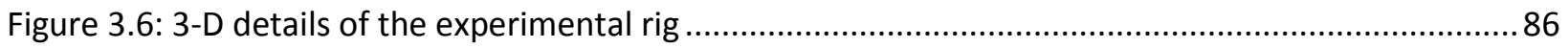

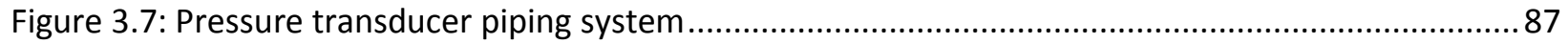

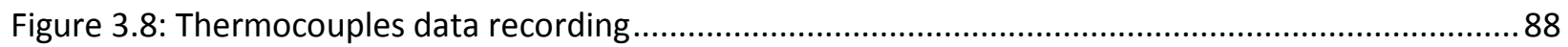

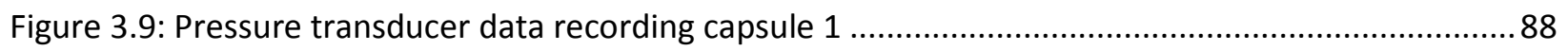

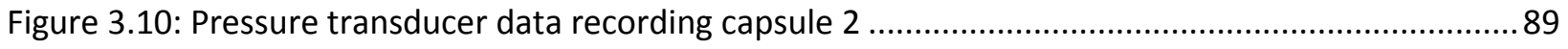

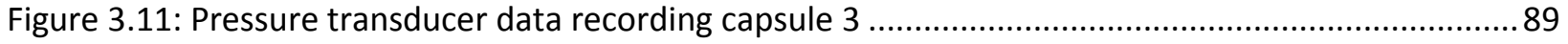


Figure 3.12: Preparatory phase of the experiment execution in the hot cells.....

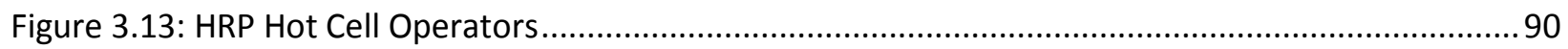

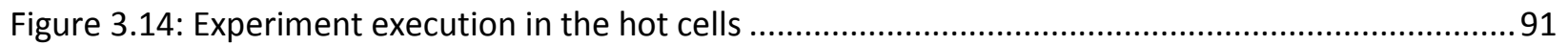

Figure 3.15: Data acquisition system during the experiment ........................................................... 91

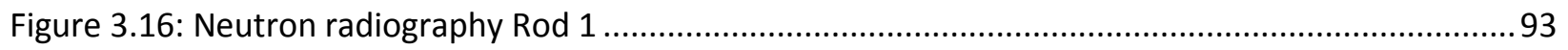

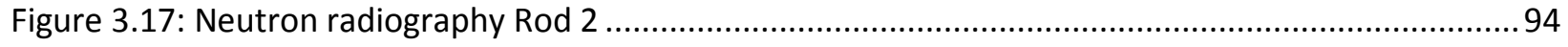

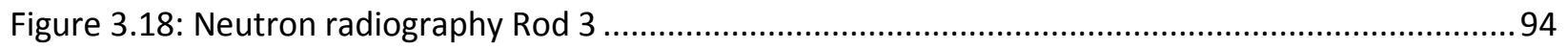

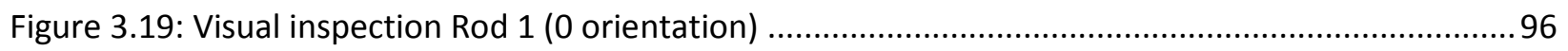

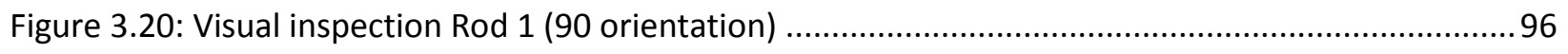

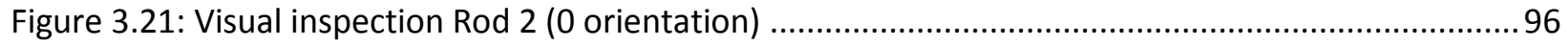

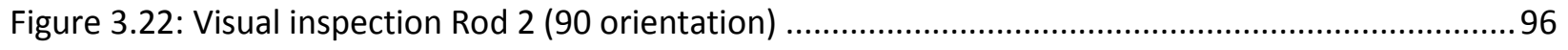

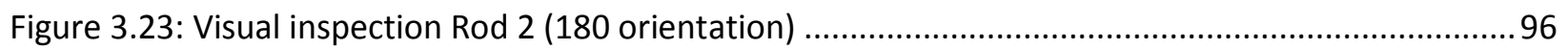

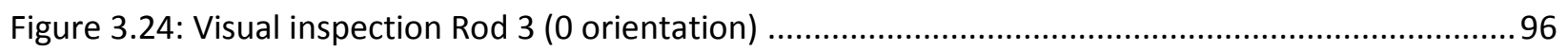

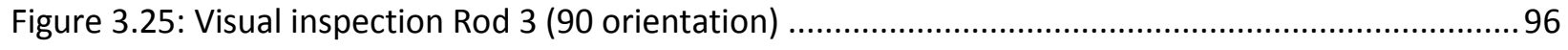

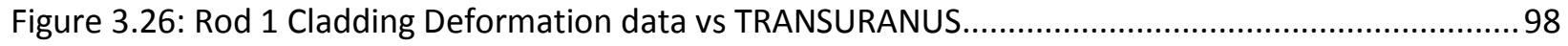

Figure 3.27: Rod 2 Cladding Deformation data vs TRANSURANUS..................................................... 99

Figure 3.28: Rod 3 Cladding Deformation data vs TRANSURANUS.......................................................99

Figure 3.29: Rod 1 inner and outer rod pressure calculated by TRANSURANUS.................................... 100

Figure 3.30: Rod 1 temperature profiles during the transient calculated by ATHLET ............................. 100

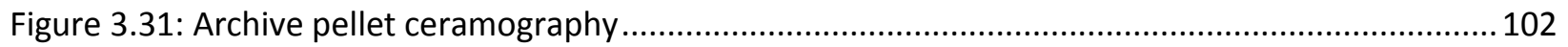

Figure 3.32: Details of ceramography at the center zone of the pellet................................................ 102

Figure 3.33: Details of ceramography at the intermediate zone of the pellet ...................................... 103

Figure 3.34: Details of ceramography at the outer zone of the pellet ................................................ 103

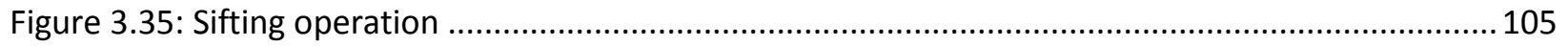

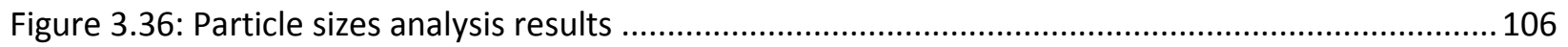

Figure 3.37: Rod 1 and Rod 2 fragment size cumulative distribution.................................................. 107

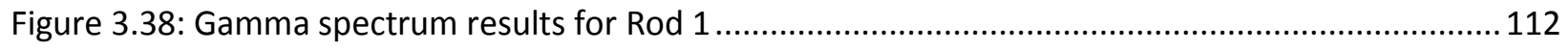

Figure 3.39: Gamma spectrum results for Rod 2 …..................................................................... 113

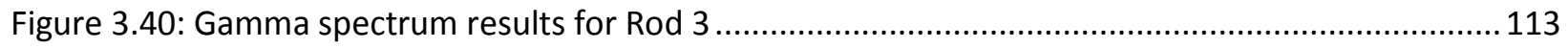

Figure 4.1: Rod 2 fuel fragments during sifting operation................................................................. 117

Figure 4.2: Concrete fragments derived from crushing .................................................................. 118

Figure 4.3: Fuel fragments after LOCA - US NRC Test 193 ............................................................. 119 
Figure 4.4: Fragmentation size distribution for NRC tests at Studsvik

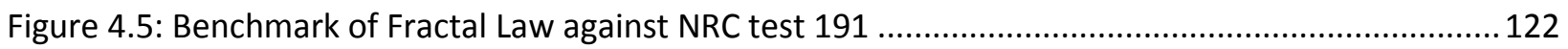

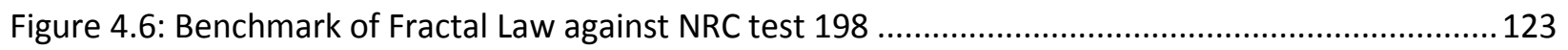

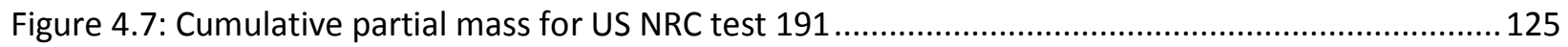

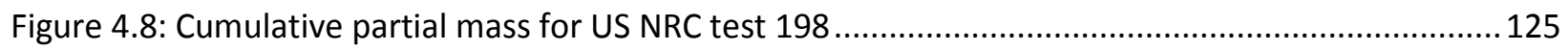

Figure 4.9: Superposition of fractal laws for NRC Studsvik tests ...........................................

Figure 4.10: Neutron Density and Burnup axial Power Distribution for BWR fuel .............................129

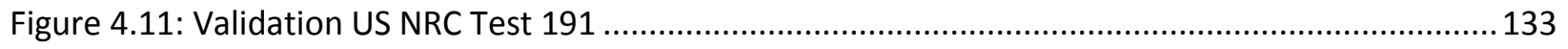

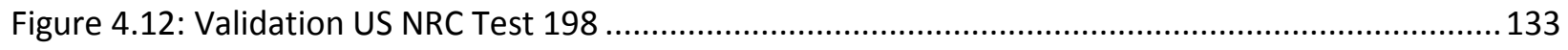

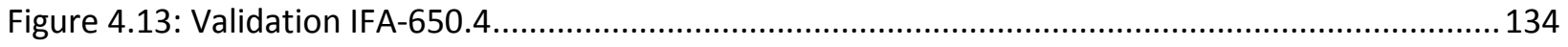

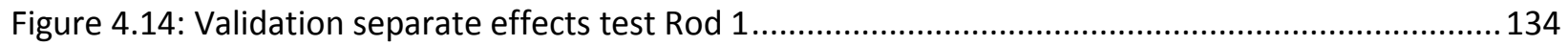

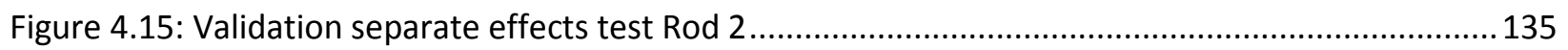

Figure 5.1: Estimation of potential fuel dispersal for Rod 2 .................................................. 140

Figure 5.2: Comparison neutron radiography Rod 2 and visual inspection NRC $198 \ldots \ldots \ldots \ldots \ldots \ldots \ldots \ldots . . . . . .141$

Figure 5.3: Comparison of rod pressure transient Rod 2 and NRC 198.........................................142

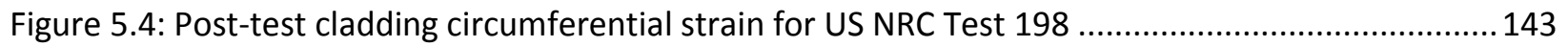

Figure 5.5: Coplanar ballooning in LOCA test at Pacific Northwest Laboratory .................................. 144 


\section{List of Tables}

Table 1.1: Selected outcomes of the literature review of NUREG-2121 .............................................. 12

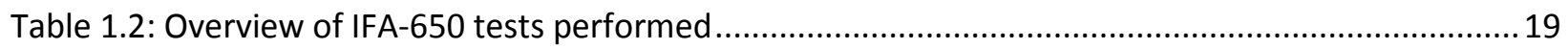

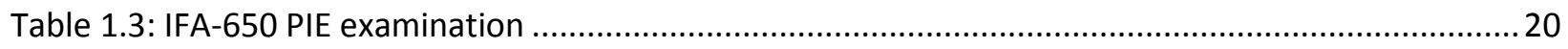

Table 2.1: Comparison of heating up rate of different LOCA experimental program ...............................40

Table 2.2: Properties of the irradiated fuel segment employed in the test ..............................................56

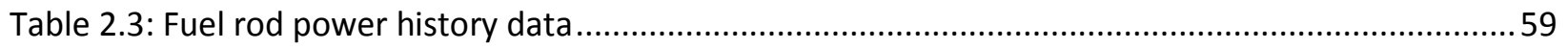

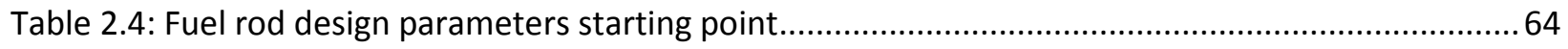

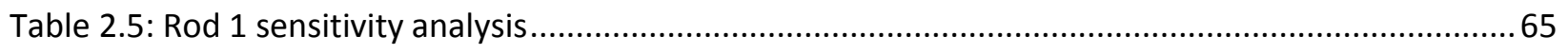

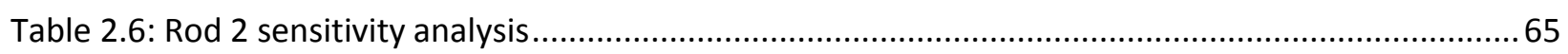

Table 2.7: Selected final fuel rod design parameters ........................................................................6

Table 2.8: Sensitivity on TRANSURANUS grain boundaries saturation approach ................................... 74

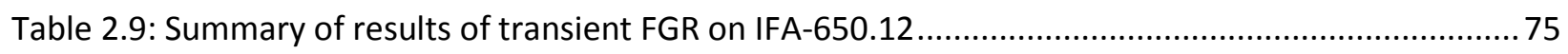

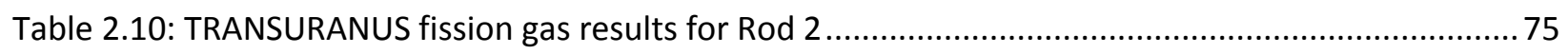

Table 2.11: Prediction of fission gas release for Rod 2 ….................................................................. 76

Table 3.1: Comparison of properties uranium dioxide vs. alumina ...................................................... 80

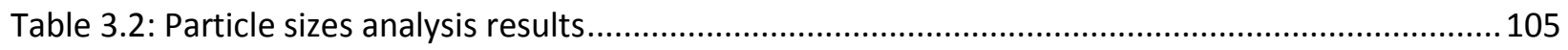

Table 3.3: Parameters for fission gas release calculation .................................................................... 109

Table 3.4: Estimation of fission gas release from the three rods ........................................................ 109

Table 3.5: Application of gamma-ray spectroscopy and isotopes' properties ...................................... 111

Table 3.6: Krypton isotopes production distribution ......................................................................... 112

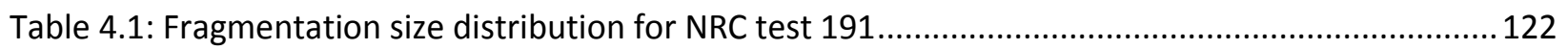

Table 4.2: Fragmentation size distribution for NRC tests 198 .......................................................... 123

Table 4.3: Experiments' data needed for fractal law model validation................................................. 132

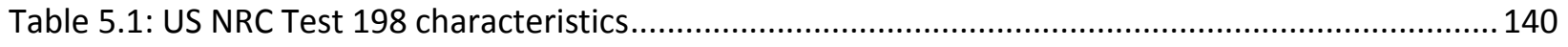




\section{List of Acronyms}

1-D

2-D

3-D

ALHGR

ANL

ATHLET

BCC

BWR

CFR

CSNI

DBA

ECR

ECCS

FGR

GRS

HBS

$\mathrm{HCP}$

HRP

IET

KWU

LB

LHR

LOCA

LPIS

LPRM

LWR

MWd

NEA

NPP

NRC
One Dimensional

Two Dimensional

Three Dimensional

Average Linear Heat Generation Rate

Argonne National Lab

Analysis of Thermal-hydraulics of Leaks and Transient

Body Centered Cubic

Boiling Water Reactor

Code Of Federal Regulations

Committee on the Safety of Nuclear Installations

Design Basis Accident

Equivalent Cladding Reacted

Emergency Core Cooling System

Fission Gas Release

Gesellschaft für Anlagen- und Reaktorsicherheit

High Burnup Structure

Hexagonal Closed Pack

Halden Reactor Project

Integral Effect Test

Kraftwerk Union

Large Break

Linear Heat Rate

Loss Of Coolant Accident

Low Pressure Injection System

Local Power Range Monitor

Light Water Reaction

Megawatt day

Nuclear Energy Agency

Nuclear Power Plant

Nuclear Regulatory Commission 
OECD

PCT

PDD

PIE

P\&ID

PWR

$\mathrm{RCT}$

RPS

SCRAM

SPND

SET

US
Organisation for Economic Co-operation and Development

Peak Cladding Temperature

Power Density Detector

Post Irradiation Examination

Piping and Instrumentation Diagram

Pressurized Water Reactor

Rig Compression Test

Reactor Protection System

Safety Control Rod Axe Man

Self-Powered Neutron Detectors

Separate Effect Test

United States 
chapter 1

\section{Introduction}

\section{Contents}

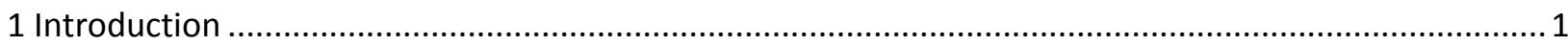

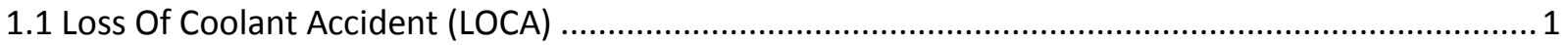

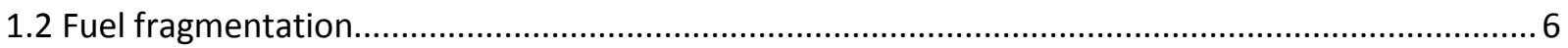

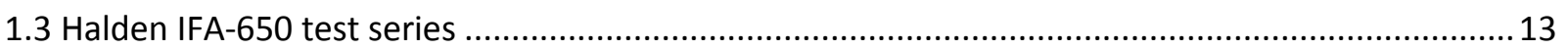

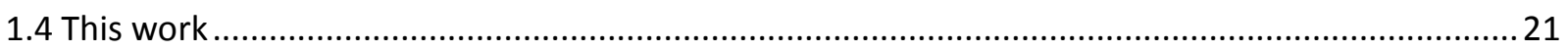

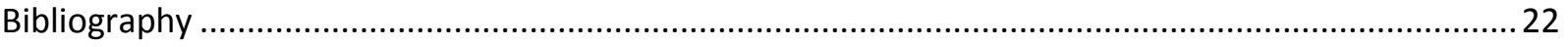

\subsection{Loss Of Coolant Accident (LOCA)}

Since the beginning of civil nuclear technology, the Loss Of Coolant Accident (LOCA) for Light Water Reactors (LWRs) has been under special focus of international regulatory bodies, fuel design vendors and utilities because it was one of the Design Basis Accidents (DBAs) of most of reactor concepts [1]. For design basis accidents, a fundamental acceptance criterion is that there should be no or very limited radiological consequences to the environment [2]. In order to fulfil this criterion, derivative acceptance criteria have been formulated for the safety systems of LWRs. The safety criteria vary from country to country. However, most of the criteria are taken from the criteria established by the United States (US) Nuclear Regulatory Commission (US NRC). In the US, the LOCA acceptance criteria were introduced in 1973 in the Title 10 of the Code of Federal Regulations (10 CFR) 50.46 (b) "Acceptance criteria for emergency core cooling system for light-water nuclear power reactors" [3].

Depending on the LWR technology, Pressurized Water Reactor (PWR) or Boiling Water Reactor (BWR) and on the specific design features, the DBA LOCA involves the break of one of the large coolant pipes of the reactor primary circulation system. Emergency Core Cooling System (ECCS) are designed to 
guarantee the preservation of the primary fuel safety barriers through injection of cooling water so that the fuel is cooled efficiently during all phases of the accident. In consequence the fuel maintains its coolable geometry and structural integrity.

The requirement of coolable geometry and structural integrity has turned out to be a very complex issue due to the particular properties of the zirconium alloy used as cladding for the fuel. There are five cladding embrittlement mechanisms:

- Beta layer thinning;

- Beta layer embrittlement by oxygen;

- Localized hydrogen-induced embrittlement in the ballooned area;

- Hydrogen-enhanced beta layer embrittlement by oxygen;

- Hydrogen-induced embrittlement from breakaway oxidation.

During the event, the cladding heats up to temperature above $1000^{\circ} \mathrm{C}$. When the temperature reaches about $800{ }^{\circ} \mathrm{C}$, zirconium starts to transform from alpha to beta phase. Simultaneously the oxygen can diffuse from the $\mathrm{ZrO}_{2}$ to the bulk cladding with increased hydrogen levels from the zirconium oxidation increasing the oxygen solubility. Wherever the solubility limit of oxygen in the beta phase is exceeded, thee excess oxygen stabilizes the alpha phase. The oxygen stabilized alpha phase grows next to the $\mathrm{ZrO}_{2}$ layer at the expense of the bulk cladding being in the beta phase at high temperatures. Only this part of the cladding, called prior-beta phase after quench, can be credited with any load bearing capability. The oxygen solubility limit in the alpha and beta phases is temperature dependent (e.g. the beta phase can hold only about 0.7 wt\% oxygen) [4]. Therefore, there must be a limit on the oxygen-stabilised alpha phase, since the load bearing prior beta layer may be too thin to ensure structural integrity of the fuel during the quench phase of the LOCA.

In the last decades many experiments and further investigations have been performed concerning LOCA with focus on thermal-hydraulics [5] and fuel performance [6]. These experimental programs include inpile fuel tests, out-of-pile fuel tests, Separate Effect Tests (SET) and Integral Effect Tests (IET). The goals of these investigations were the understandings of different thermal-hydraulics and fuel performance phenomena and the development of purely analytical, semi-empirical and empirical models needed for the modelling of a LOCA in a commercial Nuclear Power Plant (NPP) with the ultimate goal to create and verify a robust ensemble of safety criteria.

Figure 1.1 shows the typical behaviour of the cladding temperature of a hot rod during a Large Break LOCA (LB LOCA) in a typical PWR. The figure shows selected, relevant fuel performance and thermalhydraulics phenomena, in addition to the main licensing requirements. 


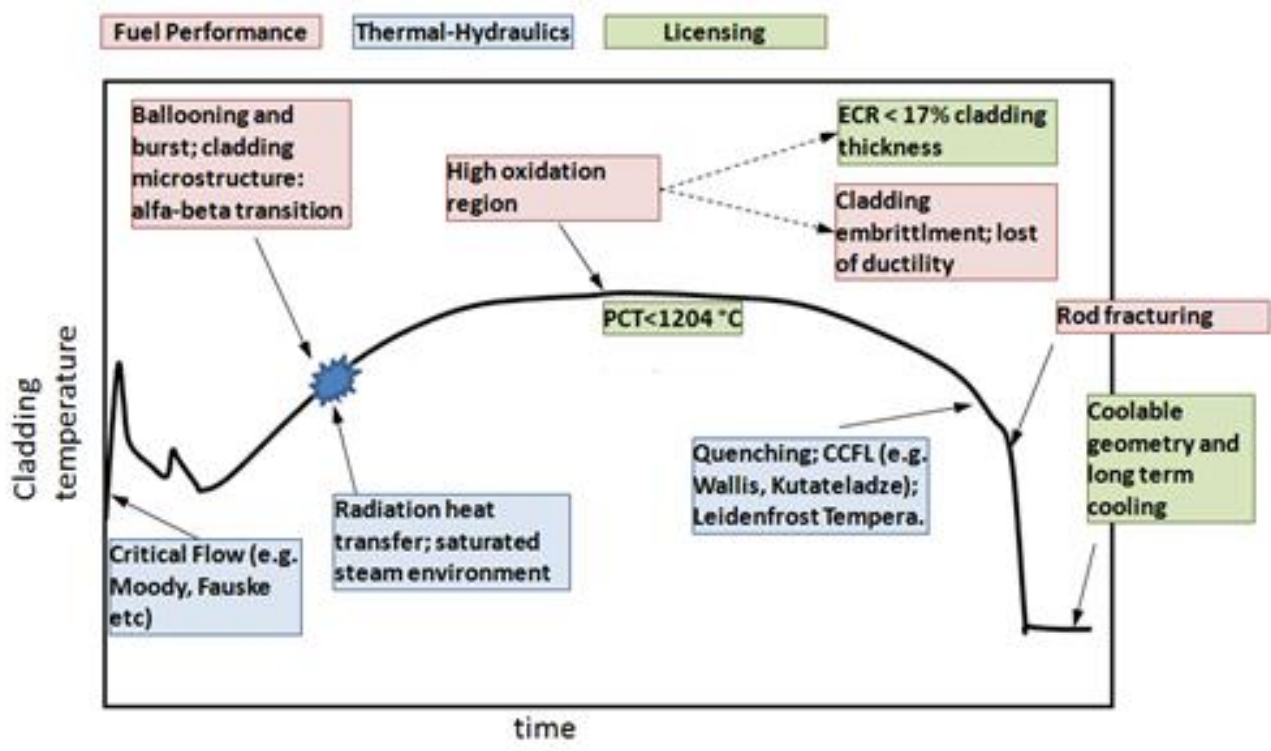

Figure 1.1: Prototypical LOCA transient for a typical PWR PCT: Peak Cladding Temperature; ECR: Equivalent Cladding Reacted.

During a LB LOCA, the reduction of fluid inventory in the core and the flashing due to the fast depressurization quickly reduces the reactor power. Additionally, due to the activation of the Reactor Protection System (RPS), reactor SCRAM is activated within few seconds. For the sake of being conservative with respect of stored energies in an LB LOCA event, no credit for rod insertion is taken in the analysis. The energy that needs to be removed from the core corresponds to the stored energy generated before the event and from the decay heat production from fission products and from actinides such as $\mathrm{U}_{239}$ and $\mathrm{Np}_{239}$. Examples of decay heat power from fission products are presented through tables and analytical representations [7].

When a pipe break occurs in the primary system of a NPP, high pressure primary fluid is discharged into the containment environment. Since the pressure of the containment is smaller than the critical pressure, critical flow occurs. Broadly applied critical flow correlations are summarized by Todreas and Kazimi [8]. After an initial phase in which the heat transfer from the fuel is enhanced due to flashing, with the diminished cooling and the release of stored energy in the fuel, the cladding heats up.

Since the reactor coolant pressure drops below the internal fuel gas pressure given by the initial fill-in gas plus the fission gas released during irradiation, the fuel cladding swells (ballooning) and, under some conditions, burst occurs. Models have been developed to predict the cladding ballooning and rupture conditions [9].

The ECCS is activated after the break. Injection from the cold and/or hot leg accumulators is activated when the pressure of the accumulator is above the rapidly decreasing primary system pressure. However, most of the flow of the cold legs accumulators is swept around the downcomer, into the broken-loop cold leg and out of the break. If the reactor design is equipped with hot legs accumulators, the cooling of the colder area of the core starts quickly. 
As the blowdown progresses, an increased amount of the accumulator-injected coolant begins to enter in the lower plenum. Conservatively assuming a loss-of-site power and applying single failure assumption for the ECCS, the injection time from the Low Pressure Injection System (LPIS) is a function of the emergency generator (diesel) starting time and the time needed from the diesel to bring LPIS pumps to nominal speed. After the injection starts, the lower plenum can be filled and the core begins to refill. The hot fuel rods continue to heat up until the quench front begins to move upward through the core. As the quench front continues to advance, the fuel rod upper elevations are cooled by a dispersed non-equilibrium two-phase mixture of superheated steam and entrained droplets. Eventually, there is sufficient cooling in advance of the quench front to terminate the increase in cladding temperature and the Peak Cladding Temperature (PCT) is reached. Figure 1.1 is purely a prototypical cladding transient behaviour during LOCA for a LWR and the maximum PCT can be reached at different transient phases depending on the reactor technology and design.

Consequently, the LOCA transient involves an excursion in temperature for parts of the reactor core in which the reaction of the Zircaloy fuel rods with the steam environment may become appreciable (high temperature steam oxidation). When zirconium is exposed to steam, the zirconium is oxidized at a more rapid rate than during normal operation (corrosion), therefore at temperature above $1000^{\circ} \mathrm{C}$ the cladding surface oxidizes rapidly. The oxidation rate is conservatively described by Baker-Just correlation [10].

When finally the LPSI refloods the core, temperature will begin to decrease and below $800^{\circ} \mathrm{C}$ the cladding metal will return to alpha phase. When the cladding temperature drops below the minimum film boiling temperature in the range of $600-800^{\circ} \mathrm{C}$, the cladding quenches and the temperature falls quickly to the average water temperature. At this lower temperature, oxygen diffusion is effectively stopped and the high-temperature oxygen distribution is frozen into the cladding. Zircaloy metal containing more than about $0.6 \mathrm{wt} \%$ oxygen is found to be brittle. Therefore, only the low-oxygen material, which was still in the beta phase when it was at high temperature, is ductile.

The LOCA acceptance criteria as included in Figure 1.1 were introduced in 1973 in the Title 10 of the Code of Federal Regulations (10 CFR) 50.46 (b) "Acceptance criteria for emergency core cooling system for light-water nuclear power reactors":

1 Peak cladding temperature: The calculated maximum fuel element cladding temperature shall not exceed $2200^{\circ} \mathrm{F}\left[1204^{\circ} \mathrm{C}\right]$.

2 Maximum cladding oxidation: The calculated total oxidation of the cladding shall nowhere exceed 0.17 times the total cladding thickness before oxidation. As used in this subparagraph total oxidation means the total thickness of cladding metal that would be locally converted to oxide if all the oxygen absorbed by and reacted with the cladding locally were converted to stoichiometric zirconium dioxide. If cladding rupture is calculated to occur, the inside surfaces of the cladding shall be included in the oxidation, beginning at the calculated time of rupture. Cladding thickness before oxidation means the radial distance from inside to outside the cladding, after any calculated rupture or swelling has occurred but before significant oxidation. Where the calculated conditions of transient pressure and temperature lead to a prediction of cladding swelling, with or without 
cladding rupture, the unoxidized cladding thickness shall be defined as the cladding cross sectional area, taken at a horizontal plane at the elevation of the rupture, if it occurs, or the elevation of the highest cladding temperature if no rupture is calculated to occur, divided by the average circumference at that elevation. For ruptured cladding the circumference does not include the rupture opening.

3 Maximum hydrogen generation: The calculated total amount of hydrogen generated from the chemical reaction of the cladding with water or steam shall not exceed 0.01 times the hypothetical amount that would be generated if all of the metal in the cladding cylinders surrounding the fuel, excluding the cladding surrounding the plenum volume, were to react.

4 Coolable geometry: Calculated changes in core geometry shall be such that the core remains amenable to cooling.

5 Long-term cooling: After any calculated successful initial operation of the ECCS, the calculated core temperature shall be maintained at an acceptably low value and decay heat shall be removed for the extended period of time required by the long-lived radioactivity remaining in the core.

In Figure 1.1 ECR stands for Equivalent Cladding Reacted and correspond to criteria number 2. In particular criteria 1 and 2 are intended to assure that the cladding retains some ductility during and following the quench, because only the low-oxygen material, which was in the beta phase when it was at high temperature, is ductile. Hache and Chung provided a very extensive description of the history of LOCA embrittlement criteria [11]. Criterion number 3 is not included in the Figure because its purpose is to limit the amount of hydrogen generation during the accident so to avoid an explosive concentration of hydrogen gas.

Other countries such as Germany and Finland included a criterion to comply with the boundary conditions of radiological analysis [12] limiting the fraction of burst rods in the core (failure rate) to a value less than $10 \%$.

The NRC criteria implemented in 1973 were based largely on Ring-Compression Tests (RCTs) to assess the oxidation and temperature limits at which embrittlement occurs and the data available at the time were largely from as-fabricated and low-burnup cladding materials.

In the 90s, internationally, several research programs started to investigate the effects of high burnup operation on LOCAs and other accidents. A broad research program started in 1997 at the Argonne National Laboratory (ANL) in which the effects of high burnup operation on cladding embrittlement following a LOCA were assessed.

ANL collected as-fabricated cladding alloys and high burnup fuel rods for testing by many industry organizations. Pre-hydrided cladding was prepared from as-fabricated Zircaloy-4 to study the effects of hydrogen over a wide range of oxidation conditions and cooling scenarios. The primary testing method consisted of oxidation in steam at high temperatures and cooling with or without quench at $800^{\circ} \mathrm{C}$, followed by ring-compression ductility testing at room temperature and $135^{\circ} \mathrm{C}$ after cool down.

The main finding concerned the cladding embrittlement criteria because it was proven that hydrogen, which enters the outer cladding surface during normal lifetime operation, reduces cladding ductility. 
Figure 1.2 summarizes the results of [13] with the reduction of the ductility threshold for different typical cladding types (Zircaloy-4, ZIRLO and M5). Based on the outcomes of [13] the US NRC published a draft of a regulatory guide [14] to establish analytical limits for Zirconium-based alloy cladding.

Another finding of the ANL research was that for oxidation times bigger than 1000 seconds, two temperature ranges around circa $750^{\circ} \mathrm{C}$ and $950^{\circ} \mathrm{C}$ exist which tend to support the transformation of the compact tetragonal oxide into the porous monoclinic oxide. The phase transformation is accompanied by an increased oxidation rate („,breakaway“) compared to normal high-temperature oxidation kinetics and high hydrogen pickup. This phenomenon is referred to as "breakaway oxidation". For LB LOCA this phenomenon may be important and depends upon the reactor technologies and their ability to quickly quench the reactor core. As example, German PWRs quench at about $125 \mathrm{~s}$ [15] and consequently there are no safety issues for current fuel average discharge burnup and cladding materials.

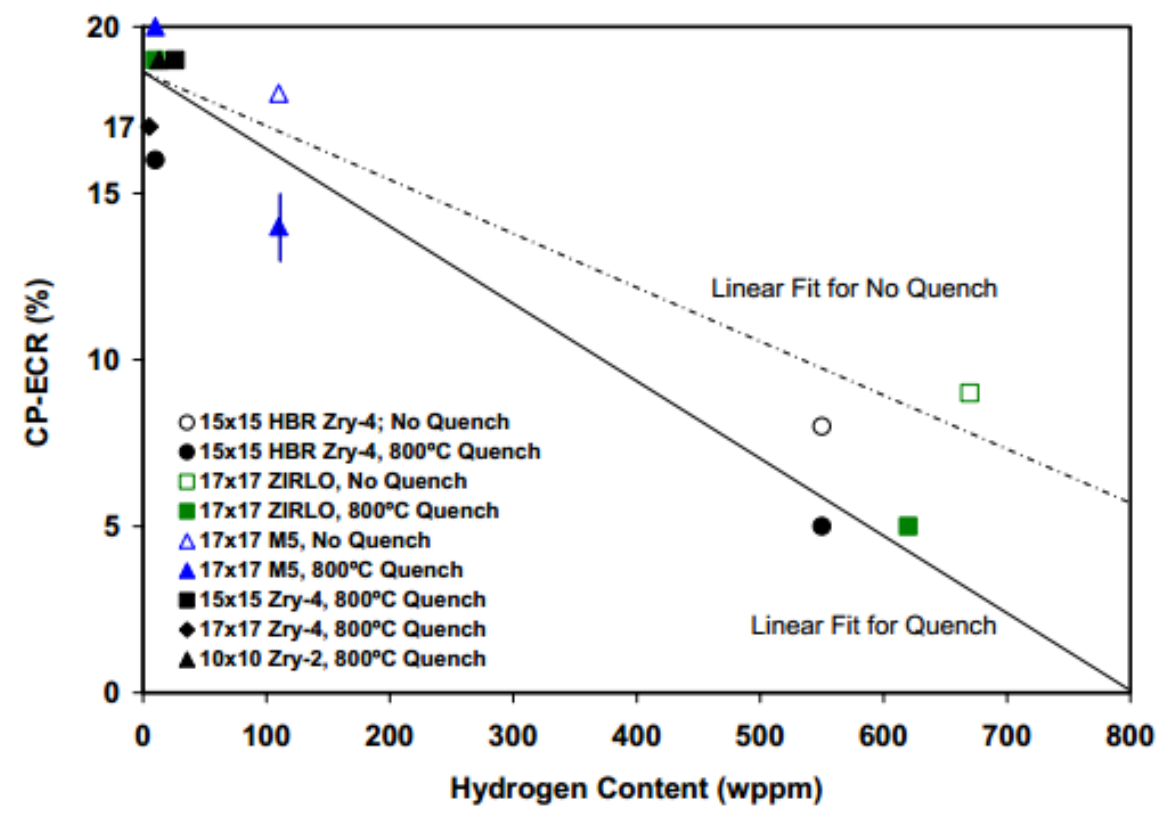

Figure 1.2: Reduction of ductility threshold as function of cladding hydrogen content The Figure use Cathcart-Pawel (CP) weight-gain correlation to calculate CP-ECR

Figure from Reference [13]

\subsection{Fuel fragmentation}

As part of the international efforts to re-examine the LOCA safety criteria and to verify their validity with the move to high burnup and new cladding materials, the Organisation for Economic Co-operation and Development (OECD) Halden Reactor Project (HRP) implemented a LOCA test series IFA-650 and the safety significance of the Halden IFA-650 test results was firstly addressed in 2010 in a report published by the Committee on the Safety of Nuclear Installations (CSNI) of the Nuclear Energy Agency (NEA) [16]. 
LOCA tests at the Halden are integral in-pile single rod tests and the test series were designed to address various LOCA issues. High burnup fuel rods irradiated in commercial reactors have been used. The test conditions are planned to meet the following primary objectives $[17,18]$ :

- Maximize the ballooning size to promote fuel relocation and to evaluate its possible effect on the cladding temperature and oxidation: Fuel fragmentation and potential relocation are not novel phenomena. LOCA tests executed before 1984 showed already fuel fragmentation. The US NRC published a generic safety issue GSI-92 "Fuel Crumbling during LOCA" [19] in which the safety significance of the observed fuel crumbling was made. The main item of concern was the relocation of the fuel fragments in the ballooning region thus increasing locally the stored energy and energy generated from radioactive decay. Since the existing ECCS performance analysis codes did not account for fuel relocation in the ballooning region, this lack of inclusion was considered a non-conservatism that needed to be addressed. The generic issue received a low prioritization because it was concluded that temperature increases due to relocation of crumbled fuel would be smaller than those due to other processes that are treated conservatively in Appendix $\mathrm{K}$ to part 10 CFR 50 that prescribes the required and acceptable features of ECCS evaluation models.

- Investigate the extent (if any) of "secondary transient hydriding" on the inner side of the cladding around the burst region: the secondary hydriding transient is a phenomenon investigated also by ANL in [13]. Due to steam leakage through the burst opening, oxidation within the balloon region is expected to be two-sided. Such oxidation results in hydrogen release to the near-stagnant steam within the test sample. Unlike the outer surface exposed to flowing steam, a large fraction of the released hydrogen is trapped inside the sample and available for pickup by the inner-metal surface in and near the balloon region.

- Measure the release of iodine and caesium from failed fuel in LOCAs: this objective was added only in 2009 and applied to the tests executed since then.

The fourth test of the series, IFA-650.4, caused particular attention in the international nuclear community. The fuel in this experiment had a high burnup, $92 \mathrm{MWd} / \mathrm{kgU}$. The rod ballooned as intended and the burst caused substantial fuel relocation, and PIE revealed considerable fuel fragmentation. A similar result was obtained with a later test, IFA-650.9, likewise using high burnup fuel.

Figure 1.3 shows the status of the fuel after the test IFA-650.4. On the left side of the Figure, the fuel relocation was verified by gamma scanning with a big portion of the top fuel stack missing, dropping to the ballooned region at half height of the rod and partly at the bottom of the flask containing the experimental rig. On the right, fuel fragmentation was verified with a fuel cross section cut. 


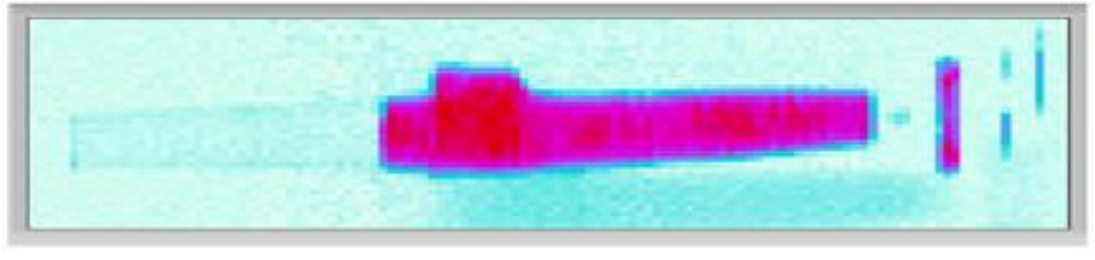

(a)

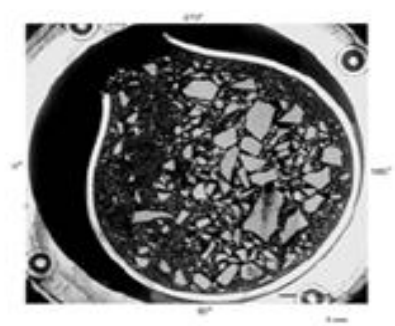

(b)

Figure 1.3: IFA-650.4 fuel rod status after the test

a) Neutron Radiography [16]; b) Destructive test fuel cross section analysis [16]

The Halden tests have identified fuel behavior not previously seen, in particular, concerning the strong fuel fragmentation and dispersal. The results were considered to some extent produced and amplified by conditions and features deliberately introduced into the test series. The most important were:

- Fuel: The fuel had a burnup of more than $90 \mathrm{MWd} / \mathrm{kgU}$. Many phenomena are known that are progressively affected by exposure, and the burnup of the Halden LOCA test segments was clearly above today's LWR local discharge burnup.

- Outer geometry: The IFA-650 is a single rod test surrounded by an electrical heater and there is no presence of an azimuthal temperature gradient. Experimental data show that a uniform azimuthal temperature in the cladding promotes larger ballooning [20]. The potential contact between adjacent rods may enhance the azimuthal gradient and therefore further limiting ballooning.

- Rod design: The axial length is about $50 \mathrm{~cm}$ and there is a considerable larger free volume to fuel ratio with respect to a commercial reactor. The latter combined with the rod pressure provides for relatively more gas to drive the ballooning, fuel relocation and dispersal reflecting that large ballooning was a major test objective.

- The cladding material used was Duplex type and the fuel rod showed very small oxidation. The cladding material is believed to be particularly ductile for a very high burnup fuel so to produce a bigger ballooning.

Consequently, the HRP planned further tests with fuel burnup closer to the current LWR local discharge burnup of approximately 65-70 $\mathrm{MWd} / \mathrm{kgU}$ and corrective actions were taken also concerning the dimension of the upper free volume.

The US NRC has performed additional LOCA tests at the Studsvik laboratory in Sweden with high burnup fuel rods to investigate the mechanical properties of ballooned and ruptured cladding [21]. Even though the focus of the experiment was on the cladding behavior, during the test significant fuel fragmentation, mobility and release were recorded. The fuel rods had a burnup between 55 and about $75 \mathrm{MWd} / \mathrm{kgU}$. The test was an out-of-pile test with no nuclear heating and the external heating was provided by a clam-shell radiant furnace. In each experiment, single rod segments of pressurized, irradiated and fuelled cladding, approximately $300 \mathrm{~mm}$ in length, were ramped in steam from $300^{\circ} \mathrm{C}$ to a target 
temperature of about $1200^{\circ} \mathrm{C}$ at a rate of $5^{\circ} \mathrm{C} / \mathrm{sec}$. Internal pressures were consistent with end of life rod internal pressure and hold times were selected to achieve various oxidation levels (ECR).

Figure 1.4 and Figure 1.5 show the rupture opening and the fragments collected for test 193 after the transient (BU: $77 \mathrm{MWd} / \mathrm{kgU}$; PCT: $1160^{\circ} \mathrm{C}$; fill in pressure: $82 \mathrm{bar}$ ) and 198 (BU: $55 \mathrm{MWd} / \mathrm{kgU}$; PCT: $1160^{\circ} \mathrm{C}$; fill in pressure: $82 \mathrm{bar}$ ).

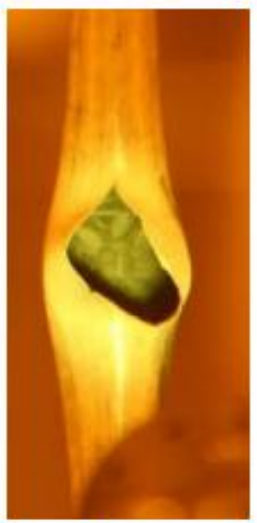

(a)

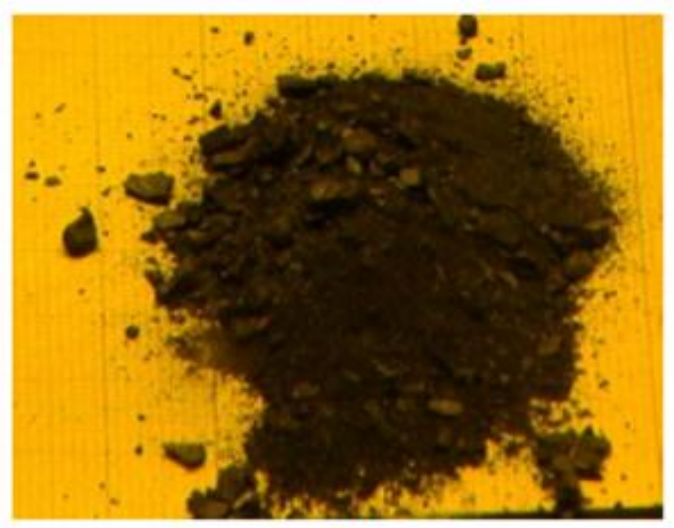

(b)

Figure 1.4: NRC Studsvik Test number $193-71 \mathrm{MWd} / \mathrm{kgU}$

a) Cladding opening after the test [21]; b) Fuel particles collected after the test [21]

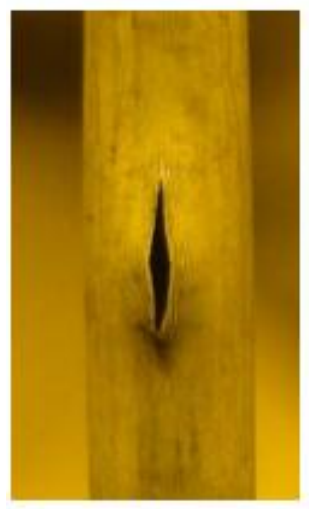

(a)

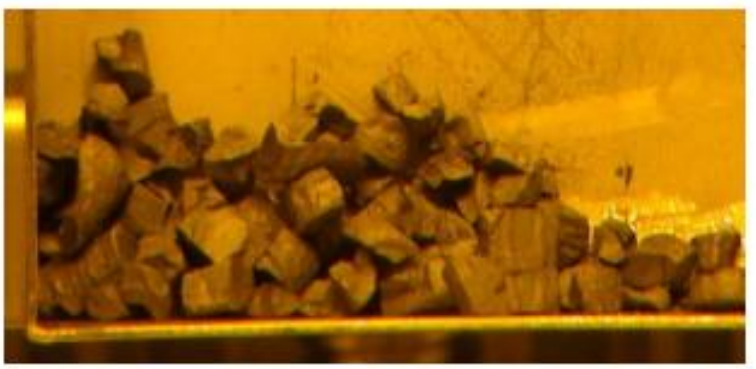

(b)

Figure 1.5: NRC Studsvik Test number $198-55 \mathrm{MWd} / \mathrm{kgU}$

a) Cladding opening after the test [21]; b) Fuel particles collected after the test [21]

The main observation that can be drawn looking at the two figures above is that the rupture opening size is much bigger at higher burnup despite a similar heating up rate, target PCT and rod internal pressure. In addition, the particle size distribution is also extremely different with fuel rod particles of different sizes up to powder for test 193 and mostly big fragments of the order of magnitude of several millimeters for test 198.

The topic of fuel fragmentation and dispersal regained attention in the international community after the NRC LOCA tests results, in particular because the burnup was very similar to the LWR discharge 
burnup. The limit LWR discharge burnup depends on different countries and it can be given as a direct limit or it may be given by indirect criteria in which parameters involved in the process are influenced by the burnup. For instance in the US, the RIL-0801 [22] concluded that a burnup limit of $62 \mathrm{MWd} / \mathrm{kgU}$ (average for the peak rod) was probably low enough to prevent significant fuel loss during LOCA.

In light of the results from the Studsvik test, the US NRC published a comprehensive review of past research programs about observations related to the phenomena of fuel fragmentation, relocation and dispersal [23]. Particular emphasis was given to the fuel release and therefore to the relation between fuel rod opening and fuel particle size. The possibility that fuel particles could escape from the fuel rod and enter the reactor coolant system was never systematically analyzed. The report points out postulated consequences of larger fuel dispersal that include: fuel coolant interaction, radiological effects, flow blockage and hydraulic effects and mechanical interference with pumps and valves necessary to ECCS to perform.

The report includes the review of over 90 LOCA test results performed in eight different programs over the last 35 years. The eight programs are the following:

1. Power Burst Facility, Idaho National Engineering Laboratory (United States of America)

2. FR-2, Karlsruhe Institute of Technology (Germany)

3. National Research Universal, Pacific Northwest Laboratory (Canada)

4. PHEBUS-LOCA, Institut de Radioprotection et de Sûreté Nucléaire (France)

5. FLASH Tests, Commissariat à l'Energie Atomique (France)

6. Argonne National Laboratory (ANL) LOCA Test Program (United States of America)

7. Halden Boiling Water Reactor LOCA IFA-650 Test Series (Norway)

8. Studsvik LOCA Test Program (Sweden)

In Table 1.1 there is a summary of the characteristics and test results of the 8 programs with key figures and information extracted from [23]. Observing the selected pictures from Table 1.1, fuel fragmentation can also occur for very small burnup despite the different experimental facilities, purpose of the investigation and boundary conditions. The NRC staff concluded that the fuel fragment size distribution is not uniform and the average fragment size decreases with increasing burnup and additional considerations were derived concerning fuel axial relocation and dispersal:

- Axial relocation occurs in the presence of appreciable cladding diametral strain accompanied by appreciable fragmentation.

- Grid spacers may hinder rod ballooning, potentially acting as choke points for fuel relocation.

- The rod fill pressure has an important effect on rupture, the characteristics of the balloon, and rupture opening sizes and shapes.

- The amount of fuel that is dispersed can vary widely, from a puff of dust to large amounts of fragmented and pulverized fuel. 


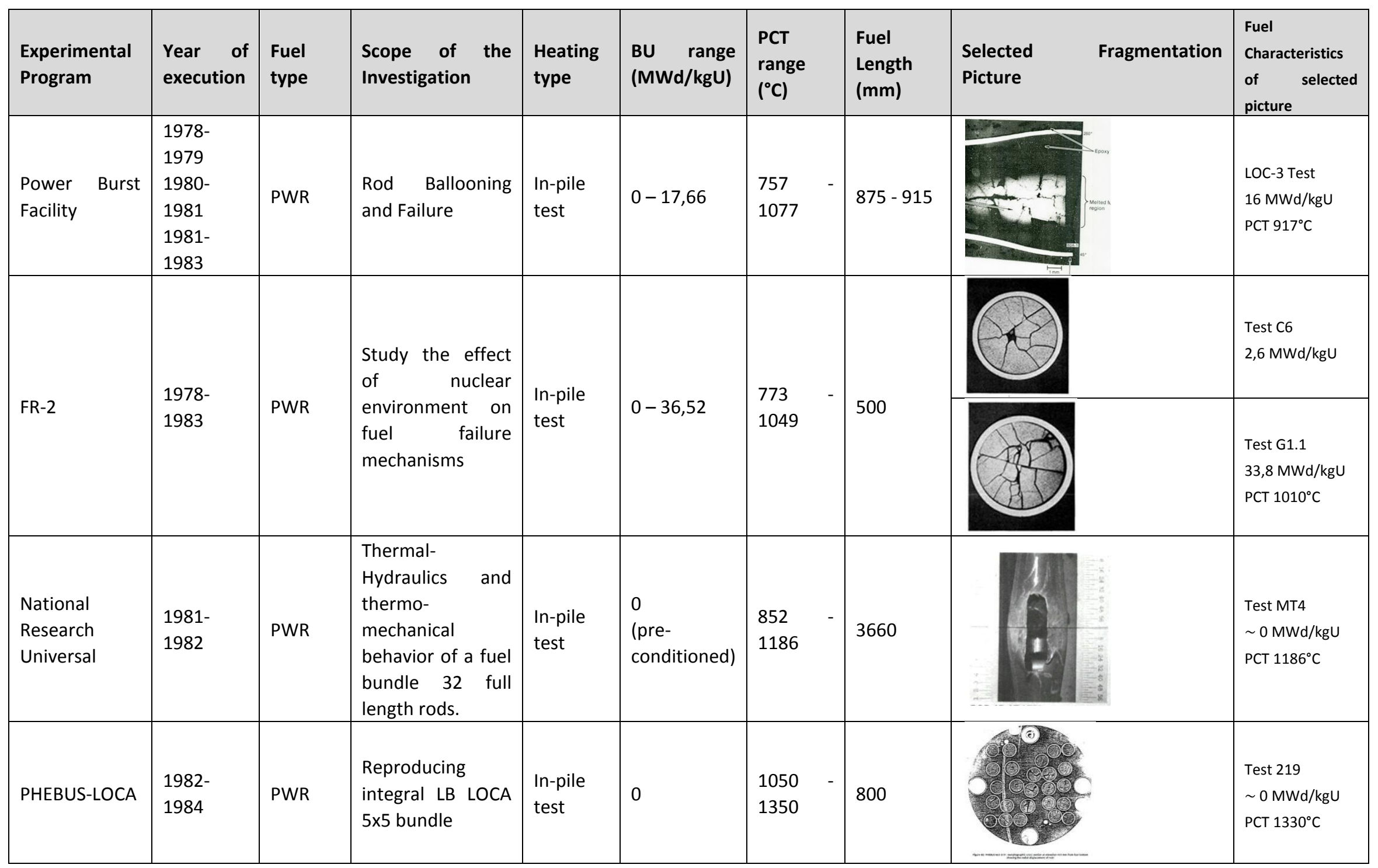




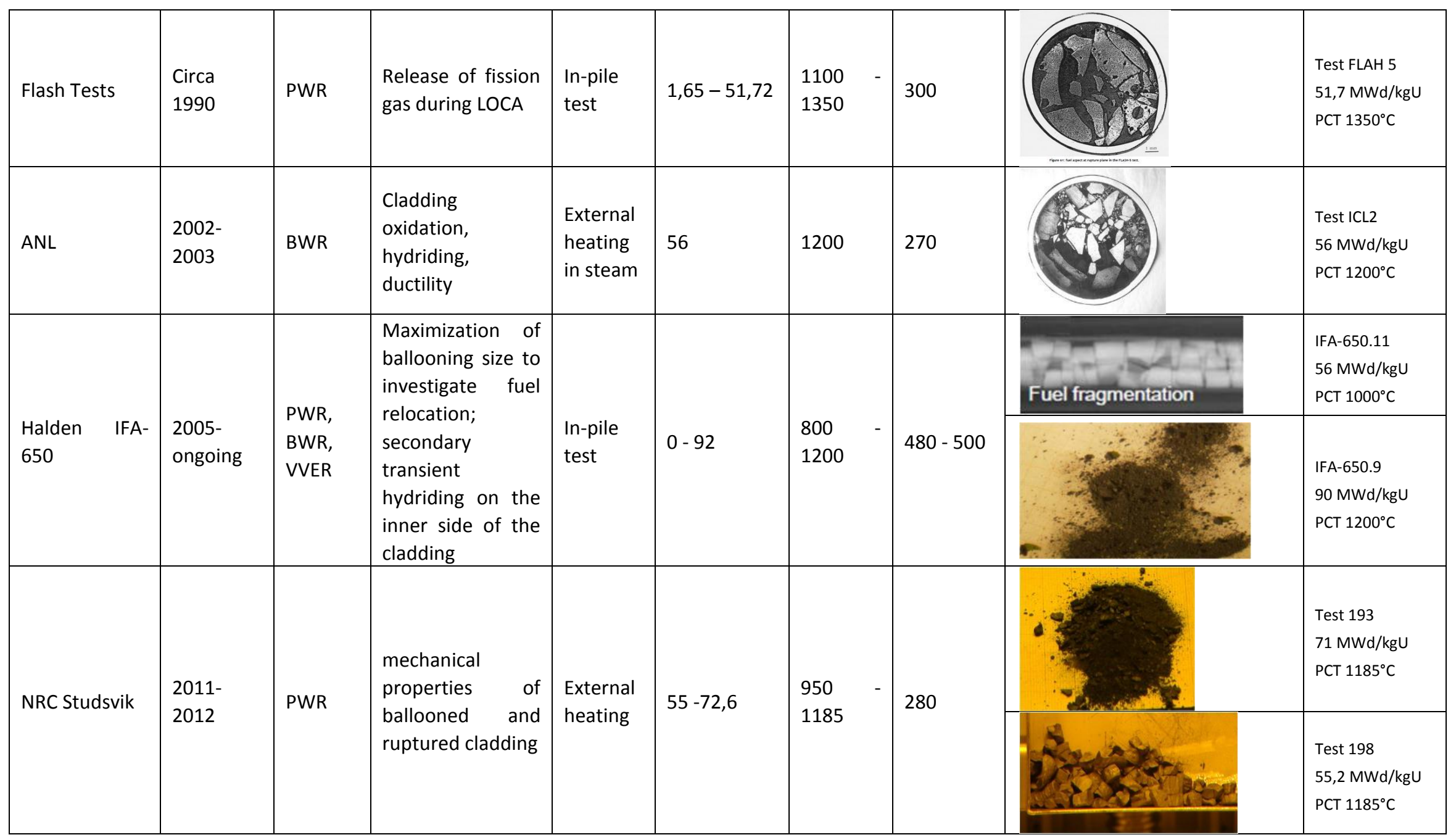

Table 1.1: Selected outcomes of the literature review of NUREG-2121

Information and figures selected from [23] 


\subsection{Halden IFA-650 test series}

The Halden LOCA test IFA-650 provides an enormous database to study fuel performance during LOCA. The IFA-650 test series have been extensively used during this study for observations related to fuel fragmentation and to validate a LOCA methodology calculation with ATHLET [24] and TRANSURANUS [25]. A good overview of the LOCA test series IFA-650 is provided in [26].

The experiments are performed in-core inside the Halden Boiling Water Reactor. Heating is provided internally by simulating the decay heat through a low level of nuclear power. The rig structure and instrumentation are shown in Figure 1.6.

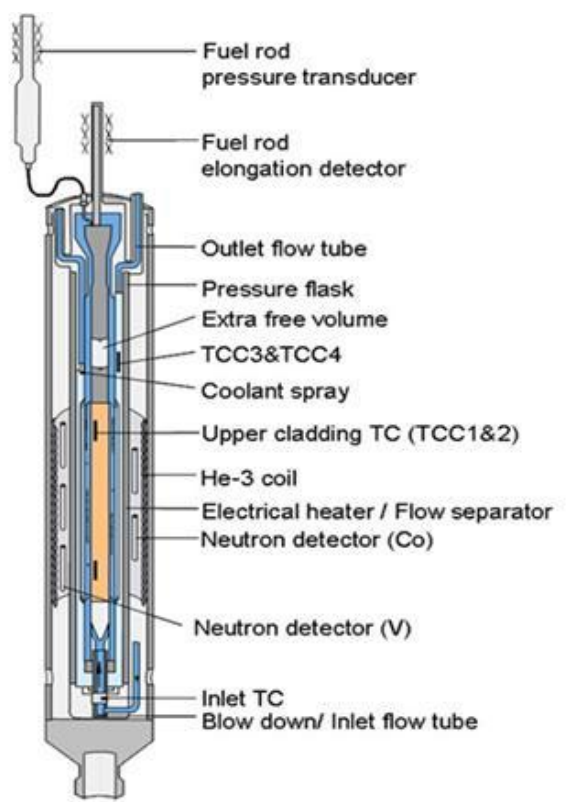

(a)

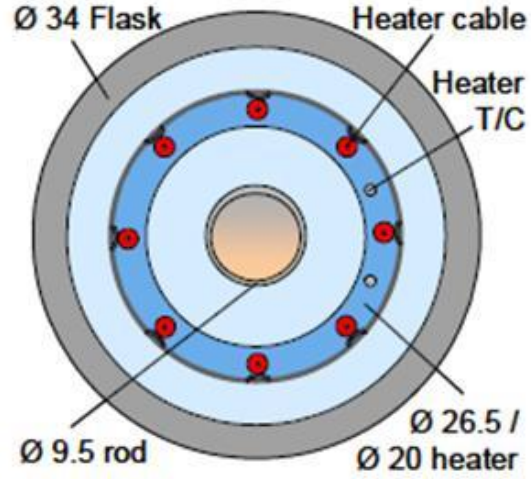

(b)

Figure 1.6: IFA-650 experimental rig and instrumentations

a) Experimental rig and instrumentation [26]; b) axial cross section test rig [16]. Dimensions in (b) are given in [mm].

A single fuel rod is inserted into a pressure flask connected to a water loop and details of the loop of the Halden Reactor are shown in Figure 1.7. A low level of nuclear power generation in the fuel rod is used to simulate decay heat, whereas the electrical heater surrounding the rod is simulating the heat from surrounding rods and it also can be changed during the transient to influence the radiation heat transfer between the rod and the heater thus controlling the PCT.

The rod instrumentation can slightly change depending on the test but it usually consists of:

- Two cladding thermocouples at the upper part of the rod and one at the bottom part. 
- Two heater thermocouples at two different axial elevations.

- A cladding extensometer.

- A rod pressure transducer to measure the rod internal pressure during the transient.

- Coolant thermocouples at the inlet and outlet of the test rig.

- Three distributed vanadium neutron detectors to measure the axial power distribution.

- Two fast response cobalt Self-Powered Neutron Detectors (SPNDs) to monitor rapid flux and power changes.

Before test execution, the reactor is operated for several hours at constant power. After power calibration the procedure to start the LOCA transient is initiated. The procedure consists in a first phase of natural circulation in which the rig is disconnected from the circulation loop (see Figure 1.7). Water flows up between the fuel rod and flow separator (with heater) and down between the flow separator and the flask wall. Following this first phase of natural circulation, the valves that simulate the LOCA are opened and the channel pressure decreases because of the blowdown. The coolant is discharged in the dump tank and the rig is practically emptied in some tens of seconds [27].

The stagnant superheated steam surrounding the test pin provides reduced cooling and the fuel cladding temperature increases quickly. Ballooning and burst usually occur during the heat-up phase. The PCT is reached and held for some time. The test is ended by a reactor SCRAM. The outer loop is reconnected to the rig only several hours after the test to avoid loop contamination. A typical transient response is shown in Figure 1.8.

The Halden LOCA test series IFA-650 has used PWR, BWR and VVER fuels at different burnup, cladding materials, cladding corrosion layer, cladding hydrogen contents, rod filling pressure and free volume space in the upper plenum. An overview of the different tests performed is provided in Table 1.2. A Post Irradiation Examination (PIE) follows the experimental test with destructive and non-destructive tests. For the purpose of fuel fragmentation investigation, the neutron radiography (non-destructive test) and the ceramography (destructive test) provides insights on the status of the fragmented pellets after the test. Due to the different boundary conditions and fuel used for different tests (see Table 1.2), different degrees of fuel fragmentation have been observed. An overview of the PIE results for the different tests can be seen in Table 1.3 [26].

From Table 1.3, the effect of burnup on fuel fragmentation can be clustered as follows: only cracks for fresh fuel (IFA-650.2), consistent fragmentation with fragment size of similar dimension (e.g. IFA-650.11) and fuel close to pulverization with IFA-650.9. 


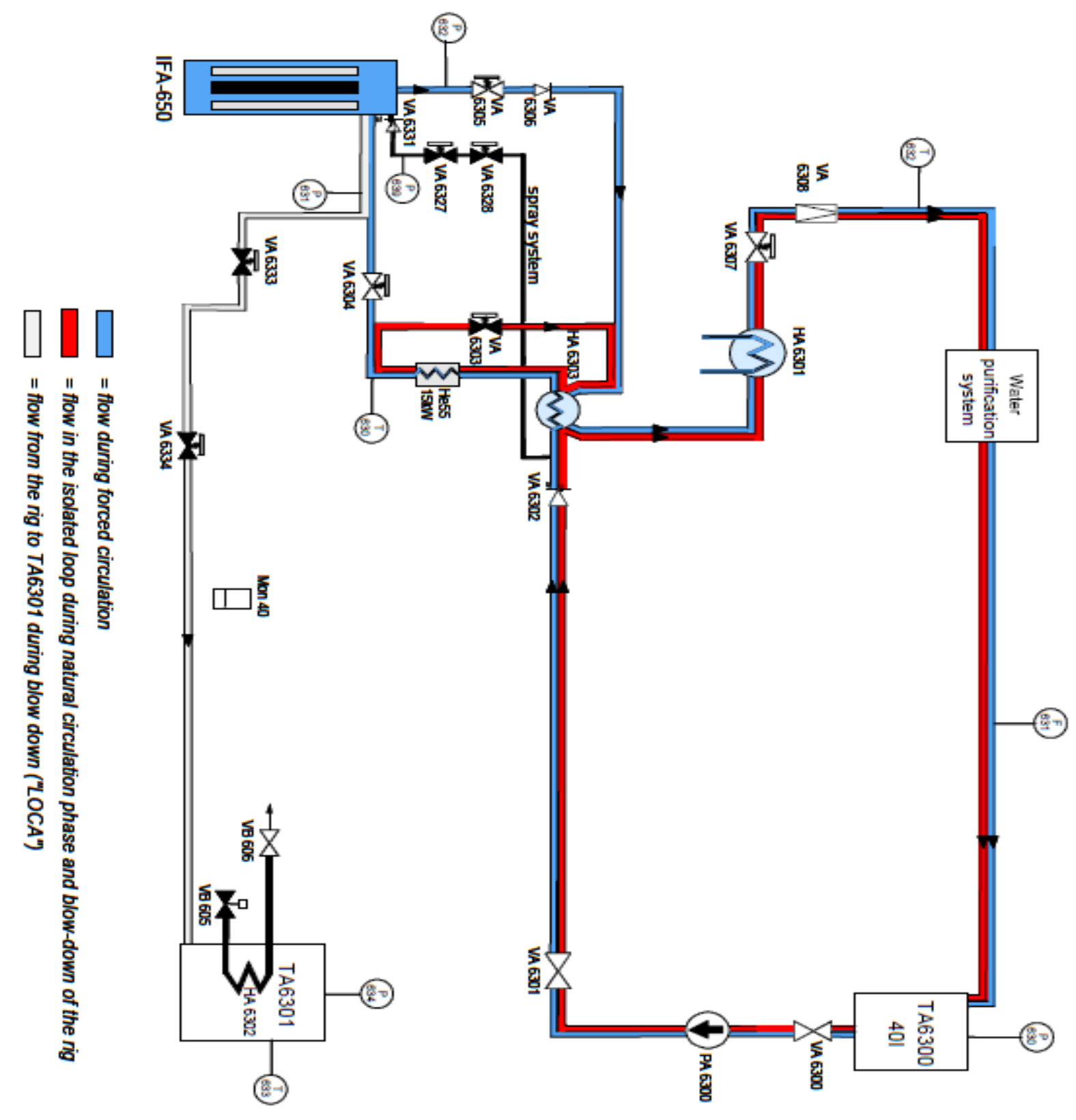


Figure 1.7: IFA-650 loop

Figure from Reference [27] 


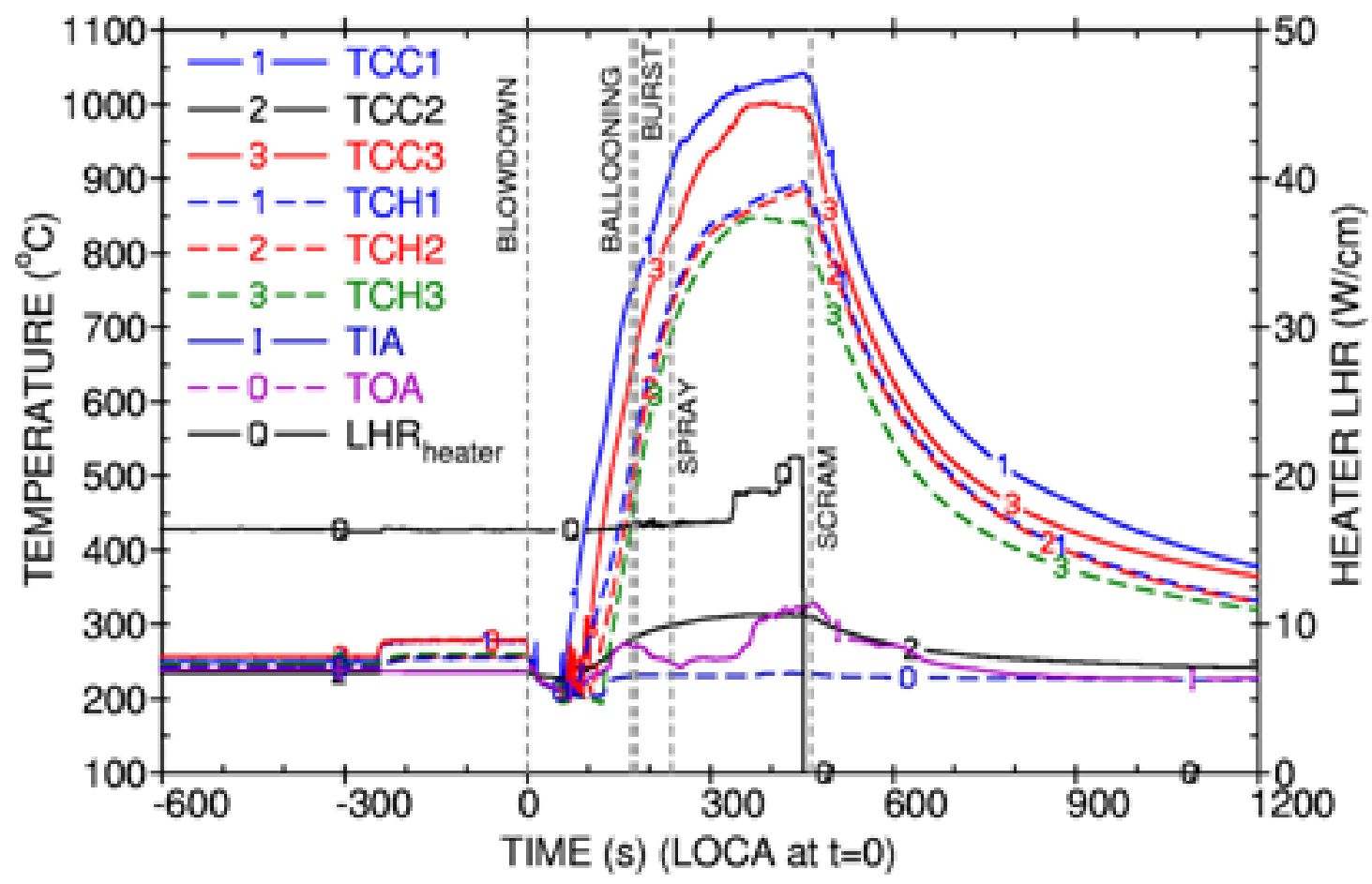

Figure 1.8: Typical IFA-650 transient

Figure from Reference [26]: TCC1, TCC2: top cladding thermocouples; TCC3: bottom cladding thermocouple; TCH1: top heater thermocouple; TCH2: middle heater thermocouple; TCH3: bottom heater thermocouple; TIA: rig inlet coolant thermocouple; $T O A$ : rig outlet coolant thermocouple; $L H R_{\text {heater }}$ Linear heat generation rate heater. 


\begin{tabular}{|c|c|c|c|c|c|c|c|c|c|c|c|c|c|}
\hline $\begin{array}{l}\text { Parameter } \\
\mathrm{s}\end{array}$ & $\begin{array}{l}\text { IFA- } \\
650.1 / 2\end{array}$ & IFA-650.3 & IFA-650.4 & IFA-650.5 & IFA-650.6 & IFA-650.7 & IFA-650.8 & IFA-650.9 & $\begin{array}{l}\text { IFA- } \\
650.10\end{array}$ & $\begin{array}{l}\text { IFA- } \\
650.11\end{array}$ & $\begin{array}{l}\text { IFA- } \\
650.12\end{array}$ & $\begin{array}{l}\text { IFA- } \\
650.13^{* 1}\end{array}$ & $\begin{array}{l}\text { IFA- } \\
650.14^{* 2}\end{array}$ \\
\hline $\begin{array}{l}\text { Target } \\
\text { PCT }\left({ }^{\circ} \mathrm{C}\right)\end{array}$ & \multirow{12}{*}{ Note 1} & 800 & 800 & 1100 & 850 & 1100 & \multirow{12}{*}{ Note 2} & 1100 & 850 & 1000 & 850 & 870 & 850 \\
\hline Fuel Type & & PWR & PWR & PWR & VVER & BWR & & PWR & PWR & VVER & BWR & BWR & BWR \\
\hline $\begin{array}{l}\text { Length } \\
(\mathrm{cm})\end{array}$ & & 50 & 50 & 50 & 45 & 50 & & 50 & 45 & 45 & 38 & 38 & 38 \\
\hline Cycles & & 6 & 7 & 6 & 4 & 3 & & 7 & 6 & 4 & 5 & 7 & 7 \\
\hline $\begin{array}{l}\mathrm{BU} \\
(\mathrm{MWd} / \mathrm{kg} \\
\mathrm{U})\end{array}$ & & 81,9 & 92 & 83 & 55,5 & 44,3 & & 90 & 60 & 56 & 72 & 74 & 71 \\
\hline $\begin{array}{l}\text { Oxide } \\
\text { Thickness } \\
(\mu \mathrm{m})\end{array}$ & & $18-27$ & 10 & $70-80$ & 5 & $<10$ & & $7-8$ & $20-30$ & 5 & 40 & 20 & na \\
\hline $\begin{array}{l}\text { Hydrogen } \\
\text { contents } \\
(\mathrm{ppm})\end{array}$ & & 250 & 50 & 650 & 100 & 44 & & 30 & $150-220$ & 100 & 300 & 300 & na \\
\hline Cladding & & $\begin{array}{c}\text { Zry-4/ } \\
1,47 \% \text { Sn }\end{array}$ & $\begin{array}{c}\text { Zry-4/ } \\
1,47 \% \text { Sn }\end{array}$ & $\begin{array}{c}\text { Zry-4/ } \\
1,47 \% \text { Sn }\end{array}$ & E110 & $\mathrm{LK} 3 / \mathrm{L}$ & & $\begin{array}{c}\text { Zry-4/ } \\
1,47 \% \text { Sn }\end{array}$ & Zry-4 & E110 & $\mathrm{LK} 3 / \mathrm{L}$ & $\mathrm{LK} 3 / \mathrm{L}$ & LK3/L \\
\hline $\begin{array}{l}\mathrm{D}_{\text {out, }} \\
\text { thickness } \\
(\mathrm{mm})\end{array}$ & & $\begin{array}{l}10,75 / \\
0,725\end{array}$ & $\begin{array}{l}10,75 / \\
0,725\end{array}$ & $\begin{array}{l}10,75 / \\
0,725\end{array}$ & $\begin{array}{l}9,62 / \\
0,63\end{array}$ & $\begin{array}{l}9,62 / \\
0,63\end{array}$ & & $\begin{array}{l}10,75 / \\
0,725\end{array}$ & $\begin{array}{c}9,50 / 0 \\
, 57\end{array}$ & $\begin{array}{l}9,13 / \\
0,68\end{array}$ & $9,62 / 0,63$ & $9,62 / 0,63$ & $9,62 / 0,63$ \\
\hline Liner & & 150 & 100 & 150 & No & Yes & & 100 & No & No & Yes & Yes & Yes \\
\hline $\begin{array}{l}\text { Heat } \\
\text { treatment }\end{array}$ & & SRA & SRA & SRA & Standard & Standard & & SRA & SRA & Standard & Standard & Standard & Standard \\
\hline $\begin{array}{l}\text { Fill in } \\
\text { pressure }\end{array}$ & & 40 & 40 & 40 & 30 & 6 & & 40 & 40 & 30 & 20 & 20 & 20 \\
\hline
\end{tabular}

\section{Table 1.2: Overview of IFA-650 tests performed}

Most the information taken from [26]

Note 1: Commissioning Test; Fresh PWR fuel

Note 2: System Check out test with fresh fuel

*1: Information taken from [28]

*2: Information taken from [29] 


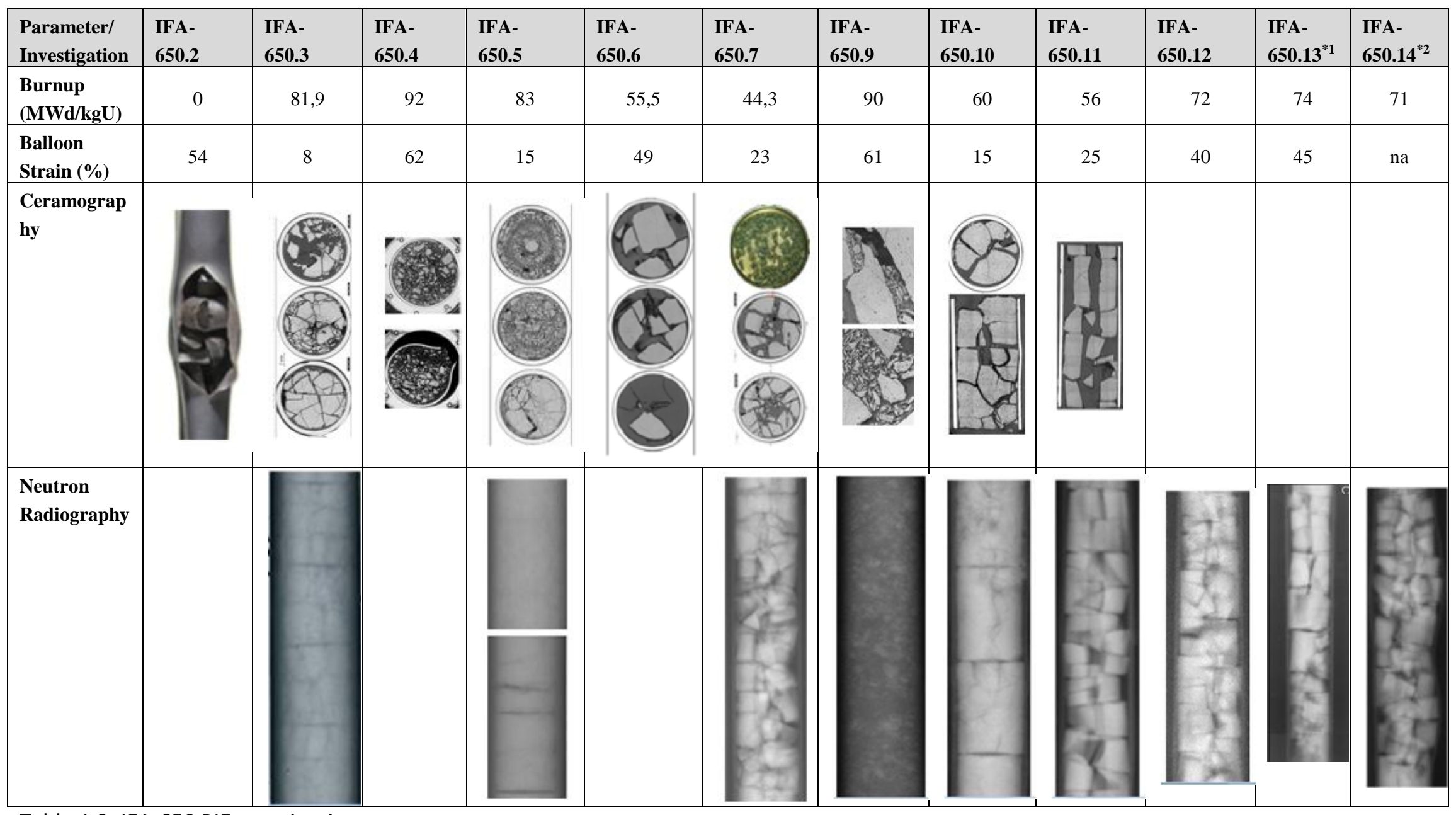

Table 1.3: IFA-650 PIE examination

*1: Information taken from [28]

*2: Information taken from [29]

Materials in part courtesy of HRP 


\subsection{This work}

The latest research outcomes of the IFA-650 test series and the US NRC tests at Studsvik raised further questions for the operation of high burnup fuel because of the observed fuel fragmentation and dispersal. A quantitative assessment of these phenomena is therefore required through additional experimental research and efforts to describe analytically these phenomena to evaluate their safety implications.

The work presented in this dissertation is an experimental research focused on fuel fragmentation during LOCA. Based on observations derived from the study of the IFA-650 tests, the NRC tests at Studsvik and the literature review provided by NUREG-2121, a separate effects experiment was conceived. The test was performed with irradiated fuel rod segments in a hot cell environment with heat provided by an electric oven.

The work had the following primary objectives:

- The design of a LOCA out-of-pile separate effects test

- The study of fuel fragmentation and fragment size distribution under selected boundary conditions

- The study of the fission gas release during LOCA under selected boundary conditions

- The development of a semi-empirical correlation in order to obtain a first order description of the resulting fragment size distribution

The Halden Reactor Project (HRP) was selected as a partner to perform all the activities needed for the successful experiment execution: commissioning trials, rod remanufacturing and handling of irradiated materials inside the hot cell, test execution and post experiment investigations. 


\section{Bibliography}

[1] "Nuclear fuel behavior in Loss-of-coolant Accident Conditions", State-of-the-art Report, OECD, Nuclear Energy Agency 2006

[2] U.S. Code of Federal Regulations, "Reactor Site Criteria," Part 100, Chapter I, Title 10, "Energy"

[3] U. S. Code of Federal Regulations, "Domestic Licensing of Production and Utilization Facilities," Part 50, Chapter I, Title 10, "Energy"

[4] D. Olander, A. Motta, "Light water reactor materials", PSU, Draft 2009

[5] S.N. Aksan, D. Bessette, I. Brittain, F. D'Auria, P. Gruber and others, "CSNI code validation matrix of thermo-hydraulic codes for LWR LOCA and transient", OECD NEA 1987

[6] C. Grandjean, G. Hache, "A state-of-the-art review of past programmes devoted to fuel behavior under loss-of-coolant conditions. Part 3. Cladding Oxidation. Resistance to quench and postquench loads", IRSN 2008

[7] ANSI/ANS-5.1-2005, "Decay heat in light water reactors", American Nuclear Society 2005

[8] N.E. Todreas, M.S. Kazimi, "Thermal Hydraulic Fundamentals, Volume 1", CRC Press 2012

[9] D.A. Powers, R.O. Meyer, "Cladding swelling and rupture models for LOCA analysis", NUREG0630, US NRC 1980

[10] "PWR fuel behavior in design basis accident conditions", CSNI Report 129, OECD NEA, December 1986

[11] G. Hache, H.M. Chung, "The history of LOCA embrittlment criteria", NUREG/CP-0172, 2011, pp. 205-237

[12] "RSK-Leitlinien für Druckwasserreaktoren", RSK-Leitlinien, Ursprungsfassung (3. Ausgabe vom 14. Oktober 1981) mit Änderungen vom 15.11.1996

[13] M. Billone, Y. Yan, T. Burtseva, R. Daum, “Cladding Embrittlement during postulated Loss-ofCoolant Accidents", NUREG/CR-6967, US NRC 2008

[14] "Draft Regulatory Guide DG-1263", U.S. Nuclear Regulatory Commission, Office of Nuclear Regulatory Research

[15] A. Wensauer, "Loss of Coolant Accidents: Current Status of Experimental Verification of Safety Criteria and Safety Assessment", KTG-Fachtag Brennelemente, April 2014

[16] W. Wiesenack, "CSNI Report on Safety Significance of the Halden IFA-650 LOCA Test Results", NEA 2010

[17] E. Kolstad, V. Lestinen, W. Wiesenack, "LOCA Testing at Halden, Trial Runs in IFA-650", IFE/HR/E2003/029, Presented at the Nuclear Safety Research Conference, Washington, October 20-22, 2003

[18] V. Lestinen, "LOCA testing at Halden, first experiment IFA-650.1", HWR-762, March 2004

[19] "Resolution of Generic Safety Issues: Issue 92: Fuel Crumbling During LOCA (Rev. 1) (NUREG0933, Main Report with Supplementes 1-34)

[20] Schemel JH, Papazoglou TP, "Zirconium in the Nuclear Industry", STP681 1979

[21] M. Flanagan, P. Askeljung, "Observation of fuel fragmentation, mobility and release in integral, high-burnup, fueled LOCA tests", Presented at Enlarged Halden Program Group Meeting, Sandefjord, Norway, 2-7 October 2011 
[22] Ralph Meyer, "LOCA Research Information Letter", RIL-0801, US NRC 2008

[23] Patrick A.C. Raynaud, "Fuel Fragmentation, Relocation and Dispersal During the Loss-of-coolant Accident", NUREG-2121, US NRC 2012.

[24] G. Lerchl, H. Austregesilo, P. Schöffel, D. von der Cron, F. Weyermann, "ATHLET Mod 3.0 Cycle A User's Manual“, GRS 2012

[25] "TRANSURANUS handbook", European Commission, JRC Institute for Transuranium Elements 2011

[26] W. Wiesenack, "Overview of HRP LOCA testing", HRP LOCA workshop, Lyon, May 2012

[27] Mirkka Ekk, "LOCA testing at Halden, the third experiment IFA-650.3", HWR-785, OECD Halden Reactor Project 2005

[28] B. Oberländer, W. Wiesenack, "Overview of Halden Reactor LOCA experiments (with emphasis on fuel fragmentation) and plans", Presentation at the NRC public meeting on fuel fragmentation, relocation and dispersal under Loss-of-Coolant Accident (LOCA) conditions, March 13-14, 2014

[29] P. Tradotti, "LOCA testing at Halden, the BWR fuel experiment IFA-650.14", Enlarged Halden Programme Group Meeting, September 2014, Røros, Norway 


\section{Development of the separate effects test}

\section{Contents}

2 Development of the separate effects test

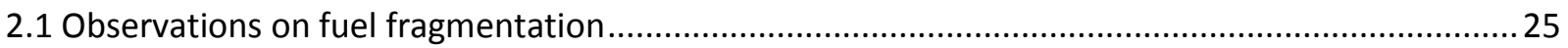

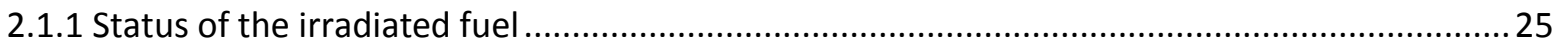

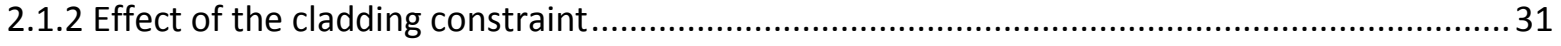

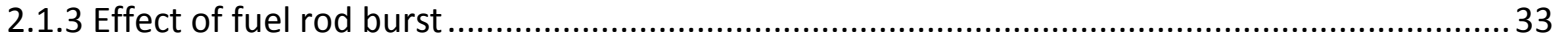

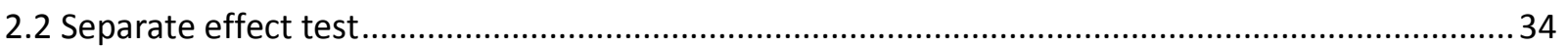

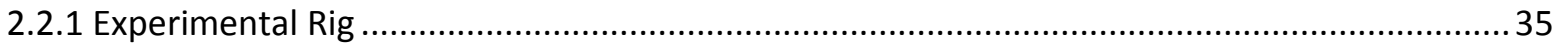

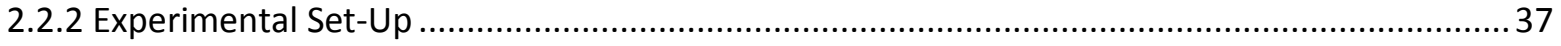

2.2.3 Modeling of the Experimental Rig …................................................................................ 42

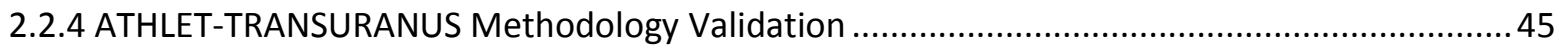

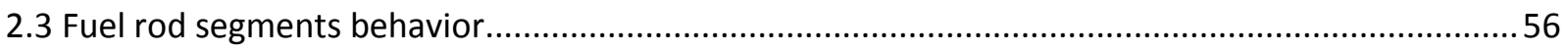

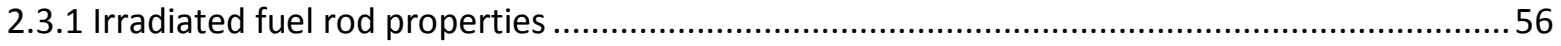

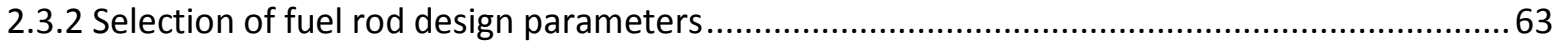

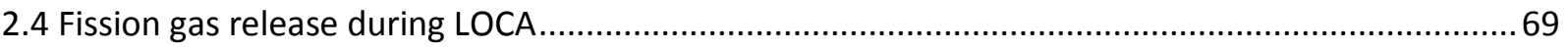

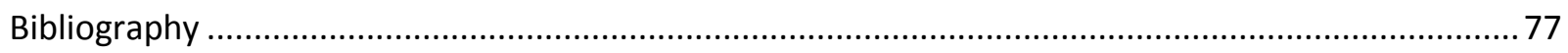

This chapter describes the design phases of the separate effects test. Observations on the Halden LOCA Test [1], the Studsvik Test [2] and the literature review of LOCA NUREG-2121 [3] were the spark of the experimental investigation as described in 2.1. 
Based on these observations a conceptual design was conceived as illustrated in 2.2 and a methodology was developed to simulate the experiment by means of the calculation codes ATHLET [4, 5] and TRANSURANUS [6].

Key information on the irradiated fuel segment used in the test and its properties calculated with TRANSURANUS are provided in 2.3. TRANSURANUS was also used to determine the fuel rod parameters required to manufacture the three rod segments.

An additional objective of the test was the study of the fission gas released during the transient. The fission gas release during IFA-650.12 is studied in 2.4 as basis for the prediction of the release expected from the rod segments during the separate effects test.

\subsection{Observations on fuel fragmentation}

The literature described in Chapter 1 is a source of information to investigate fuel fragmentation during LOCA. As is shown in Table 1.1, fuel fragmentation was rarely the main focus of the investigations, but rather a "by-product" of the experiments. Moreover, the experimental set up and the pre- and postexperiment investigations were not conducive for consistent and structured experimental observations on fuel fragmentation.

The separate effects test design is the result of observations on fuel fragmentation collected and analyzed during the research investigation. These observations have been clustered into 3 different topics: status of the irradiated fuel (2.1.1), effect of the cladding constraint (2.1.2), and effect of fast depressurization due to cladding rupture (2.1.3).

\subsubsection{Status of the irradiated fuel}

During irradiation, several burnup induced phenomena occur in the fuel and continuous attempts are made to improve the modeling of these phenomena [7]. Phenomena having an influence on the matter of study are underlined.

- Fuel Cracks: $\mathrm{UO}_{2}$ is a ceramic material and consequently the thermal conductivity $\left(\lambda_{U O_{2}}=\right.$ $4,2 \mathrm{~W} / \mathrm{m} \cdot \mathrm{K}$ at $\left.500^{\circ} \mathrm{C}\right)$ and the coefficient of thermal expansions $\left(\alpha_{U O_{2}}=10 \cdot 10^{-6} 1 / K_{K}\right)$ are rather low. This leads to a steep temperature gradient and to a tendency for crack formation. This occurs during irradiation: thermal stresses are induced in the fuel due to a steep radial temperature profile and its changes in power ramps causing the fuel pellet to crack with the number and strength of cracks depending on the power history. Figure 2.1 shows a typical section of LWR fuel after irradiation with radial and longitudinal cracks at room temperature. 
The status of the fuel after irradiation in terms of cracks is very important because it is the fingerprint of the fuel before the starting of the LOCA heating up phase. In this phase the center of the pellet rapidly cools down generating compressive stresses while the outer part of the pellet rapidly heats up generating expansive stresses.

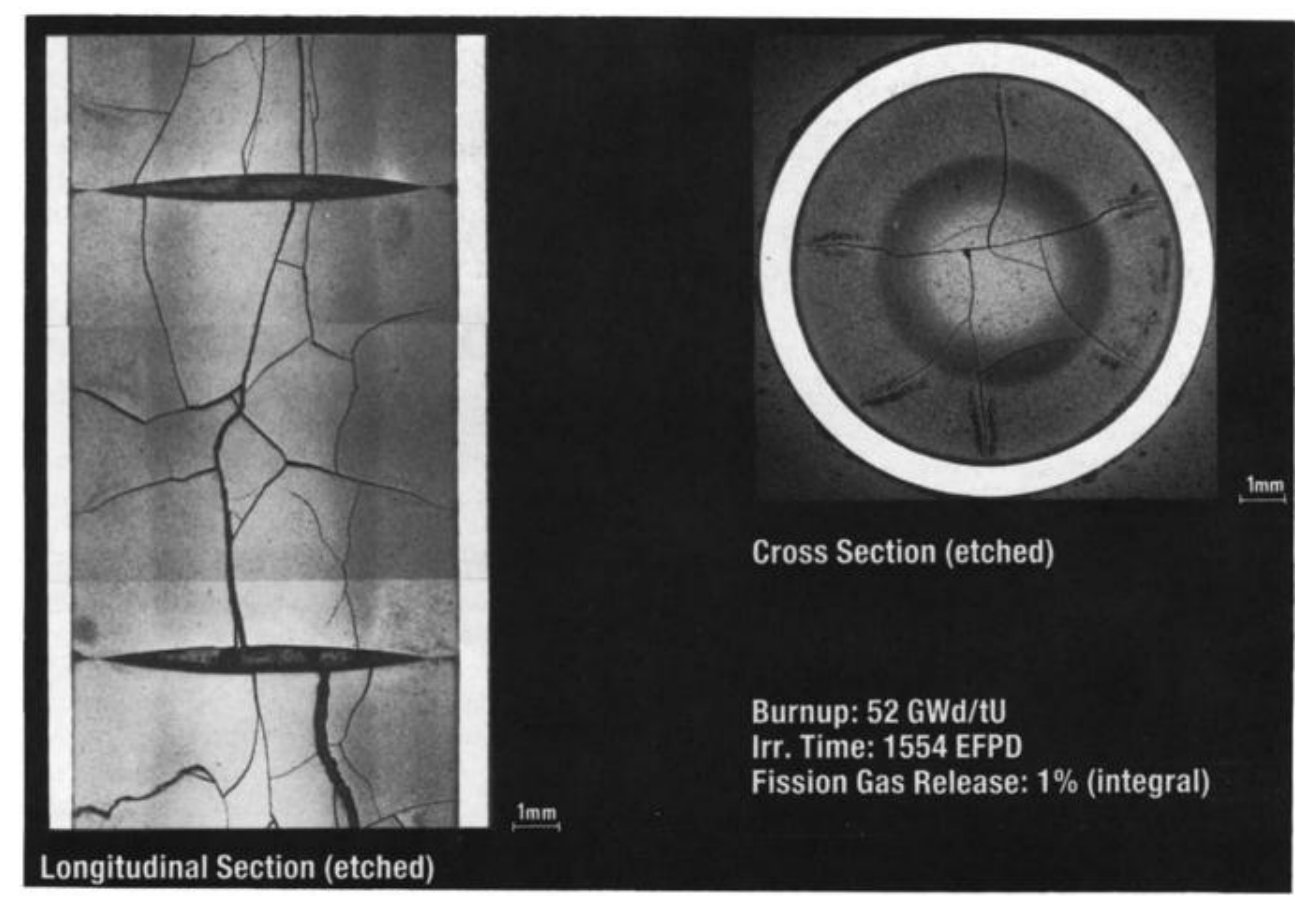

Figure 2.1: Typical LWR fuel after irradiation Figure from Reference [8]

- Fuel swelling: Fuel swelling is caused by the overall increase in the volume of matter when heavy atoms are fissioned and non-soluble fission gases accumulate at grain boundaries in intergranular fission bubbles. Specifically, complex fission product chemistry is brought into action with the formation of solid solution, metallic precipitates, to a lesser extent also non-metallic precipitates, and - at temperatures of $1200^{\circ} \mathrm{C}$ - microscopic and submicroscopic rare gas bubbles [9]. Due to fuel swelling and fuel relocation, and because of the Zircaloy cladding creep-down, the closure of the gap and a contact force between cladding and fuel pellets builds up. The gap status influences fuel fragmentation because of the following: 1) the fuel gap closure changes the heat transfer coefficient in the gap between the fuel and the rod with consequent influence on the fuel thermal stress; 2) a gap closure diminishes the level of communication between different axial regions of the fuel and if burst occurs, depressurization rates at different axial locations of the fuel may be very different and 3) when fuel and cladding interact and under specific irradiation conditions [10], there is a formation of a fuel-cladding bonding layer with specific chemical, physical and mechanical properties: during a LOCA transient in presence of cladding ballooning, the detachment of the bonding layer acts as a radial fuel fragmentation force. 
- Micro-structural changes and high burnup structure: During the first cycles of irradiation at high linear generation rate, moderate equiaxed growth of the $\mathrm{UO}_{2}$ grains is usually observed in the centre of LWR fuel rods [8]. The local burnup at the rim of the $\mathrm{UO}_{2}$ pellet can be 2-3 times higher than the average pellet burnup, depending on the specific irradiation conditions. This is due to the neutron-self shielding whereby in a thermal neutron spectrum there is a higher density of epithermal neutron resonance absorption in $U_{238}$ nuclei at the radial outer edge, or rim, of the $\mathrm{UO}_{2}$ fuel pellet than in the pellet center. This also results in a local enrichment in fissile plutonium at the pellet rim [10]. During in-pile irradiation, starting at local burnup around $65 \mathrm{MWd} / \mathrm{kgHM}$ and at the irradiation temperature below about $1000^{\circ} \mathrm{C}$, the fuel undergoes a restructuring process, which results in the appearance of a new fuel morphology; at a local burnup of circa $75 \mathrm{MWd} / \mathrm{kgHM}$ the fuel appears fully transformed. This structure, called High Burnup Structure (HBS) is characterized by grain subdivision and redistribution of fission gases and extended defects. The original grains, with a typical size of 10 micron, are subdivided into sub micro-grains with a size of 0.1-0.3 micron as shown in Figure 2.2. The start of the formation of this new fuel structure has an important influence in fuel fragmentation. In fact, experiments with irradiated fuel at high burnup have shown, after the test, very small fragments and powder at the bottom of the fuel rod or dispersed outside the fuel rod.

- Fission gas creation and fission gas release: During irradiation in a light water reactor, long lived gas atoms are produced by fission. Due to the small thermo-dynamic solubility of noble gases [11], they precipitate in the $\mathrm{UO}_{2}$ matrix. The gas atom, being in a dynamic solution, diffuse to grain boundaries, having as a driving force for the process the concentration gradient of the gas atoms. The gas atoms reaching the grain boundaries form gas bubbles on grain surfaces as well as at grain edges. The growth of grain face bubbles influences the rate of development of grain edge porosity which eventually forms an interlinked network of tunnels and provides thereby an easy escape path for gas atoms produced deep in the body of the fuel. Of course, the asfabricated open porosity which decreases during irradiation due to densification, also contributes to the channel system, through which fission gas is released [12]. With the creation of the high burnup structure, the fission gas is removed from the fuel matrix, and is retained in high concentration in micron-sized closed pores with a porosity that can exceed 20\% [13]. Consequently at the end of irradiation, part of the fission gas produced remains within the grains, part in the grain boundaries, part is transferred to the porosity of the developed high burnup structure and part is released into the fuel rod plenum. To the purpose of studying fuel fragmentation, it is very important to correctly predict where the fission gas produced is distributed within the pellet. Within the LOCA heating up phase, the gas concentrated in the matrix, pores and bubble increase its pressure and act as matrix fragmentation force. The weaker the fuel matrix's structure because of defects and micro-structural transformation during irradiation, the stronger the fragmentation effect given by the gas pressure increase. 


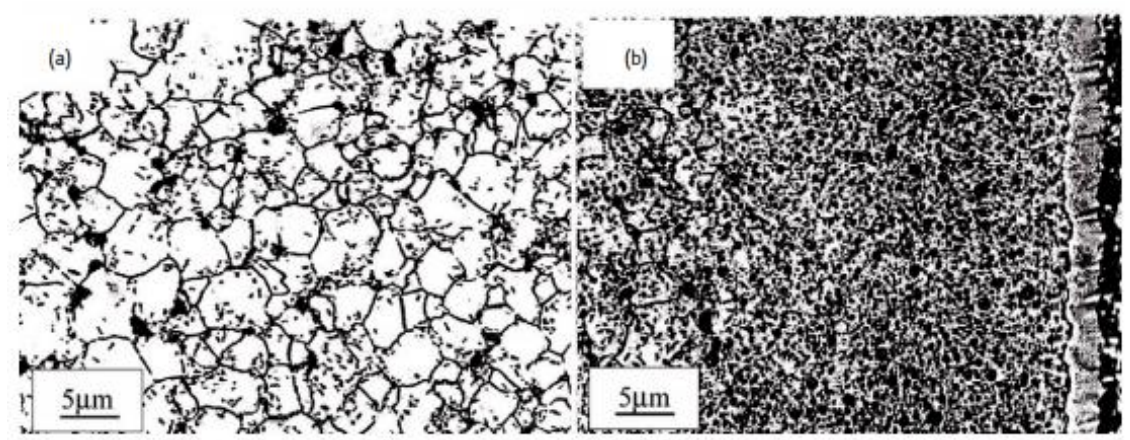

Figure 2.2: Ceramography of a cross-section of a LWR pellet Figure from Reference [32]; burnup of circa $65 \mathrm{MWday} / \mathrm{kgHM}$

(a) Unrestructured fuel at the intermediate radial region

(b) HBS at the rim region of the fuel pellet

Figure 2.3 shows fuel cross section after LOCA tests as function of the fuel average burnup. For the purpose of studying fuel fragmentation, it is important to compare fuel axial sections with a comparable axial distance to the burst location. Sub-Chapters 2.1.2 and 2.1.3 are explanatory concerning this statement.

The cross sections belong to axial zones near the burst location and they can be clustered in three main regions:

- Fuel Cracks Region: up to approximately 40-50 MWd/kgU, fuel cross sections after a LOCA test have big cracks and the fuel is fragmented in a countable number of fragments with most of particle sizes above about 3-4 $\mathrm{mm}$. In addition, within this range, it seems that the burnup value is invariant.

- Coherent fuel fragmentation: between $40-50$ and about $70-80 \mathrm{MWd} / \mathrm{kgU}$ it is possible to observe small fragments with a narrow distribution with a reduction of particle sizes with increasing fuel burnup.

- Pulverization region: above $70-80 \mathrm{MWd} / \mathrm{kgU}$, the cross section shows very small fuel fragments and powder.

Figure 2.4 shows the increase of thickness of the high burnup structure as function of the fuel average burnup predicted by TRANSURANUS for a generic LWR fuel cycle. In TRANSURANUS, the beginning of the development of the HBS is identified by the boundary "transition HBS" at local burnup of about 65 $\mathrm{MWd} / \mathrm{kgU}$ (corresponding to a fuel average burnup of about $40 \mathrm{MWd} / \mathrm{kgU}$ ), while the full transformation is identified by the boundary "fully developed HBS" at a local burnup of approximately $75 \mathrm{MWd} / \mathrm{kgU}$ (corresponding to a fuel average burnup of about $55 \mathrm{MWd} / \mathrm{kgU}$ ). Since the transformation of the fuel matrix into the HBS requires an irradiation temperature below $1000^{\circ} \mathrm{C}$, the hot central zone of the fuel is not subject to the restructuring and this is shown in Figure 2.4 with the saturation conditions occurring at approximately $70-80 \mathrm{MWd} / \mathrm{kgU}$ for the "transition HBS". It is interesting to note that the two boundaries at $40-50$ and $70-80 \mathrm{MWd} / \mathrm{kgU}$ of Figure 2.3 correspond to the starting and the completion of the development of thickness of the "transient" high burnup structure in Figure 2.4. 
The transition between the "fuel cracks" region to the "coherent fragmentation" region can be explained as a combination of higher burnup (bigger number of cracks before the LOCA test) and the starting development of the HBS, with the appearance after the test of some fine particles. The integral mass of these fine particles tends to increase with the burnup approaching the pulverization region, in which the thicknesses of the "transient" and "fully developed" HBS are stabilized. In the pulverization region the integral mass of fine particles is relevant.

It is important to underline that the considerations are valid for cross sections of the fuel very close to the burst region. In 2.1.1 and 2.2.2, experimental observations in terms of fuel fragmentation as function of external constraints are provided with observations of fuel cross sections far from the burst region. The identified external constraints are the geometrical constraint provided by the cladding and the constraint provided by the rod internal pressure.

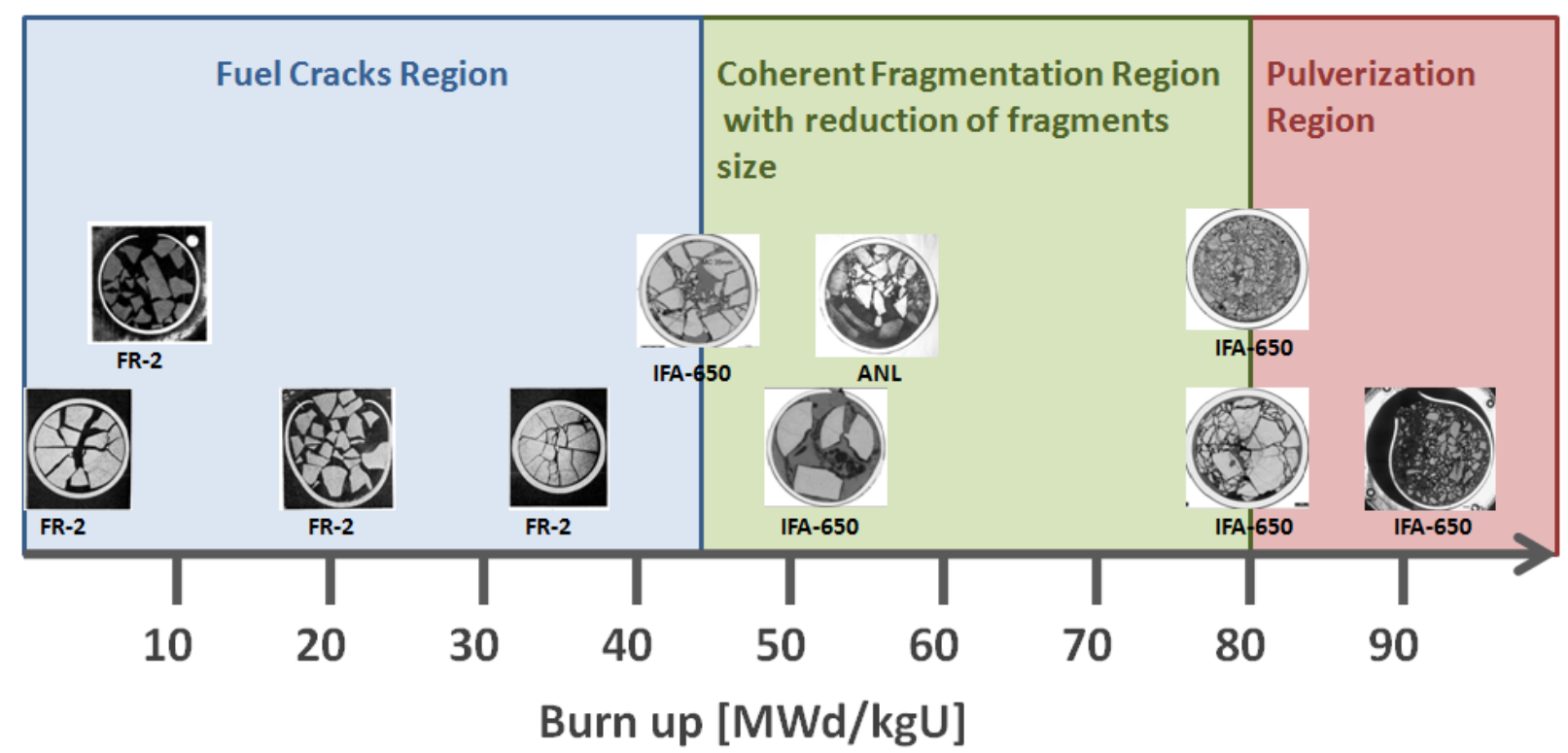

Figure 2.3: Ceramography of pellet's cross-section after LOCA function of BU Pictures taken from [3] and internal material presentation courtesy of HRP 


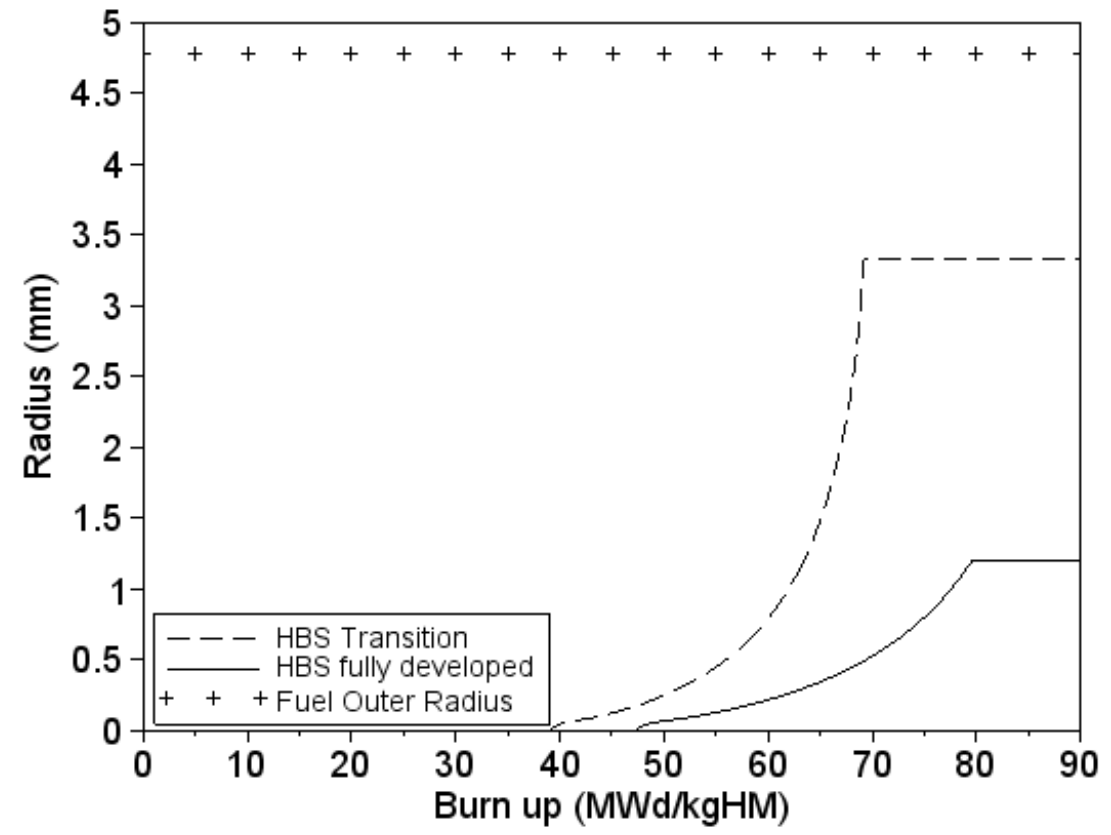

Figure 2.4: TRANSURANUS simulation of development of "transition" and "fully developed" HBS Figure developed with TRANSURANUS for a generic PWR power history 


\subsubsection{Effect of the cladding constraint}

The role of the geometrical constraint of the cladding in the fragmentation process can be found in several experimental results by looking at different fragmentation degrees occurring at different axial fuel locations that experienced different axial cladding deformations.

Figure 2.5 and 2.6 show pellet cross sections at different axial locations as function of the cladding deformation measured after the tests IFA-650.5 and IFA-650.3. In Figure 2.5, the difference between cross sections 1-5 and 6-7 is evident: strong fuel fragmentation with very fine particles in accordance with very large cladding deformation (cross sections 1-5) and only fuel cracks and minor area of fuel fragmentation in step with small cladding deformation (cross sections 6-7). In Figure 2.6, a similar pattern can be observed comparing the cross section at the axial location of about $250 \mathrm{~mm}$ (maximum cladding deformation) and $100 \mathrm{~mm}$ : the fragmentation with larger cladding deformation is stronger than the fragmentation with smaller cladding deformation.

Figure 2.7 shows a similar behavior for the ANL tests [3]: the maximum fragmentation is observed for the cross section (a) corresponding to $15-35 \%$ cladding strain. The fragmentation strength slightly decreases for a smaller cladding strain as shown by the cross section (b) at $2-4 \%$ strain. It is also interesting to observe that the cross section in correspondence to negligible strain (c) is very similar to the cross section of the fuel of an archive pellet that was not subject to the LOCA test (d).

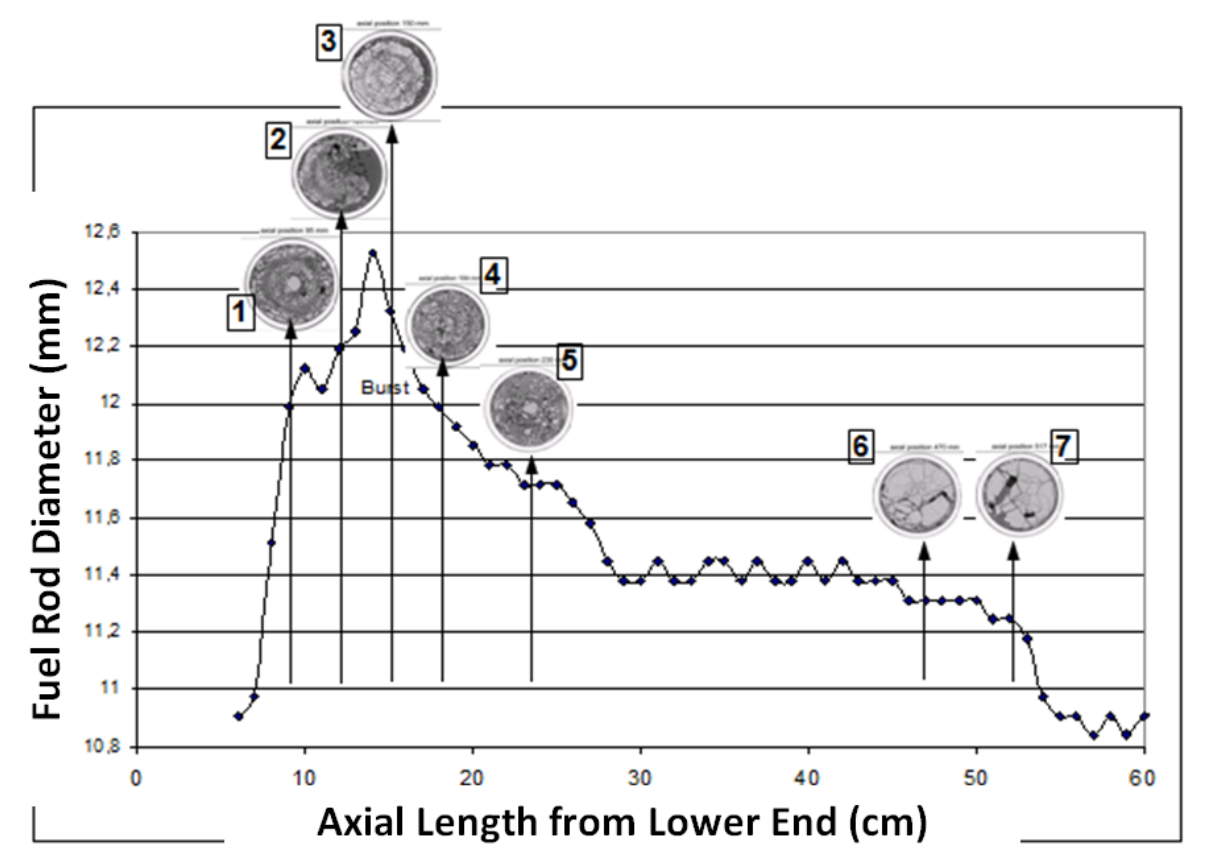

Figure 2.5: IFA-650.5 pellet's cross sections as function of axial position Elaboration of internal material courtesy of HRP 


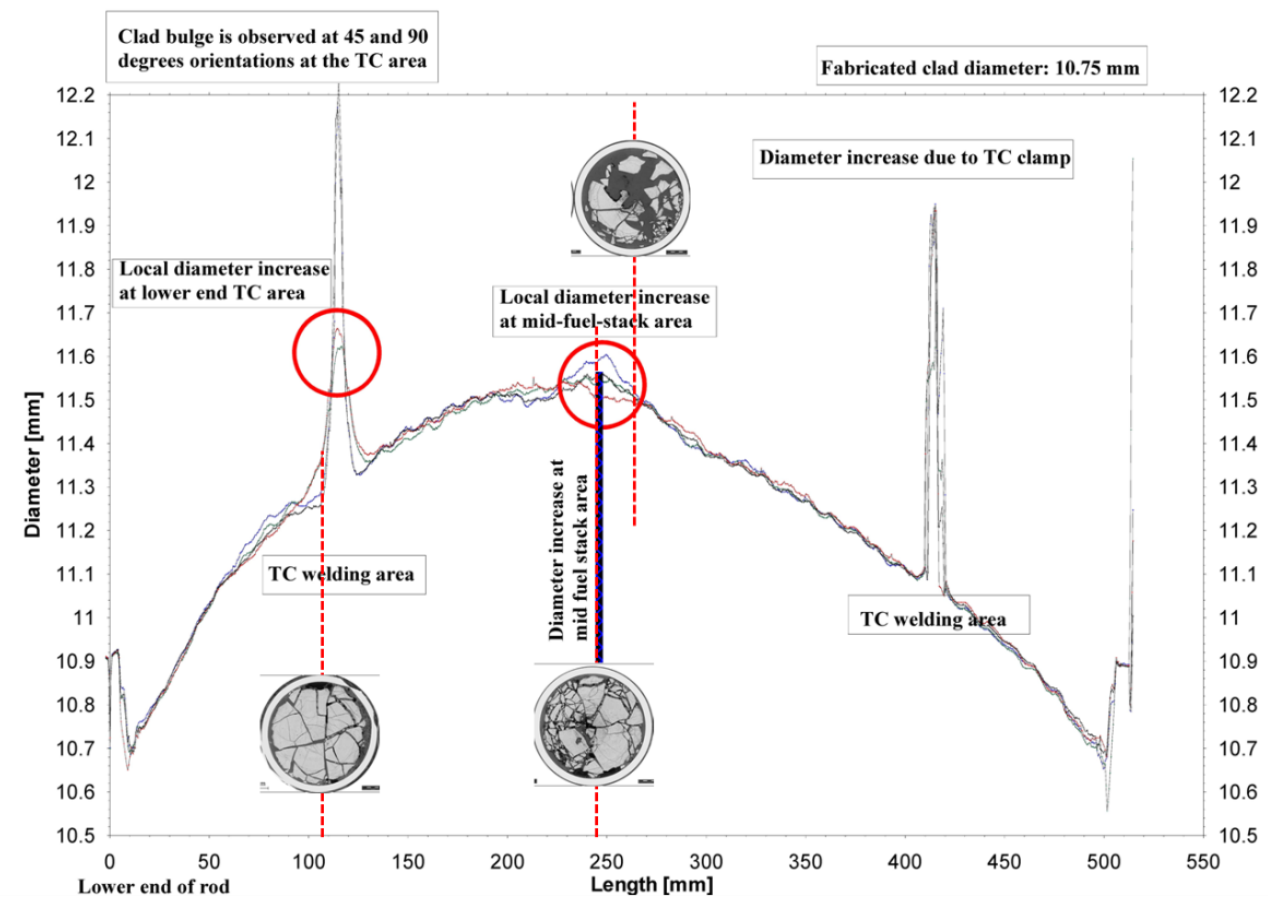

Figure 2.6: IFA-650.3 pellet's cross sections as function of axial position Elaboration of internal material courtesy of $H R P$

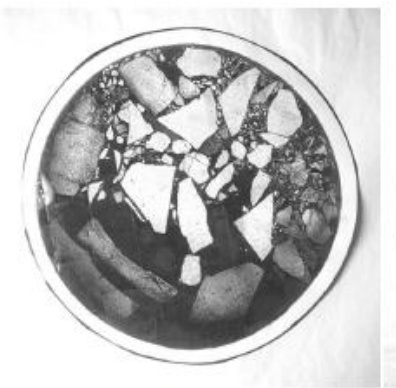

(a)

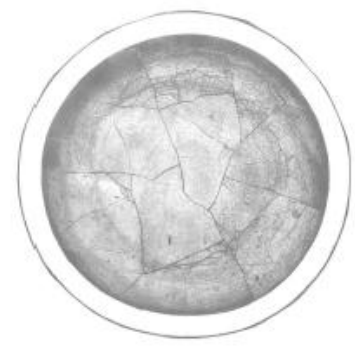

(c)

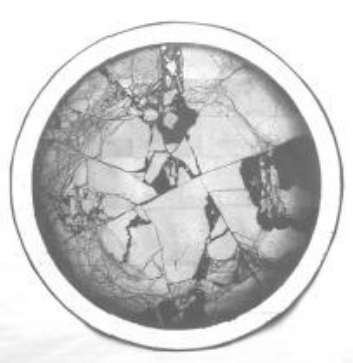

(b)

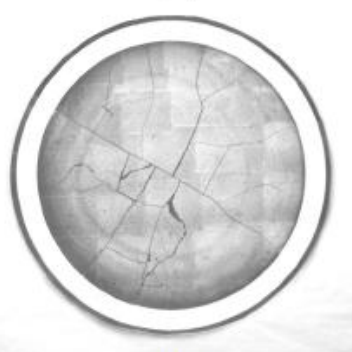

(d)

Figure 2.7: PIE ANL LOCA test at $56 \mathrm{MWd} / \mathrm{kgU}$

Figure from Reference [3]: (a) $12 \mathrm{~mm}$ above rupture (15-35\% strain); (b) $50 \mathrm{~mm}$ above rupture (2-4\% strain);(c) $130 \mathrm{~mm}$ below rupture (strain negligible); (d) prior LOCA testing 


\subsubsection{Effect of fuel rod burst}

The rod internal pressure is considered an external constraint to fuel fragmentation. When the fuel rod bursts, the burst region experiences a strong depressurization and this constraint is removed. The bigger the initial internal rod pressure at the time of burst, the stronger the constraint degradation will be. Different axial locations of the fuel experience different depressurization rates depending upon the distance to the burst location and to the level of communication between different axial regions as described in 2.1.1.

Often the location of maximum cladding deformation corresponds to the location of cladding burst and consequently it is challenging to separate the effect given by the removal of the geometrical constraint of the cladding and of the decrease of rod internal pressure. Assuming a very fast depressurization rate for the region of the fuel close to the burst location, a measure of the rate of depressurization at different axial locations of a full length fuel rod may be extremely challenging.

Evidence that the depressurization rate along the axial length of the fuel is different can be found looking at the measurement of the pressure transducer installed at the top of the IFA-650 experimental rig. Figure 2.8 shows the pressure measurements for IFA-650.3 and IFA-650.4. With the burst occurrence, a very fast depressurization was measured for IFA-650.4 while IFA-650.3 shows a slower depressurization. In particular, for IFA-650.3, about 60 seconds were needed to depressurize the top part of the experimental rig to the fuel rod external pressure. It should be underlined that the pressure transducer was located at the same distance from the top of the rod for the two experiments and the difference in burst locations is considered to not play an important role considering the different order of magnitude observed in Figure 2.8. It is therefore an indication that the level of communication between different axial regions can determine different axial depressurization rates and due to the key role that may play with a full length fuel rod, it should be considered.

Figure 2.9 is pointing also in the direction of an effect of the depressurization rate in strengthening the fragmentation of the fuel for IFA-650.11 (burst location at $210 \mathrm{~mm}$ ). In this case the neutron radiography is used to prove that the fuel tends to fragment more near the location of the burst, while the fragmentation tends to be negligible at the end sides of the fuel. It should be underlined that also for the two cases of below, it is difficult to separate the effects of the cladding deformation and of the depressurization rate. 


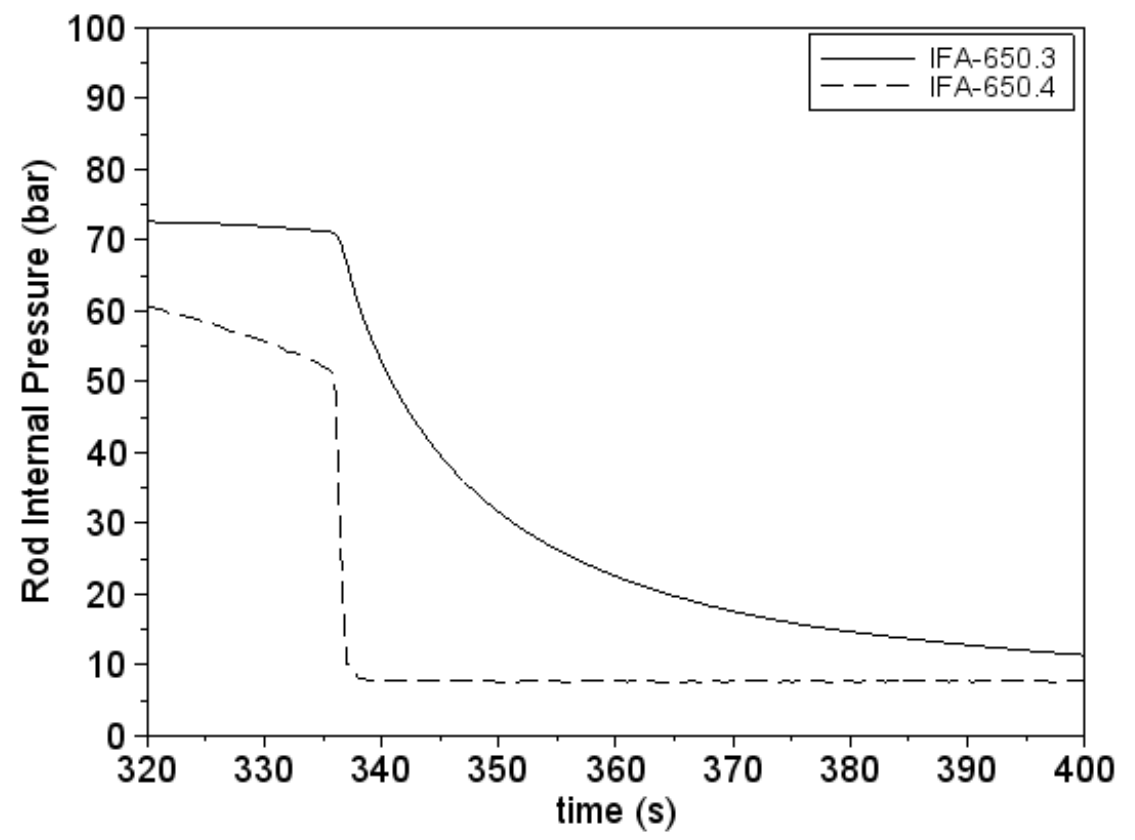

Figure 2.8: Comparison rod inner pressure measurements for IFA-650.3 and IFA-650.4 Elaboration of internal material courtesy of HRP

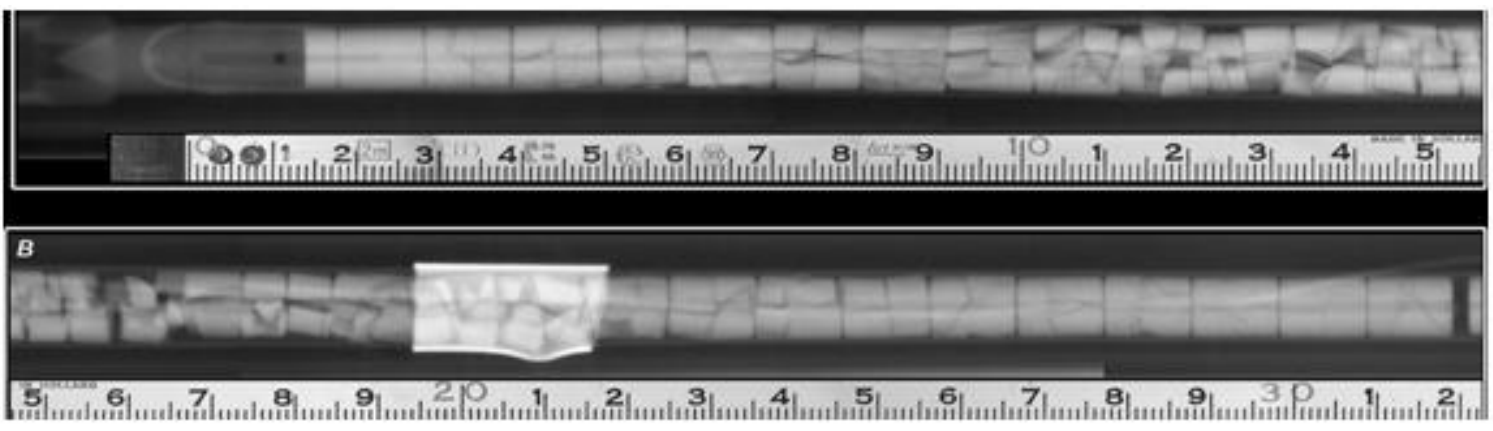

Figure 2.9: Neutron Radiography IFA-650.11

Elaboration of internal material courtesy of HRP

\subsection{Separate effect test}

The observations made in 2.1 were the spark of the research investigation and the basis foundation for the design of the separate effects test. The main characteristics of the test are: the use of a fuel segment irradiated in a commercial nuclear power plant, heating provided by the use of an electric oven and selected boundary conditions. The design of the experimental rig started with a conceptual design that was adapted to the Halden Reactor Project hot cell, tools and laboratories and that was further 
improved based on the outcomes of a planned commissioning campaign needed to test the experimental rig and set-up.

A methodology was developed to model the experimental rig in order to predict the behavior of the fuel rod segments during the test. The methodology and tools were validated against available LOCA experiment data.

\subsubsection{Experimental Rig}

In line with the effects investigated in 2.1, the separate effects test was developed with the idea of studying fuel fragmentation of relatively high burnup fuel and the three following effects:

Effect 1. Fuel fragmentation removing the constraint provided by the cladding.

Effect 2. Fuel fragmentation removing the constraint provided by the cladding and the rod internal pressure.

Effect 3. Fuel fragmentation keeping the constraint provided by the cladding.

The first conceptual design draft of the potential separate effect test is shown in Figure 2.10.

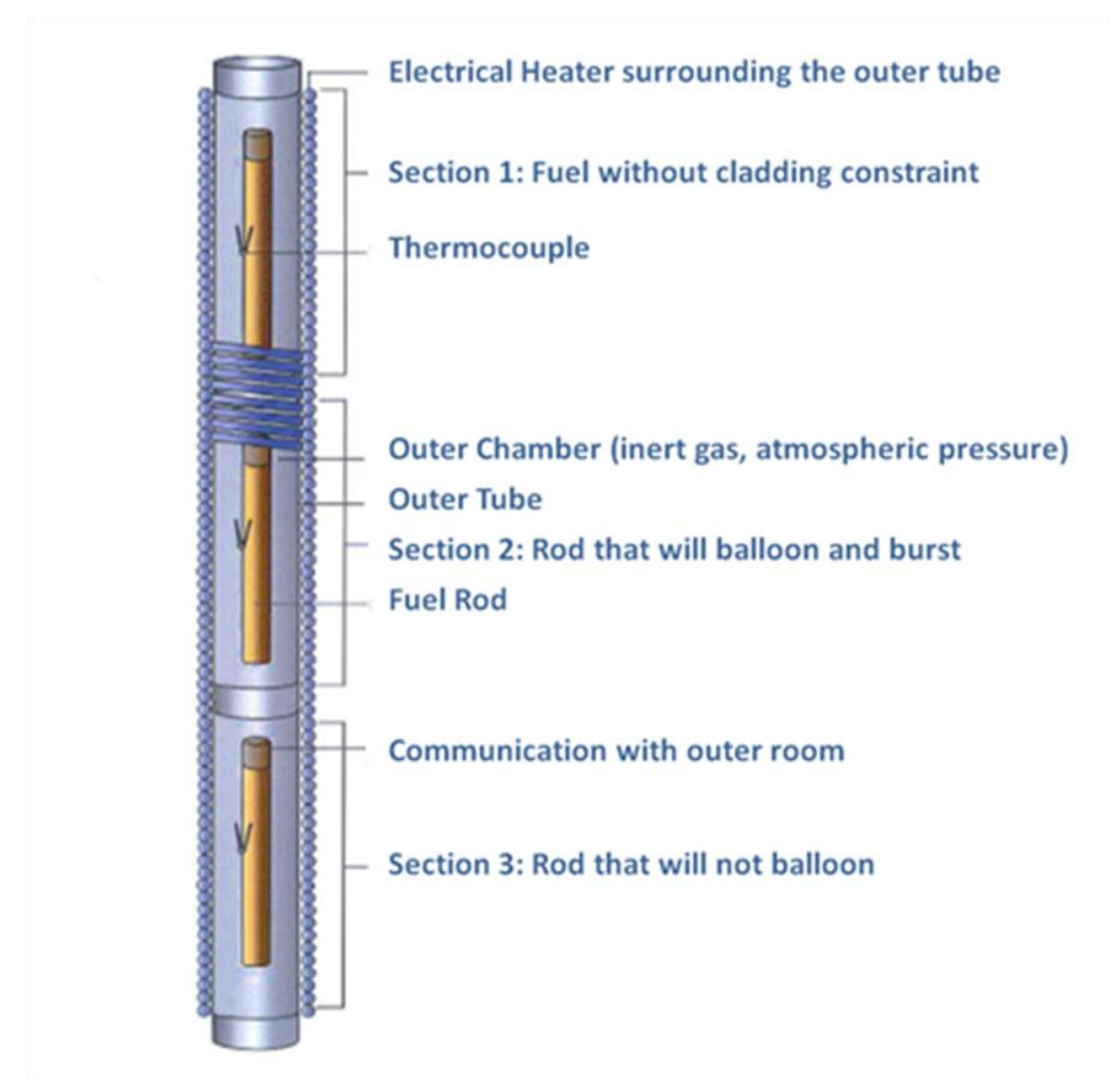

Figure 2.10: Preliminary design based on the conceptual separate effect test 
The draft conceptual design consisted of three irradiated fuel segments surrounded by an outer tube mechanically connected to an electric heater to simulate the LOCA heating up. The three outer rod environments were thought to be not in communication. Thermocouples installed on the cladding would have measured the temperature during the transient.

The three rods design was conceived as follows:

Section 1. The removal of the geometrical constraint given by the cladding to be obtained by weakening the cladding with an axial cut.

Section 2. The removal of the constraint given by the cladding and the rod internal pressure to be obtained by ballooning and burst of the fuel rod.

Section 3. Keeping the geometrical constraint of the cladding and to prevent cladding ballooning with a hole in the upper plenum of the cladding as a channel of communication between the inner and the outside of the rod.

This first draft represented well the conceptual idea of the test. However, the experiment reached a first level of maturity after an investigation on different alternatives to obtain the heat needed to simulate a LOCA condition. As a result of the investigation, an electric oven designed to reach temperatures of about $1200^{\circ} \mathrm{C}$ was considered to be the most feasible solution to produce the heat, in particular because such oven was already available at the HRP laboratories. Using a main oven to provide heat implied the use of capsules to surround each of the three rod segments. A bottom and top structure of connection of the three capsules was manufactured to facilitate the operation of dropping the three capsules inside the oven. Since the oven had a certain axial temperature profile, an alternative spatial configuration of the three rods was found as shown in Figure 2.11 to ensure that the three capsules were heated with a similar heat flux.

The boundary conditions needed to obtain the three effects of above were also revised for Rod 1 and Rod 3, while the design of Rod 2 remained untouched.

- $\quad$ Rod 1: The partial axial cut of the cladding to weaken it and hinder its potential function as a constraint was considered a challenging operation. The behavior during the heating up would have been very difficult to predict and additionally, the quantification of this sluggish constraint would have been ambitious. Moreover, the cut would have introduced a singularity and made the rod asymmetric adding additional challenges for potential simulation of the transient with 1D or 2-D codes. It has been therefore decided to study the effect of removal of the cladding constraint through a design of the upper plenum volume and initial pressure so to obtain ballooning of the cladding without rod failure.

- Rod 3: it was decided that the communication hole between the rod upper free volume and the environment of the outer tube surrounding of the rod was not necessary. Since the heating is provided by an external heater, the structure of the rod is always at slightly lower or equal temperature of the outer tube and consequently, if the initial pressure of the two environments (rod internal pressure and pressure of the capsule) is similar, no ballooning is foreseen because the differential pressure (rod internal pressure minus capsule pressure) is always slightly 
negative or negligible. To minimize the effect of fission gas release during the test a very large upper free volume can be designed.

\subsubsection{Experimental Set-Up}

The oven used in the experiment is shown in Figure 2.11 a). Additional details on the oven are given in 3.1. The oven does not have a constant axial temperature profile; consequently it was considered more appropriate to locate the three capsules at the same axial location. Figure 2.11 a) shows three dummy capsules located inside the oven that were used to study the feasibility of the set up. The three dummy capsules were made of stainless steel and a dummy stainless steel rod was installed inside each capsule as shown in Figure $2.11 \mathrm{~b}$ ). On the dummy capsules and rods a series of thermocouples were attached. The dummy capsules and rods have been manufactured to perform a preliminary commissioning campaign with the following objectives:

- Determine the procedure to start the heating up of the capsule.

- Verify the repeatability of the results performing different runs.

- Prove that there is an equal heating up rate for the three capsules and a uniform azimuthal temperature distribution of the capsules and of the cladding.

- Quantify the heating up rate of the cladding.

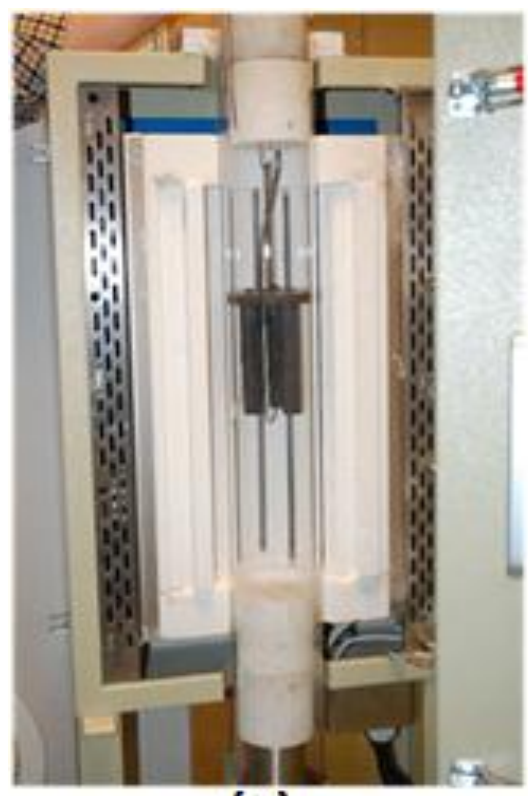

(a)

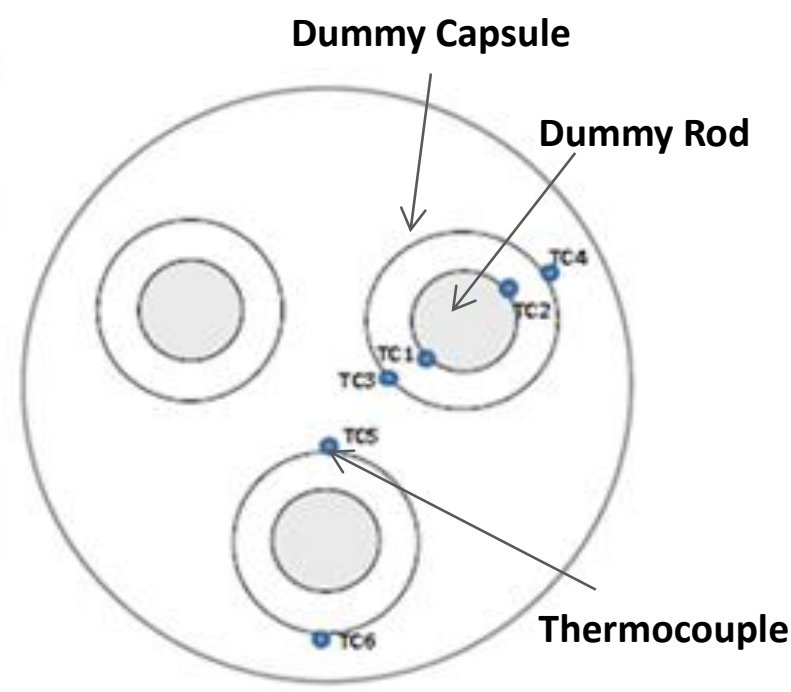

(b)

Figure 2.11: Details of the experimental set-up and experimental rig

Figures from the first commissioning run at the HRP: a) Oven used to simulate the heating up; b) axial cross section of the experimental rig 
The experimental procedure to start the heating of the capsule and consequently of the fuel rod is very important because the target is to represent a heating rate as similar as possible to other LOCA tests and/or simulation of a postulated LOCA transient in a commercial reactor. Two possible procedures were identified:

- Run 1: Locating the three capsules inside the cold oven and select a target temperature close to real reactor condition (about $300^{\circ} \mathrm{C}$ ). When the temperature of the three capsules' structure is stable at $300^{\circ} \mathrm{C}$, set the target temperature of the oven at $900^{\circ} \mathrm{C}$;

- Run 2: Bring the oven to a target stable temperature of $900^{\circ} \mathrm{C}$ and drop the three cold capsules inside the hot oven. The comparison of the heating up rate of the fuel rod dummy thermocouples TC2 is shown in Figure 2.12.

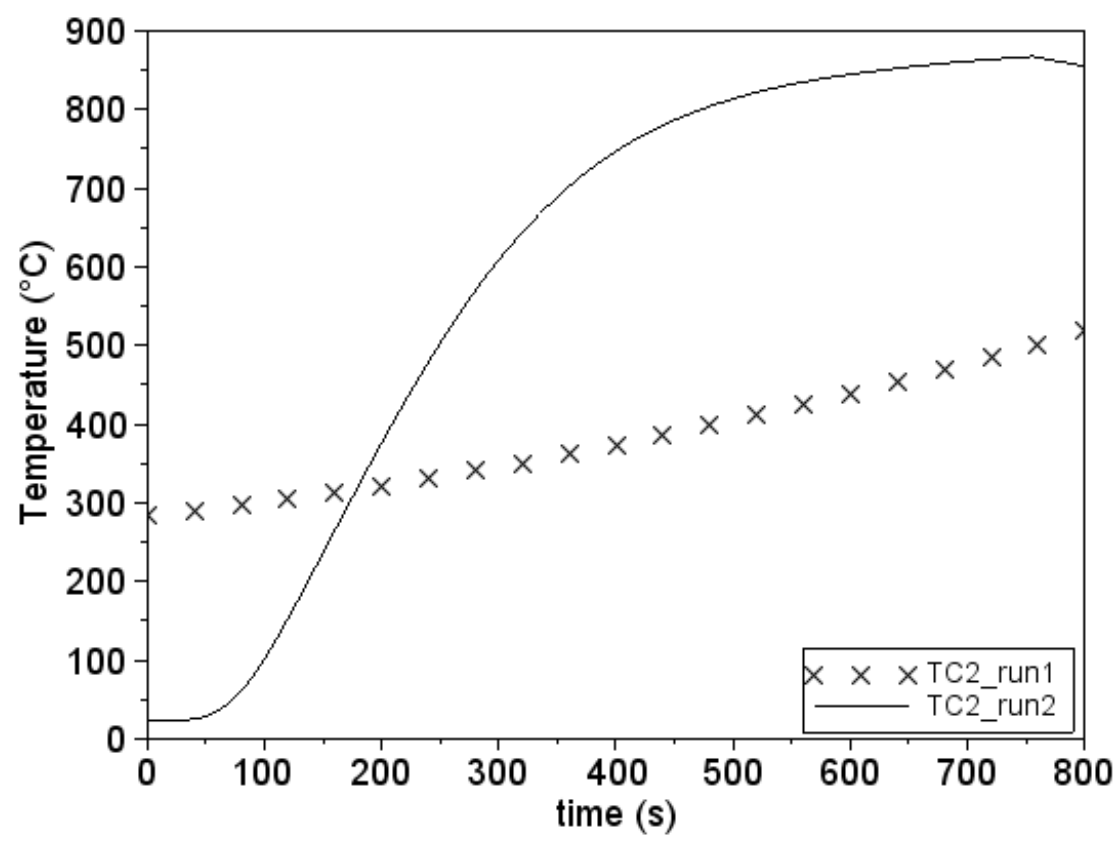

Figure 2.12: Comparison between commissioning run1 and run 2 Rod Thermocouple TC2

As it can be observed from the comparison, the heating up rate for Run 1 was smaller than the heating up rate for Run 2. Considering that the heating up rate is proportional to the difference of temperature between the oven and the capsule, with the run 2 scenario the heat flux to the capsule is larger because the oven is at the target temperature of $900^{\circ} \mathrm{C}$ when the cold capsules are dropped. Run 2 was selected as a preferred procedure and an additional test (Run 3 ) was performed to appraise the reproducibility of the heating scenario. The result of the reproducibility test is shown in Figure 2.13. Based on the plot of the figures and despite some potential variables that can perturb the heat transfer between the capsule and the rod (e.g. the capsule and rod surfaces have not been polished after the first 2 runs with a 
consequent difference in the emissivity of the two structures), the reproducibility of the test was considered satisfactory.

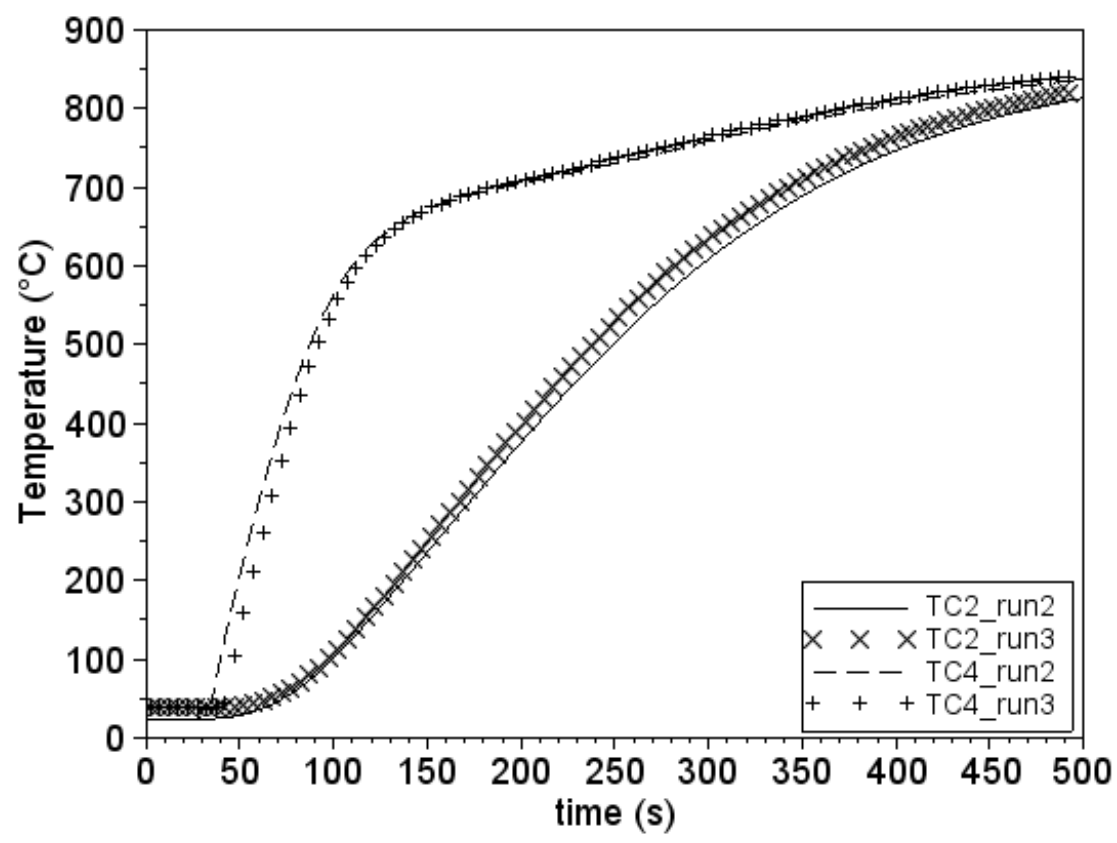

Figure 2.13: Comparison between commissioning run2 and run 3

Rod Thermocouple TC2; Capsule Thermocouple TC4

Run2 was selected as a reference case to complete the investigation of the first commissioning campaign. The configuration of the capsules on the same axial level raised the question whether the capsules are equally heated up and in particular if the azimuthal distribution of the temperature in the capsule and in the rod is uniform. The thermocouples arrangement of Figure 2.11 served to verify these two points. Figure 2.14 shows the recording of all the thermocouples during Run2.

The plot shows that TC1 and TC2 have an identical trend. This information is very important because it proved that the azimuthal distribution of temperature of the capsule is uiform. Concerning the other thermocouples, TC3 and TC4 had a similar trend and similarly TC5 and TC6. There was therefore a uniform azimuthal distribution of temperature in both the capsules and a very small difference of temperature between the two capsules. However, this delta becomes smaller when the two capsules approach $700^{\circ} \mathrm{C}$ and therefore before any relevant fuel events have occurred (e.g. rod ballooning, burst etc).

The last objective of the initial commissioning campaign was the quantification of the achievable heating up rate and its comparison with other LOCA tests. Table 2.1 shows the results of this investigation. 


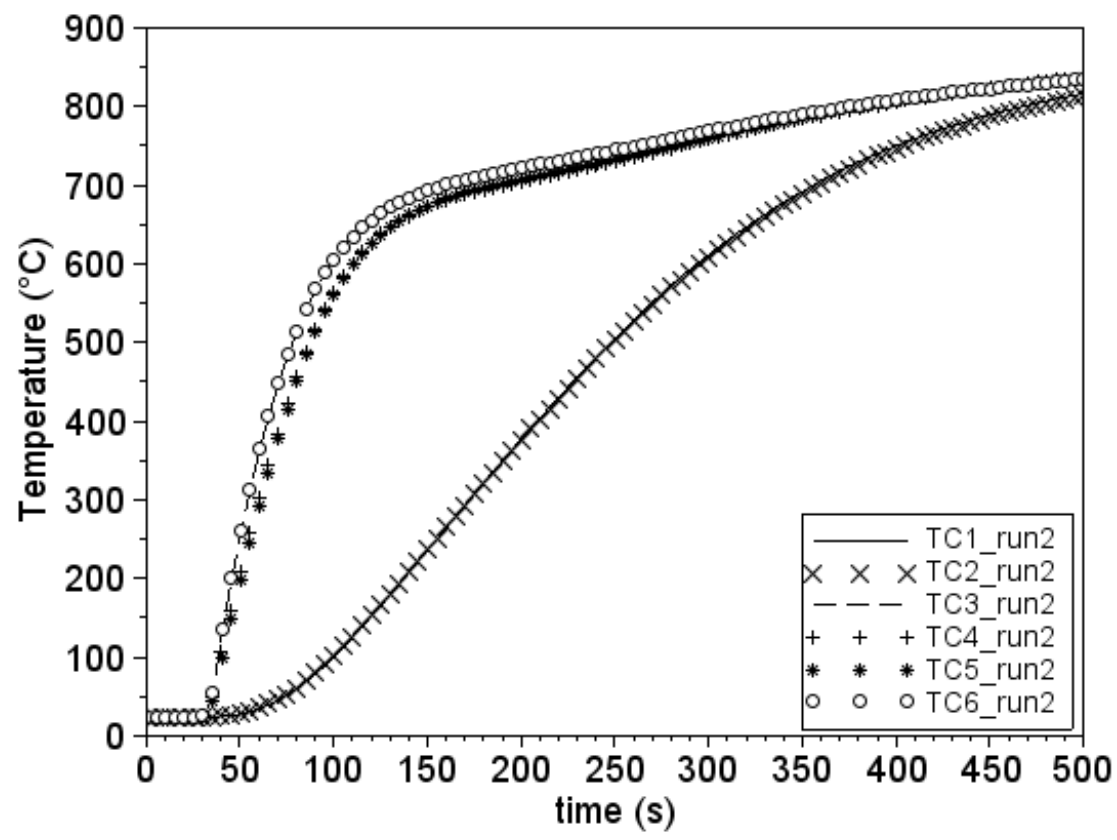

Figure 2.14: Recording of all thermocouple during commissioning Run 2 Rod Thermocouple TC1, TC2; Capsule Thermocouple TC3, TC4, TC5, TC6

\begin{tabular}{|l|c|}
\hline \multicolumn{1}{|c|}{ Test } & Heat up $\left({ }^{\circ} \mathrm{C} / \mathrm{s}\right)$ \\
\hline Run 2 & Circa 1.7 \\
\hline Halden Test IFA650-3 & 2.4 \\
\hline Halden Test IFA650-4 & 1.9 \\
\hline Halden Test IFA650-5 & 5.5 \\
\hline Halden Test IFA650-6 & 1.8 \\
\hline Halden Test IFA650-7 & 9.0 \\
\hline Halden Test IFA650-9 & 6.0 \\
\hline Halden Test IFA650-10 & 8.0 \\
\hline Halden Test IFA650-11 & 7.0 \\
\hline Halden Test IFA650-12 & 3.4 \\
\hline NRC Studsvik Test & 5 \\
\hline $\begin{array}{l}\text { FR-2- Karlsruhe }- \text { Institute } \\
\text { Technology }\end{array}$ & From 6 to 20 \\
\hline
\end{tabular}

Table 2.1: Comparison of heating up rate of different LOCA experimental program 
The average heating up rate is in the lower range with respect of other commissioning tests. In particular the value tends to decrease with the increase of the temperature of the cladding due to consequent smaller heat flux from the oven to the capsule and from the capsule to the rod. Fuel fragmentation has been observed in the literature despite differences in the heating up rate. Within the same order of heating up rate magnitude, the heating up rate does not seem to have a first order importance. For the purpose of the separate effects test, the heating up rate was considered satisfactory.

After the initial phase of commissioning trials with dummy capsules and rods, the detailed design of the capsule and rod to be used in the experiment started. This phase included also the selection of the rod design parameters to obtain the three design conditions discussed in 2.2.1. An illustration of the design of the rod and the capsule is provided in Figure 2.15.

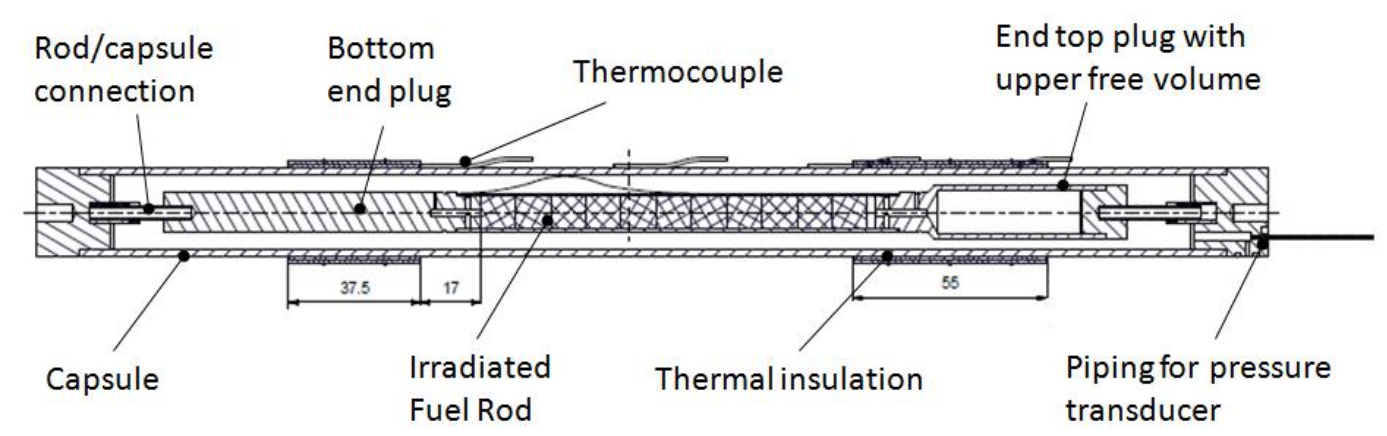

Figure 2.15: Details of the capsule and rod segment design

- Capsule: The capsule is made of ferritic steel and its diameter is $20 \mathrm{~mm}$. It should be underlined that the capsule has an important containment function in ensuring the retention of fission gases when the rod bursts. The structural integrity of the capsule was tested to prove that during the heating up its integrity function works as designed and withstands the pressure drop effect due to rod burst. The capsule was filled with Helium at 1 bar.

- Capsule thermocouples: Thermocouples were installed only on one of the capsules. The preliminary commissioning test shows that the capsules are subjected to similar heating up. Consequently, the temperature measurements of the installed thermocouples on one capsule are representative of the three capsules. No thermocouples have been installed on the cladding. During the preliminary commissioning test, where thermocouples were installed on the cladding (Figure 2.11), a thermo-dynamic model that calculates the heat transfer between the capsule and the cladding has been validated against the test results. This validated model has been used for all further calculations without need of further cladding temperature measurements.

- Pressure transducers piping: Each capsule is connected to a pressure transducer through small pipes. The pressure measurements of the capsules allow detecting the burst time. Moreover, the piping system allows the collection of gases at the end of the test. When Rod 2 bursts the gas inside the rod (initial fill in gas of the Helium and fission gas release during the transient) is 
accumulated in the capsule. The calculation of the difference between the initial gas content in the capsule and rod and the gas content at the end of the test is a measure of the fission gas release during the heating up.

- Fuel Rod: An irradiated fuel rod was used for the experiment (further details in 2.3). During the commissioning tests a rod consisting of Zircaloy cladding and alumina pellets was used.

- Bottom and top end plugs: The fuel rod has been manufactured and a bottom- and top- end plugs were welded to the extremities. The bottom end plug has only a supporting function, while the top end plug has also the function of free volume plenum that means that this volume is filled before the test with Helium. Each rod segment had a different plenum volume and fill-in gas pressure.

- The rod and the capsule are connected through a bar.

- Thermal insulation: A small thermal insulation was installed on the outer side of the capsule in correspondence to the axial location of the top- and bottom- end plugs of the rod to avoid a faster heating up of the rod's bottom and top-end plugs with a consequent axial heating flux from this structures to the ending of the rod and a generation of local hot spots. This measure was introduced following the experience gained during the commissioning campaign.

\subsubsection{Modeling of the Experimental Rig}

A thermo-dynamic model of the commissioning test was developed using ATHLET [4]. ATHLET (Analysis of Thermal-hydraulics of Leaks and Transients) is a thermal-hydraulic simulation code developed by the Gesellschaft für Anlagen- und Reaktorsicherheit (GRS) to study transient and LOCA condition in light water reactors. ATHLET was validated to be applied for several types of design basis and beyond design basis accidents. The full validation matrix can be found in the ATHLET models and methods [5].

The code was initially developed for light water applications. In the last decade, the code was upgraded and other working fluids have been included. This upgrade reflects the increased effort in the study of Generation IV nuclear reactor behavior [15] that considers the use as coolant of Helium [16], sodium [17], lead or lead-bismuth eutectic [18].

The possibility to use Helium as working fluid made ATHLET applicable to be used as simulation tool for the prediction of the thermal-hydraulic condition within the capsule. An illustration of the model developed in ATHLET is shown in Figure 2.16.

- The bottom part of the capsule is represented with the letter B1 and B2. In this area, the capsule made of steel surrounds the bottom end plug of the rod made of Zircaloy. Helium fills the gap between the two structures.

- The axial part of the capsule correspondent to the position of the fuel rod is divided in 10 axial nodes $(1,2,3,4 \ldots)$ from the bottom to the top of the fuel. The capsule is modeled as annular 
component made of steel while the fuel rod is modeled with two materials at different radial location: the fuel and the cladding with helium as a gap.

- The top part of the capsule is represented with the letter T. The capsule is modeled as an annular component made of steel. The top part of the rod is modeled as an annular component made of Zircaloy that represents the upper free volume structure that is filled in with helium. Helium fills also the gap between the capsule and the fuel rod top structure.

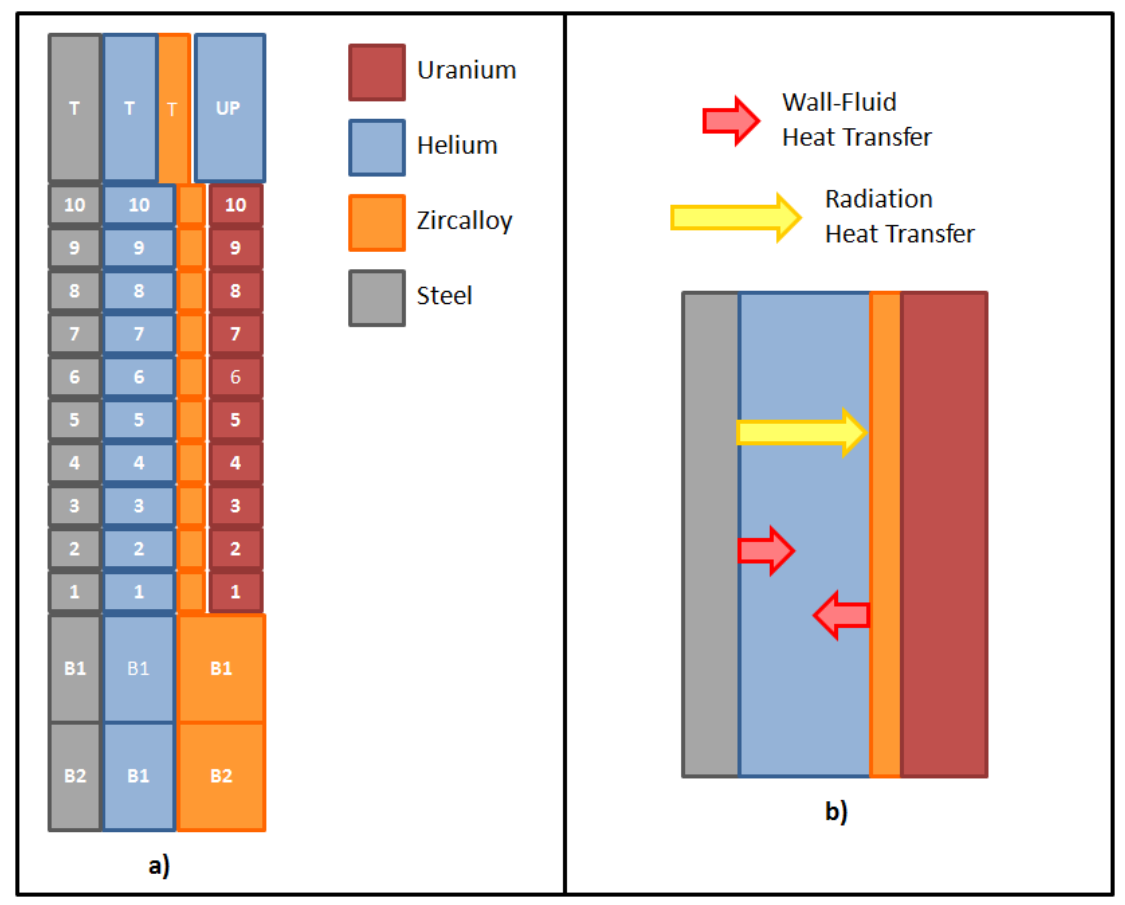

Figure 2.16: ATHLET Model of heat transfer between the capsule and rod segment a) Details of ATHLET nodalization; b) details of relevant heat transfer mechanisms

The boundary conditions used for the calculations are the measurements of the thermocouples on the outer part of the capsule at three different axial locations.

During the commissioning test, alumina pellets have been used to simulate the fuel. Alumina is not present in ATHLET as a pre-selected material. Consequently, a specified user's material has been built providing the code correlation of the temperature of the specific heat capacity, the thermal conductivity and the density taken from [19].

To perform the simulation with the irradiated fuel rod, the $\mathrm{UO}_{2}$ properties for high burnup fuel was considered. In particular ATHLET calculates the degradation of the heat conductivity as function of the fuel burnup [20].

The heat transfer regime within the capsule is governed by radiation and convection as illustrated in Figure 2.16 b). Considering the high temperature reached within the loop, also the radiation heat 
transfer coefficient is playing an important role. In ATHLET the emissive surfaces are gray, so that spectral coefficients of emissivity follow the Lambert Law. Bodies are treated as infinitely long cylinder bodies. In ATHLET, calculations are performed inside a heat conduction volume, independently of neighboring volumes and the radiative heat transfer is taken into account only for flow regime with rather high void fraction in the core channel (not relevant in the calculation performed with Helium as fluid).

For the calculation, the selection of the view factors was calculated based on the instruction of Figure 2.17.

\begin{tabular}{|c|c|c|}
\hline $\begin{array}{c}\text { Between concentric } \\
\text { infinite cylinders of radii } \\
R_{1} \text { and } R_{2}>R_{1} \text {, with } \\
r=R_{1} / R_{2}<1\end{array}$ & $\begin{array}{c}F_{12}=1 \\
F_{21}=r \\
F_{22}=1-r\end{array}$ \\
\hline
\end{tabular}

Figure 2.17: View factor of concentric infinite cylinders

Since the capsule has been used for different commissioning tests and it was subject to polishing after the test, a value of emissivity for polished steel surfaces of 0.7 has been used. An important parameter to investigate is the cladding material emissivity. MATPRO/RELAP5-3D [21] contains a library of material properties for light water reactor accident analysis. Figure 2.18 was extracted from [21] and it summarizes the plausible values of cladding emissivity.

The model developed calculates the axial temperature distribution of the cladding, of the bottom and top structures and the thermodynamic properties of the capsule environment. This data were used as input for the TRANSURANUS calculation to appraise the fuel rod properties during the transient and calculate particularly the occurrence of burst and cladding deformation.

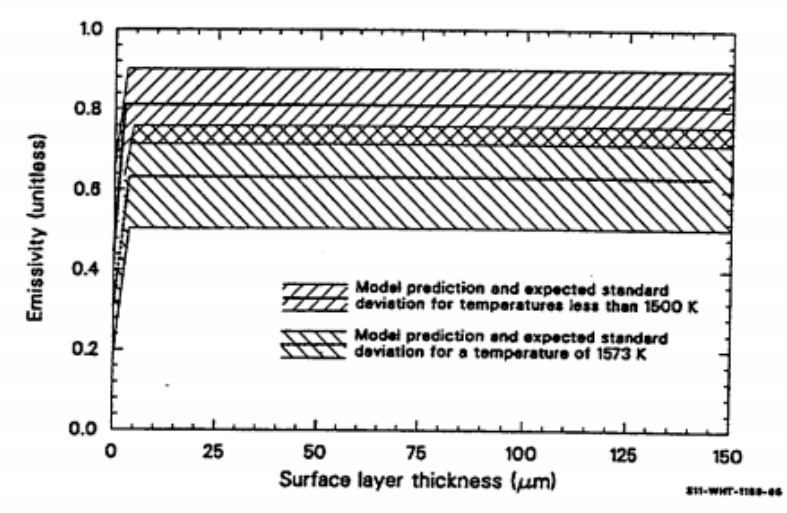

Figure 2.18: Value of emissivity for fresh and irradiated cladding Figure from Reference [21] 


\subsubsection{ATHLET-TRANSURANUS Methodology Validation}

The methodology developed to calculate the properties of the fuel rod during the separate effects test needed to be first validated against already available experimental data. In this section, the ATHLETTRANSURANUS methodology is described and a complete validation against IFA-650.13 is shown. Additionally, at the end of the section, key results are presented also for IFA-650-2 and IFA-650.3.

IFA-650.13 was performed on the $22^{\text {nd }}$ of October 2012. The fuel rod used is a BWR fuel rod irradiated in Leibstadt NPP up to a burnup of $72 \mathrm{MWd} / \mathrm{kgU}$ [23]. As a consequence of the LOCA transient, the fuel cladding experienced ballooning and burst. The target PCT was $850^{\circ} \mathrm{C}$.

The methodology used to perform the calculation is based on the following steps:

a) An ATHLET model has been built (Figure 2.19) based on the geometrical data of the experimental rig inside the reactor (where the fuel rod segment is located) and part of the external loop including the blow down tank.

b) The thermal-hydraulics boundary conditions were extracted from ATHLET and a transient input file for TRANSURANUS was built with the following ATHLET data during the transient:

b.1 Test rig pressure

b.2 Fuel rod Average Linear Heat Generation Rate (ALHGR)

b.3 Outer fuel cladding temperature axial distribution

b.4 Test rig lower plenum temperature and pressure

b.5 Rod upper free volume temperature

c) A TRANSURANUS model was built with the rod information and considering the rod power irradiation history [23]. A calculation was performed to "irradiate" the fuel rod and to change the properties of the fuel and pellet to simulate a high burnup fuel segment of $72 \mathrm{MWd} / \mathrm{kgU}$.

d) The fuel rod was "remanufactured". The restart file containing the fuel rod information at the end of the irradiation from point $\mathrm{c}$ ) has been modified to simulate the manufacturing of the rod segment at the Halden laboratory. The parameter to change was the fuel rod internal pressure. In fact, after the irradiation in the commercial reactor, the inner rod pressure was the result of the initial rod fill-in pressure and the build-up of the fission gas release during the irradiation. The rod remanufacturing performed in the hot cells of HRP in Kjeller consisted of welding the irradiated fuel segment to a bottom and upper end structures. The upper structure contained an upper free volume that was filled with gas at room temperature to reach a pressure of 20 bar. Therefore, in the restart file, a modification was applied so that before the LOCA transient, the rod upper free volume had a pressure of 20 bar at room temperature. It should be underlined that when the main irradiated rod was cut in the hot cell before to be used for remanufacturing of the segments, the FGR during irradiation is released in the hot cell environment.

e) The modified restart file obtained in the step d) together with the ATHLET boundary conditions of step b) were used to perform the TRANSURANUS LOCA transient. 
The ATHLET version used for the simulation is the latest version released by GRS: ATHLET Mod 3.0 Cycle A [20].

The ATHLET model has as boundary condition the loop inlet valve VA6304 and the loop outlet valve 6305. The blow down line up with the "LOCA" valve VA6333 up to the blow down tank 6301 was entirely modeled. Appropriate boundary conditions assigned to the ATHLET end junctions representing VA6304 and VA6305 can determine the operation of the loop prior to the LOCA test.

The different colors of the pipes in Figure 2.19 correspond to different external temperature boundary conditions for the piping system. PIPE-DIS, PIPE-IN and PIPE-OUT are external to the reactor lid. The pipes RIG-IN and RIG-OUT are inside the reactor lid. To simulate the normal operation of the loop, constant inlet mass flow is provided at the end-left of PIPE-IN; the flow goes through PIPE-IN, RIG-IN, PIPE-CH (that represents the thermal-hydraulic channel where the fuel rod segment is located) and then the mass flow goes out the rig through RIG-OUT, PIPE-OUT. At the right-end of PIPE-OUT the component PRES-CTR is the right boundary condition for the loop. It should be underlined that ATHLET components are one-dimensional components, therefore, the $y$-axis properly represents the elevation of the different components, but the $x$-axis represents the horizontal length only from a representative point of view, because the 2-D dimension is not considered in the formulas resolution.

Figure 2.20 shows details of the nodalization of the experimental rig. The mass flow enters at the bottom of the experimental rig (RIG-IN) and then it flows through the two annular channels. The first annular channel is the channel between the fuel rod and the heater (PIPE-CH), and the second channel is the channel between the heater and the flask (PIPE-HEAT). The two annular channels communicate with each other at the bottom and top through small holes. The mass flow goes out the experimental rig through the outlet flow tube (RIG-OUT) connected to PIPE-HEAT. In the previous HALDEN LOCA test experiment IFA-650, a spray has been used during the LOCA transient to provide enough steam to the cladding for the oxidation and to control the PCT (SPRAY). In the IFA-650.13 test the spray was not used.

At the beginning of the transient, critical flow is achieved on the discharge line and the critical flow has been modeled with the non-thermal equilibrium model developed by GRS CDR1D [5]. Following the first phase of the blow down, the model makes an automatic transition to the regular solving of the momentum formula at the relevant junction. The relevant heat transfer mechanisms within the IFA-650 test rig are shown in Figure 2.21.

The nodes of the fuel rod and heater with numbers are the nodes that actively generate power. It should be underlined that the heater is slightly longer $(581 \mathrm{~mm})$ than the fuel rod $(380 \mathrm{~mm})$. The active zone of the fuel is divided in 10 axial equally spaced nodes. There is a similar axial nodalization between the fuel rod, the heater and the flask to allow a proper evaluation of the radiation heat transfer.

Due to the increase of temperature of the fuel rod during the transient and the temperature difference between the cladding and the heater and the heater and the flask, the radiation heat transfer plays an important role. In 2.2.3 information on the modeling in ATHLET of the radiation heat transfer were provided. For the value of the emissivity it was estimated to be about 0.85 (Figure 2.18) considering an oxide thickness of $20 \mu \mathrm{m}$ taken from [23]. The flask material is stainless steel 316. 


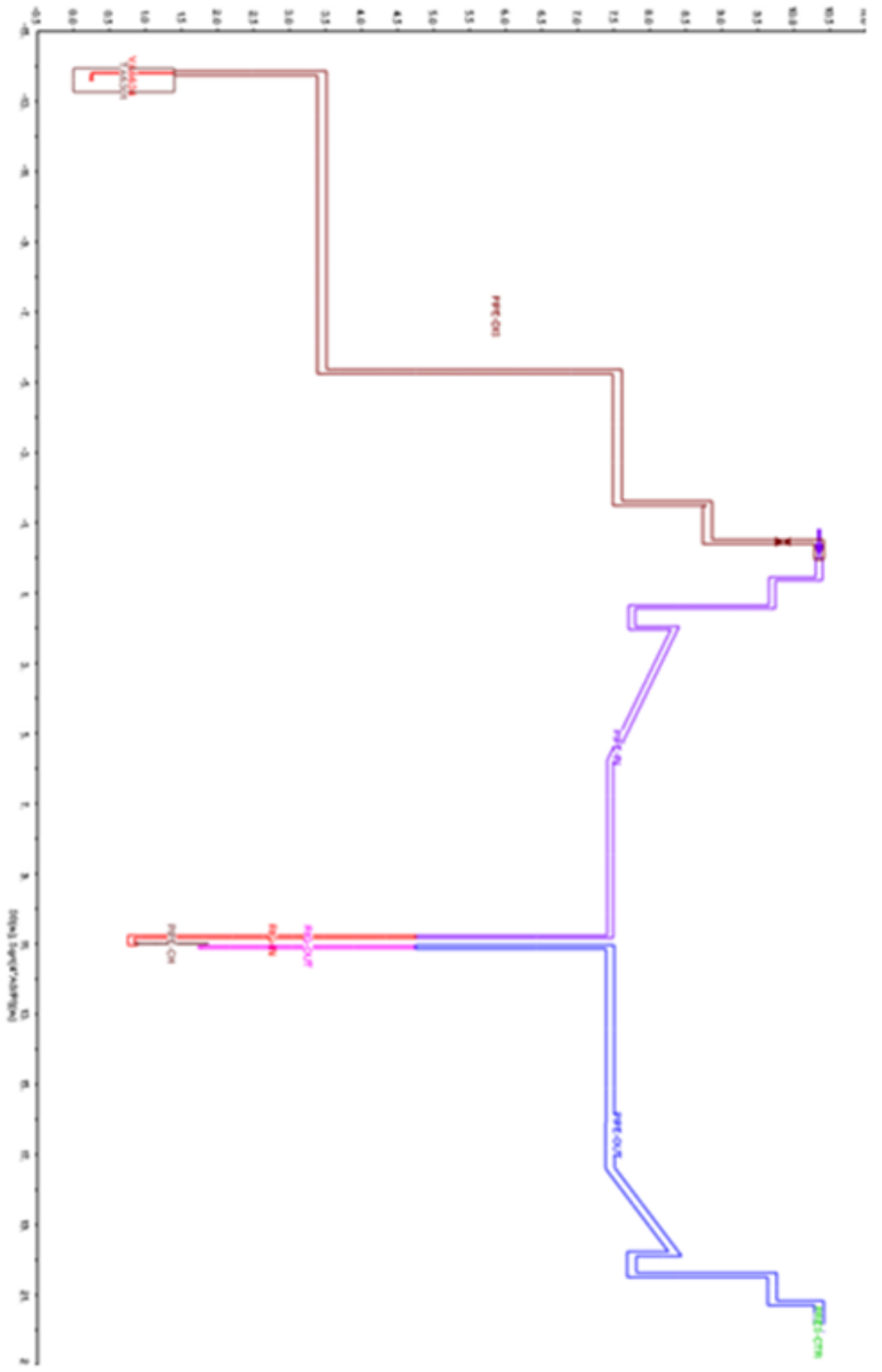

Figure 2.19: Details of ATHLET nodalization of IFA-650 loop 


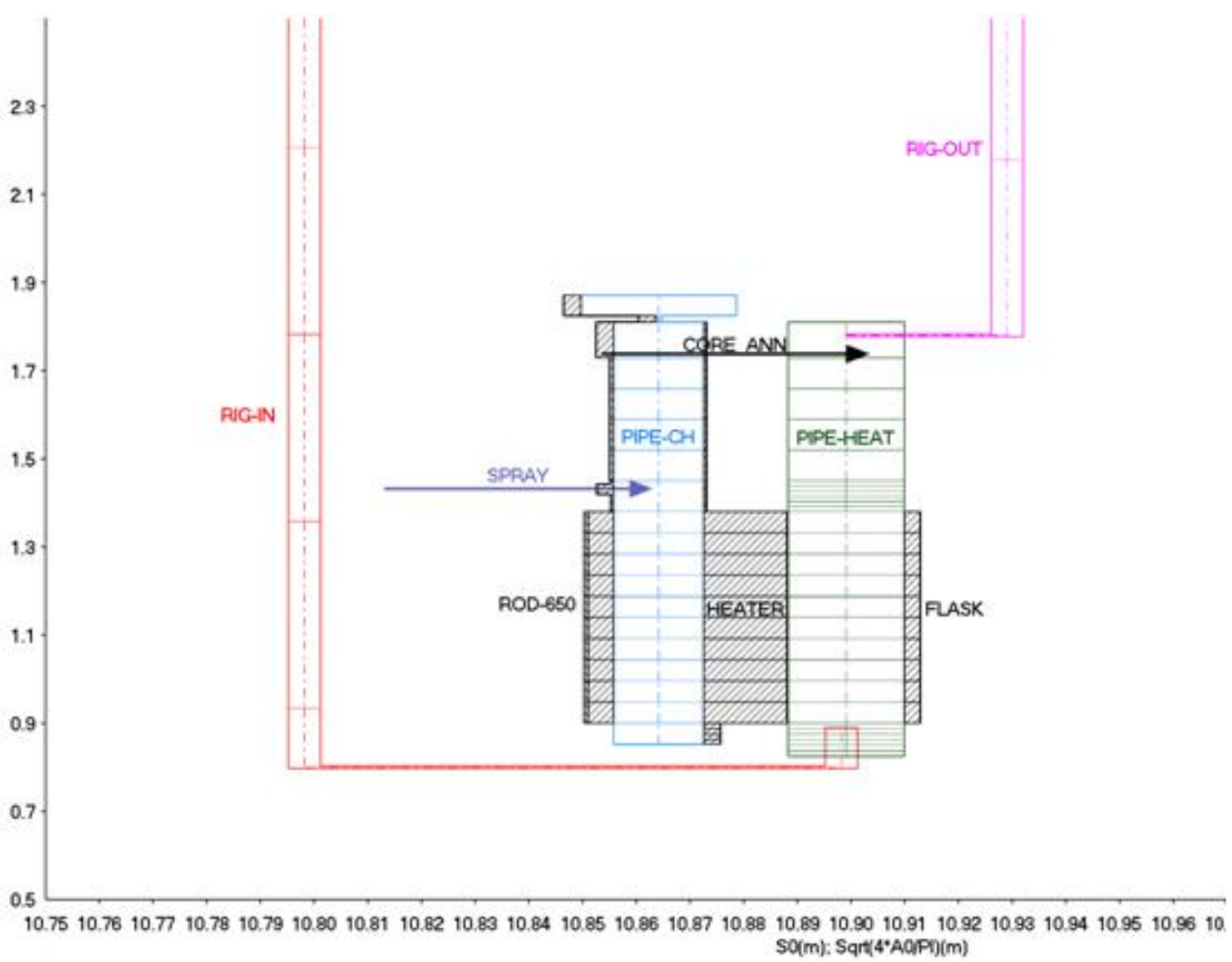

Figure 2.20: Details of the ATHLET nodalization of IFA-650 test rig

\begin{tabular}{|c|c|c|c|c|c|}
\hline Fuel Rod & & Heater & & Flask & \multirow{3}{*}{$\Rightarrow \begin{array}{l}\text { Wall-Fluid } \\
\text { Heat Transfer }\end{array}$} \\
\hline & & 12 & & & \\
\hline 10 & & 11 & & & \\
\hline 9 & $\Rightarrow$ & 10 & $\Rightarrow$ & & \multirow{10}{*}{$\underset{\begin{array}{l}\text { Radiation } \\
\text { Heat Transfer }\end{array}}{\stackrel{2}{\Rightarrow}}$} \\
\hline 8 & 4 & 9 & $\Leftrightarrow$ & & \\
\hline 7 & & 8 & & & \\
\hline 6 & & 7 & & & \\
\hline 5 & $\Leftrightarrow$ & 6 & $\Leftrightarrow$ & & \\
\hline 4 & & 5 & & & \\
\hline 3 & & 4 & & & \\
\hline 2 & & 3 & & & \\
\hline \multirow[t]{2}{*}{1} & & 2 & & & \\
\hline & $\pi$ & 1 & 介 & & \\
\hline
\end{tabular}

Figure 2.21: Heat transfer mechanisms in IFA-650 
Two TRANSURANUS calculations were run with the code version V1M1J11 [6]: 1) The irradiation calculation to simulate the irradiation in the commercial reactor and 2) the LOCA transient based on the ATHLET thermal-hydraulics boundary conditions. The TRANSURANUS model is shown in Figure 2.22.

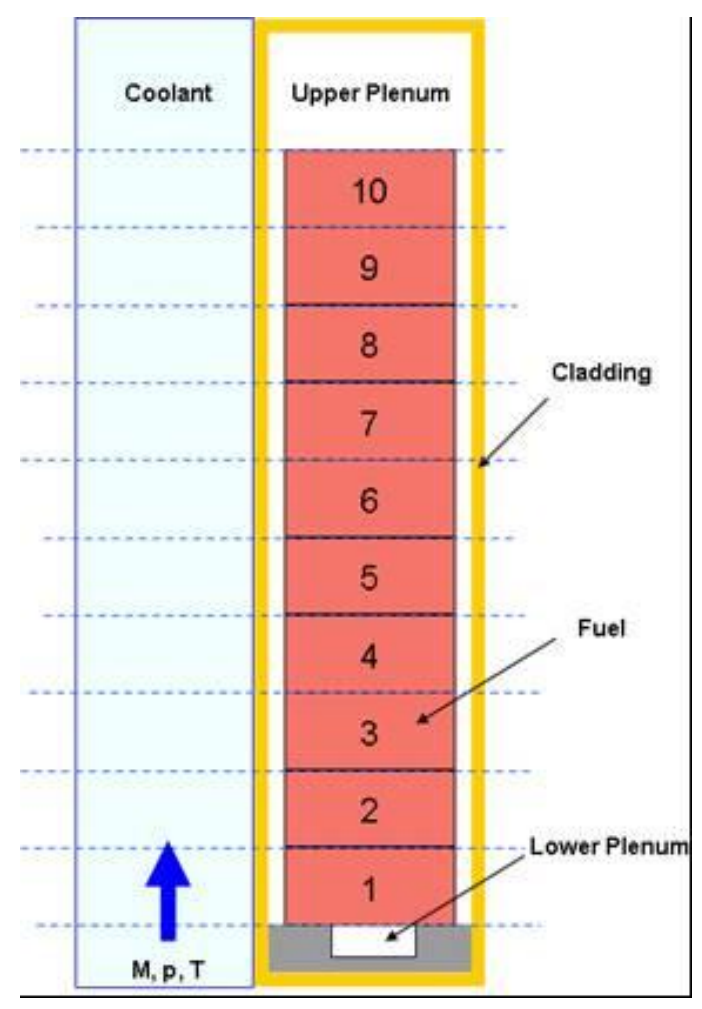

Figure 2.22: TRANSURANUS model for irradiation and LOCA calculations

The fuel rod is divided in 10 equal axial nodes of the length of $38 \mathrm{~mm}$. The upper plenum has a length of about $287 \mathrm{~mm}$. The axial length $(\mathrm{L})$ of the upper plenum has been determined considering that the upper free volume $(\mathrm{V})$ is between 15 and $16 \mathrm{cc}$, where $r$ is the inner cladding radius.

$$
L=\frac{V}{\pi \cdot r^{2}} \quad \text { (Formula 2.1) }
$$

The fuel rod power history is shown in Figure 2.23 (segment E) [23].

During the irradiation phase, as boundary condition for the coolant, values for a generic BWR have been considered:

$$
\left\{\begin{array}{c}
p=73 \mathrm{bar} \\
T=288^{\circ} \mathrm{C} \\
\dot{m}=1.57 \cdot 10^{6} \frac{g}{h} \quad \text { (Formula 2.2) }
\end{array}\right.
$$

The linear heat generation rate has been extracted from Figure 2.23 (segment E). 


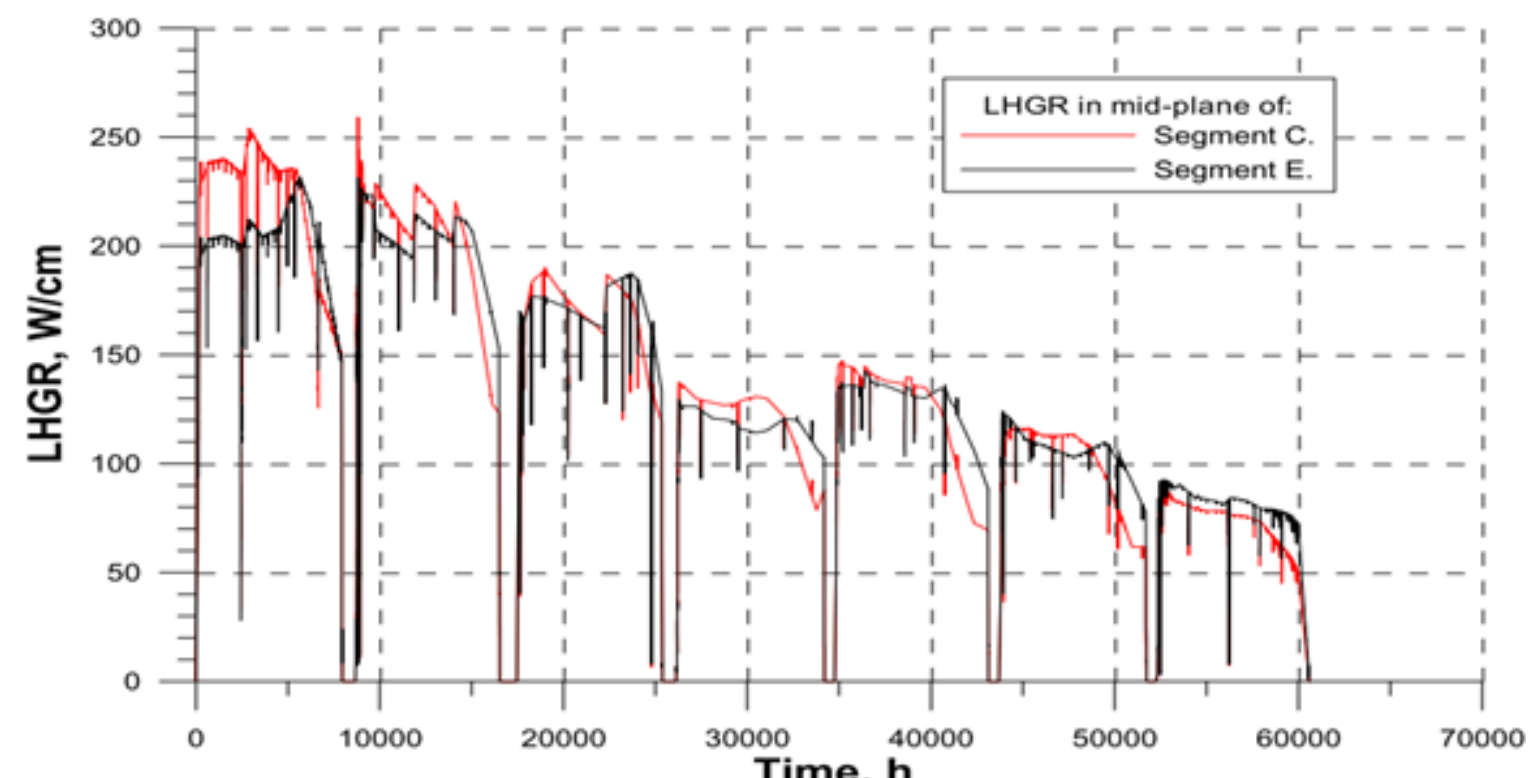

Figure 2.23: IFA-650.13 power history

Figure from Reference [23]

The important options and models used in TRANSURANUS are listed below. Additional information can be found in the TRANSURANUS User Manual [6]:

- URGAS Model for fission gas release: The URGAS model is based on an effective diffusion process. The model can solve the diffusion equation and therefore the fission gas release for time varying the power history (e.g. gas production rates and temperatures) but also if the power keeps constant, the model takes into account the variation of the other properties of the fuel (gap conductance, radial power distribution within the pellet) [26].

- High Burnup Structure model as described in 2.1.1.

- Cladding corrosion during irradiation calculated based on the MATRPO model for BWR condition [21] as shown in Figure 2.24.

- The oxidation model selected during LOCA ( $T>673 \mathrm{~K}$ ) is the Leistikow steam oxidation model. For temperature below the threshold the MATPRO model applies.

- The burst criteria considered in the calculations are the following two [27]:

- The first cladding failure criterion is based on stress assessment, that is, the comparison of the calculated tangential stress with a distinct failure threshold (overstress criterion). The failure threshold of the cladding alloy is defined as a function of the actual temperature and oxidation level in the material property function.

- The second failure principle is a simple "plastic instability criterion" based on the simultaneous assessment of the effective true strain and the strain rate. When both the strain and the strain rate exceed the threshold values of 0.025 and $1001 / \mathrm{h}$, respectively (default values), the cladding is assumed to be ruptured. These parameters are introduced in the model as input parameters. This criterion is applied concurrently to an 
"overstrain criterion" (third out of three criteria) that is, if plastic instability is not indicated, then the overstrain criterion is checked automatically. This criterion limits the true tangential plastic strain to the maximum of $50 \%$. These criteria were introduced particularly for LOCA analyses, due to the significant uncertainty of the stress computation at large cladding deformation.

- As described above, the upper free volume pressure has been changed after the irradiation calculation and before running the transient so that the fill in pressure obtained in Halden during re-fabrication can be considered. To find the amount of moles needed in the upper free volume to achieve the pressure of 20 bar at Room Temperature (RT), the ideal gas law can be used:

$$
\begin{aligned}
& p=20 \mathrm{~atm} ; \\
& V=15.5 \mathrm{cc}=0.0155 \mathrm{~L} \\
& T=20^{\circ} \mathrm{C}=293.15 \mathrm{~K} \\
& R=0.08206 \frac{\mathrm{L} \cdot \mathrm{atm}}{\mathrm{mol} \cdot \mathrm{K}} \\
& P \cdot V=n \cdot R \cdot T ; \quad n=\frac{\mathrm{p} \cdot \mathrm{V}}{R \cdot T}=\frac{20 \cdot 0.0155}{293.15 \cdot 0.08206}=12887 \mathrm{\mu mol}
\end{aligned}
$$

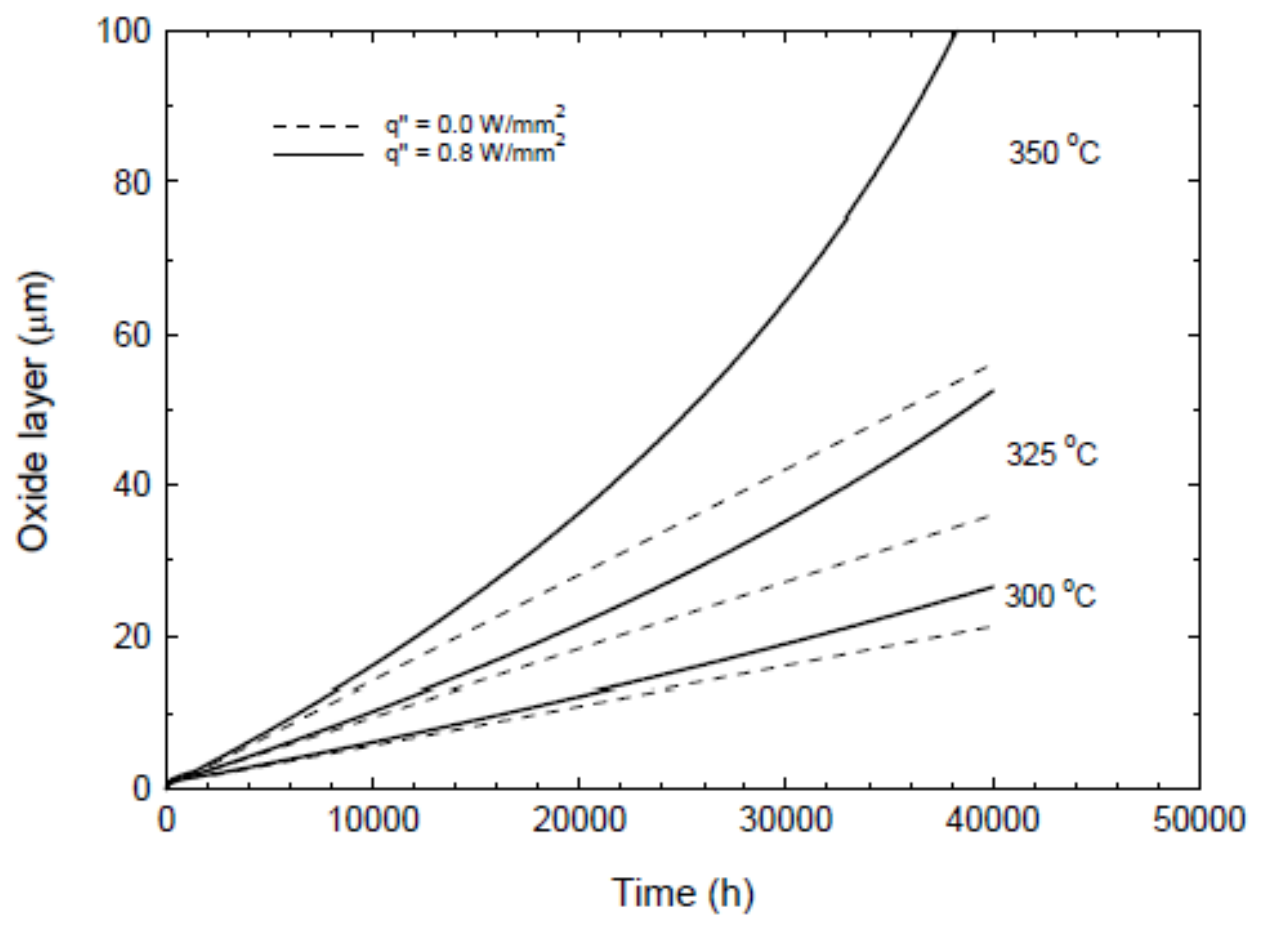

Figure 2.24: MATPRO corrosion correlation for BWR conditions

The results of the calculations and the benchmark with the data recorded during the experiment are shown in Figure 2.25-2.28. 
As it can be observed from the figures, the ATHLET calculation is able to predict with good accuracy the LOCA test. The blow down of Figure 2.25 presents only a very small discrepancy for value of pressure below 30 bar. However, the ATHLET calculation predicts very well the time at which the depressurization is completed (after about 120 seconds). The inlet and outlet thermocouples shown in Figure 2.25 are also qualitatively and quantitatively well reproduced. The small discrepancies can be associated with the nodalization of the upper and lower plenum of the test rig as a branch component. The cladding and heater thermocouples (Figure 2.26) are well reproduced. There is a discrepancy after about 250 seconds, in which the calculation over-predicts the cladding temperature. Observing Figure 2.26, it can be seen that the discrepancy starts at about 250 seconds. The over prediction seems to be due to the ballooning that starts at about 250 seconds (see Figure 2.28). When the cladding balloons, the heat resistance in the fuel/cladding gap increases and there is a change in the radial heat transfer within the fuel. ATHLET considers fixed geometries and therefore this phenomenon is not taken into account.

Figure 2.28 shows the TRANSURANUS calculation of the evolution of the pressure within the fuel rod and the final cladding axial deformation. The rod inner pressure initially decreases following the reduction of temperature of the rig due to the increase in the heat transfer coefficient during the flashing phase. Then the pressure starts to increase following the ramping up of the cladding temperature. It should be underlined that the pressure within the rod does not increase proportionally to the increase of temperature of the cladding, because part of the upper free volume is on top of the fuel in an unheated area. The ballooning starts when the pressure reaches its maximum (in the experiment at about $250 \mathrm{~s}$ ). The progression of the ballooning and clad deformation can be detected by the reduction of the inner rod pressure in a phase in which the cladding temperature is still increasing. The deformation proceeds until the burst criterion is met: at $300 \mathrm{~s}$ based on the experimental data and $308 \mathrm{~s}$ based on the TRANSURANUS calculation.

The TRANSURANUS prediction of ballooning and burst is satisfactory. From the TRANSURANUS calculation it is possible to extract also the axial cladding deformation at the time of the burst and this is also shown in Figure 2.28. The axial node that meets the burst criterion is number 7 (axial position between 228 and $266 \mathrm{~mm}$ ).

As additional validation, similar calculations have been performed for IFA-650.2 and IFA-650.3 and the results are shown in Figures 2.29 and 2.30 further confirming the ATHLET-TRANSURANUS model used later on to design the experiment. 

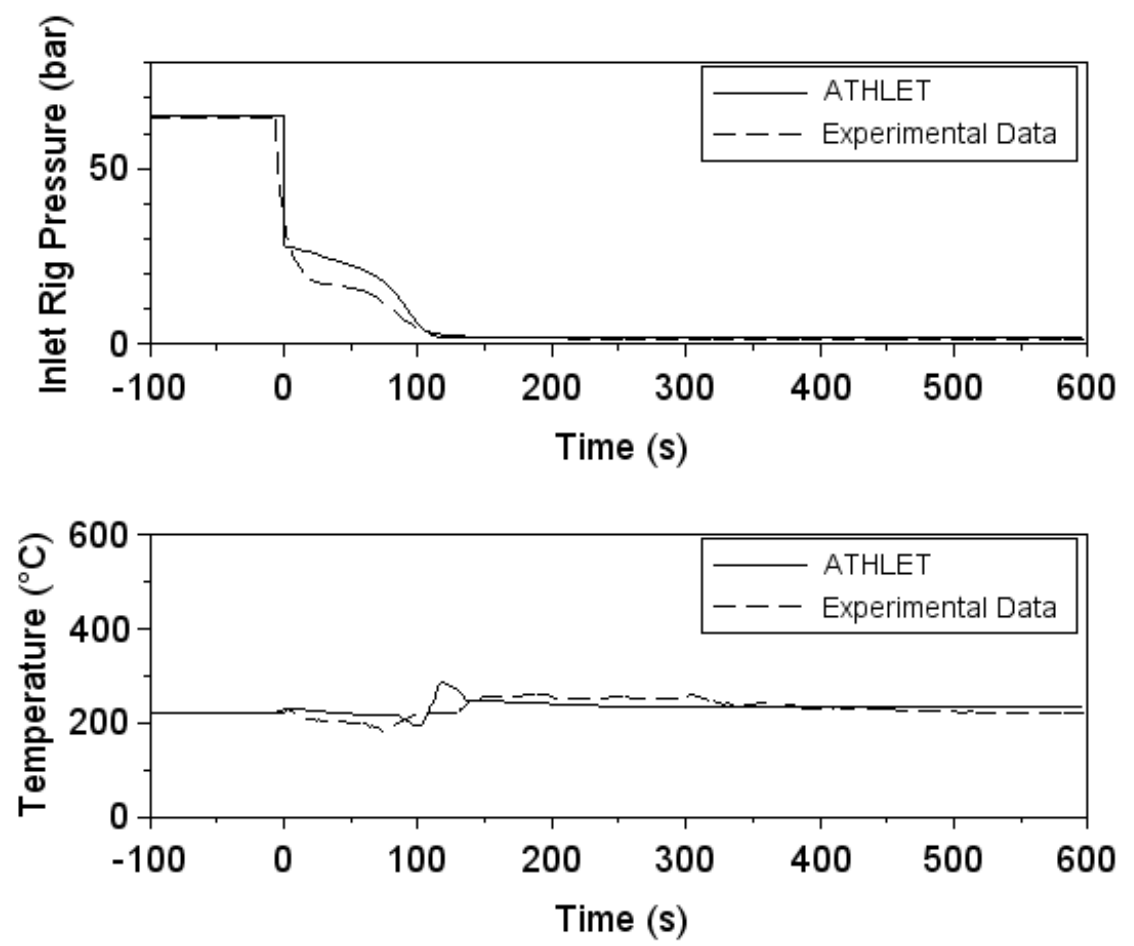

Figure 2.25: ATHLET IFA-650.13 rig pressure and inlet temperature Figure from Reference [23]
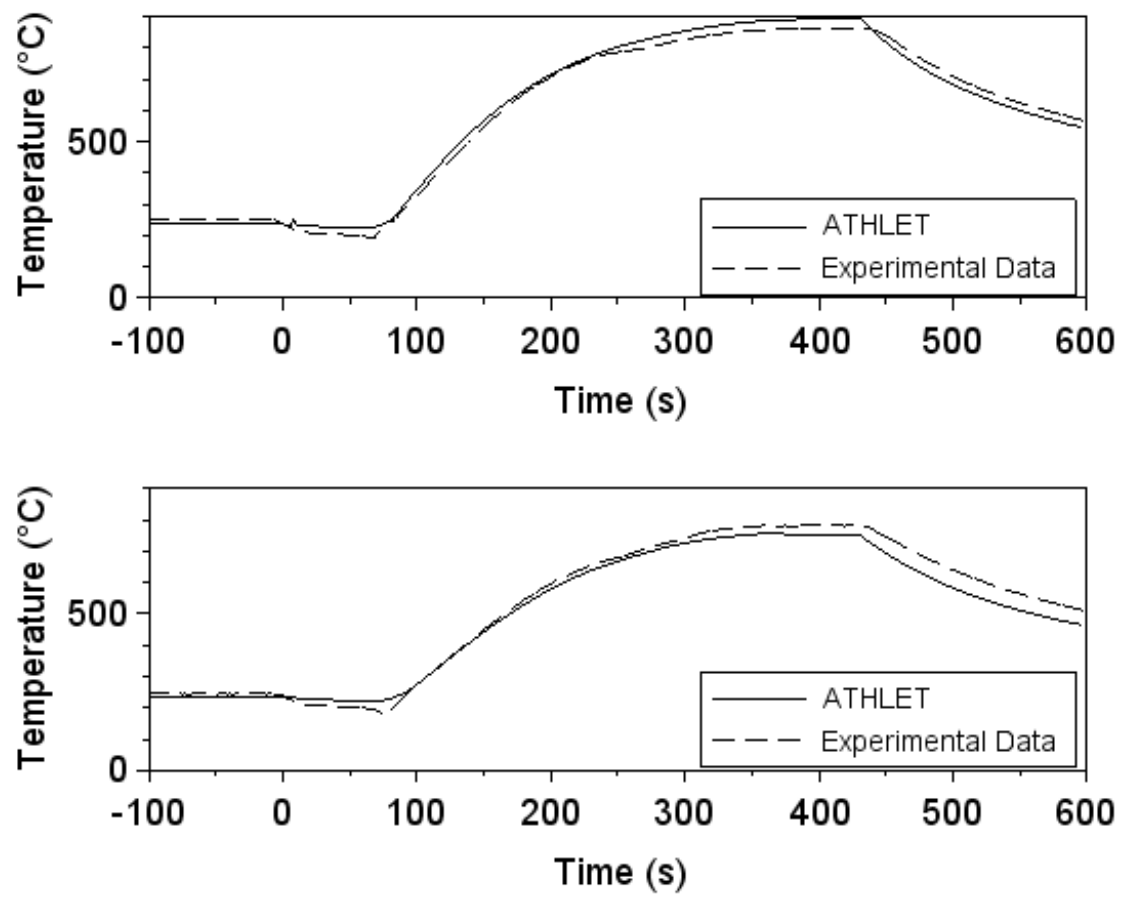

Figure 2.26: ATHLET IFA-650.13 temperatures transient Top Figure: Cladding Bottom Thermocouple; Bottom Figure: Heater Bottom Thermocouple; Data as from Reference [23] 

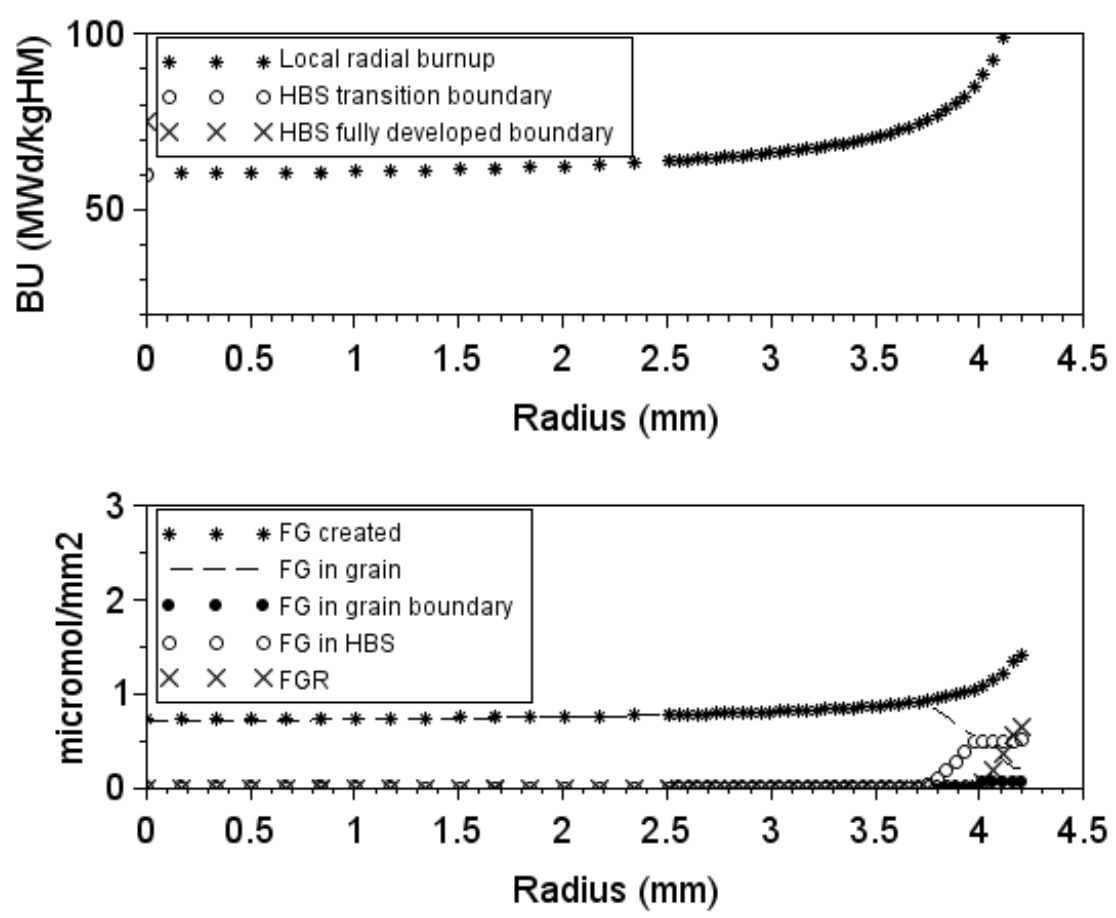

Figure 2.27: TRANSURANUS IFA-650.13 end of irradiation radial burnup and fission gases Data as from Reference [23]
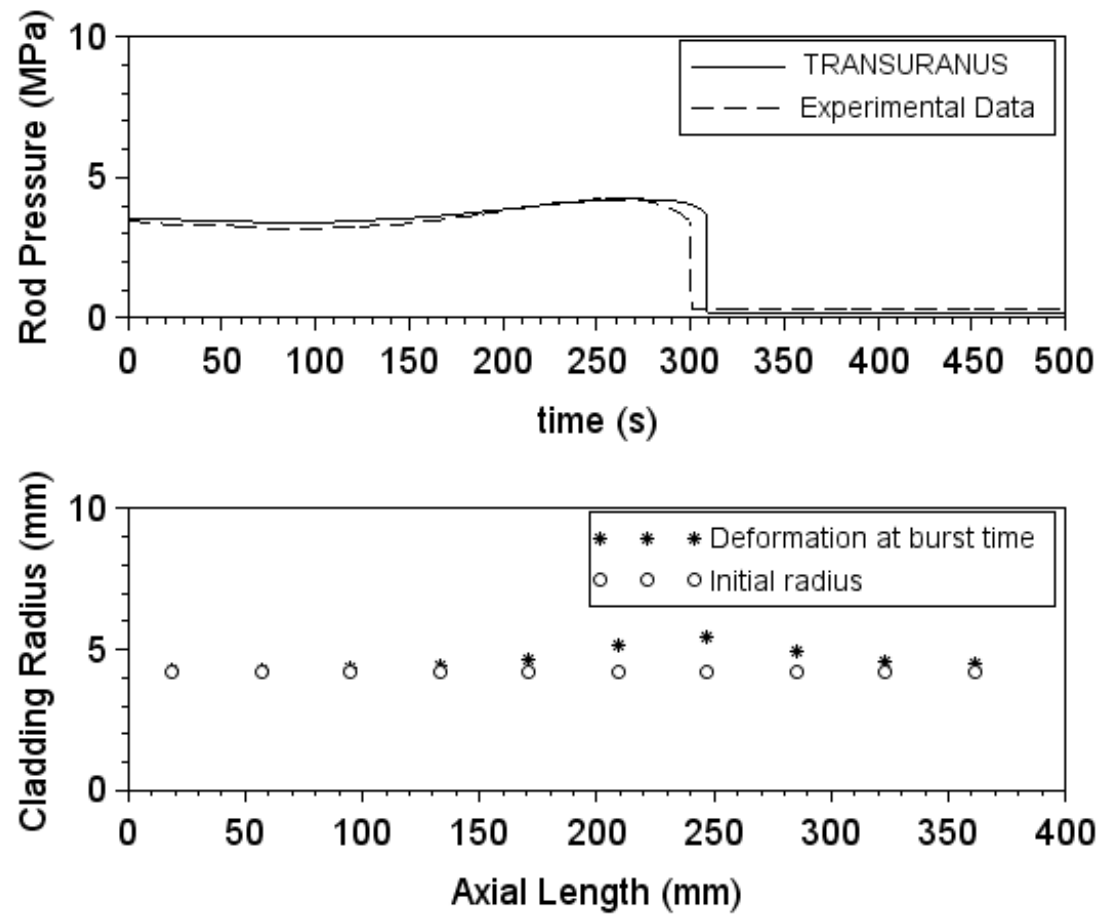

Figure 2.28: TRANSURANUS IFA-650.13 LOCA rod internal pressure and cladding deformation Data as from Reference [23] 

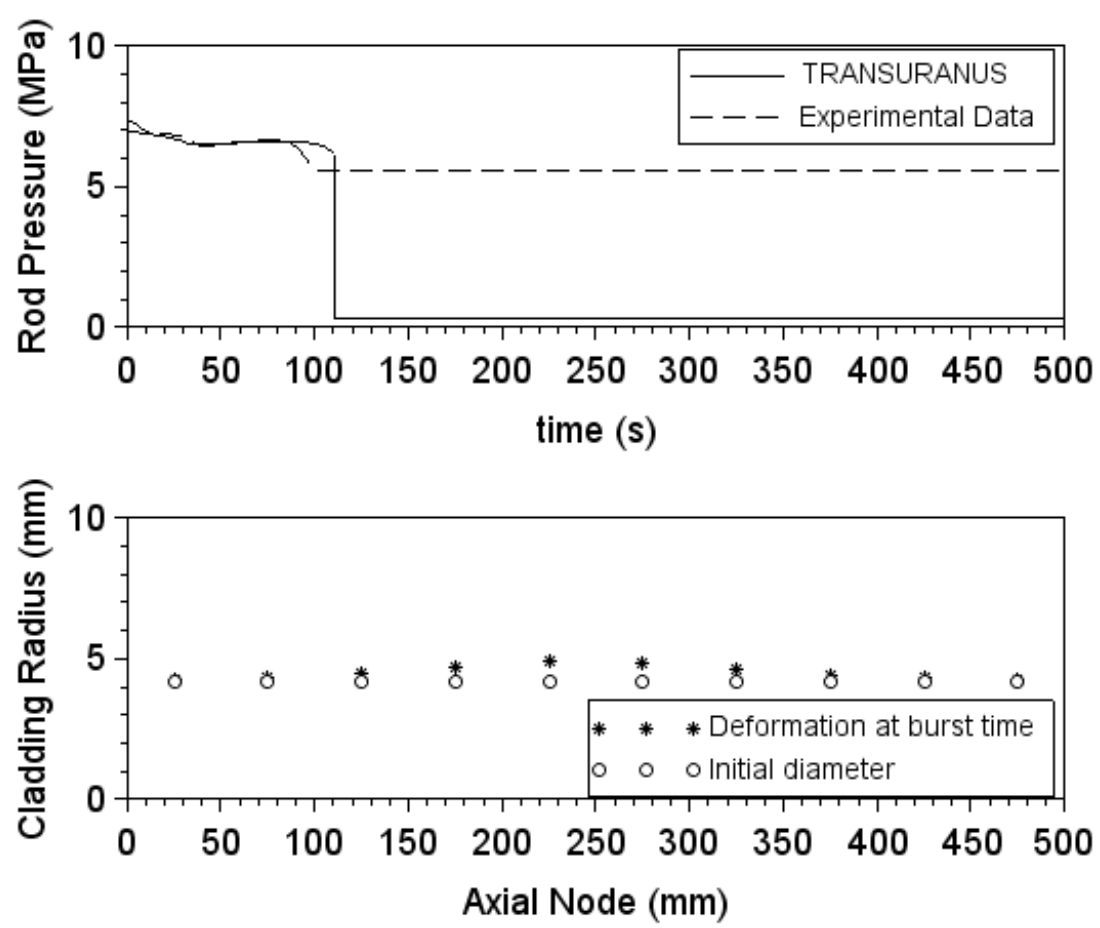

Figure 2.29: TRANSURANUS IFA-650.2 LOCA rod internal pressure and cladding deformation
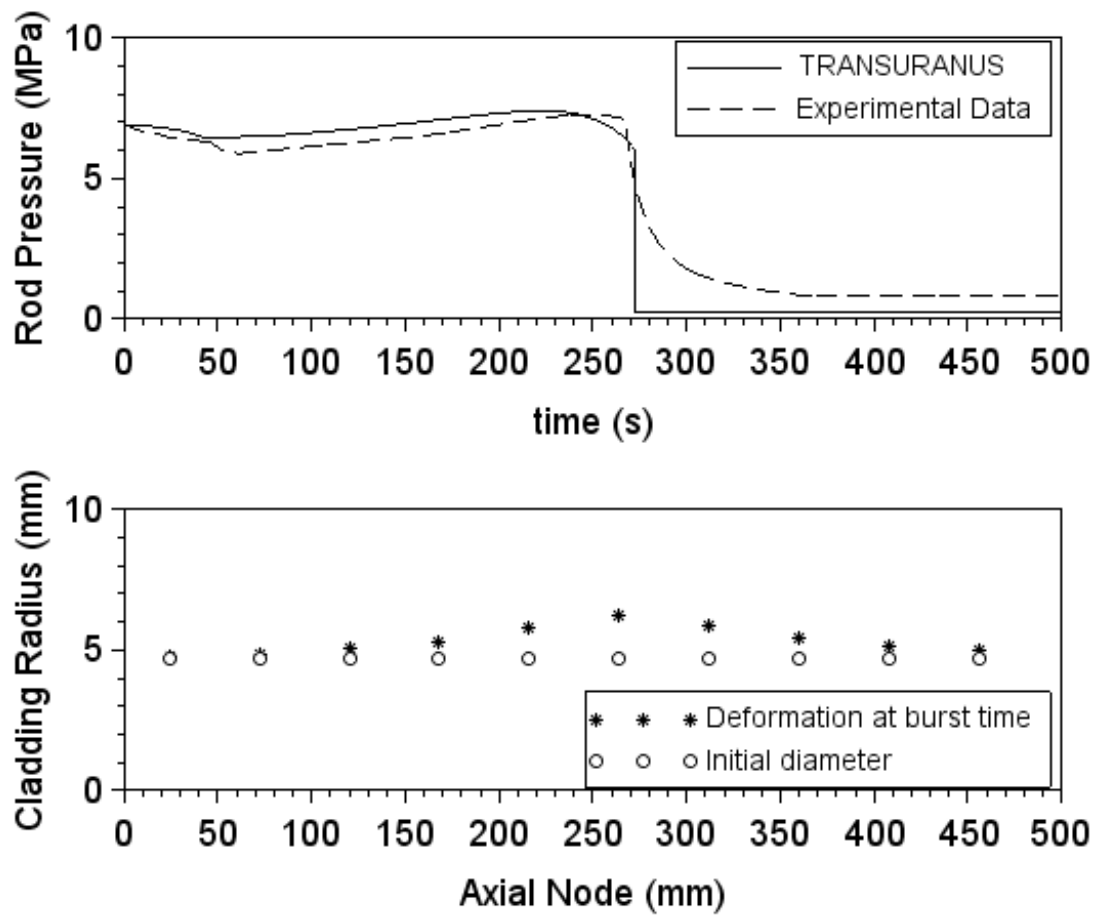

Figure 2.30: TRANSURANUS IFA-650.3 LOCA rod internal pressure and cladding deformation 


\subsection{Fuel rod segment behavior}

The design methodology based on ATHLET-TRANSURANUS behavior against the IFA-650 test as described in the previous chapter was used to simulate the transient of the three rod segments with the purpose of selecting appropriate fuel rod design parameters to obtain the boundary conditions discussed in 2.2.1 The selection of the fuel rod design parameters was a prerequisite for proceeding with the remanufacturing of the irradiated fuel segments.

The information about the irradiated fuel segment is given in 2.3.1 together with an evaluation of its properties after irradiation based on the calculation with TRANSURANUS. In 2.3.2 a TRANSURANUS transient evaluation was performed in order to predict the cladding deformation and burst of the rod based on selected fuel rod design parameters such as fuel rod length, upper free volume and fill-in pressure. Sensitivity studies have been performed on these parameters for the three rods and a final decision on the ones to be employed in the test has been made based on the sensitivities' outcomes.

\subsubsection{Irradiated fuel rod properties}

The fuel rod segment used in the experiment was irradiated in a commercial PWR plant. The table below is a summary of the main segment datasheet.

\begin{tabular}{|l|c|}
\hline \multicolumn{1}{|c|}{ Parameter } & Value \\
\hline Initial Density $(\%$ of $\mathrm{TD})$ & 96.1 \\
\hline Initial Enrichment $\left(\mathrm{w} / \mathrm{O}_{235}\right)$ & 3.8 \\
\hline Grain Size $(\mu \mathrm{m})$ & 8.5 \\
\hline Pellet Length $(\mathrm{mm})$ & 11.0 \\
\hline Initial Fuel - o.d. $(\mathrm{mm})$ & 9.12 \\
\hline Clad Material & $\mathrm{Zr}-4$ \\
\hline Initial Clad - o.d. $(\mathrm{mm})$ & 10.75 \\
\hline Initial Clad - i.d. $(\mathrm{mm})$ & 9.29 \\
\hline Initial Diameter gap $(\mu \mathrm{m})$ & 170.0 \\
\hline Average Burnup $\left(\mathrm{MWd} / \mathrm{kgUO}_{2}\right)$ & 52.0 \\
\hline
\end{tabular}

Table 2.2: Properties of the irradiated fuel segment employed in the test

The data of Table 2.2 were used to build the TRANSURANUS input for the irradiation calculation. TRANSURANUS can model the extremely complex material behaviors taking place during irradiation through a theoretical prediction of the physical, mechanical and chemical phenomena occurring. All key phenomena taking place in a fuel rod are dominated by the local temperature, and consequently the first task in modeling the fuel rod behavior is the calculation of temperature. However, the temperature is governed by the gap width which itself is controlled by deformations of the fuel and cladding. Obviously, the thermal and the mechanical analysis are interdependent and need to be determined 
consistently. These analyses have to take into account thermal expansion, deformations due to creep and plasticity, cracking and crack healing of the fuel, fuel densification and swelling etc., always taking into consideration that the irradiation causes complex changes in all these phenomena.

Determining the properties of the fuel is relevant to have a prediction of the properties of the fuel needed in the second step to discuss the outcomes of the separate effects test.

Figure 2.31 shows the power history of the rod segment expressed as Linear Heat Rate (LHR) for each of the four cycles as function of the rod average burnup. TRANSURANUS needs to have the linear heat generation rate as function of time and therefore the data of Figure 2.31 need to be translated in a new set of data LHR vs time.

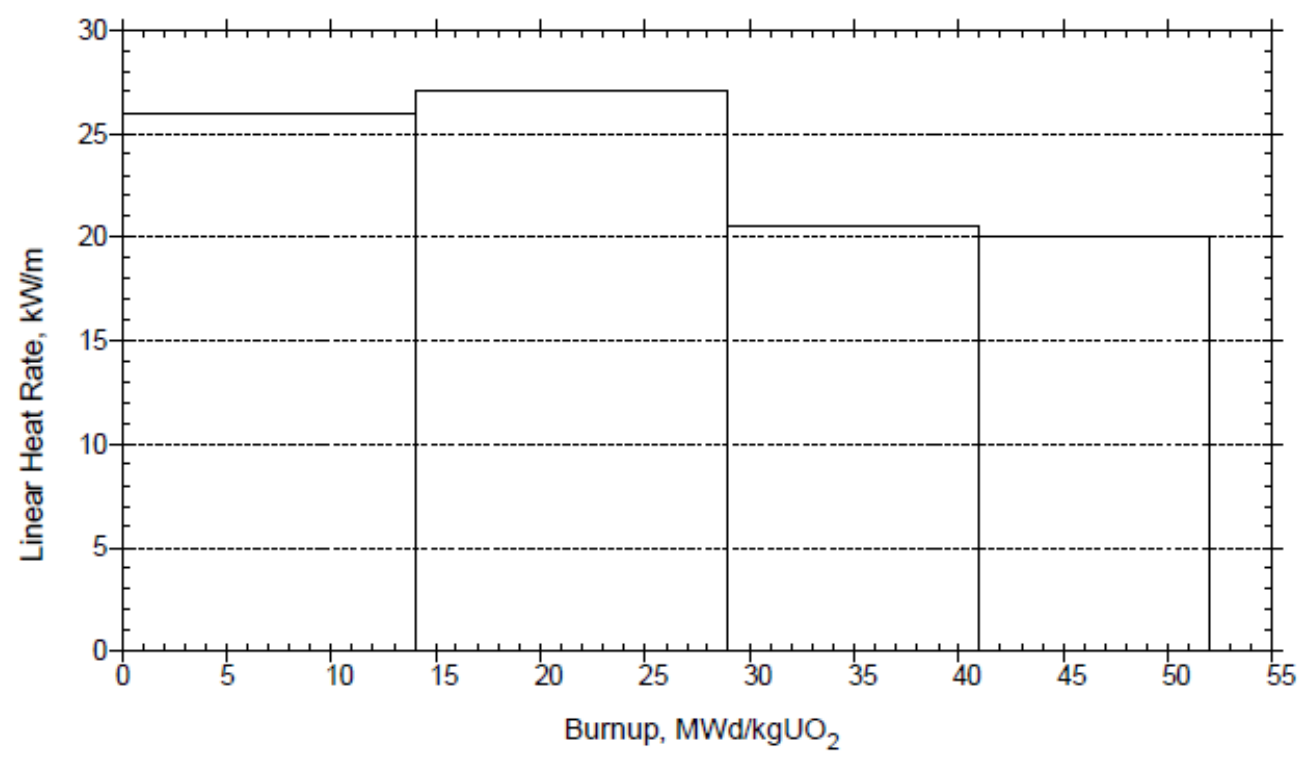

Figure 2.31: Power History

The burnup $\left[B U\left(\frac{W d a y}{k g_{U O_{2}}}\right)\right]$ can be expressed as function of time $[t(d a y)]$ and the specific power $\left[P S\left(\frac{W}{k g_{U O_{2}}}\right)\right]$

$$
\begin{gathered}
B U=P S \cdot t \quad(\text { Formula 2.3) } \\
P S=\frac{q^{\prime \prime \prime}}{\rho_{U O_{2}}} \cdot \frac{P M_{U O_{2}}}{P M_{U}} ; \quad \text { (Formula 2.4) }
\end{gathered}
$$


where:

$q^{\prime \prime \prime}\left[\frac{W}{m^{3}}\right]:$ Volumetric power;

$\rho_{U O_{2}}\left[\frac{k g_{U O_{2}}}{m^{3}}\right]: U O_{2}$ Density

$P M_{U O_{2}}[u($ atomic mass unit $)]: U O_{2}$ Molecular mass

$P M_{U}[u($ atomic mass unit $)]: U$ Molecular mass

Therefore for each cycle it is possible to determine the number of days to achieve the cycle burnup as follows:

$$
\Delta t_{i}=\frac{\Delta B U_{i}}{P S_{i}} \quad(\text { Formula 2.5) }
$$

Where the $\Delta()_{i}$ refers to the interval of time and burnup accumulated for each cycle $(i=1,2,3,4)$ and $P S_{i}$ is the specific power for each cycle.

The values of $\triangle B U_{i}$ can be obtained directly from Figure 2.31. The value of $P S_{i}$ can be calculated from the Formula 2.4:

$$
q^{\prime \prime \prime}=\frac{q^{\prime}}{\pi \cdot R_{f o}^{2}} \quad(\text { Formula 2.6) }
$$

$q^{\prime}\left[\frac{W}{m}\right]:$ LHR and it can be read for each cycle in Figure 2.31

$R_{f o}[m]:$ Radius of the fuel pellet (From Table 2.2)

$\rho_{U O_{2}}\left[\frac{\mathrm{kg}}{\mathrm{m}^{3}}\right]: U \mathrm{O}_{2}$ Density (from Table 2.2 expressed as \% of the uranium dioxide theoretical density $\left.10.97 \frac{\mathrm{g}}{\mathrm{cm}^{3}}\right)$

$$
\begin{gathered}
P M_{U O_{2}}=x \cdot P M_{U_{235}}+(1-x) \cdot P M_{U_{238}}+2 \cdot P M_{O_{2}} \quad \text { (Formula 2.7) } \\
P M_{U}=x \cdot P M_{U_{235}}+(1-x) \cdot P M_{U_{238}} \quad(\text { Formula 2.8) }
\end{gathered}
$$


In the previous two equations, $x$ represents the fuel enrichment in $U_{235}$ and the other parameters are the molecular masses of $U_{238}, U_{235}$ and $O_{2}$. This methodology is considered sufficiently accurate for the level of detail required by the investigation and in particular considering the level of accuracy of the fuel power history from Figure 2.31. Table 2.3 shows the data required by TRANSURANUS.

\begin{tabular}{|c|c|c|c|c|}
\hline cycle & $\mathbf{q}^{\prime}(\mathbf{W} / \mathbf{m})$ & $\mathbf{q}^{\prime \prime \prime}(\mathbf{W} / \mathbf{m} 3)$ & Ps (W/kg) & day \\
\hline $\mathbf{1}$ & 26000 & 398009822 & 42832.68 & 327 \\
\hline $\mathbf{2}$ & 27000 & 413317892 & 44480.09 & 337 \\
\hline $\mathbf{3}$ & 20500 & 313815437 & 33771.92 & 355 \\
\hline $\mathbf{4}$ & 20000 & 306161402 & 32948.22 & 334 \\
\hline
\end{tabular}

Table 2.3: Fuel rod power history data

The power history of the fuel segments was as input in TRANSURANUS (Figure 2.32). The fuel went through 4 cycles with the LHGR always smaller than $30 \mathrm{~kW} / \mathrm{m}$; therefore the fuel has not experienced any excessive LHGR during irradiation. It should be underlined that PWR fuel can experience during operation a maximum local power of $45-50 \mathrm{~kW} / \mathrm{m}$.

The LHGR has influence on the maximum centerline temperature and consequently on the temperature distribution. Figure 2.33 shows the fuel radial temperature distribution for the first and last cycle. Due to the average LHGR at the first cycles, the maximum centerline temperature is always smaller than 1100 ${ }^{\circ} \mathrm{C}$. The temperature distribution in the last cycle has been shown because Yhen [30] has been investigating the influence of the last cycle LHGR on fuel fragmentation. The fuel rods that have been used in the Halden and Studsvik tests have been characterized often by not standard power history with bigger than average LHGR in the last cycle of irradiation.

A relevant parameter to take in consideration is the dimension of the uranium dioxide grains after the manufacturing of the fuel and study if a grain growth process has occurred during irradiation. The grain growth model used in TRANSURANUS is based on the model of Ainscough [31]. Information about the model can be found also in [21]. Ainscough conducted a thorough investigation of equiaxial grain growth in uranium using samples with initial theoretical densities between 94 and 99\%, temperatures between 1273 and $1773 \mathrm{~K}$, and times up to 24 weeks. The densities and the O/M ratios of the samples remained constant during testing and showed little grain growth at temperatures below $1500 \mathrm{~K}$. Above $1500 \mathrm{~K}$, the grain growth rate increased rapidly with increasing temperatures. Since the maximum fuel temperature was limited during irradiation, no grain growth phenomena are predicted to occur as shown in Figure 2.34 .

As discussed in the previous chapter with the increase of the HBS thickness the fragmentation particles tend to decrease their sizes. The radial burnup distribution at the end of the irradiation is shown in Figure 2.35. Due to the radial variation of the neutron flux and energy spectrum inside $\mathrm{UO}_{2}$ fuel, the fission density and the fissile isotope production rates vary radially in the pellet [24]. During irradiation, fissile elements are generated by neutron absorption of fertile elements. The radial burnup distribution 
predicted by TRANSURANUS captures this behavior with the fuel burnup at the edge of the fuel of about $90 \mathrm{MWd} / \mathrm{kgHM}$ in comparison to the center of the fuel at about $45 \mathrm{MWd} / \mathrm{kgHM}$.

In terms of the development of the HBS, observing Figure 2.35, less than $0.1 \mathrm{~mm}$ was transformed to fully developed HBS and less than $0.4 \mathrm{~mm}$ was transformed to transition HBS. Considering the uncertainties of the calculation and of the power history of the fuel rod (especially only the average LHGR for each cycle is available), it can be concluded that the effect of the HBS is very limited to only a small fraction of fuel on the pellet edge.

The last investigation's subject covered by the study of the fuel after irradiation in the commercial reactor is the fission gas generation and release. During irradiation isotopes such as Xenon and Krypton are directly created inside the grains by fission and they also originate from decay processes. Generated in the grain, they tend to leave them by diffusion because of the very low solubility in $\mathrm{UO}_{2}$ or precipitate into intra- and inter-granular bubbles. Details of all these mechanisms are provided in $[6,25]$. TRANSURANUS allows determining the fraction of fission gases in the grain, in the grain boundaries, in the HBS and the fission gas release during irradiation. Figure 2.36 shows after irradiation the radial distribution of the parameters described above.

Most of the fission gas is retained in the fuel grains and partly in the grain boundaries. The amount of fission gas created increases with the fuel burnup and at the edge of the fuel there is a spike with the relocation of fission gas partly to the pores of the HBS and partly released in the fuel cladding gap. The total integral FGR has been estimated by TRANSURANUS to be approximately $0.35 \%$. The quantities calculated in terms of fission gas generation and release were used to make consideration on the expected fission gas release during the LOCA transient simulated in the oven. 


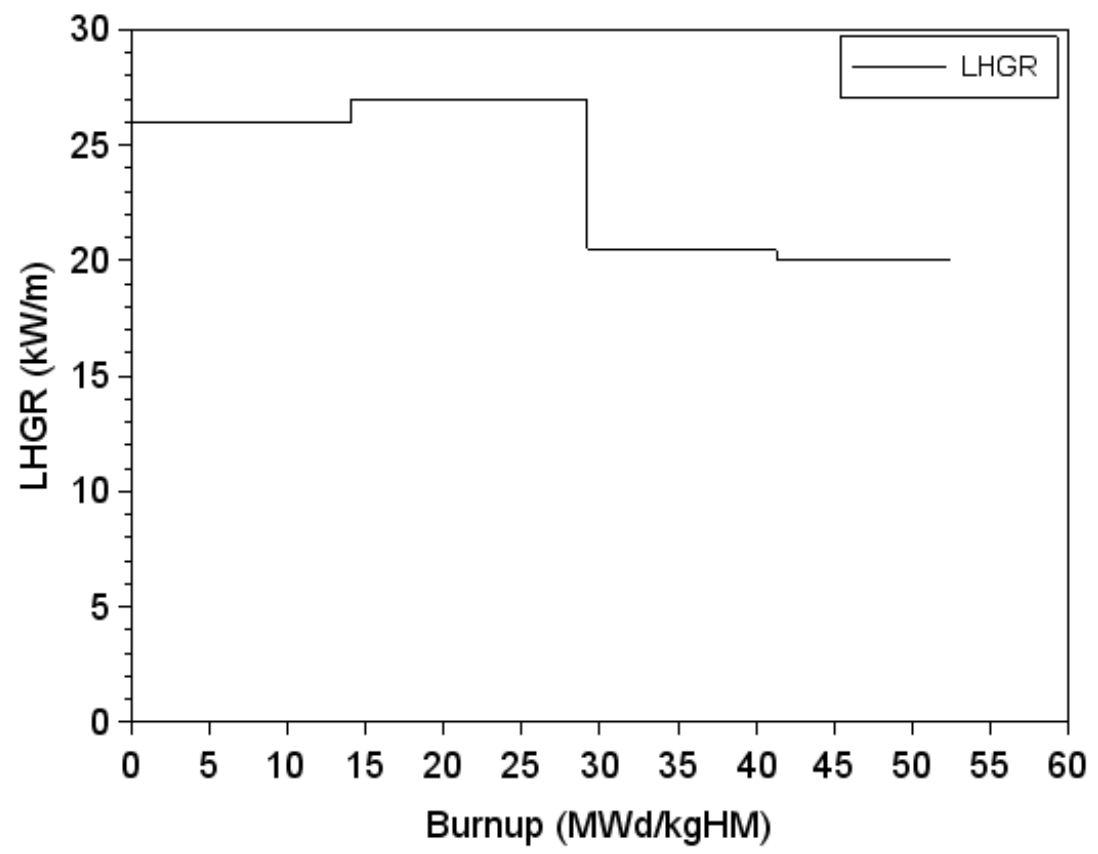

Figure 2.32: TRANSURANUS power history calculation

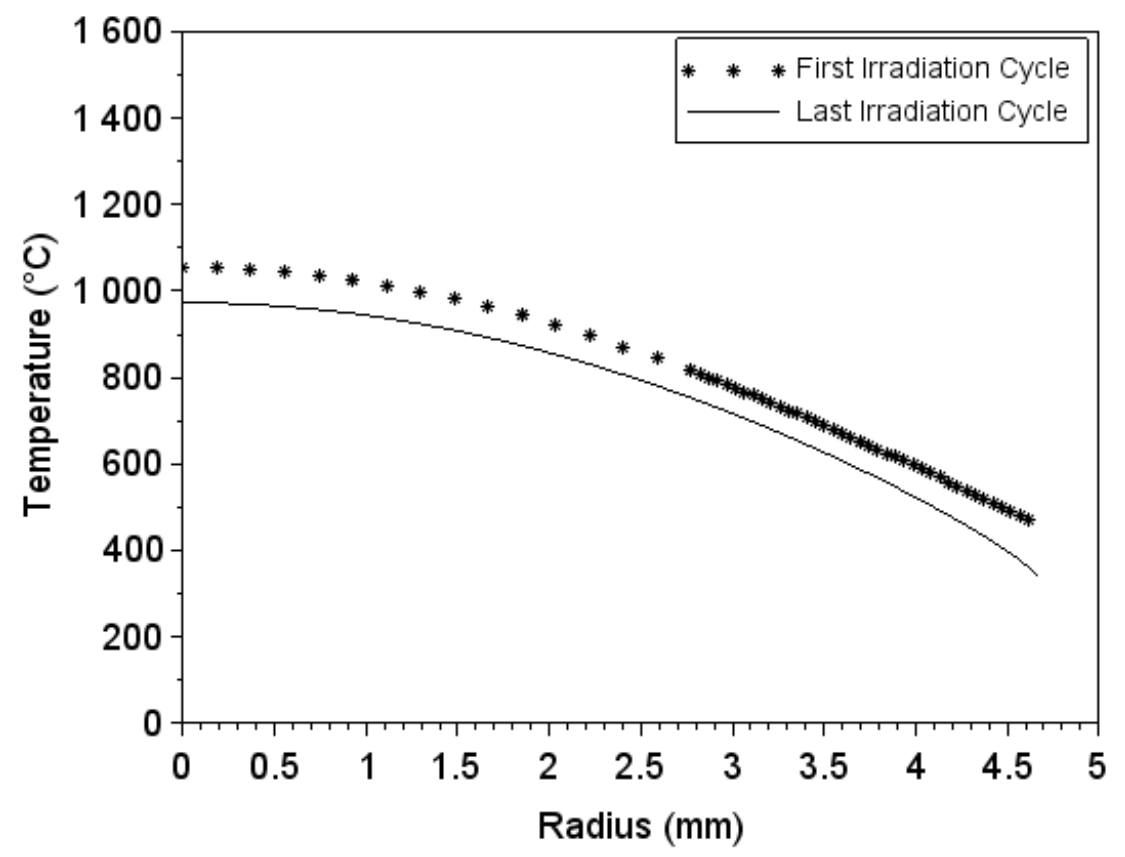

Figure 2.33: TRANSURANUS fuel temperature radial distribution calculation 


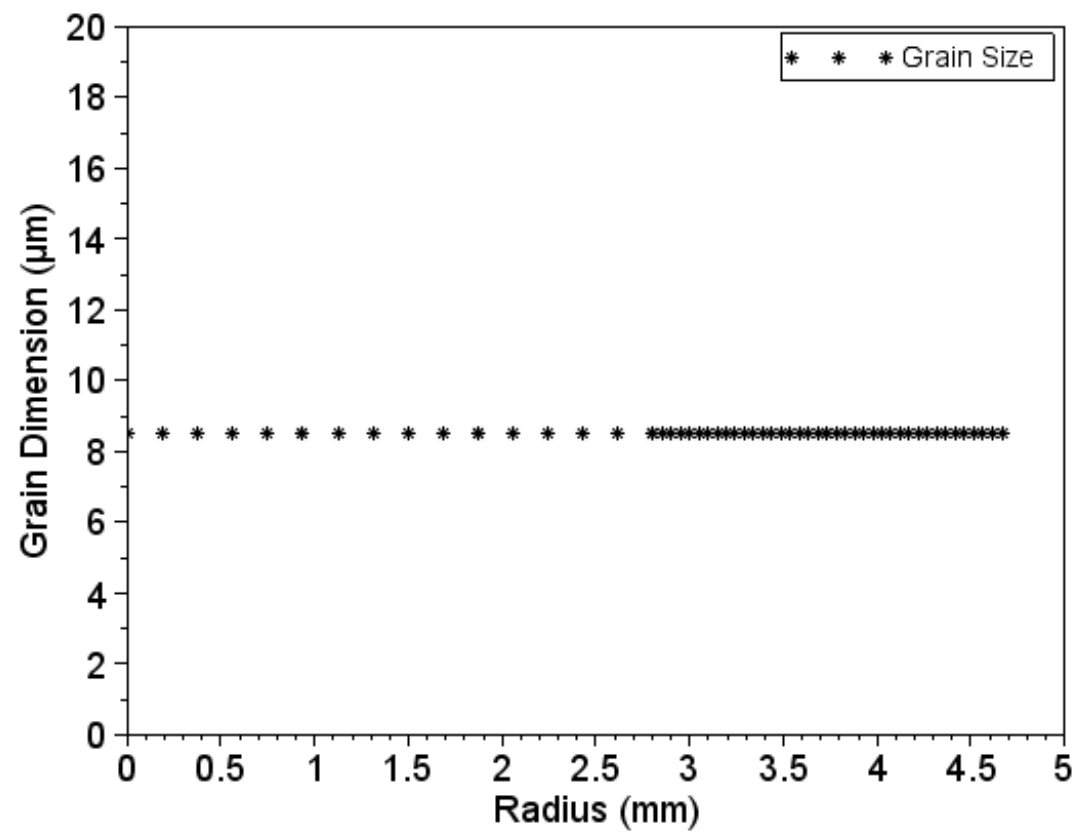

Figure 2.34: TRANSURANUS grain size after irradiation calculation

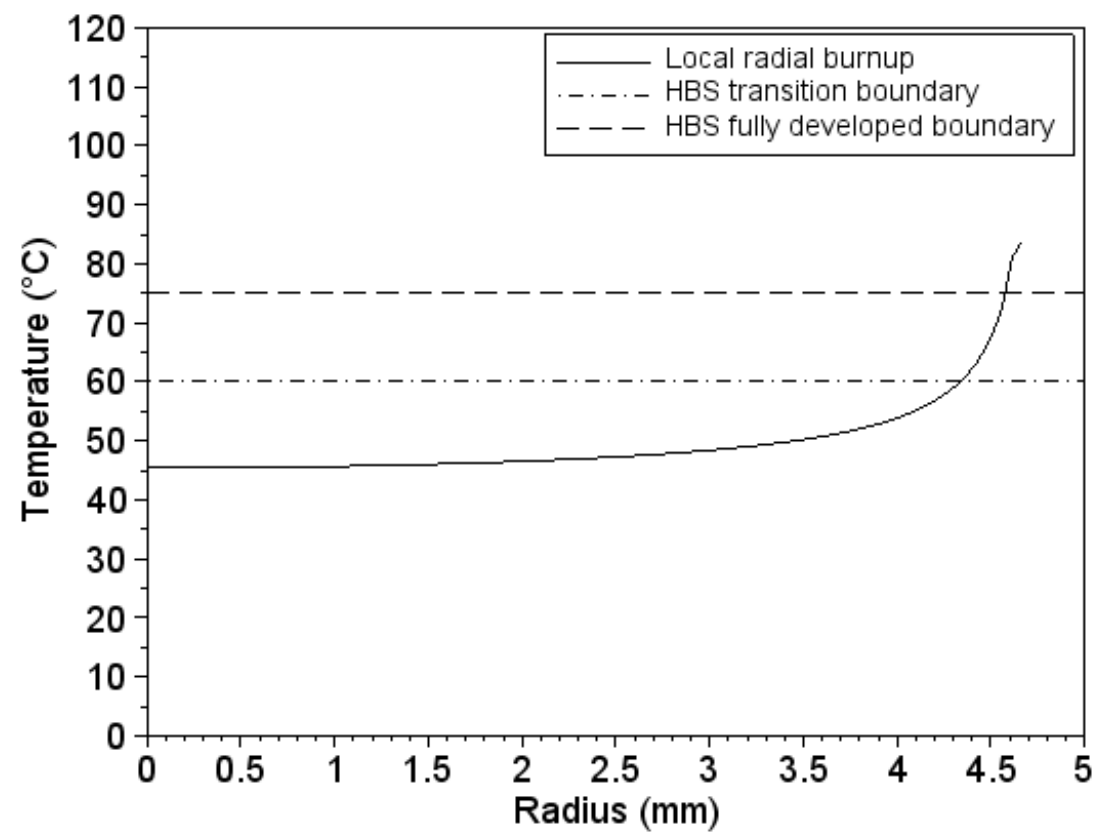

Figure 2.35: TRANSURANUS radial burnup and HBS boundaries calculation 


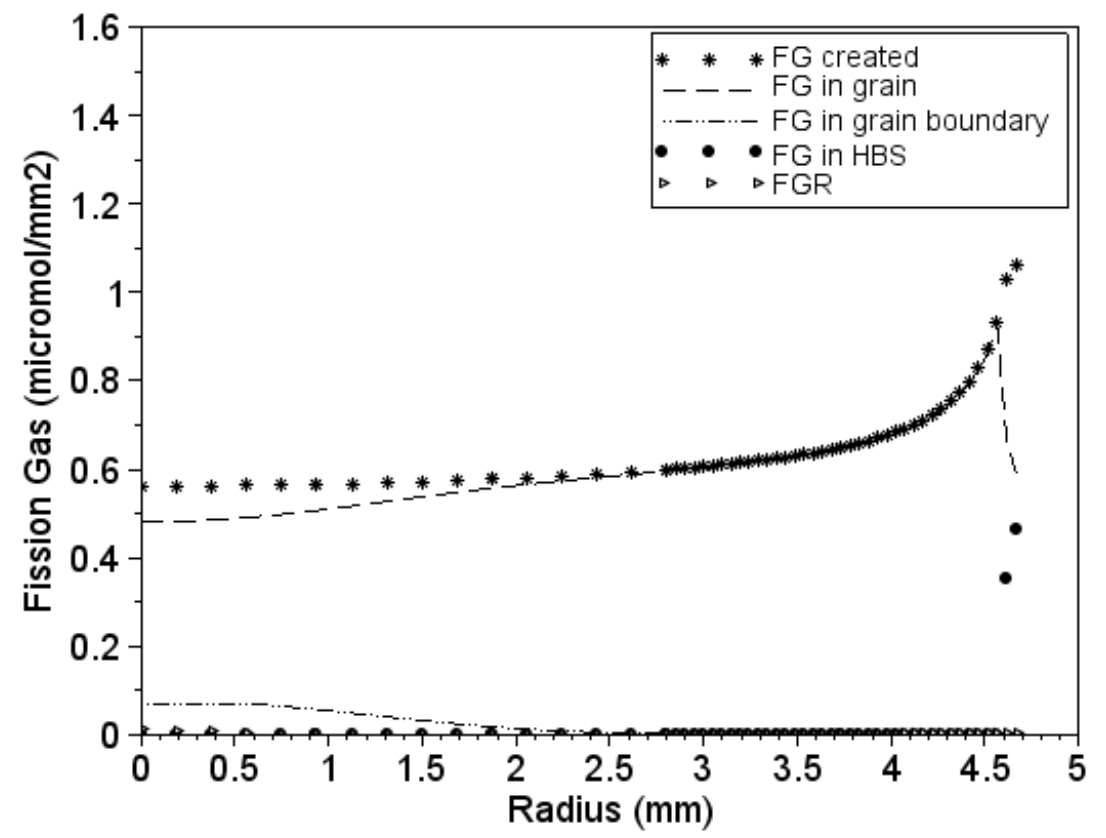

Figure 2.36: TRANSURANUS fission gases distribution after fuel irradiation calculation

\subsubsection{Selection of fuel rod design parameters}

The experiment modeling procedure developed in 2.2.3 with the validation of the codes used in 2.2.4 was used to determine the fuel rod design parameters to obtain the effects specified in 2.2.1. The boundary conditions to be obtained during the experiment are the following:

Rod 1. Fuel rod ballooning without cladding failure.

Rod 2. Fuel rod ballooning and burst (cladding failure).

Rod 3. No fuel rod ballooning (no cladding failure).

Under the simulated Loss Of Coolant Accident (LOCA) conditions, the cladding of the fuel rod is overheated and sustains extreme mechanical loading which leads to large plastic deformations and if some conditions are met, to the burst of the cladding tube.

TRANSURANUS calculates the large plastic deformation as non-elastic strain and the ballooning is also calculated incrementally in time [6]. The Zircaloy specific correlation is applied in a Norton-type form to describe the effective strain rate as a function of the effective stress and temperature. Furthermore, TRANSURANUS allows the simulation of the crystallographic phase transition at high temperature $\left(>600^{\circ} \mathrm{C}\right)$. Normally, extensive oxidation is observed in presence of a saturated steam environment and it influences the strength and the deformation of the cladding to a large extent. However, since the capsule environment is Helium, the oxidation model of the cladding during the heating up was 
deactivated and the only oxide on the cladding was the one accumulated during irradiation (corrosion of the cladding). The effect of the crystallographic phase transition on the deformation rate is simulated through the superposition of the effective strain rates defined separately for the zirconium-based alloys having HCP (Hexagonal Closed Pack) and BCC (Body Centered Cubic) crystal structures. The strain rates are weighted by the corresponding volumetric fractions of the two different phases. A description of the burst criteria mechanisms was given on 2.2.4.

In terms of fuel rod design parameters, the upper free volume and inner rod pressure play an important role to determine the extent of cladding deformation and occurrence of burst. Contrary to a real LOCA condition, the top part of the volume and therefore the upper free structure and volume are subject to the heating up even though it is slightly attenuated given the presence of the thermal insulation.

- Upper free volume: Given a fixed inner rod fill in pressure, the size of the upper free volume determines the amount of gas moles. The larger the size, the larger the amount of gas moles. The larger the size, the slower the decrease of pressure given by the occurrence of the ballooning with correspondent increase of the rod inner volume.

- Fill in gas pressure: The bigger the fill in gas pressure, the bigger the maximum pressure during the transient because of the upper free volume heating up. This value influences the beginning of the ballooning and in combination with the parameters above, the occurrence of burst.

Due to the physical explanation above, the following considerations were made concerning the three rods:

Rod 1. A sufficient level of inner rod pressure to ensure the beginning of the ballooning, but to ensure the avoidance of burst, a small upper free volume so that with the start of the ballooning the inner rod pressure quickly reduces.

Rod 2. A significant level of inner rod pressure to ensure the development of the ballooning and a large upper free volume so that the inner rod pressure does not quickly decrease and the ballooning is longer sustained and the burst criteria are met.

Rod 3. A level of pressure inside the rod comparable or smaller in respect of the capsule pressure (atmospheric) so that the difference of pressure between the inner and outer side of the rod is negligible.

In particular for Rod 1 and Rod 2 sensitivity studies have been performed to predict the behavior during the experiment. Rod 3 was considered easier to predict and no sensitivity analysis have been performed. The starting point fuel rod parameter design conditions are shown in Table 2.4.

\begin{tabular}{|l|c|c|c|}
\hline Parameter & Rod 1 & Rod 2 & Rod 3 \\
\hline Active Fuel Length (mm) & 11 & 14 & 11 \\
\hline Rod Fill in Pressure (bar) & 10 & 25 & 1 \\
\hline Upper free volume (cc) & 1 & 5 & 5 \\
\hline
\end{tabular}

Table 2.4: Fuel rod design parameters starting point 
The sensitivity on Rod 1 and 2 has been performed on the rod fill in pressure and on the axial temperature profile on the capsule and consequently on the cladding through a postulated peak scenario and less peak scenario.

Results of the sensitivity analysis can be found in Table 2.5 and Table 2.6 and representative plots of the analysis are show in Figures 2.37-2.40.

\begin{tabular}{|c|c|c|c|c|c|}
\hline Case ID & $\begin{array}{c}\text { Temperature } \\
\text { Profile }\end{array}$ & $\begin{array}{c}\text { Rod Inner } \\
\text { Pressure (bar) }\end{array}$ & Burst & $\begin{array}{c}\text { Maximum } \\
\text { Deformation }\end{array}$ & $\begin{array}{c}\text { Axial average } \\
\text { deformation }\end{array}$ \\
\hline Rod1_s0 & peak & 14 & yes & 22.25 & 13.75 \\
\hline Rod1_s1 & less_peak & 14 & no & 20 & 14.18 \\
\hline Rod1_s2 & less_peak & 11 & no & 15.75 & 13.08 \\
\hline Rod1_s3 & peak & 11 & no & 25 & 13.5 \\
\hline Rod1_s4 & peak & 10 & no & 19 & 13 \\
\hline Rod1_s5 & less_peak & 10 & no & 14.75 & 12.73 \\
\hline
\end{tabular}

Table 2.5: Rod 1 sensitivity analysis

\begin{tabular}{|c|c|c|c|c|c|}
\hline Case ID & $\begin{array}{c}\text { Temperature } \\
\text { Profile }\end{array}$ & $\begin{array}{c}\text { Rod Inner } \\
\text { Pressure (bar) }\end{array}$ & Burst time & $\begin{array}{c}\text { Maximum } \\
\text { Deformation }\end{array}$ & $\begin{array}{c}\text { Axial average } \\
\text { deformation }\end{array}$ \\
\hline Rod2_s0 & peak & 25 & 715 & 19.5 & 12.85 \\
\hline Rod2_s1 & less_peak & 25 & 740 & 21 & 13.4 \\
\hline Rod2_s2 & peak & 28 & 674 & 19.5 & 12.85 \\
\hline Rod2_s3 & less_peak & 28 & 695 & 21 & 13.5 \\
\hline Rod2_s4 & Peak & 20 & 812 & 11.3 & 11.8 \\
\hline Rod2_s5 & less_peak & 20 & 837 & 11.5 & 12.05 \\
\hline
\end{tabular}

Table 2.6: Rod 2 sensitivity analysis 

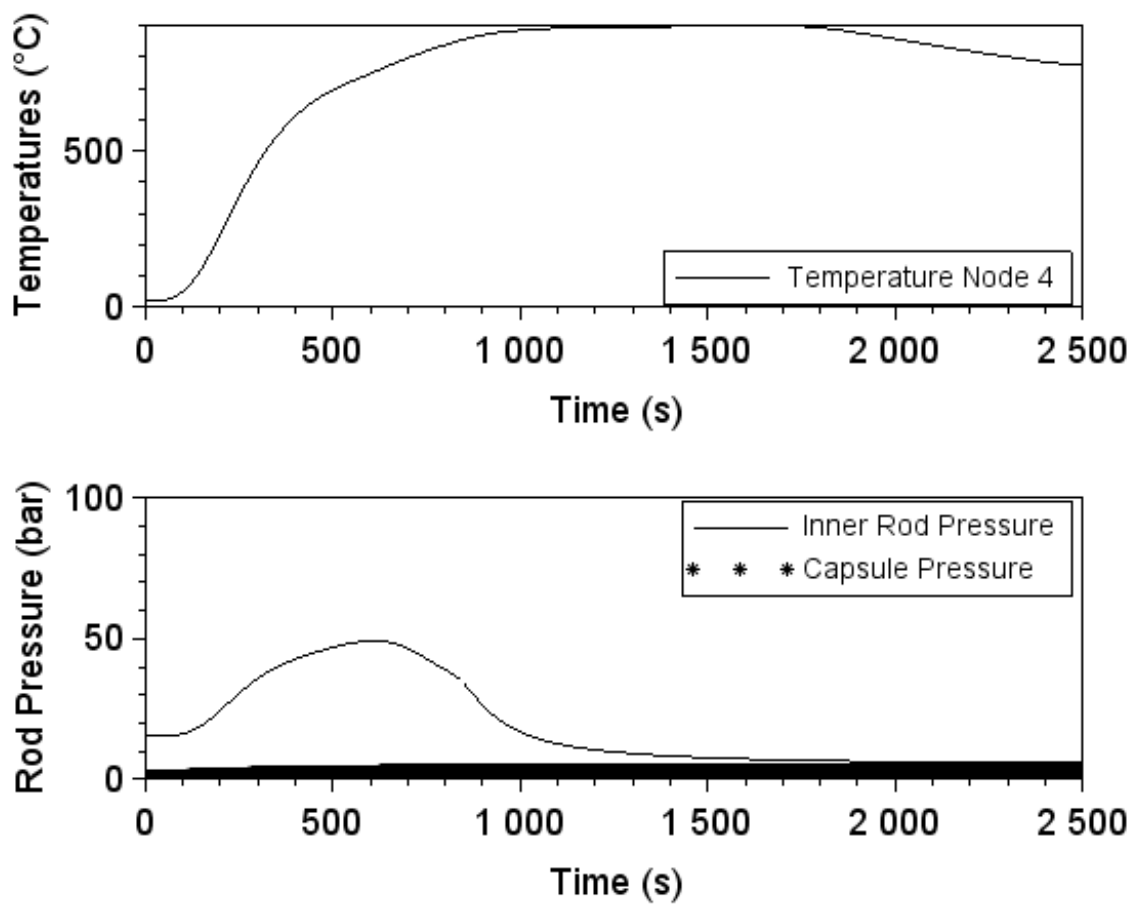

Figure 2.37: Rod1_s1 cladding temperature and inner rod pressure calculations Top Figure: cladding temperature; Bottom Figure: inner rod pressure transient
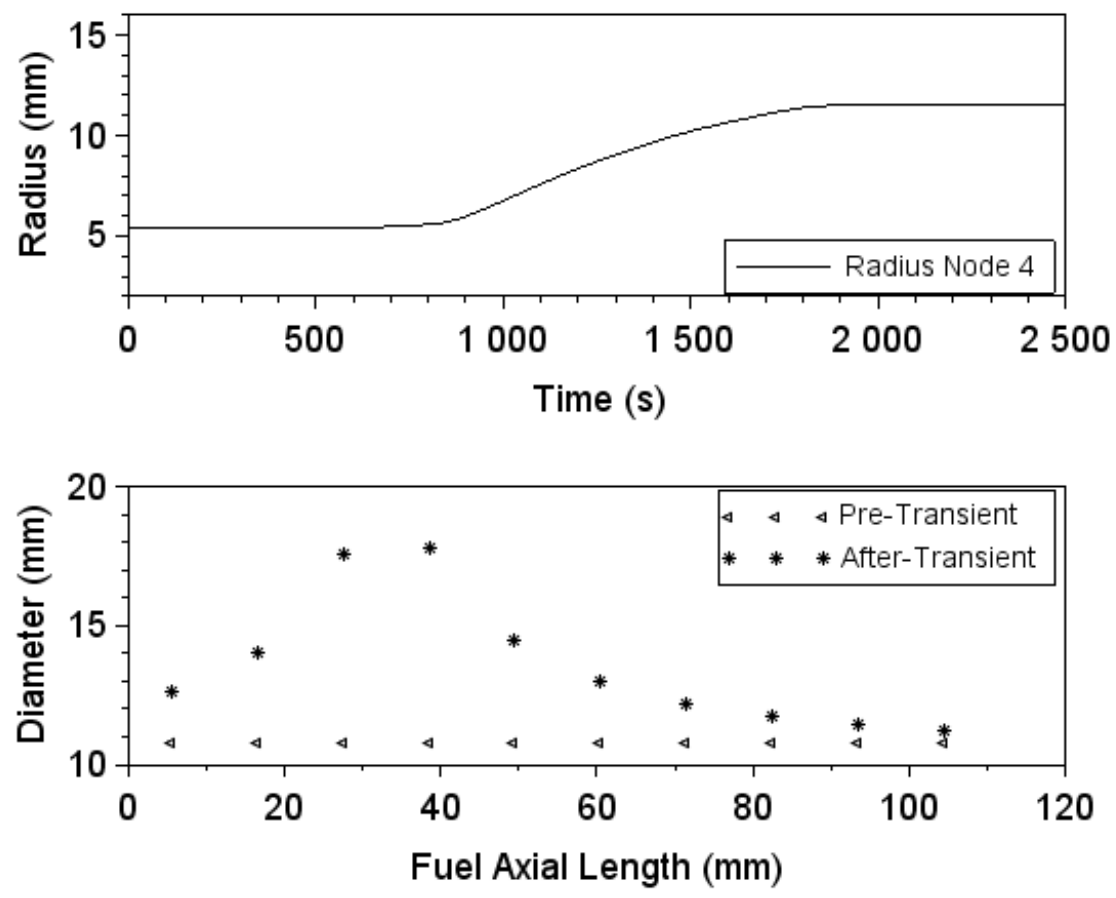

Figure 2.38: Rod1_s1 cladding deformation calculation Top Figure: cladding deformation vs. time; Bottom Figure: axial cladding deformation 

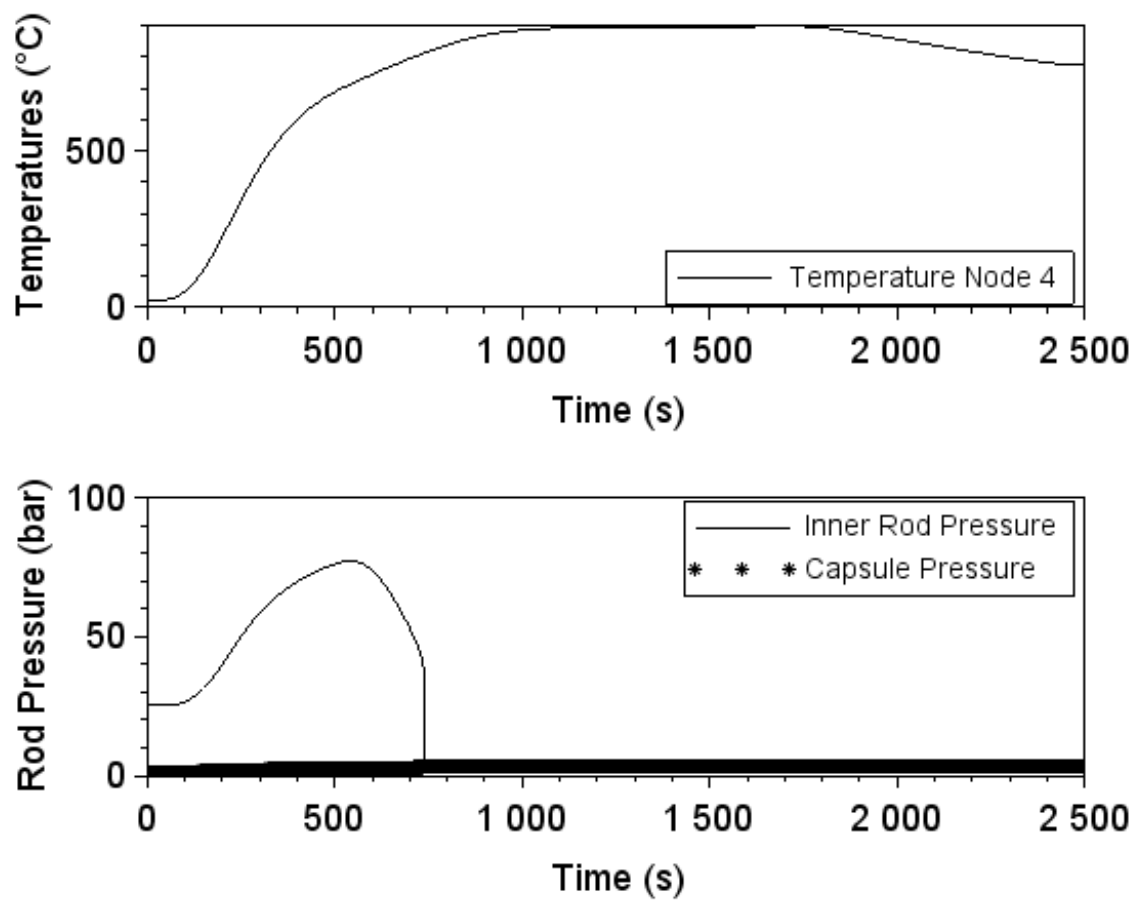

Figure 2.39: Rod2_s1 cladding temperature and inner rod pressure calculations Top Figure: cladding temperature; Bottom Figure: inner rod pressure transient
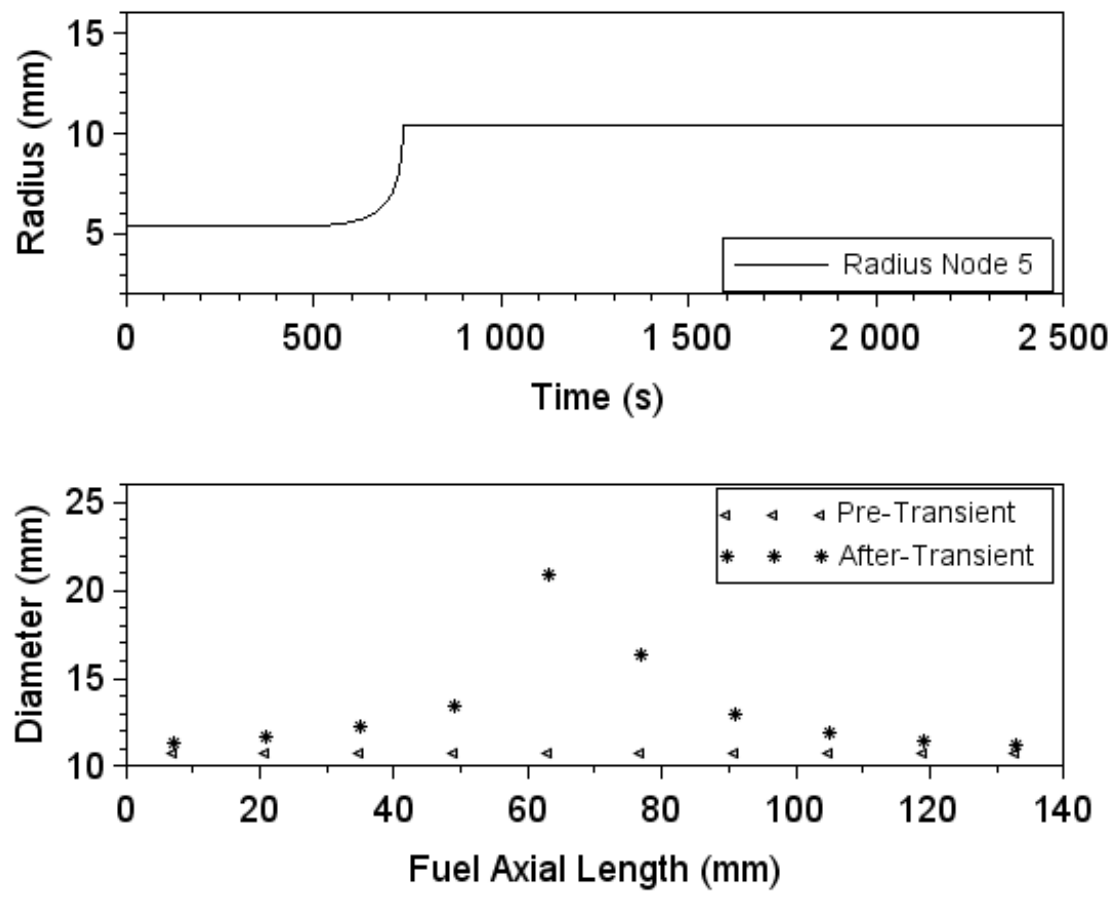

Figure 2.40: Rod2_s1 cladding deformation calculation Top Figure: cladding deformation vs. time; Bottom Figure: axial cladding deformation 
The sensitivity analysis can be summarized for the three rods as follows and the final rod design parameters are shown in Table 2.7.

- $\quad$ Rod 1: The sensitivity analysis shows a case (Rod1_s1) where the fuel rod experienced burst. This case was calculated with 14 bar rod internal pressure and a postulated peaked temperature profile. Additionally, all cases except the ones at 10 bar rod internal pressure show a maximum cladding deformation greater than $20 \mathrm{~mm}$. The contact between the cladding and the capsule must be avoided. Moreover, the calculations do not consider any potential fission gas released during the transient with an additional increase of the inner rod pressure that may correspond to further cladding deformation. Based on these considerations, it was decided to set the rod internal pressure at the smallest value of Table 2.5 corresponding to $10 \mathrm{bar}$.

- Rod 2: All the sensitivities of Table 2.6 show that the fuel rod burst and the results in terms of cladding deformation are comparable. Consequently, the inner rod pressure value of 25 bar was confirmed as selected value.

- $\quad$ Rod 3: No sensitivities have been performed. However, to ensure that the rod inner pressure is always smaller than the capsule pressure, the fill in pressure value was set to 0.7 bar with the capsule pressure set at 1.0 bar.

\begin{tabular}{|l|c|c|c|}
\hline Parameter & Rod 1 & Rod 2 & Rod 3 \\
\hline Active Fuel Length (mm) & 11 & 14 & 11 \\
\hline Rod Fill in Pressure (bar) & 10 & 25 & 0.7 \\
\hline Upper free volume (cc) & 1 & 5 & 5 \\
\hline
\end{tabular}

Table 2.7: Selected final fuel rod design parameters 


\subsection{Fission gas release during LOCA}

A study of the effect of the transient fission gas release during LOCA was performed with the following objectives:

- Make an assessment based on the current available data on the amount of fission gas release during a LOCA.

- Study the effect the transient fission gas release has during the separate effects test. Additional fission gas moles in the plenum increase the rod inner pressure, causing a stronger ballooning. The phenomenon required particular attention for the design of Rod 1 to avoid that this increase of pressure would have brought to a rod burst. The outcomes of this investigation were used to confirm the validity of the rod design parameters of Table 2.7, because the TRANSURANUS calculation did not include a refined model to evaluate the fission gas release during the transient that includes fuel fragmentation and pulverization of the HBS.

- The fission gas release during the transient was experimentally investigated and a solid background was required to implement the correct design features and procedures to have reliable measurements.

The analysis is based on the study of the behavior of the transient fission gas release during the LOCA test IFA-650.12 [28]. PSI performed a study to assess the fission gas release during IFA-650.12 and estimated an amount of fission gas release of $14 \%$ of the total fission gas retained in the fuel matrix. Based on their conclusions the source of release is mainly pores of the HBS. An ATHLET-TRANSURANUS calculation as shown in 2.2.4 was done for IFA-650.12 and an independent transient fission gas release assessment was performed.

The IFA-650 LOCA tests have been characterized by large upper free volumes. In addition, the upper free volume was the sum of volumes at different locations: one part is the upper free volume on the top of the rod at the experimental rig temperature, another part is on the top of the experimental rig in an unheated area and a third part is the channel outside the experimental rig that connects the rod upper free volume to the pressure transducer. The latter is at the temperature condition of the Halden Boiling Water Reactor. Considering the uncertainties in assessing the temperature of these three parts of the upper plenum, it has been very challenging to establish a relation between the transient fission gas release and the increase of pressure in the upper plenum. However, the IFA- 650.12 test was performed with a very small upper free volume $(2 \mathrm{cc})$ and this makes possible a transient fission gas release evaluation.

Figure 2.41 shows a comparison between the TRANSURANUS prediction of the rod internal pressure and the experimental data for IFA-650.12. It can be observed that the TRANSURANUS underestimated the maximum pressure reached during the experiment. Moreover the burst criterion is not met in TRANSURANUS, while in the last phase of the test (some seconds after the SCRAM), fuel rod burst occurred. 
Three points can be identified observing the evolution of the inner rod pressure and top cladding thermocouple during the LOCA test as shown in Figure 2.42.

- Point 1: Start of the inner rod pressure transient (corresponding to the experimental rig dry out)

- Point 2: Start of the ballooning

- Point 3: Burst criterion met

The gas law for the three points can be written as follow:

$$
\begin{array}{ll}
p_{1} \cdot V_{1}=n_{1} \cdot R \cdot T_{1} & \text { (Formula 2.9) } \\
p_{2} \cdot V_{2}=n_{2} \cdot R \cdot T_{2} & (\text { Formula 2.10) } \\
p_{3} \cdot V_{3}=n_{3} \cdot R \cdot T_{3} & (\text { Formula 2.11) }
\end{array}
$$

- $\quad V_{1} \approx V_{2}$ : The free volume is given by the actual volume of the upper free volume plus the space available for the instrumentation. The latter does not change unless the rod starts to balloon (point 2). The hypothesis is valid considering the effect of the upper free volume deformation during the transient as negligible.

- $n_{1}$ : it is the number of moles of the fill-in gas plus the value of fission gas release due to the irradiation time in the Halden Reactor after rod remanufacturing (considered negligible).

- $n_{2}=n_{1}+\Delta n_{1 \rightarrow 2}^{F G R}$ : The number of moles from point 1 to 2 increases due to the fission gas release transient from 1 to 2 .

- $\quad V_{3}=V_{1}+\Delta V_{\text {ballooning }}$ : The free volume in the rod increases due to the cladding deformation. An estimation of this volume can be made based on the cladding post experiment examination and looking at the axial cladding diameter distribution. [28] estimated $\Delta V_{\text {ballooning }}=8.4 \mathrm{cc}$.

- $n_{3}=n_{2}+\Delta n_{2 \rightarrow 3}^{F G R}$ the number of moles at the point 3 considers also the fission gas release transient between point 2 and 3 .

Considering that the values of pressure and temperature can be read by Figure 2.42, all information needed to obtain the missing variables $\left(\Delta n_{1 \rightarrow 2}^{F G R} ; \Delta n_{2 \rightarrow 3}^{F G R}\right)$ equalizing the gas law between point 1 and 2 and then between point 2 and 3 are available. It should be underlined that the cladding temperature from Figure 2.42 corresponds to the top thermocouples and the distance between it and the plenum is about $6 \mathrm{~cm}$. 


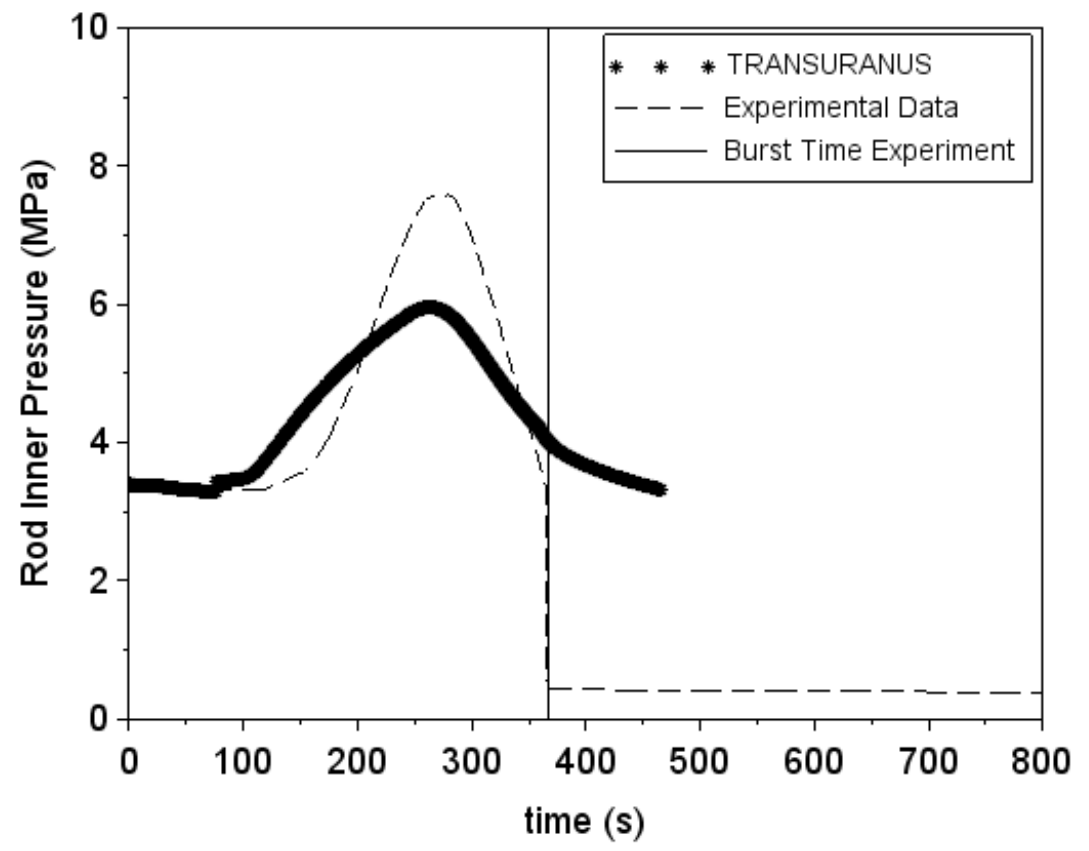

Figure 2.41: TRANSURANUS IFA-650-12 benchmark calculation
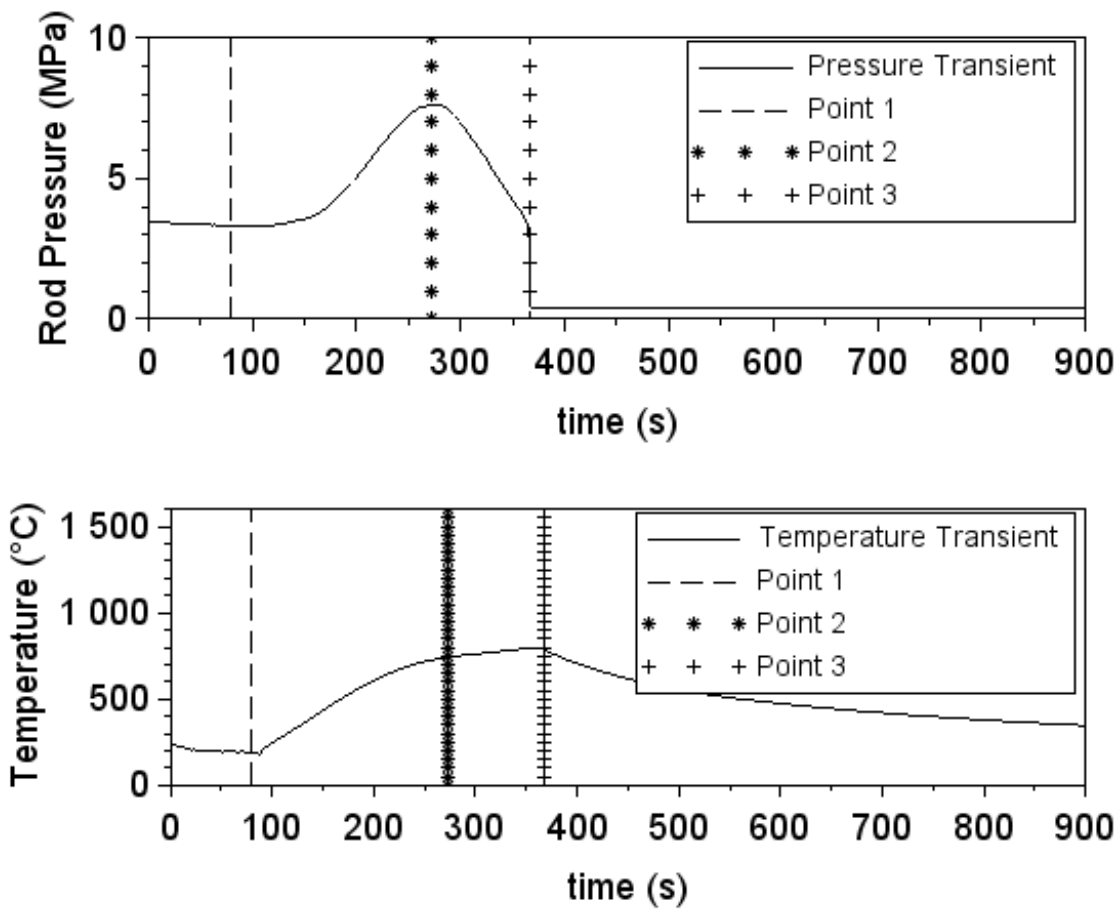

Figure 2.42: Evolution of rod pressure and cladding temperature IFA-650.12 
The evaluation starts with the calculation of the initial moles $n_{1}$ :

$T_{1}=200^{\circ} \mathrm{C}=473.15 \mathrm{~K}$

$p_{1}=3.25 \mathrm{MPa}=32.075 \mathrm{~atm} ;$

$V_{1}=2.3 c c=0.0023 \mathrm{~L}$

$R=0.08206 \frac{\mathrm{atm} \cdot \mathrm{L}}{\mathrm{mol} \cdot \mathrm{K}}$

$$
n_{1}=\frac{p_{1} \cdot V_{1}}{R \cdot T_{1}}=0.0019 \mathrm{~mol} \quad \text { (Formula 2.12) }
$$

Gas Law conservation (at constant volume) between point 1 and 2 and calculation of moles $n_{2}$ :

$p_{1}=7.60 \mathrm{MPa}=75.06 \mathrm{~atm}$

$T_{2}=720^{\circ} \mathrm{C}=993.15 \mathrm{~K}$;

$$
n_{2}=\frac{p_{2}}{p_{1}} \cdot \frac{T_{1}}{T_{2}} \cdot n_{1}=0.0021183 \mathrm{~mol} \quad \text { (Formula 2.13) }
$$

The Gas Law can also be applied between point 1 and 3 to obtain the moles $n_{3}$ :

$T_{3}=800^{\circ} \mathrm{C}=1073.15 \mathrm{~K}$;

$p_{3}=3.25 \mathrm{MPa}=32.075 \mathrm{~atm} ;$

$V_{3}=(2.3+8.4) c c=10.7 c c=0.0107 \mathrm{~L} ;$

$$
n_{3}=\frac{p_{3}}{p_{1}} \cdot \frac{V_{3}}{V_{1}} \cdot \frac{T_{1}}{T_{3}} \cdot n_{1}=0.0038972 \mathrm{~mol} \quad \text { (Formula 2.14) }
$$

Estimations the fission gas release transient impacts between point 1 and 2 and point 2 and 3 and their contribution in percentage to the entire transient fission gas can be made based on the following:

$$
\begin{aligned}
& \Delta n_{1 \rightarrow 2}^{F G R}=n_{2}-n_{1}=0.0002183 \mathrm{~mol} \text { (Formula 2.15) } \\
& \Delta n_{2 \rightarrow 3}^{F G R}=n_{3}-n_{2}=0.0017789 \mathrm{~mol} \text { (Formula 2.16) } \\
& \Delta n_{1 \rightarrow 3}^{F G R}=n_{3}-n_{1}=0.0019972 \mathrm{~mol} \quad \text { (Formula 2.17) } \\
& \frac{\Delta n_{1 \rightarrow 2}^{F G R}}{\Delta n_{1 \rightarrow 3}^{F G R}}=10.9 \% \text { (Formula 2.18) } \\
& \frac{\Delta n_{2 \rightarrow 3}^{F G R}}{\Delta n_{1 \rightarrow 3}^{F G R}}=89.1 \% \text { (Formula 2.19) }
\end{aligned}
$$


A correlation is required to calculate from the moles released during the transient the percentage of fission gas release with respect to the fission gas produced during fuel irradiation. From the irradiation calculation of IFA-650.12 with TRANSURANUS the total amount of fission gas generated and released during irradiation can be determined. The amount of fission gas release in terms of moles can be estimated as $0.0005866 \mathrm{~mol}$. This value corresponds to an integral value of fission gas release during irradiation of $4.20 \%$. Applying the proportions of below, the transient fission gas releases for the two phases of LOCA $\left(F G R_{1 \rightarrow 2}^{\%}\right.$ and $\left.F G R_{2 \rightarrow 3}^{\%}\right)$ and the total transient FGR $\left(F G R_{1 \rightarrow 3}^{\%}\right)$ can be calculated:

$$
\begin{aligned}
& n_{\text {irradiation }}^{F G R}: F G R_{\text {irradiation }}^{\%}=\Delta n_{1 \rightarrow 2}^{F G R}: F G R_{1 \rightarrow 2}^{\%} \quad(\text { Formula 2.20) } \\
& n_{\text {irradiation }}^{F G R}: F G R_{\text {irradiation }}^{\%}=\Delta n_{2 \rightarrow 3}^{F G R}: F G R_{2 \rightarrow 3}^{\%} \quad(\text { Formula 2.21) } \\
& n_{\text {irradiation }}^{F G R}: F G R_{\text {irradiation }}^{\%}=\Delta n_{1 \rightarrow 3}^{F G R}: F G R_{1 \rightarrow 3}^{\%} \quad(\text { Formula 2.22) }
\end{aligned}
$$

The resulted values are the following:

$F G R_{1 \rightarrow 2}^{\%}=1.56 \%$

$F G R_{2 \rightarrow 3}^{\%}=12.74 \%$

$F G R_{1 \rightarrow 3}^{\%}=14.30 \%$

The total fission gas release transient is estimated to be about $14 \%$ and this value is consistent with the analysis of [28].

An understanding of the origin of the fission gas release during the transient is required. The neutron radiography of the fuel after the test is shown in Figure 2.43 [29]. 


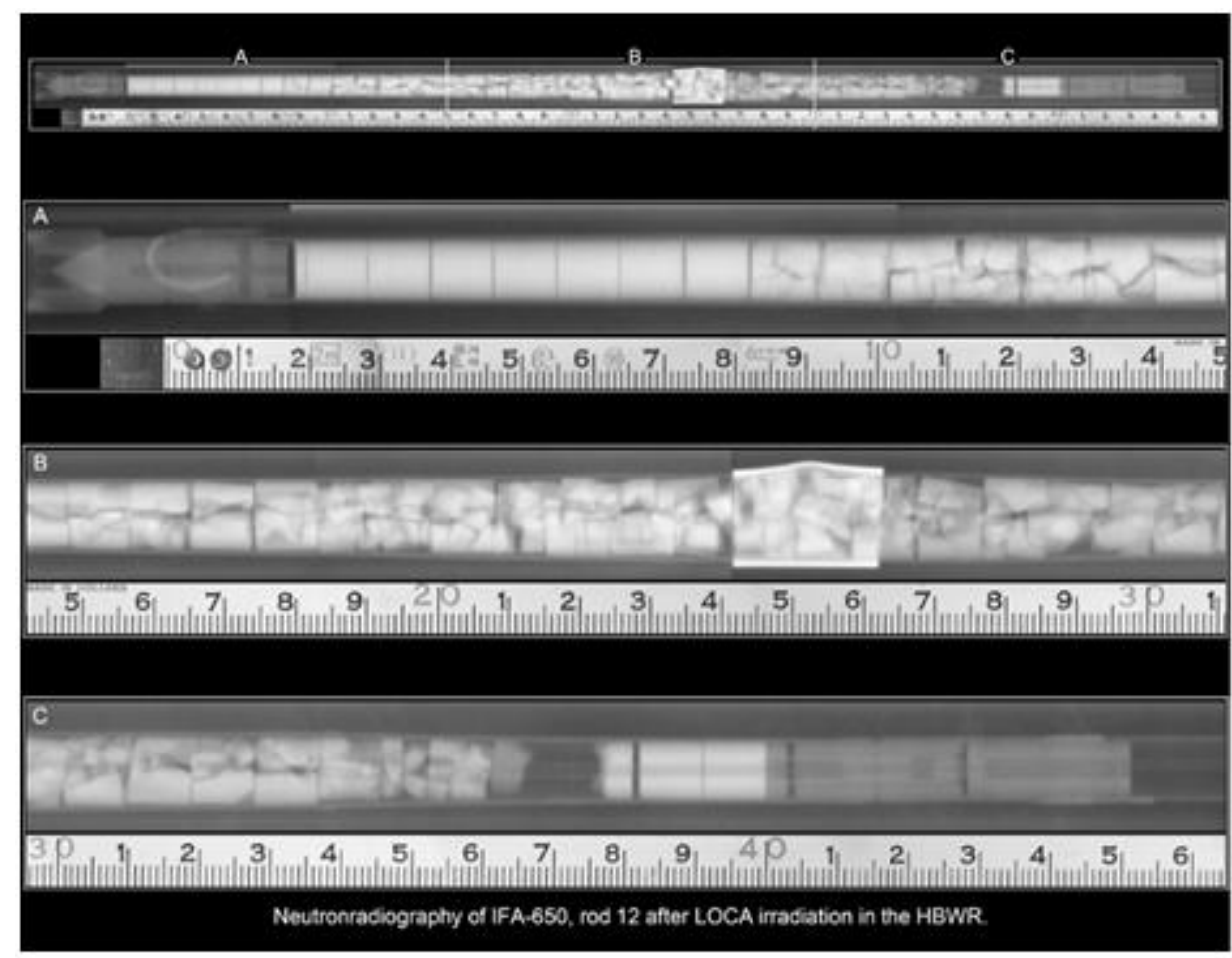

Figure 2.43: Neutron radiography IFA-650.12

Figure from Reference [29]

To quantify the amount of gas present in the HBS after irradiation, some considerations are needed. The fuel rod that has been used has a relatively high burnup $72 \mathrm{MWd} / \mathrm{kgU}$. Consequently, the burnup in the outer region of the fuel reaches very high values at the end of irradiation. TRANSURANUS applies for the fission gas release from the high burnup structure the so-called "saturation approach". As soon as the local burnup exceeds an empirical threshold (present standard value: bu2 $=85 \mathrm{MWd} / \mathrm{kgHM}$ ), the HBS is assumed to be saturated, i.e. all additionally arriving fission gas is immediately released to the free volume. Since this mechanism has not been fully understood and there are examples from the literature that show retention up to $100 \mathrm{MWd} / \mathrm{kgHM}$, a sensitivity analysis has been performed with different thresholds: $85 \mathrm{MWd} / \mathrm{kgHM}, 90 \mathrm{MWd} / \mathrm{kgHM}, 95 \mathrm{MWd} / \mathrm{kgHM}, 100 \mathrm{MWd} / \mathrm{kgHM}$ and the results are shown in Table 2.7.

\begin{tabular}{|c|c|c|c|c|c|}
\hline $\begin{array}{l}\text { Case } \\
\text { SaturationThreshold } \\
(\mathbf{M W d / k g H M )}\end{array}$ & $\begin{array}{l}\text { Total } \\
\text { Fission Gas } \\
\text { Production } \\
\left(\mathrm{cm}^{3}\right)\end{array}$ & $\begin{array}{l}\text { Integral } \\
\text { FGR (\%) }\end{array}$ & $\begin{array}{l}\text { Total } \\
\text { Fission Gas } \\
\text { Production } \\
\text { Node } 5 \\
\left(\frac{\mu m o l}{\mathrm{~mm}}\right)\end{array}$ & $\begin{array}{lr}\text { Total } \\
\text { Fission } \\
\begin{array}{lr}\text { in } & \text { HBS } \\
\text { Node } \\
\left(\frac{\mu m o l}{\mathrm{~mm}}\right)\end{array} \\
\end{array}$ & $\begin{array}{l}\text { Ratio } \\
\text { Fission Gas } \\
\text { in HBS / } \\
\text { Fission Gas } \\
\text { Production } \\
(\%)\end{array}$ \\
\hline 85000 (default) & 404.81 & 4.20 & 47.08981 & 4.30754 & 9.15 \\
\hline 90000 & 404.81 & 2.54 & 47.08994 & 5.14995 & 10.93 \\
\hline 95000 & 404.81 & 1.60 & 47.08994 & 5.65785 & 12.01 \\
\hline 100000 & 404.81 & 1.05 & 47.08994 & 5.98386 & 12.71 \\
\hline
\end{tabular}

Table 2.8: Sensitivity on TRANSURANUS grain boundaries saturation approach 
As it can be observed, the fission gas retained in the HBS increases when the saturation threshold increases. Based on the most recent investigation that shows full FG-retention in the HBS up to 90000 , $100000 \mathrm{MWd} / \mathrm{kgHM}$, the integral fission gas release during irradiation would result in values between 1.05 and $2.54 \%$.

It is important to focus the attention on the FGR retained in the HBS. Considering the evaluation sensitivities the fission gases in the HBS are between $9.15 \%$ and $12.71 \%$ of the total fission gas generation and above the LOCA transient fission gas release is estimated to be $14.30 \%$. Based on these results, an interpretation of the origin of the fission gas release during the transient can be given as follows: most of the fission gases of the HBS is released (between 9.15\% and $12.71 \%$ ) and in addition other fission gas is released from the fuel matrix mainly because of the cracks due to the fragmentation and the diffusion following the transient heating up (between $1.59 \%$ and $5.15 \%$ ). The results are summarized in Table 2.9.

\begin{tabular}{|l|c|c|c|}
\hline Case & Transient FGR & HBS Release & Release Matrix \\
\hline $\begin{array}{l}\text { Calculation with low fission gas retention in } \\
\text { HBS ("HBS saturation" 85000 MWd/kgHM) }\end{array}$ & $14.30 \%$ & $9.15 \%$ & $5.15 \%$ \\
\hline $\begin{array}{l}\text { Calculation with high fission gas retention in } \\
\text { HBS ("HBS saturation" } 100000 \mathrm{MWd} / \mathrm{kgHM} \text { ) }\end{array}$ & $14.30 \%$ & $12.71 \%$ & $1.59 \%$ \\
\hline
\end{tabular}

Table 2.9: Summary of results of transient FGR on IFA-650.12

Making similar considerations with a fuel rod with an average burnup of $52 \mathrm{MWd} / \mathrm{kgU}$ as the irradiated fuel rod to be used in the separate effect tests, the HBS fission gas saturation threshold is not relevant because only a very small thickness of the fuel is in a fully developed HBS (Figure 2.35). Results of this calculation are shown in Table 2.10.

\begin{tabular}{|c|c|c|c|c|c|}
\hline $\begin{array}{c}\text { Case SaturationThreshold } \\
(\mathbf{M W d} / \mathrm{kgHM})\end{array}$ & $\begin{array}{c}\text { Total Fission } \\
\text { Gas } \\
\text { Production } \\
\left(\mathbf{c m}^{\mathbf{3}}\right)\end{array}$ & $\begin{array}{c}\text { Integral } \\
\text { FGR }(\%)\end{array}$ & $\begin{array}{c}\text { Total Fission } \\
\text { Gas } \\
\text { Production } \\
\text { Node } \mathbf{5}\left(\frac{\boldsymbol{\mu m o l}}{\mathbf{m m}}\right)\end{array}$ & $\begin{array}{c}\text { Ratio Fission } \\
\text { Gas in HBS } \\
\text { Node } \mathbf{5}\left(\frac{\mu m o l}{\mathbf{m m}}\right)\end{array}$ & $\begin{array}{c}\text { Fas in HBS / } \\
\text { Fission Gas } \\
\text { Production } \\
(\%)\end{array}$ \\
\hline $\mathbf{8 5 0 0 0}$ & 100.33 & 0.35 & 44.45213 & 0.857197 & 1.93 \\
\hline
\end{tabular}

Table 2.10: TRANSURANUS fission gas results for Rod 2

Considering the smaller fuel rod average burnup (52 vs. $72 \mathrm{MWd} / \mathrm{kgU}$ ), it is easy to explain the differences between the two tables: smaller fission gas production, smaller fission gas release and smaller percentage of fission gas release in the developed HBS.

Assuming that the fission gas from the HBS is fully released, two cases can be considered in terms of fission gas release from the fuel matrix as for IFA-650.12. In fact, Table 2.9 shows that depending on the 
methodology used to estimate the retention of FGR in the HBS, the release from the matrix can be between $1.59 \%$ and $5.15 \%$.

Assuming that the release from the matrix is similar between IFA-650.12 and Rod 2, it is possible to estimate the release from Rod 2 as shown in Table 2.11. The table considers two different cases: 1) a small fission gas release from the matrix because of fragmentation (1.59\%); 2) a large fission gas release from the matrix because of fragmentation (5.15\%). Rod 2 was explicitly selected because IFA-650.12 experienced ballooning and burst.

\begin{tabular}{|c|c|c|c|}
\hline Case & $\begin{array}{c}\text { Release from the } \\
\text { matrix }\end{array}$ & Release from HBS & Total transient FGR \\
\hline $\begin{array}{c}\text { Low fission gas release } \\
\text { from the matrix }\end{array}$ & $1.59 \%$ & $1.93 \%$ & $3.52 \%$ \\
\hline $\begin{array}{c}\text { High fission gas release } \\
\text { from the matrix }\end{array}$ & $5.15 \%$ & $1.93 \%$ & $7.08 \%$ \\
\hline
\end{tabular}

Table 2.11: Prediction of fission gas release for Rod 2

Considering the amount of fission gas release of Rod 2, an assessment was made assuming a similar release in Rod 1 to evaluate its effect on cladding deformation. The outcomes of this assessment confirmed the validity of the fuel rod design parameters of Table 2.7 because the release would increase the cladding deformation but the burst criteria are expected not to be met.

The values estimated in Table 2.11 can be used as benchmark of the fission gas release measurements described in 3.4.3. 


\section{Bibliography}

[1] W. Wiesenack, "Overview of HRP LOCA testing”, HRP LOCA Workshop, Lyon, May 2012

[2] M. Flanagan, P. Askeljung, "Observation of fuel fragmentation, mobility and release in integral, high-burnup, fueled LOCA tests", Presented at Enlarged Halden Program Group Meeting, Sandefjord, Norway, 2-7 October 2011

[3] Patrick A.C. Raynaud, "Fuel Fragmentation, Relocation and Dispersal During the Loss-of-coolant Accident", NUREG-2121, US NRC 2012

[4] G. Lerchl, H. Austregesilo, P. Schöffel, D. von der Cron, F. Weyermann, "ATHLET Mod 3.0 Cycle A User's Manual“, GRS 2012

[5] H. Austregesilo, C. Bals, A. Hora etc, „ATHLET Mod. 3.0 Cycle A Models and Methods, GRS, November 2012

[6] "TRANSURANUS handbook", European Commission, JRC Institute for Transuranium Elements 2011

[7] K. Lassmann, H. Blank, "Modelling of fuel rod behavior and recent advances of the TRANSURANUS code", Nuclear Engineering and Design 106, 1988 (291-313

[8] H. Stehle, "Performance of oxide nuclear fuel in water-cooled power reactors", Journal of Nuclear Materials 153 3-15 1998

[9] G. Pastore, L. Luzzi, V. Di Marcello, P. Van Uffelen, "Physics-based modeling of fission gas swelling and release in $\mathrm{UO}_{2}$ applied to integral fuel rod analysis", Nuclear Engineering and Design, March 2013, 75-86

[10] S. Van den Berghe, A. Leenaers, B. Vos, L. Sannen, M. Verwerft, „Observation of a pellet-cladding bonding layer in high power fuel“, Belgian Nuclear Research Centre SCK-CEN, Belgium

[11] R.S. Nelson, J. Nucl. Mater. 31 (1969) 153

[12] W. Hering, "The KWU fission gas release model for LWR fuel rods", Journal of Nuclear Materials 114 (1983) 41-49

[13] Spino, J., et al., J Nucl Mater (2005) 346, 131

[14] C.Vitanza, "Observations on fuel fragmentation and relocation in Halden LOCA experiments", Halden LOCA Workshop, Lyon 29-30 May 2012

[15] G. Locatelli, M. Mancini, N. Todeschini, "Generation IV nuclear reactors: Current status and future prospects, Energy Policy 61 1503-1520, 2013

[16] C. Yoon, S. Deok Hong, J. M. Noh, Y. W. Kim, J. Chang, "Thermal-fluidic characteristics of a high temperature heater in an experimental helium loop for VHTR simulations", Nuclear Engineering and Design, Volume 264, pg. 257-267, November 2013

[17] G.L. Fiorini, A. Vasile, “ European Commission $-7^{\text {th }}$ Framework Programme: The collaborative Project on European Sodium Fast Reactor (CP ESFR), Nuclear Engineering and Design, Volume 241, Issue 9, pg. 3461-3469, September 2011

[18] A. Alemberti, V. Smirnov, C. F. Smith, M. Takahashi, " Overview of lead-cooled fast reactor activities", Progress in Nuclear Energy, December 2013

[19] P. Auerkari, "Mechanical and physical properties of engineering alumina ceramics", VTT Manufacturing Technology, 1996 
[20] "ATHLET Mod 3.0 Cycle A ATHLET Input Data Description", GRS, October 2012

[21] L.J. Siefkin, E.W. Coryell, E.A. Harvego, "SCDAP/RELAP5 MOD 3.3" Code Manual MATPRO - A Library of Materials Properties for Light-Water-Reactor Accident Analysis", NUREG/CR-6150, Vol.4, Rev. 2, INEL-96/0422

[22] T.Paloposki, L.Liedquist, "Steel emissivity at high temperatures", VTT RESEARCH NOTES 2299, ESPOO 2004

[23] F. Khattout, "The BWR fuel LOCA test IFA-650.13 in-pile results", HPG Meeting Halden, 30-31 October 2012

[24] Chan Bock Lee, Dae Ho Kim, Jae Seung Song, Je Gun Bang, Youn Ho Jung, „, RAPID model to predict radial burnup distribution in LWR $\cup_{2}$ fuel", Journal of Nuclear Materials 282 (2000) 196204

[25] G. Pastore, L. Luzzi, V. Di Marcello, P. Van Uffelen, "Physics-based modeling of fission gas swelling and release in $\mathrm{UO}_{2}$ applied to integral fuel rod analysis", Nuclear Engineering and Design, March 2013, 75-86

[26] P. Van Uffelen, Contribution to the Modelling of Fission Gas Release in Light Water Reactor Fuel, PhD Thesis, Nuclear engineering, University of Liege, 2002

[27] M. Adorni, A. Del Nevo, F. D’Auria, O. Mazzantini, "A procedure to Address the Fuel Rod Failures during LB-LOCA Transient in Atucha-2 NPP", Science and Technology of Nuclear Installations, 2011

[28] G. Khvostov (PSI), "Post-test analysis for LOCA test IFA-650.12 and preliminary proposal for IFA650.13", The 146th Meeting of the HPG , Lyon, France, May 31-June 1, 2012

[29] C. Vitanza, "Observations on fuel fragmentation and relocation in Halden LOCA experiments", Halden LOCA Workshop, Lyon May 2012

[30] K.Yueh, "Fuel Fragmentation, Relocation \& Dispersal Current Understanding and Test Results", NRC Public Meeting, March 2014

[31] J.B. Ainscough et al., J. Nucl. Mater. 82 (1979) 439

[32] V. Rondinella, T. Wiss, "The high burn-up structure in nuclear fuel", Materials Today, December 2010, Vol. 13(12): 24-32 


\section{Experiment execution and results}

\section{Contents}

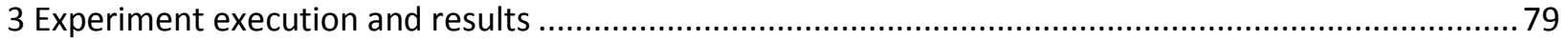

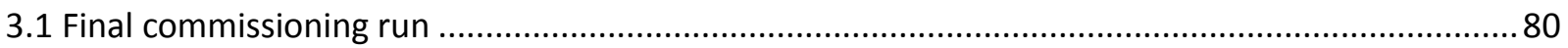

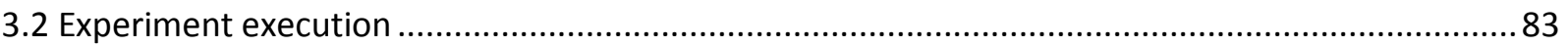

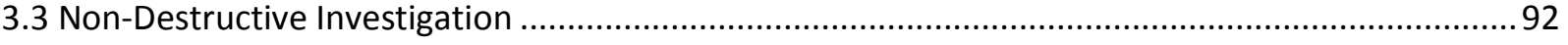

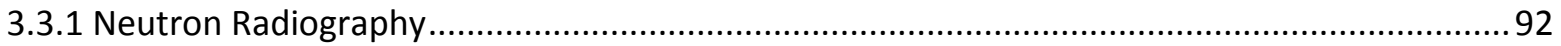

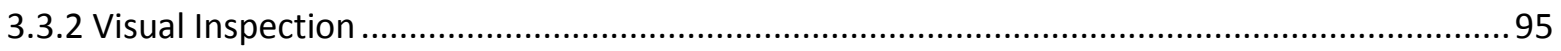

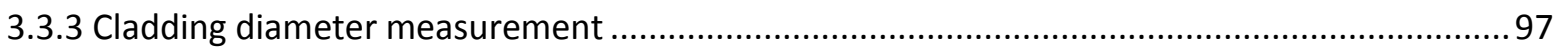

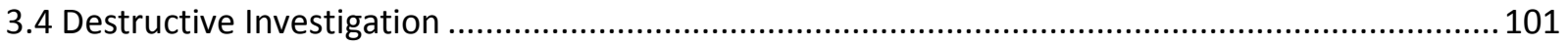

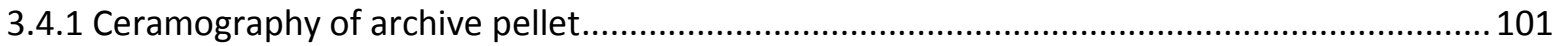

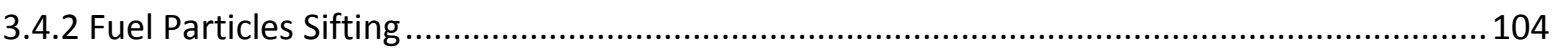

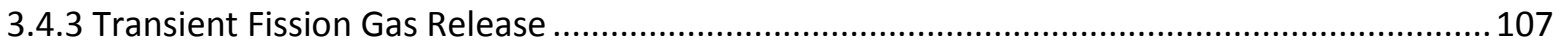

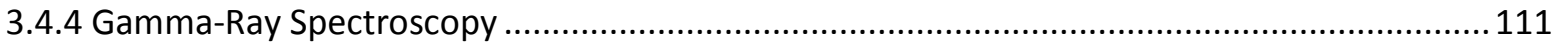

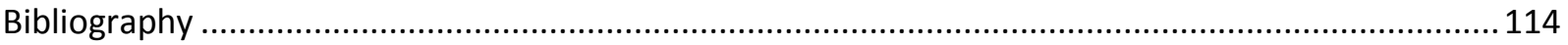

Chapter 2 contains the research background and observations and calculations tools used for the design of the experiment. After the execution of the last commissioning test for the final validation of the fuel rod design parameters and experimental set up as described in 3.1, the experiment was executed. The remanufacturing of the irradiated fuel rods, preparatory phases of the experiment and its execution are described in 3.2.

The post-experiment investigations are described in 3.3 (non-destructive investigations) and 3.4 (destructive investigations). The non-destructive investigations included for the three rods the neutron- 
radiography, the visual inspection and the axial cladding diameter measurements to determine the cladding deformation during the transient. The destructive investigations included the ceramography of an archive pellet not subject to the LOCA test, the fuel sifting of Rod 1 and Rod 2 to determine the fragment size distribution and the analysis of the fission gas release of the three rods during the transient in terms of integral amount of gas released and gamma spectrum of the three gas samples.

\subsection{Final commissioning run}

The final commissioning run was performed to validate and confirm the experimental set up and the rod design parameters calculated with the ATHLET-TRANSURANUS methodology of 2.2.3. Only the behavior of Rod 1 and Rod 2 was tested, because the behavior of Rod 3 was considered well predictable. These last commissioning runs were the final mock-up of the test, with the foreseen equipment, instrumentation, test rig and test's procedures. The only differences were the fuel rod's materials used: fresh Zircalloy- 4 as a cladding and alumina pellets $\left(\mathrm{Al}_{2} \mathrm{O}_{3}\right)$ as fuel. Alumina pellets were selected because alumina is a ceramic material easy to be procured and to be remanufactured. The difference in properties between $\mathrm{UO}_{2}$ and $\mathrm{Al}_{2} \mathrm{O}_{3}$ are shown in Table 3.1. The fuel rod design parameters to be validated are the ones shown in Table 2.7.

\begin{tabular}{|l|l|l|}
\hline Parameter at normal LWR condition & $\mathrm{Al}_{2} \mathrm{O}_{3}[1]$ & $\mathrm{UO}_{2}[2]$ \\
\hline Density $\left[\mathrm{kg} / \mathrm{m}^{3}\right]$ & 3,900 & 10,500 \\
\hline Thermal Conductivity $[\mathrm{W} /(\mathrm{mK})]$ & 18 & 3.0 \\
\hline Heat Capacity $(\mathrm{J} / \mathrm{kg} \mathrm{C})$ & 1100 & 410 \\
\hline
\end{tabular}

Table 3.1: Comparison of properties uranium dioxide vs. alumina

A first commissioning test was run for Rod 1 having the other 2 capsules in the test rig as dummies and at a later stage, a second commissioning test was run for Rod 2.

The instrumentation installed on the test rig was the following:

- Rod 1: two thermocouples on the capsule in correspondence of the middle and top part of the active fuel and a capsule pressure transducer.

- Rod 2: three thermocouples on the capsule in correspondence to the bottom, middle and top part of the active fuel and a capsule pressure transducer.

Figure 3.1 and 3.3 show the transient results of the two commissioning tests. The main observations can be summarized as following:

- Rod 1: Observing the pressure transient, no burst was detected: the pressure of the capsule increases following the heating up, but then it stabilizes with the capsule approaching the maximum temperature. The visual inspection that followed the test (Figure 3.2) showed a cladding deformation along the entire axial length with a noticeable deformation at the top of the fuel. This was caused by a faster heating up of the upper plenum structure. After noticing 
this phenomenon, a thermal insulation was installed on the top and bottom of outer side of the capsule in correspondence to the fuel bottom and end plugs.

- Rod 2: During the test execution, some issues were noticed with the pressure transducer device (possible leakage). Consequently, the increase of pressure due to the heating up of the capsule was not detected. However, at about $740 \mathrm{~s}$, the burst effect is shown with the sudden linear increase of pressure. The burst effect is usually expected as a sudden pressure drop of the capsule's pressure, but the possible leakage filtered the signal's discontinuity. The visual inspection of Figure 3.4 shows a pronounced ballooning on the mid-bottom part of the fuel that corresponds also to the burst location.
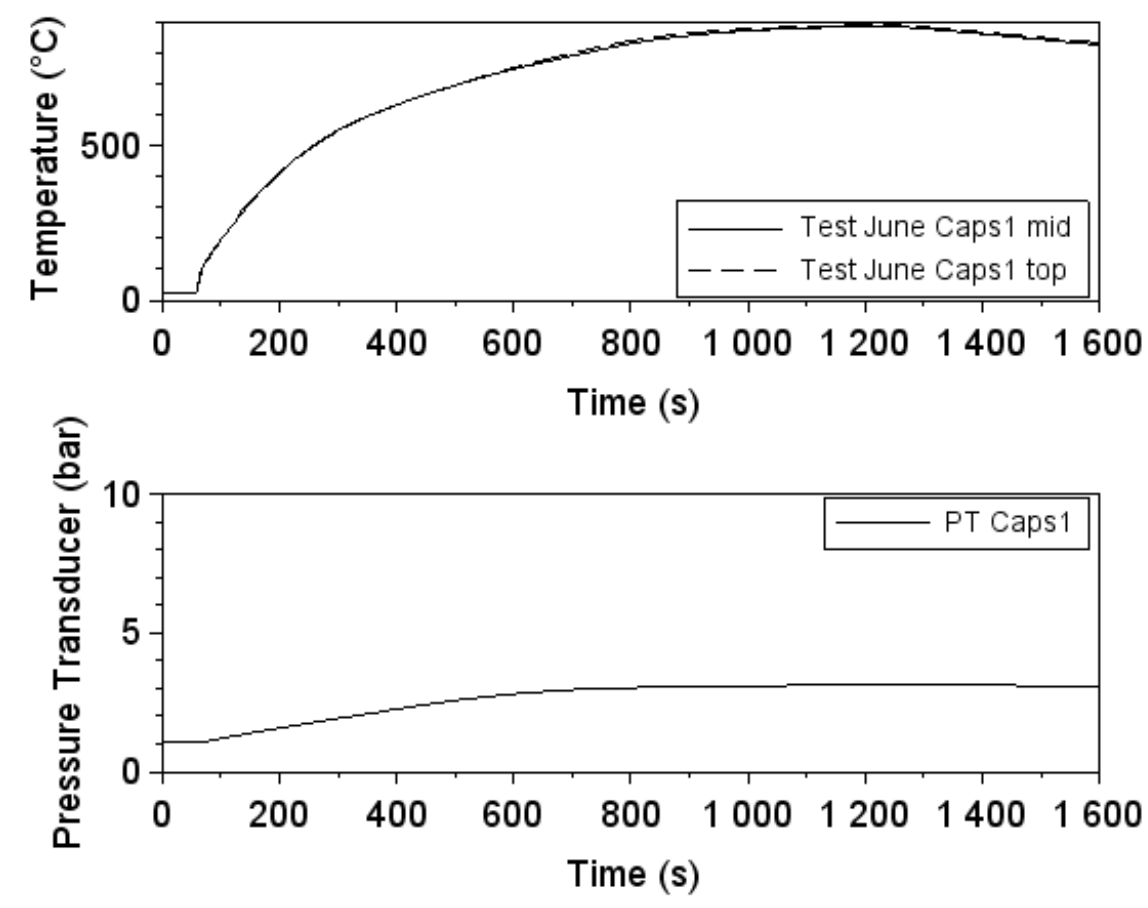

Figure 3.1: Final commissioning run for Rod 1

Top Figure: capsule temperature; Bottom Figure: capsule pressure

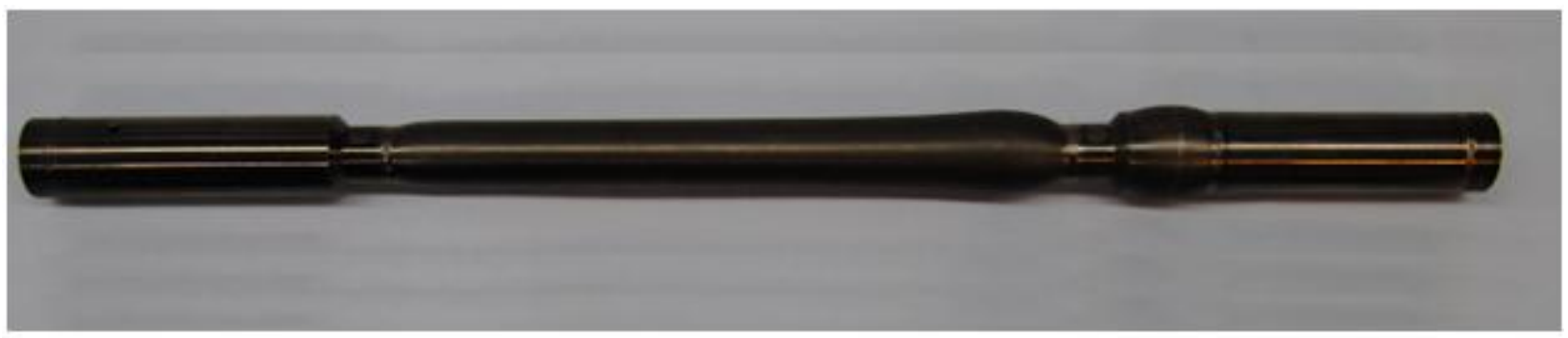

Figure 3.2: Visual inspection of Rod 1 after final commissioning run 

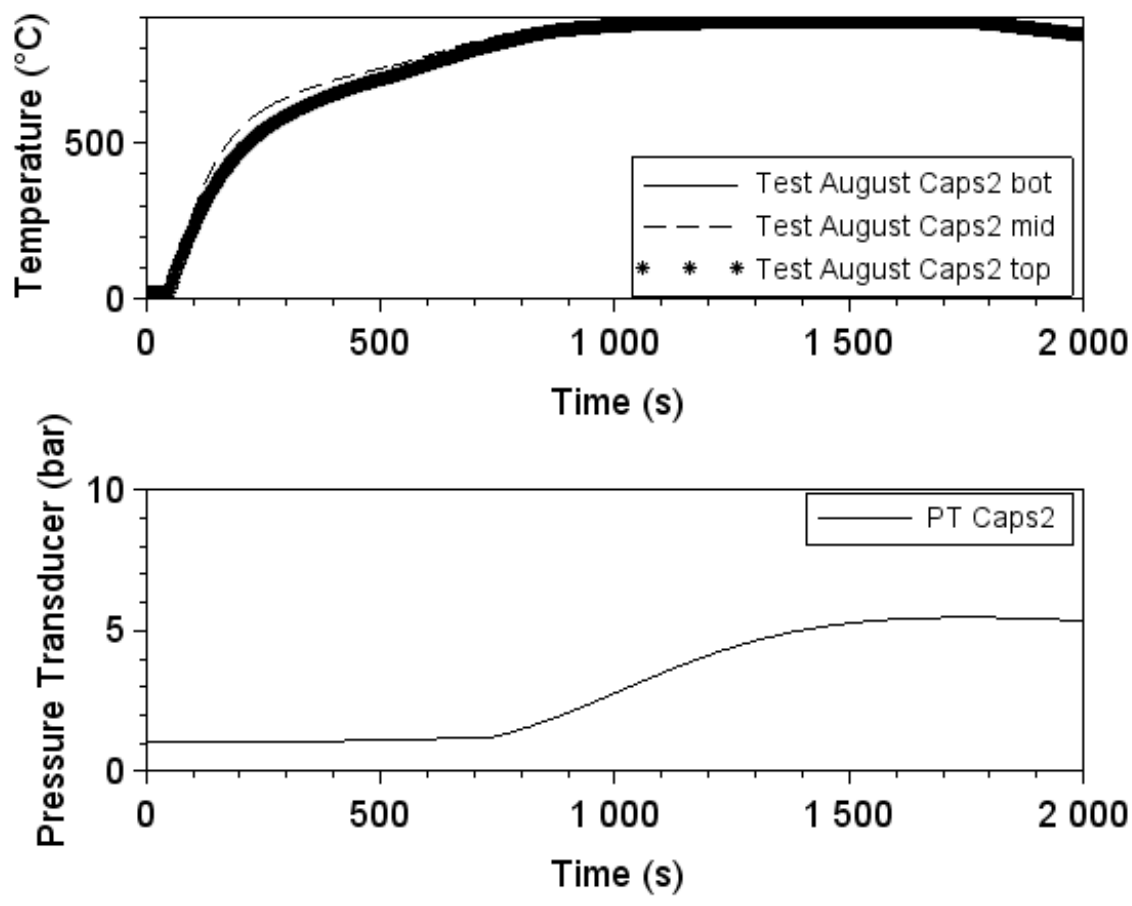

Figure 3.3: Final commissioning run for Rod 2

Top Figure: capsule temperature; Bottom Figure: capsule pressure

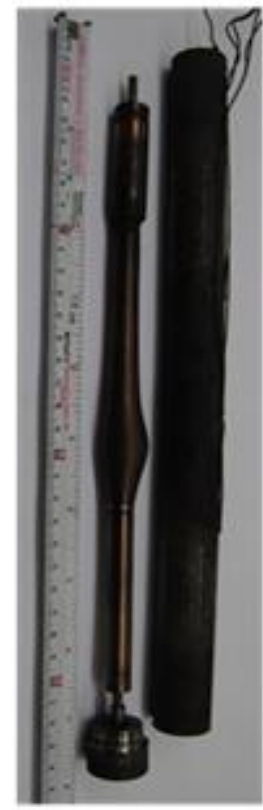

a)

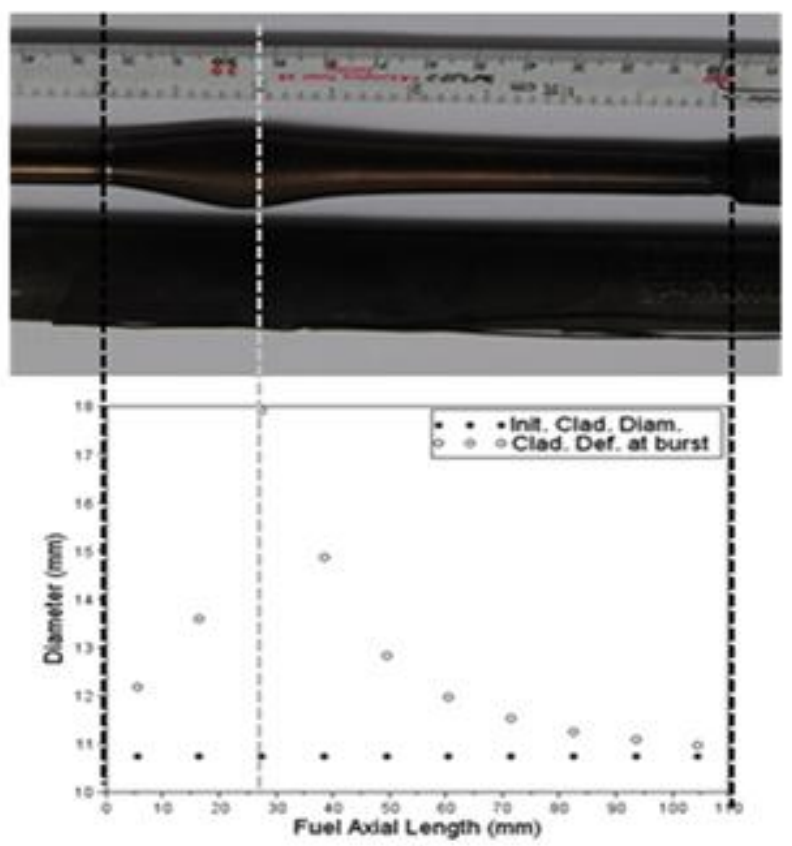

b)

Figure 3.4: Visual inspection of Rod 2 after final commissioning run a) visual Inspection; b) benchmark with TRANSURANUS calculation 
Figure 3.4 b) shows the benchmark between the commissioning results and the TRANSURANUS calculation in terms of cladding deformation. A rigorous validation would have implied an axial diameter measurement of the cladding to determine the cladding's diameter at different axial location. However, such a detailed validation was only done for the real test as shown in 3.3.3. In terms of burst time, Table 2.6 offers burst time sensitivities based on different temperature peak profiles and fill-in gas pressure: for a fill-in gas pressure of 25 bar, the burst time is expected between 715 and $740 \mathrm{~s}$, a time window where the increase of pressure as shown in Figure 3.3 occurs, when considering the start of the transient at the beginning of the heating up (at circa $50 \mathrm{~s}$ ).

The commissioning tests performed on Rod 1 and Rod 2 confirmed the expected behaviors of the two rods: Rod 1) ballooning without burst and Rod 2) ballooning with burst. Moreover, TRANSURANUS predicted with a good level of accuracy the burst time and the cladding deformation (Table 2.6). It should be underlined that the commissioning test calculations were made using the properties of alumina as fuel pellets in the ATHLET run.

To prove that the fuel rod design parameters were appropriate also with irradiated fuel rods, updated calculations have been made with ATHLET and TRANSURANUS to consider the properties of high burnup fuel for the cladding and the pellets: ATHLET updates the fuel properties with the input option BURNUP [3], while TRANSURANUS [4] considers in details the power history of the fuel rod as shown in 2.3.1.

\subsection{Experiment execution}

A successful execution of the commissioning runs was the green light for the starting of the remanufacturing of the irradiated rods. This operation was done inside the hot cells of the Halden Reactor Project in Kjeller [5]. The main irradiated fuel segment has been cut to obtain the three rod segments. These segments have been welded to the top and bottom structures shown in Figure 2.15 through circumferential weld through TIG-welding methodology [6]. At a later stage, the upper plenum of the three rods has been filled with Helium at the pressure as shown in Table 2.7. This was possible because of a vent hole left on the upper structure that was plugged immediately after the filling operation through TIG-welding, when the inner and outer system sealed environments were still under pressure.

Figure 3.5 shows details of the electric oven (left) and of the experimental set up (right) in the HRP workshop outside the hot cell. The left figure a) allows capturing details of the oven: the oven is heated by vertical winding heater in an $\mathrm{Al}_{2} \mathrm{O}_{3}$ insulation shell. The test rig with the three capsules is located in the centre of the oven inside a quartz tube necessary to fix its position radially and vertically. It should be underlined that at this stage, the three capsules did not contain the three irradiated fuel rod segments that were manufactured and stored in the hot cell. The bottom and top of the quartz tube were plugged with insulation materials to mitigate the axial heat losses. The top insulation material allowed wires connection between the instrumentation devices and data acquisition system. 
Three dimensional details of the experimental rig are provided in Figure 3.6. The thermocouples welded on the capsules were type $\mathrm{K}$ (chromel-alumel) that are suitable for operating temperatures of $900^{\circ} \mathrm{C}$ [7].

The Piping and Instrumentation Diagram (P\&ID) and the manufactured rods' pressure transducer system are shown in Figure 3.7. The system was conceived to accomplish three different functions:

- Create vacuum in the three capsules and fill them with helium to reach a pressure of 1 bar at Room Temperature.

- During the test monitor the capsules' pressure through a pressure transducer device for each capsule. The pressure transducer allowed detecting burst from a rod because the pressure in the capsule suddenly increases.

- After the test, gas from each of the three systems was collected. Of particular interest is the gas from the capsule of Rod 2. This rod is expected to burst and consequently the capsule's environment after the test was filled with the fission gas release from the rod's $\mathrm{UO}_{2}$ matrix during the LOCA transient. This gas was at a later stage used for the investigations described in 3.4.3 and 3.4.4.

The oven, the three capsules and the pressure transducer piping system were moved inside the hot cells. A wire connection exists between the inside and outside of the hot cells. Each irradiated rod segment was installed inside each capsule and the capsule connected to the pressure transducer system as described above.

The test started setting up the target temperature of the oven to $900^{\circ} \mathrm{C}$. When the oven's temperature stabilized, the test-rig was dropped in the oven through the quartz tube.

Figure 3.8 - Figure 3.11 show the data recorded during the test: the temperature recorded by the midtop and mid-bottom capsule thermocouples and the pressure measured by the pressure transducers for the three capsules. The relevant time points are listed below:

- $50 \mathrm{~s}$ : The test rig with the three capsules is dropped in the oven and the temperature of the capsules started to increase instantaneously. The increase of temperature of the capsules is followed by their increase of pressure.

- $729 \mathrm{~s}$ : A sudden increase of pressure is detected for Capsule 2 from 2.85 bar to 5.95 bar that corresponds to a burst of Rod 2. The temperature of the capsule at this time point is about 843 ${ }^{\circ} \mathrm{C}$.

- $\quad 1000 \mathrm{~s}$ : The three capsules reached a condition of equilibrium: the capsules' temperature is about $890^{\circ} \mathrm{C}$ and the pressure of the three capsules is: Capsule of Rod 1: 2.64 bar, Capsule of Rod 2: 6.10 bar and Capsule of Rod 3: 2.51 bar.

- $1510 \mathrm{~s}$ : The oven is turned off and the temperature starts to slightly decrease.

The experiment was terminated with the extraction of the test rig from the oven that occurred with the oven temperature of about $600{ }^{\circ} \mathrm{C}$. 
The data recording provides also the information that Rod 1 ballooned as expected. The maximum pressure of Capsule 1 was slightly higher than the maximum pressure of Capsule 3 . Since the capsule and pressure transducer systems did not vary substantially, this larger maximum pressure is an indication of fuel rod ballooning and consequently of reduction of volume for Capsule 1. A very preliminary calculation shows that this larger increase of pressure corresponds to approximately an average cladding radial deformation of $2.2 \mathrm{~mm}$.

Figure $3.12,3.13,3.14$ and 3.15 show the experiment execution in the hot cell and the hot cell laboratory environment with the data acquisition system. 


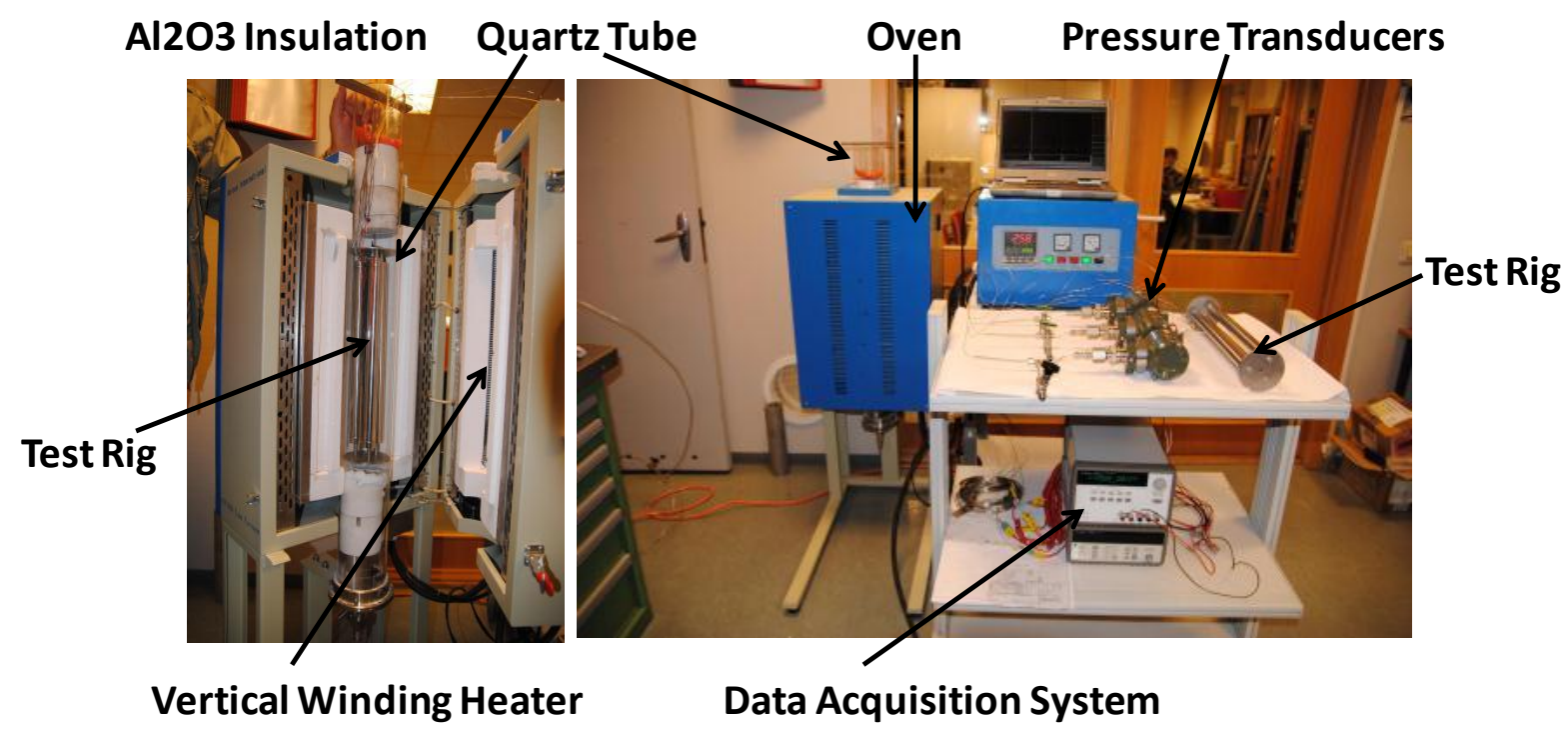

Figure 3.5: Details of the experimental set up and instrumentations Left: details of the electric oven; Right: details of the instrumentations and data acquisition system

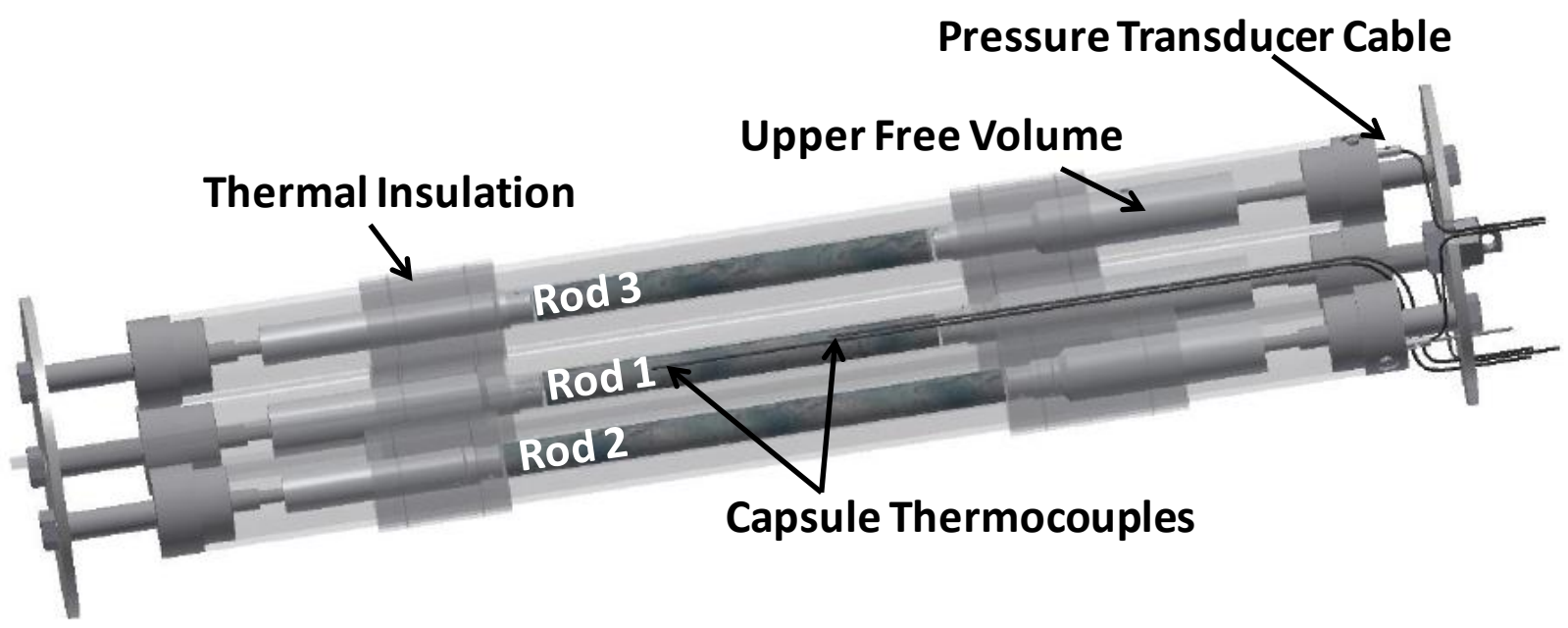

Figure 3.6: 3-D details of the experimental rig

Thermocouples locations are $25 \mathrm{~mm}$ under and above the middle of the fuel rod's location 

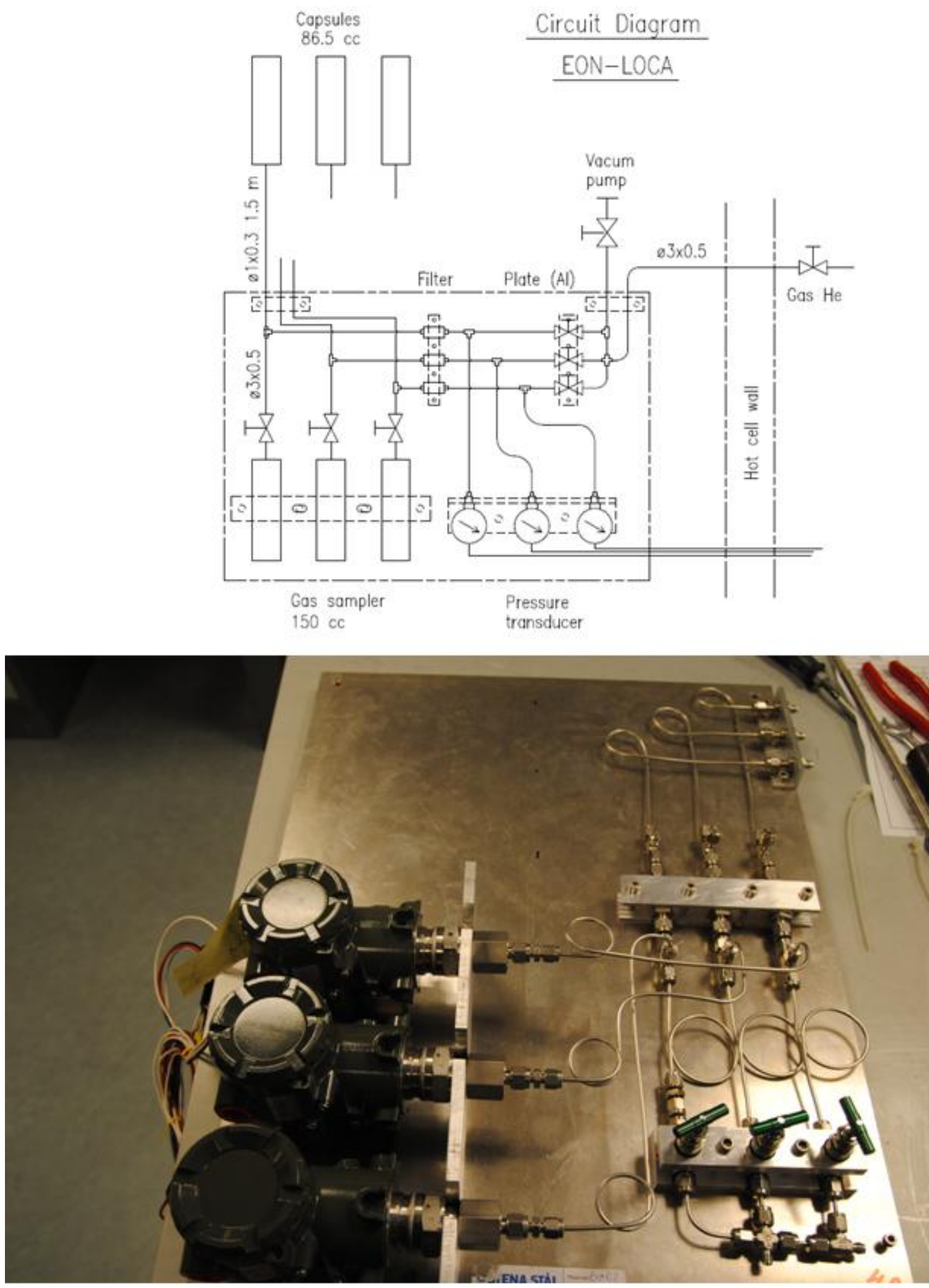

Figure 3.7: Pressure transducer piping system P\&ID of the system (top); Picture of the system outside the hot cells (bottom) 


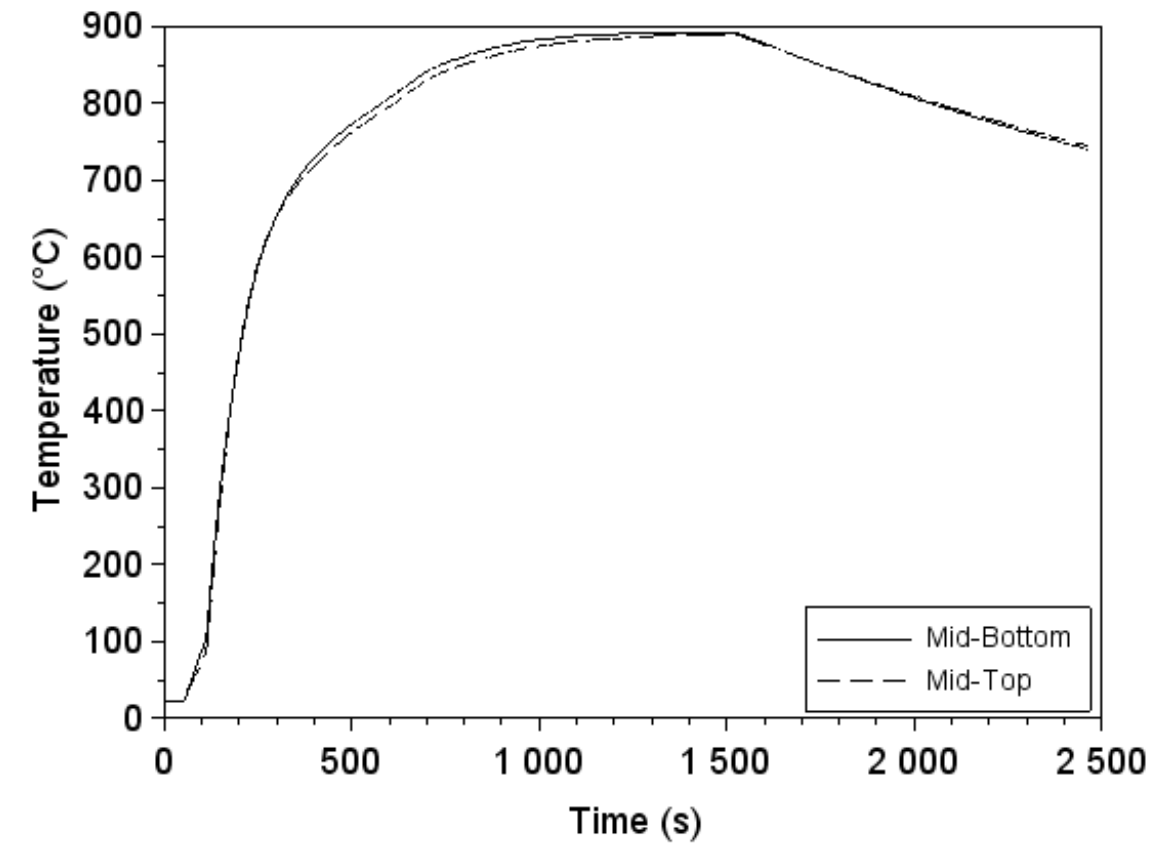

Figure 3.8: Thermocouples data recording

a) Average value of capsule bottom and top thermocouples; b) inner pressure of capsule 1, capsule 2 and capsule 3

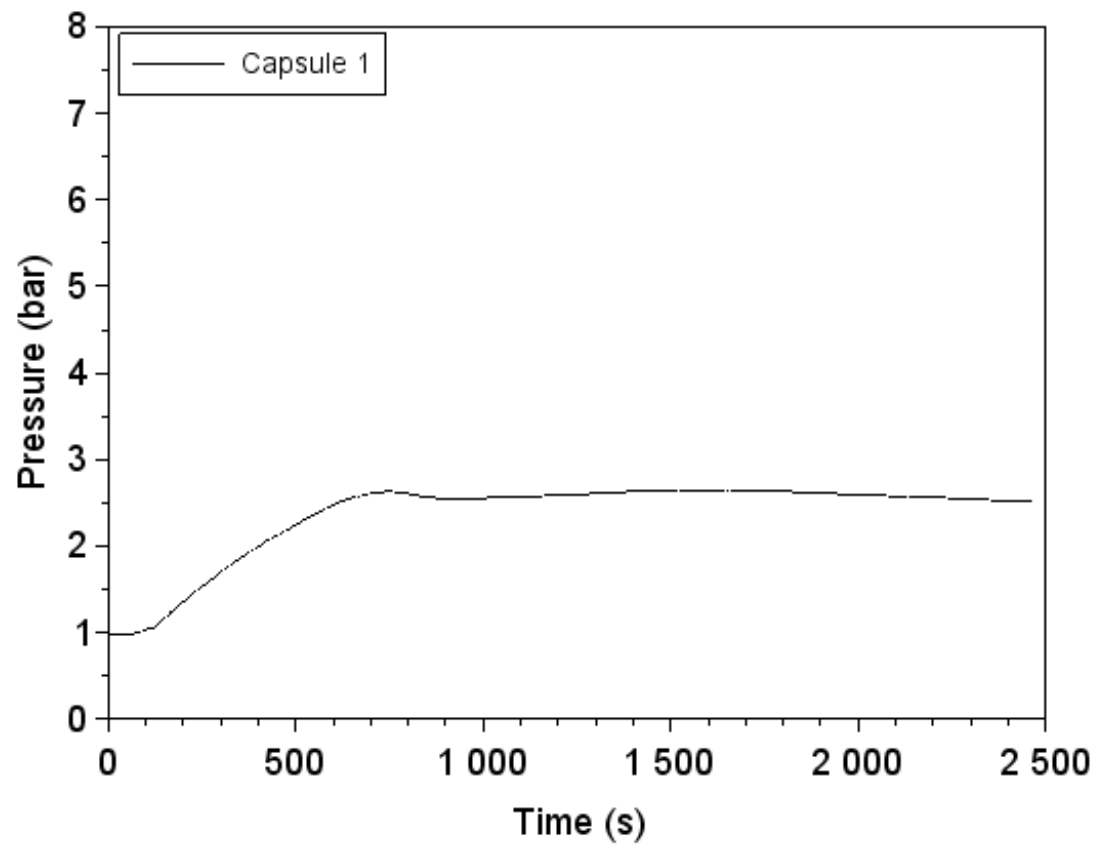

Figure 3.9: Pressure transducer data recording capsule 1 


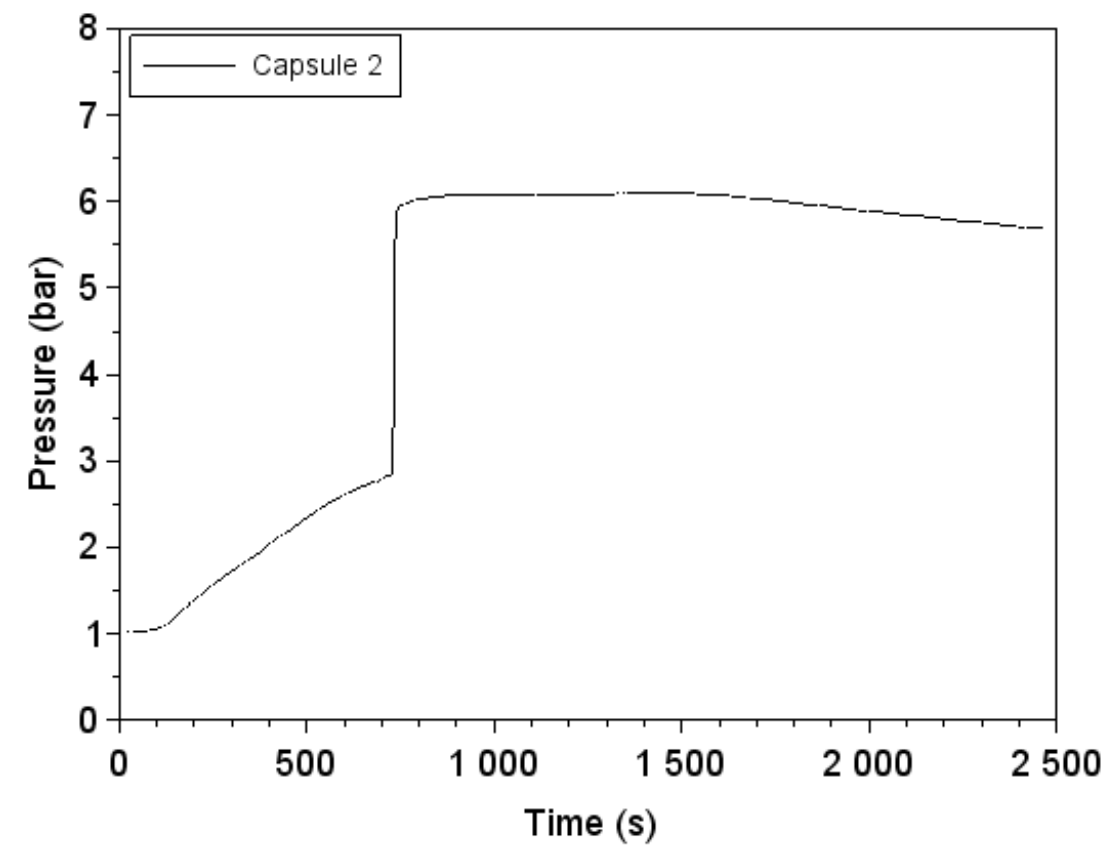

Figure 3.10: Pressure transducer data recording capsule 2

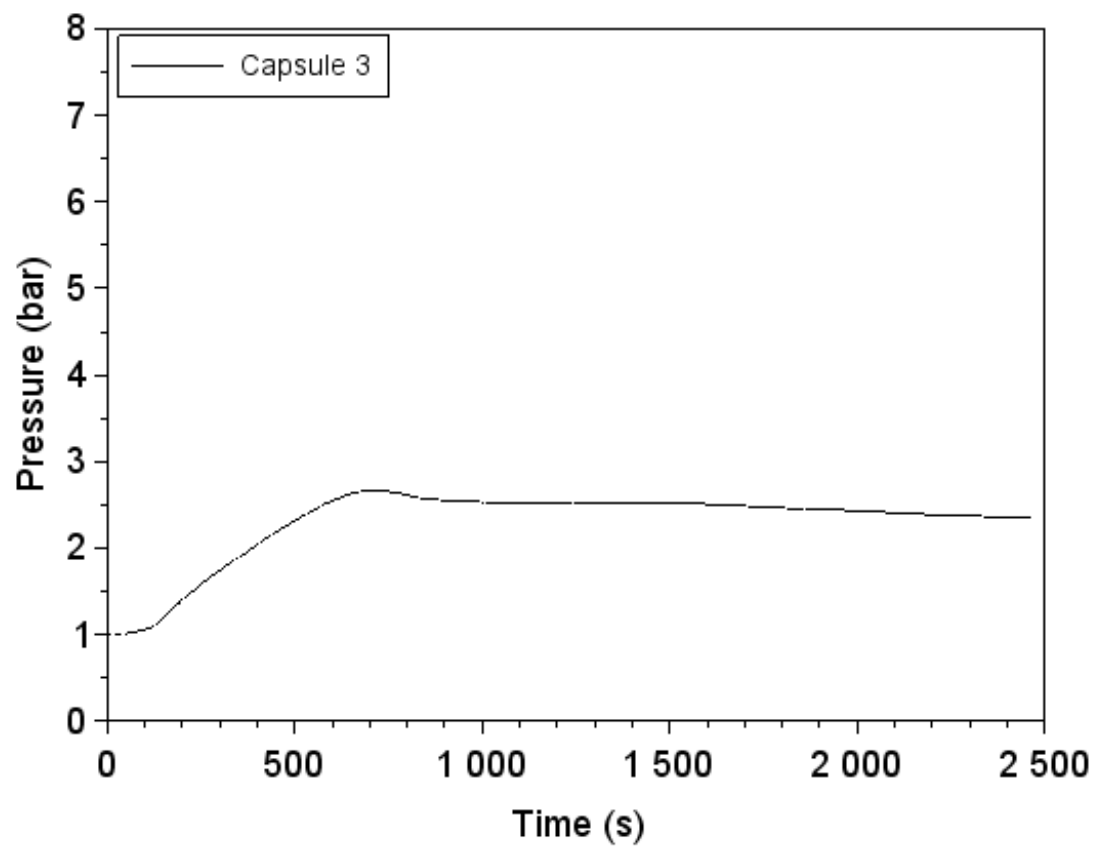

Figure 3.11: Pressure transducer data recording capsule 3 


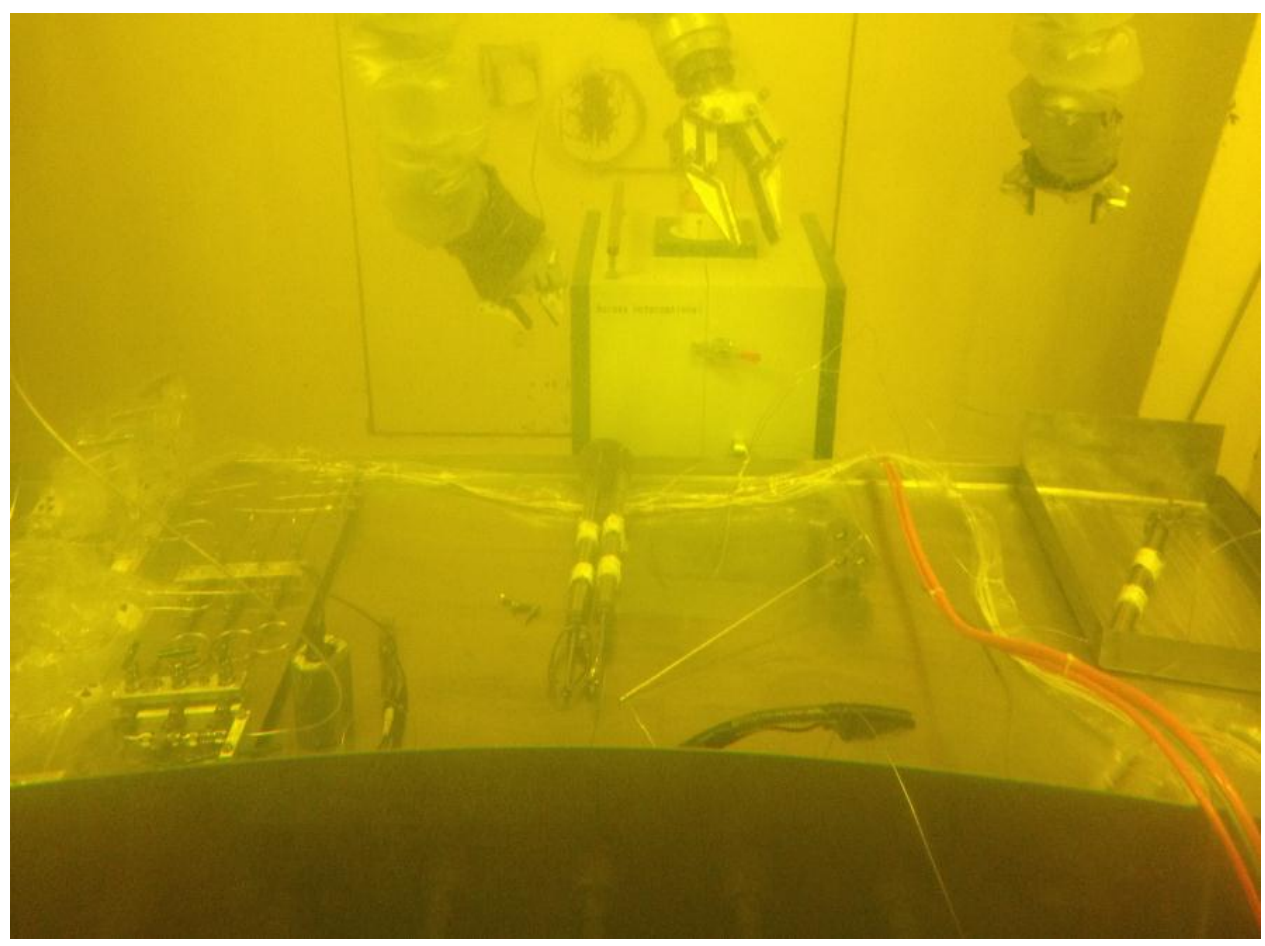

Figure 3.12: Preparatory phase of the experiment execution in the hot cells The test-rig is visible on the hot cells working table

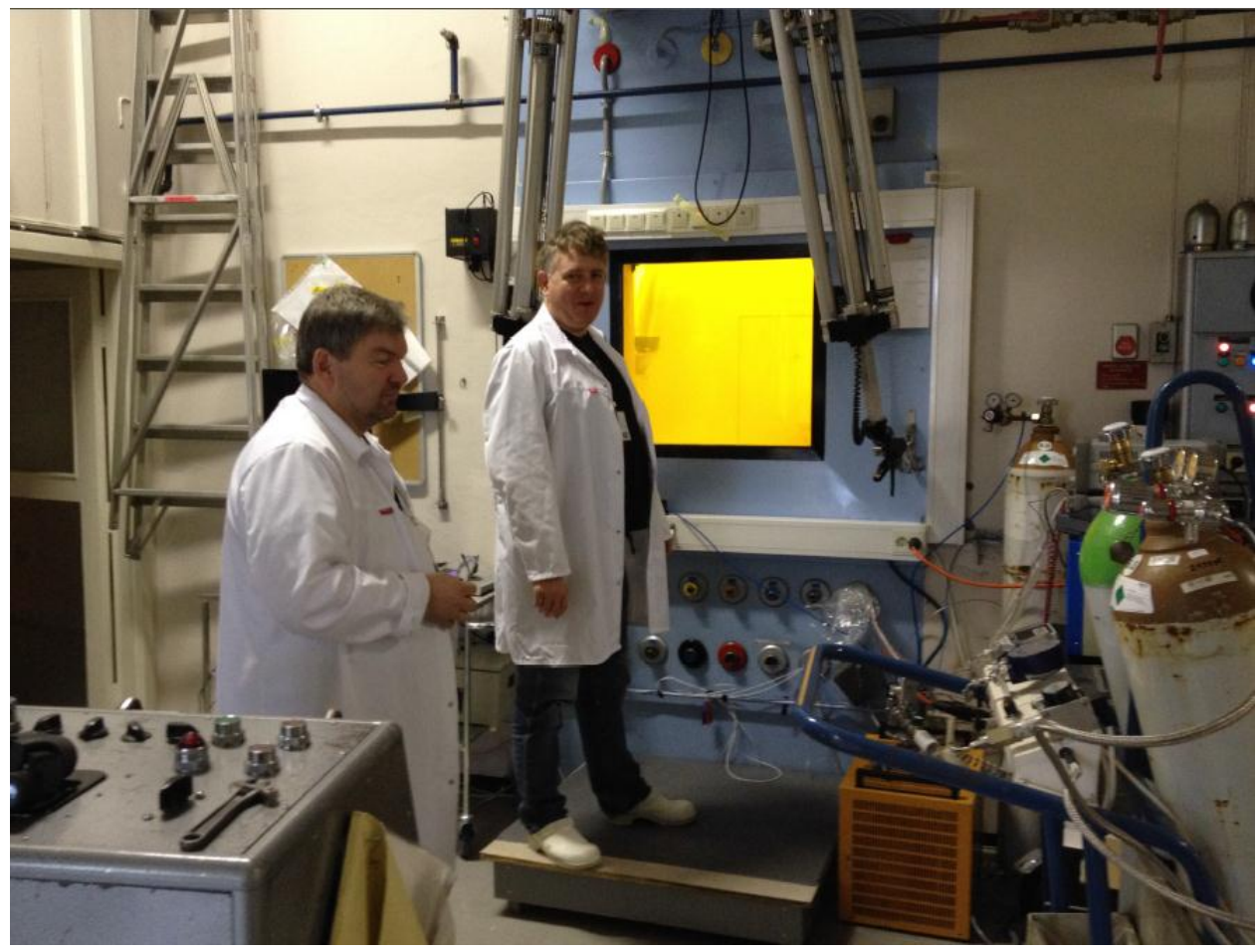

Figure 3.13: HRP Hot Cell Operators 


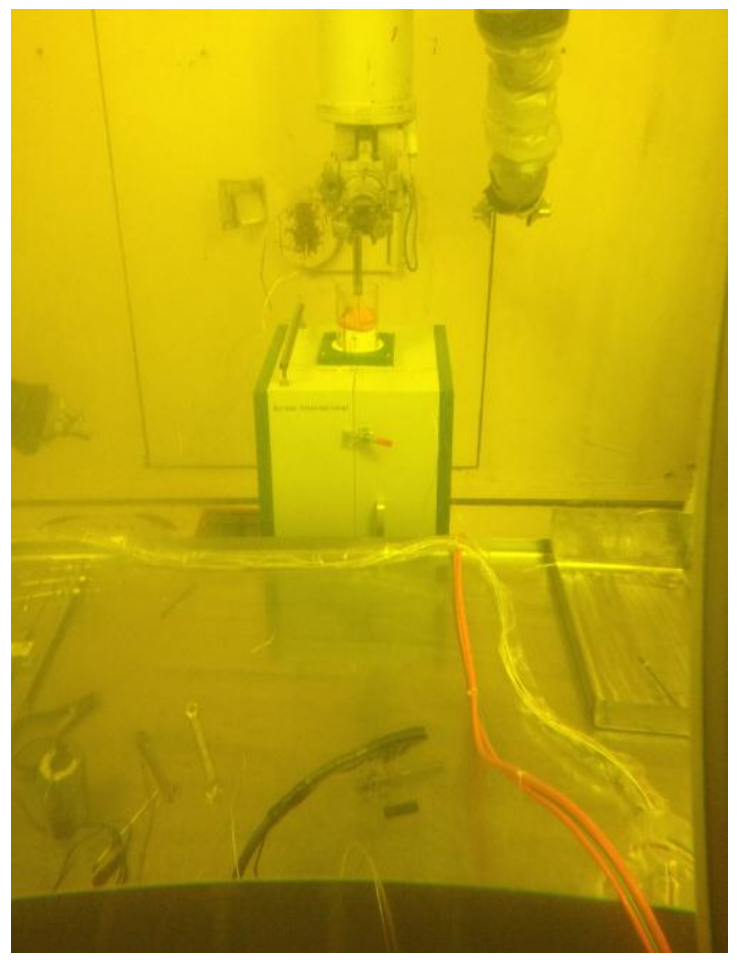

Figure 3.14: Experiment execution in the hot cells The test-rig was dropped in the electric oven

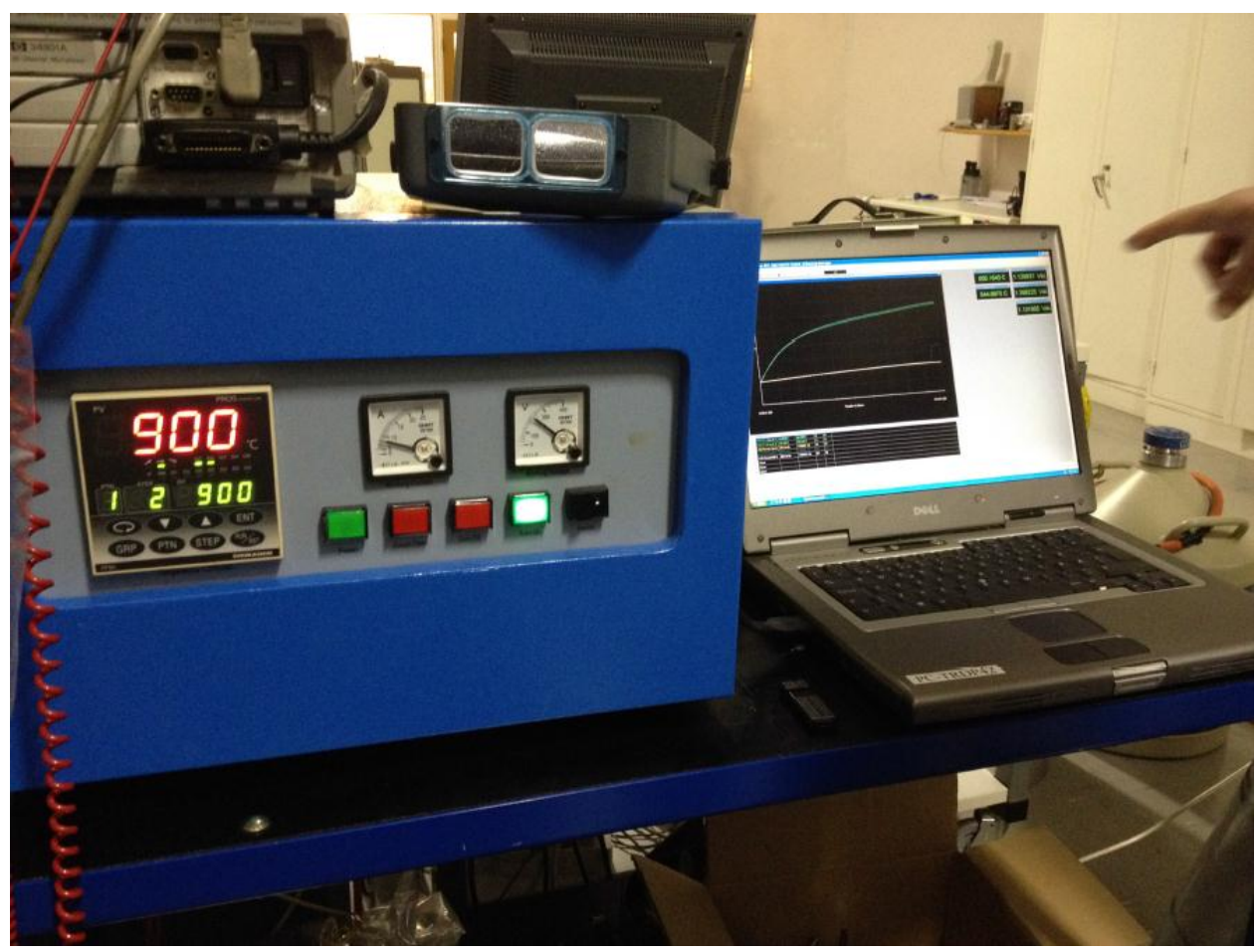

Figure 3.15: Data acquisition system during the experiment Electric oven at the target temperature of $900^{\circ} \mathrm{C}$ 


\subsection{Non-Destructive Investigation}

The first non-destructive investigation performed was the neutron radiography. It was executed after the test when the capsules cooled down and their transport to the near Jeep II Reactor was possible. At a later stage, the capsules have been brought back to the hot cell laboratory and dismantled to perform the rods' visual inspections. As last step of non-destructive investigations, the fuel rod diameter measurement was done to allow determining the cladding deformation of the three rod segments along their axial length with a high level of accuracy.

\subsubsection{Neutron Radiography}

The neutron radiography was executed at the Jeep II reactor of the IFE laboratories in Kjeller, Norway. The distance between the hot cells and the reactor is less than $500 \mathrm{~m}$. The transport operation included some movement with the capsules also in the horizontal direction.

Neutron radiography is a powerful non-destructive material examination technique and has been extensively used for image acquisition and qualitative analysis of fuel rods [8]. Example of fields of investigations on the fuel rod examinations are: cladding hydrogen up-take, reactor power ramp experiment and fuel rod degradation experiments. The neutron radiography uses the principle of attenuation of neutrons in hydrogenous materials with a high penetration for heavy metals. Because of these characteristics, neutron radiography is a complementary technique to X-ray imaging. Unlike X-rays and $\gamma$-rays, neutron interaction is characterized by nuclear rather than electronics of the medium through which it passes. The neutron radiography techniques used at the Halden Reactor Projects are Dysposium foil and X-ray film technique and Cellulose nitrate film technique with track-etch recorder [19].

The primary source of neutrons was obtained from one of the radial channels of the JEEP II Reactor [5]. The fuel rod sample was irradiated by a collimated thermal neutron beam of height $220 \mathrm{~mm}$ and width limited to $30 \mathrm{~mm}$. The thermal neutron flux in the beam channel is $\sim 10^{7} \mathrm{~cm}^{-2} \mathrm{~s}^{-1}$ and the length between graphite collimator and activation screen is circa $3000 \mathrm{~mm}$.

The neutron radiography results are shown in Figure 3.16, 3.17 and 3.18 and their main outcomes are:

- $\quad$ Rod 1: The rod ballooned for the entire axial length. The cladding ballooned as the temperature profile of the oven was relatively flat. The neutron radiography indicated a large cladding strain for all the pellets with a consequent complete removal of the cladding's geometrical constraint. The fuel pellets slightly moved radially and no fuel fragmentation can be detected, but fuel cracks along the entire axial length and powder at the bottom of the rod. There is no evidence of fuel relocation within the rod.

- $\quad$ Rod 2: The rod ballooning and burst were likely to have occurred at a bottom axial location. The cladding ballooning did not evolve along the entire axial length of the cladding but it is limited to 
the burst region. Consequently, the pellet-cladding bonding seems intact for the top 4 pellets. Fuel fragmentation occurred for 3 pellets in correspondence to the burst opening. Away from the burst region, only cracks are visible. The top 4 pellets seem intact. Fuel relocation occurred and it filled the fuel-cladding gap of the second and third pellet from the bottom of the rod.

- Rod 3: There is no evidence of cladding deformation and/ fuel fragmentation and relocation.

It should be underlined that the resolution of the neutron radiography is about $40 \mu \mathrm{m}$, and seemingly intact pellets seen on neutron radiography can have cracks.

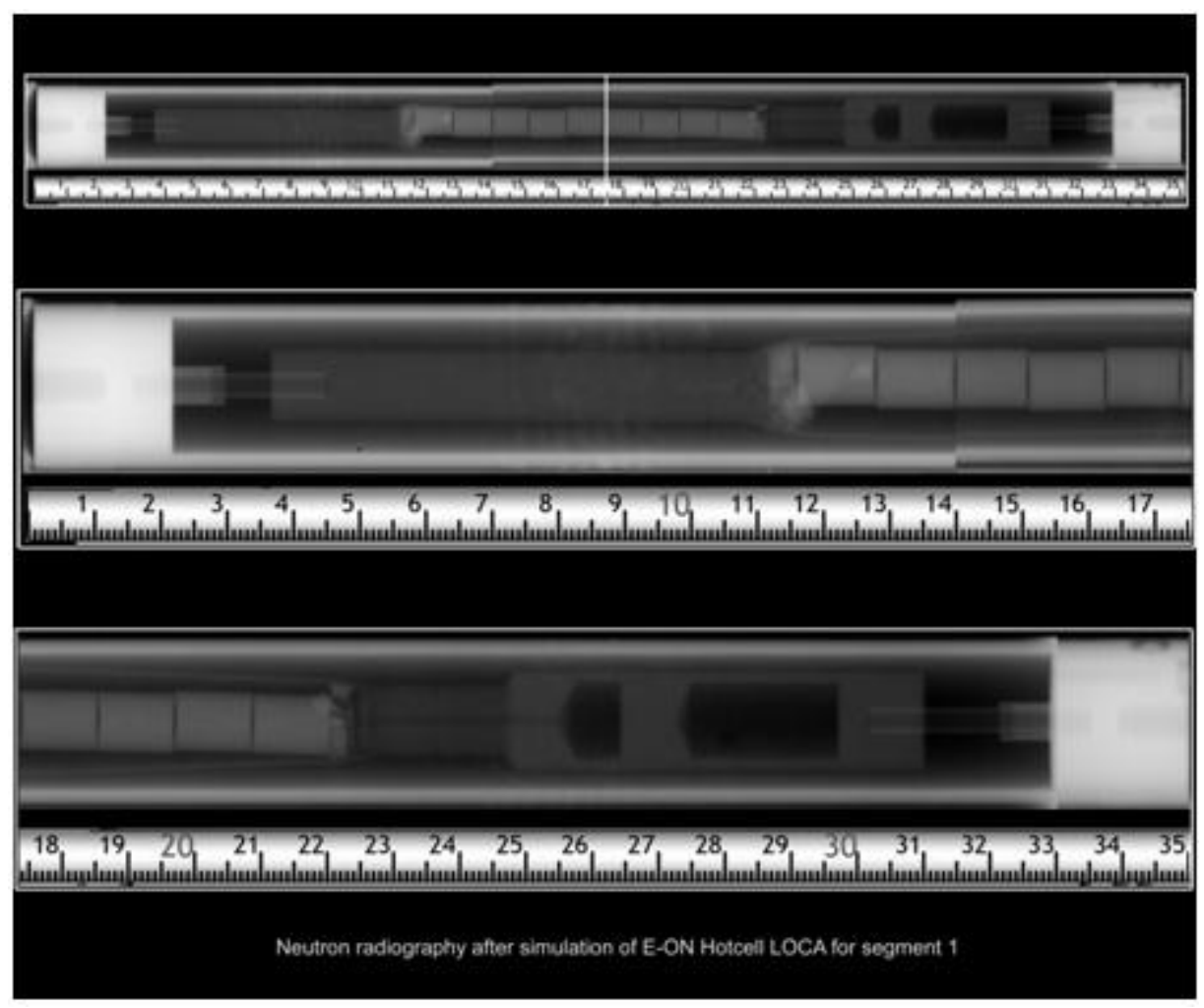

Figure 3.16: Neutron radiography Rod 1 


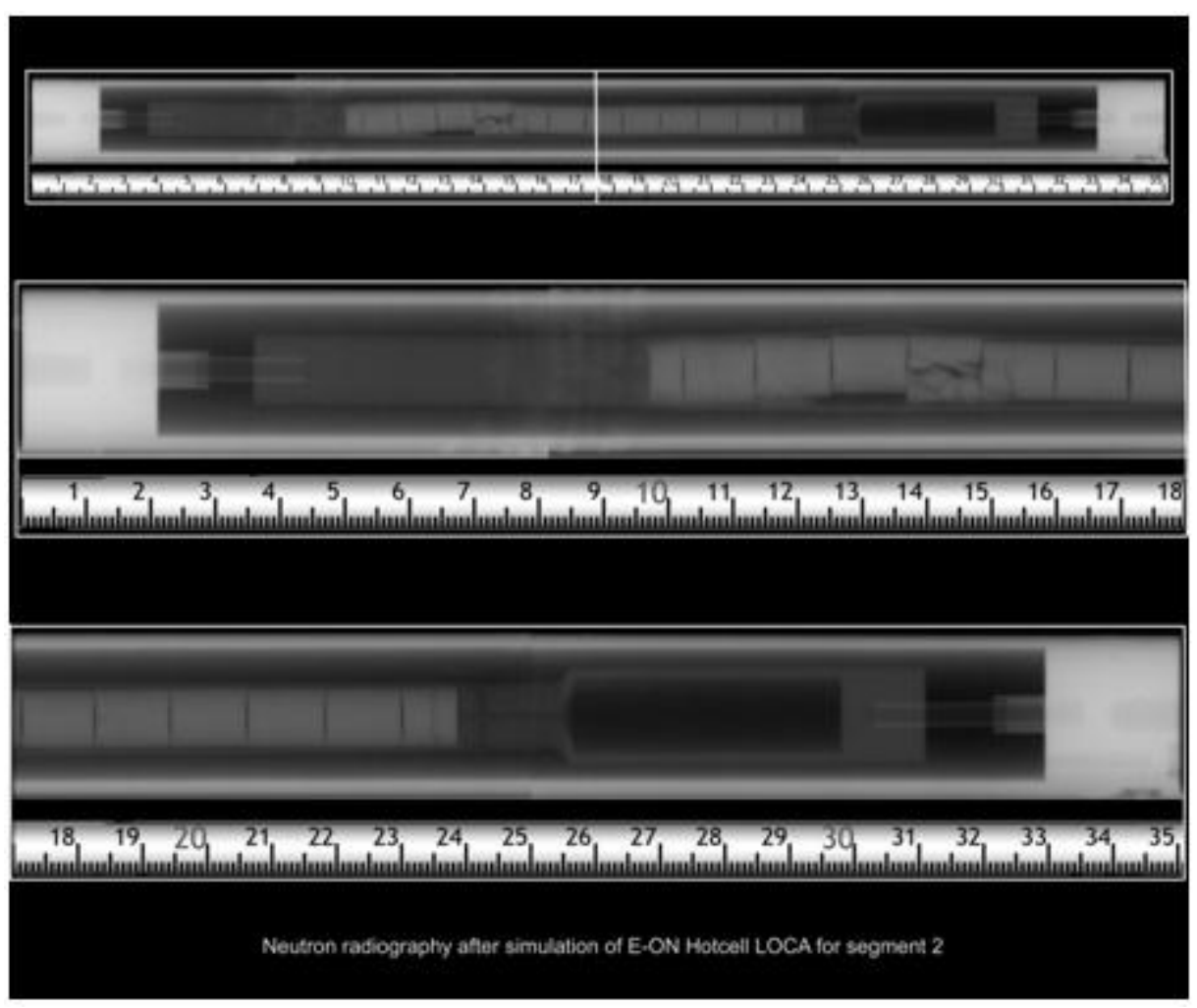

Figure 3.17: Neutron radiography Rod 2

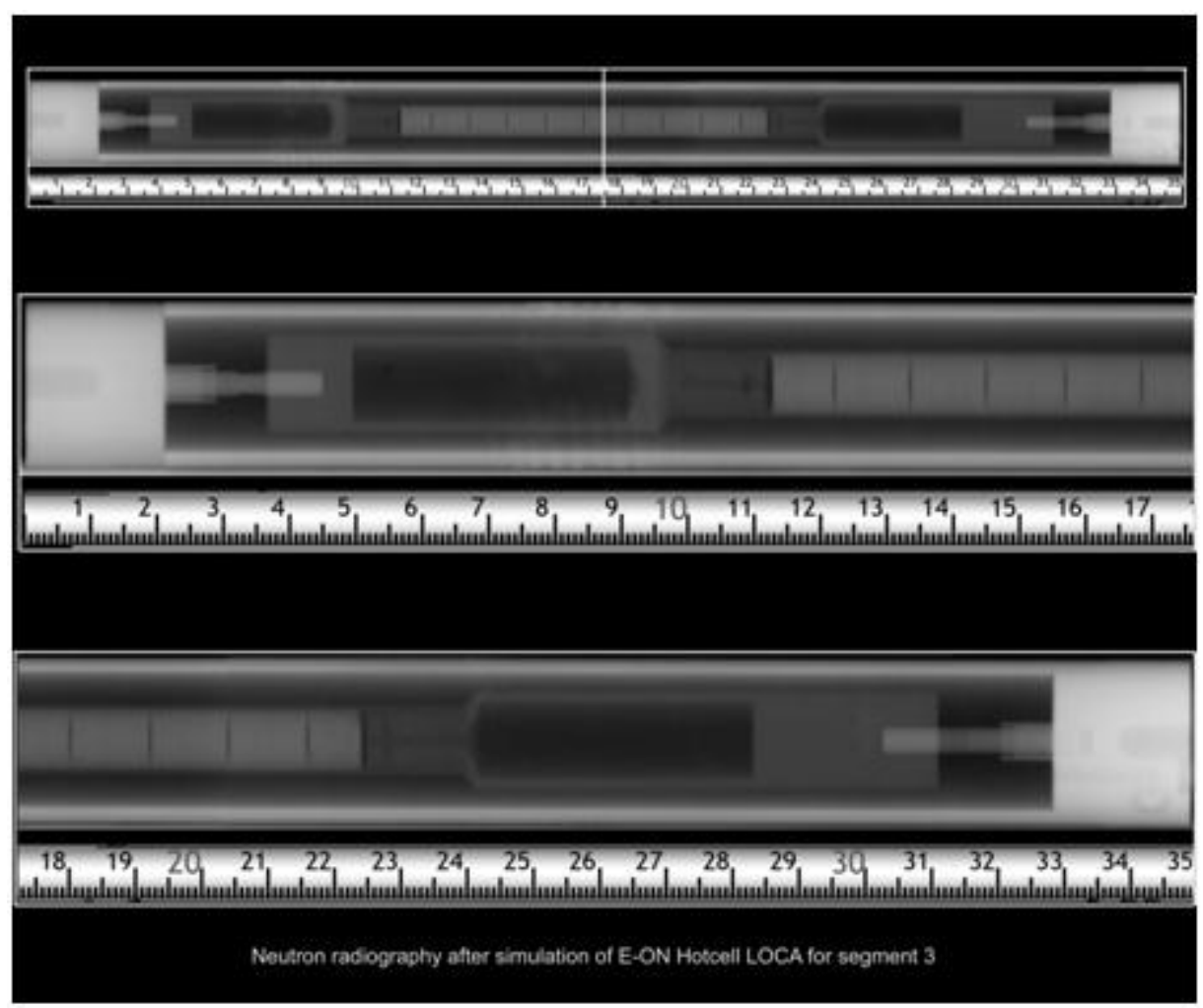

Figure 3.18: Neutron radiography Rod 3 


\subsubsection{Visual Inspection}

The visual inspection shows the status of the three irradiated segments after the test and the results are shown in Figure 3.19-3.25.

For the three segments, it is possible to observe a dark corrosion layer. It should be underlined that since the fuel rods were heated up in a Helium environment, the cladding did not experience the steam Zircaloy reaction characteristic of a LOCA [9]. Consequently, the origin of the oxidation layer is the irradiation in the commercial nuclear power plant. White spots on the black cladding outer diameter oxide as a result of cladding strain and oxide cracking were observed all over the active fuel stack for Rod 1 and Rod 2.

The visual inspection for Rod 1 at 0 and 90 degree orientation shows that the cladding deformation/ballooning occurred azimuthally symmetrically and it confirmed what was investigated during the commissioning runs of 2.2.2, where the azimuthal distribution of temperature was found to be uniform. The ballooning occurred along the entire length of the active fuel.

The visual inspection for Rod 2 at 0 and 90 degree orientation confirms that cladding deformation/strain resulted in a maximum cladding strain with burst at about $4.5 \mathrm{~mm}$ from the bottom of the active fuel. Here, the cladding formed a short and narrow burst opening. The top part of the fuel seems not having experienced relevant cladding deformation. Further key information to be obtained from Rod 2 is the dimension of the burst opening. The burst opening size together with the amount of fuel particles smaller than this size provides an estimation of the potential maximum amount of fuel that can escape the rod through the rupture opening. The consequences of fuel dispersal include fuel-coolant interaction, radiological consequences and hydraulic and mechanical consequences [10].

An estimation of the rupture opening size was performed. The rupture is visible on Figure 3.23 corresponding to the visual inspection 180 degree orientation. The burst opening size is very small with a length of about $2.5 \mathrm{~mm}$ and opening of about $0.3 \mathrm{~mm}$.

The visual inspection of Rod 3 shows that the white spots on the black cladding outer diameter oxide as a result of cladding strain and oxide cracking observed for Rod 1 and Rod 2 are not present, confirming that the cladding strain was very small. 


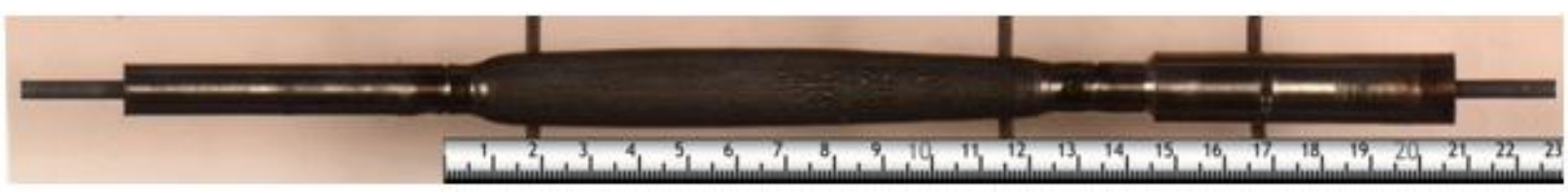

Figure 3.19: Visual inspection Rod 1 (0 orientation)

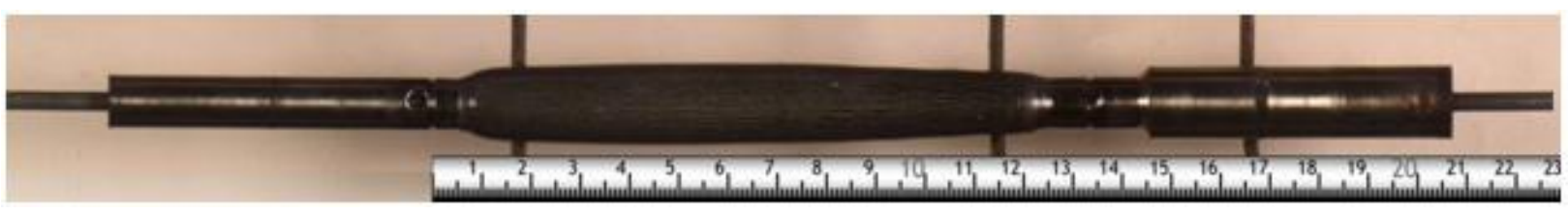

Figure 3.20: Visual inspection Rod 1 (90 orientation)

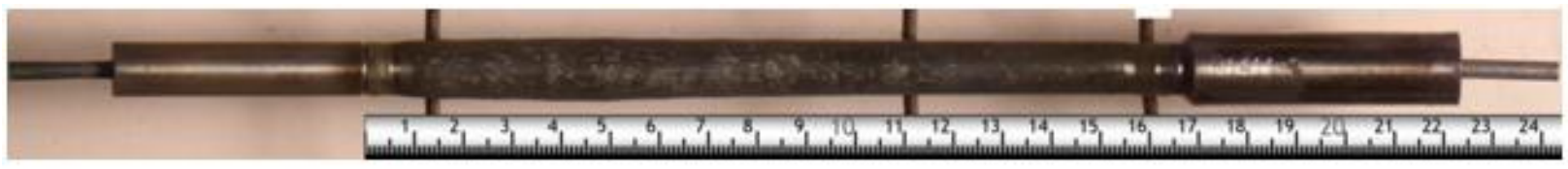

Figure 3.21: Visual inspection Rod 2 (0 orientation)

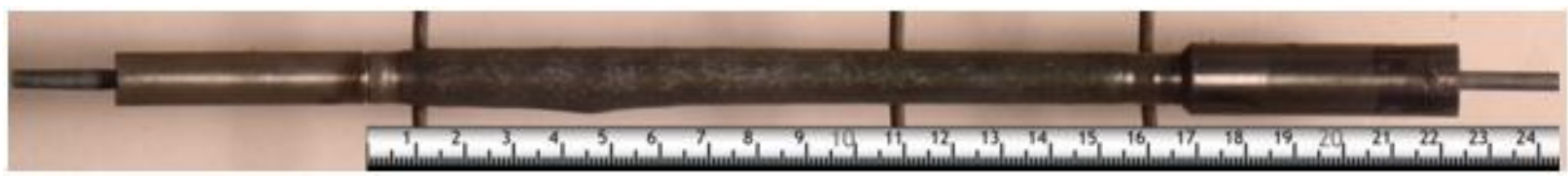

Figure 3.22: Visual inspection Rod 2 (90 orientation)

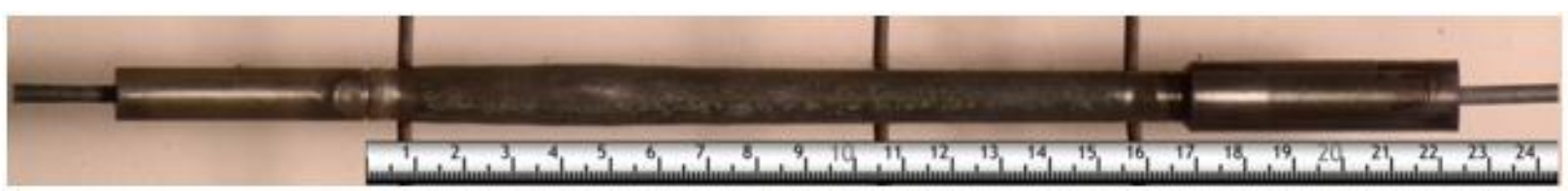

Figure 3.23: Visual inspection Rod 2 (180 orientation)

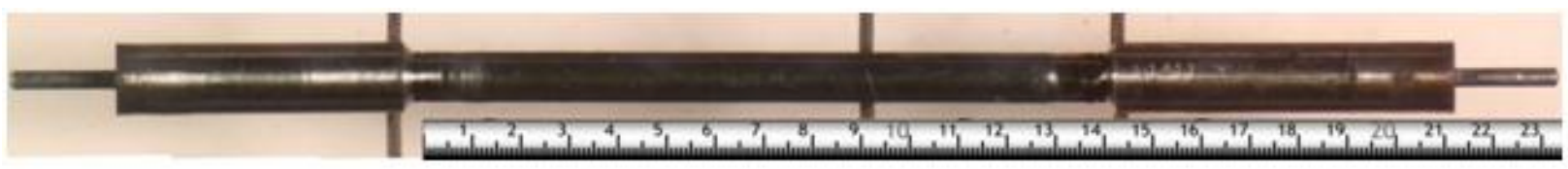

Figure 3.24: Visual inspection Rod 3 (0 orientation)

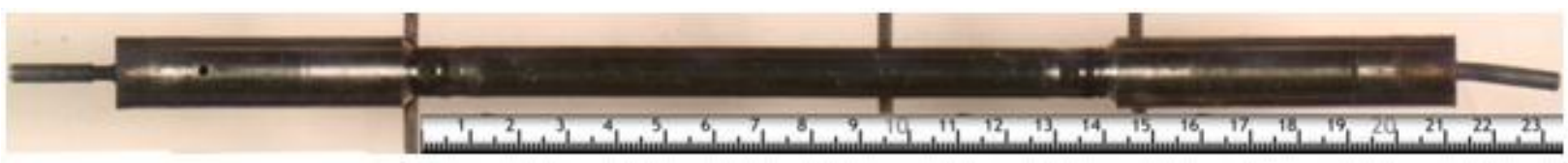

Figure 3.25: Visual inspection Rod 3 (90 orientation) 


\subsubsection{Cladding diameter measurement}

The cladding diameter was measured by contact profilometry inside the hot cells. These measurements were performed for the three Rods prior to and after the experiment execution to obtain the cladding deformation during the transient. The data collected have been used to benchmark the deformation calculated with TRANSURANUS. TRANSURANUS was extensively validated against experimental data and the latest version of the code [11] was extended in order to take into account large creep strains for the simulation of clad ballooning occurring during loss-of-coolant accidents (LOCA).

Figure 3.26, 3.27 and 3.28 show the collected axial dimension data for the three rods and the associated calculation's results given by TRANSURANUS.

The cladding deformation during the transient heating up is influenced by the development of inner rod pressure and cladding temperature. The inner rod pressure is influenced by the initial pressure level, the cladding deformation with a consequential increase of volume inside the rod and the transient fission gas release that corresponds to an increase of inner rod's pressure level during the transient. The fission gas release during the transient is due to fission gas diffusion within the fuel matrix because of the heating up, fuel fragmentation and the release from the high burnup structure. These phenomena are not taken into account by the current and available version of TRANSURANUS.

The benchmark between the cladding deformation measurement and TRANSURANUS for Rod 1 shows that TRANSURANUS predicts a cladding deformation profile that is not consistent with the experimental results of the profilometry analysis.

The inconsistency can be explained analyzing the inner rod pressure and cladding temperature transient together with the temperature profiles at different time steps calculated by TRANSURANUS as shown in Figure 3.29 and Figure 3.30. It should be underlined that for the benchmark calculation the $x$-axis is inverted with respect to Figures 3.16 - 3.25. The rod inner pressure reached its maximum value of 36 bar at about $700 \mathrm{~s}$ and afterwards ballooning started as shown by the reduction of pressure of Figure 3.29. When ballooning occurs, the temperature distribution along the active fuel length is expected to have a middle-bottom peak profile consistent with the cladding deformation calculated by TRANSURANUS of Figure 3.26. However, as the transient goes on the temperature distribution tends to assume a very flat profile as shown by Figure 3.30 at $900 \mathrm{~s}$ and $1500 \mathrm{~s}$. Considering the further increase of temperature, the removal of the geometrical constraints of the cladding that induces fuel cracks and fragmentation and the transient fission gas release (see 3.4.3), the pressure in the transient drops eventually less than shown in Figure 3.29 and this level of pressure together with a more flat temperature profile as shown in Figure 3.30 may induce further deformation at the centre and top of the cladding as measured by the profilometry. It should be also underlined that in TRANSURANUS axial cladding nodes are not strongly coupled, i.e. if one node is ballooning the other nodes more or less are unaffected and therefore the resolution for small segments is reduced. 
The benchmark calculation for Rod 2 shows a good agreement between the measurement data and the TRANSURANUS calculation in the central and top part of the fuel. TRANSURANUS predicts with a good accuracy the burst location even though the cladding deformation at burst is over predicted. This over prediction was also found in [12] and it is dependent upon the very detailed axial nodalization and can be softened with larger axial nodes. It should be underlined that Rod 2 is not so impacted by the transient FGR because the FGR release after fuel rod burst pressurizes directly the capsule environment.

The profilometry for Rod 3 as in Figure 3.28 shows an average cladding diameter after the test of 10.78 $\mathrm{mm}$ and therefore an average cladding deformation of about $0.03 \mathrm{~mm}$ as confirmed by the TRANSURANUS calculation.

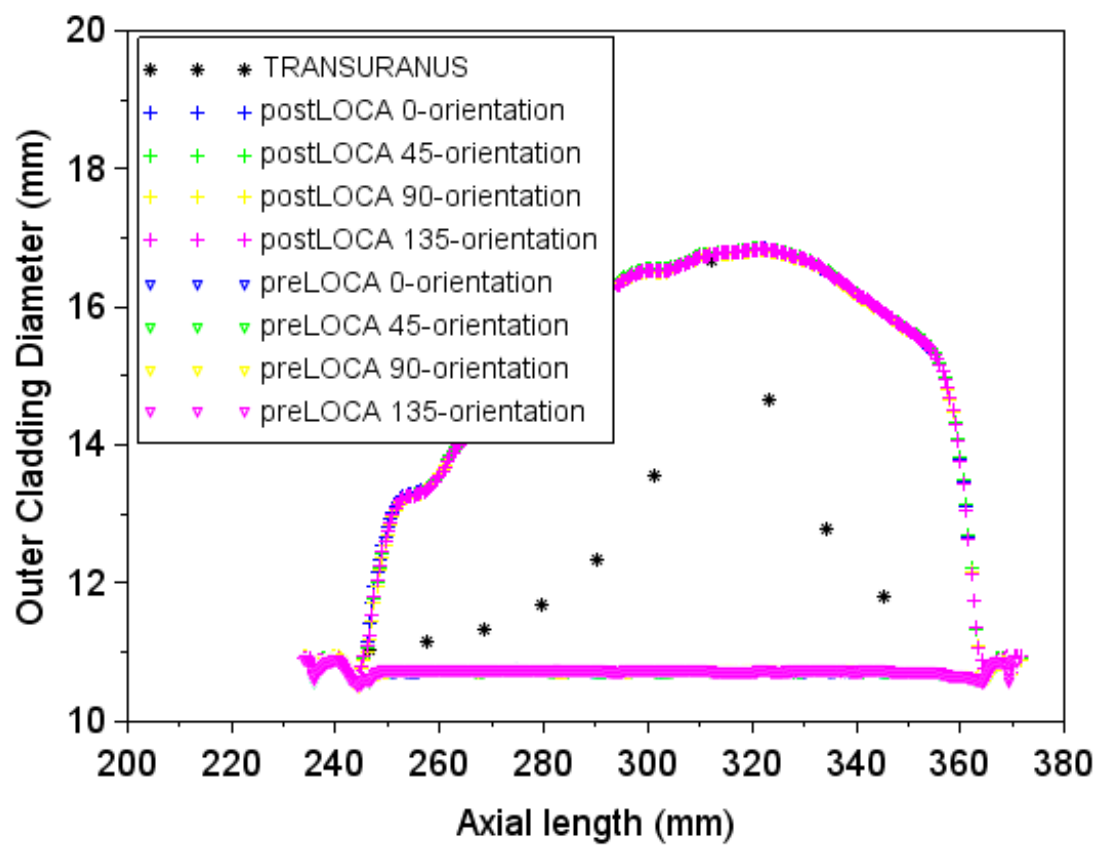

Figure 3.26: Rod 1 Cladding Deformation data vs TRANSURANUS 


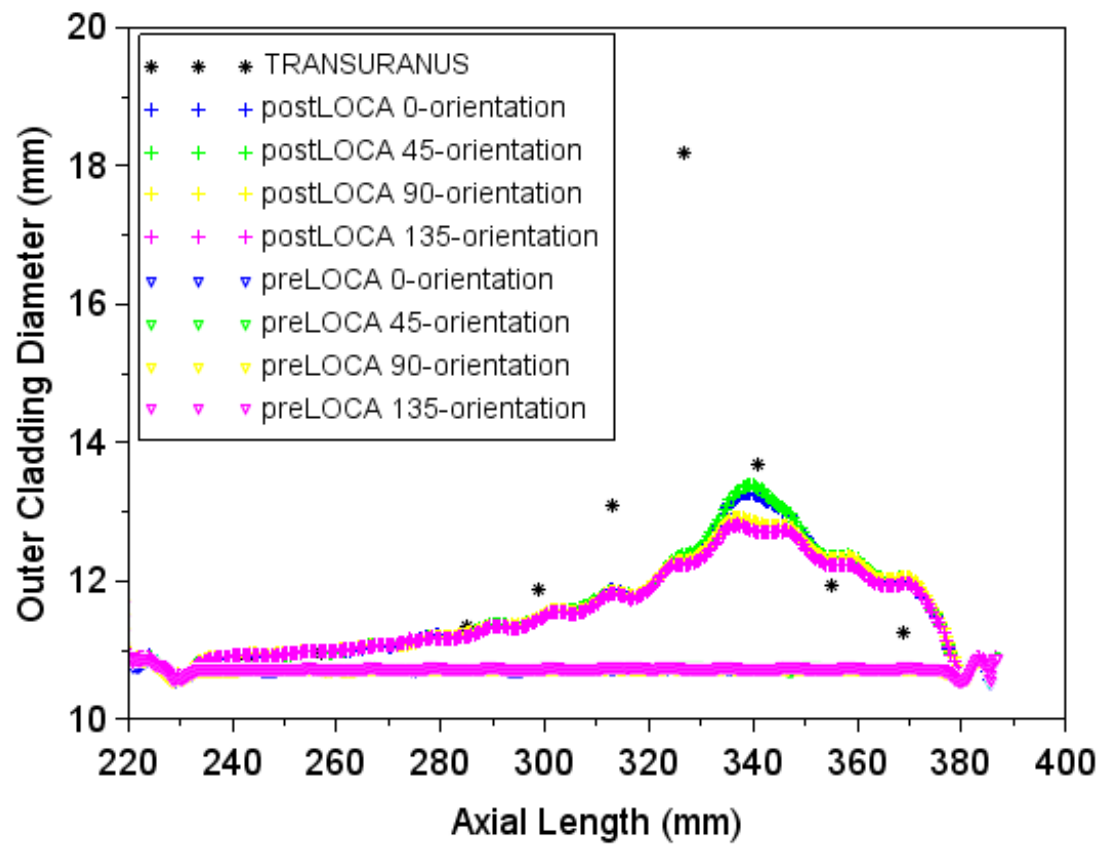

Figure 3.27: Rod 2 Cladding Deformation data vs TRANSURANUS

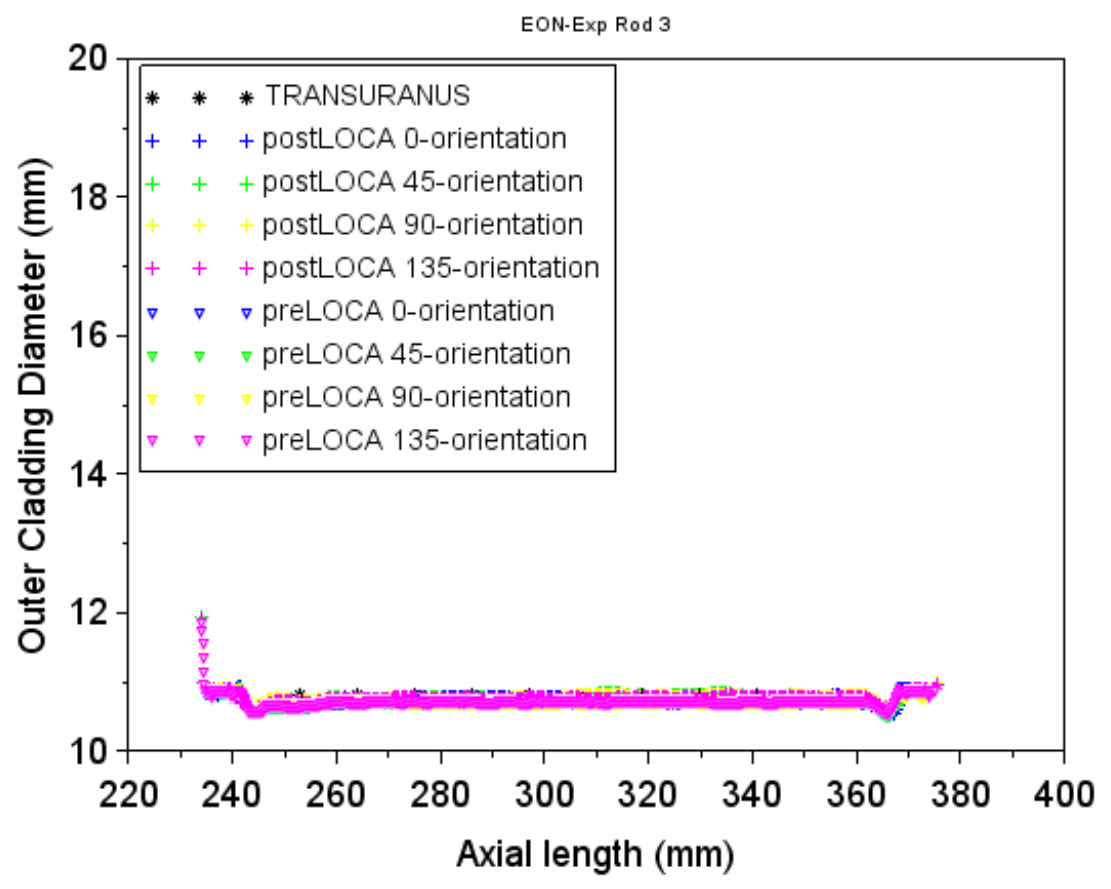

Figure 3.28: Rod 3 Cladding Deformation data vs TRANSURANUS 

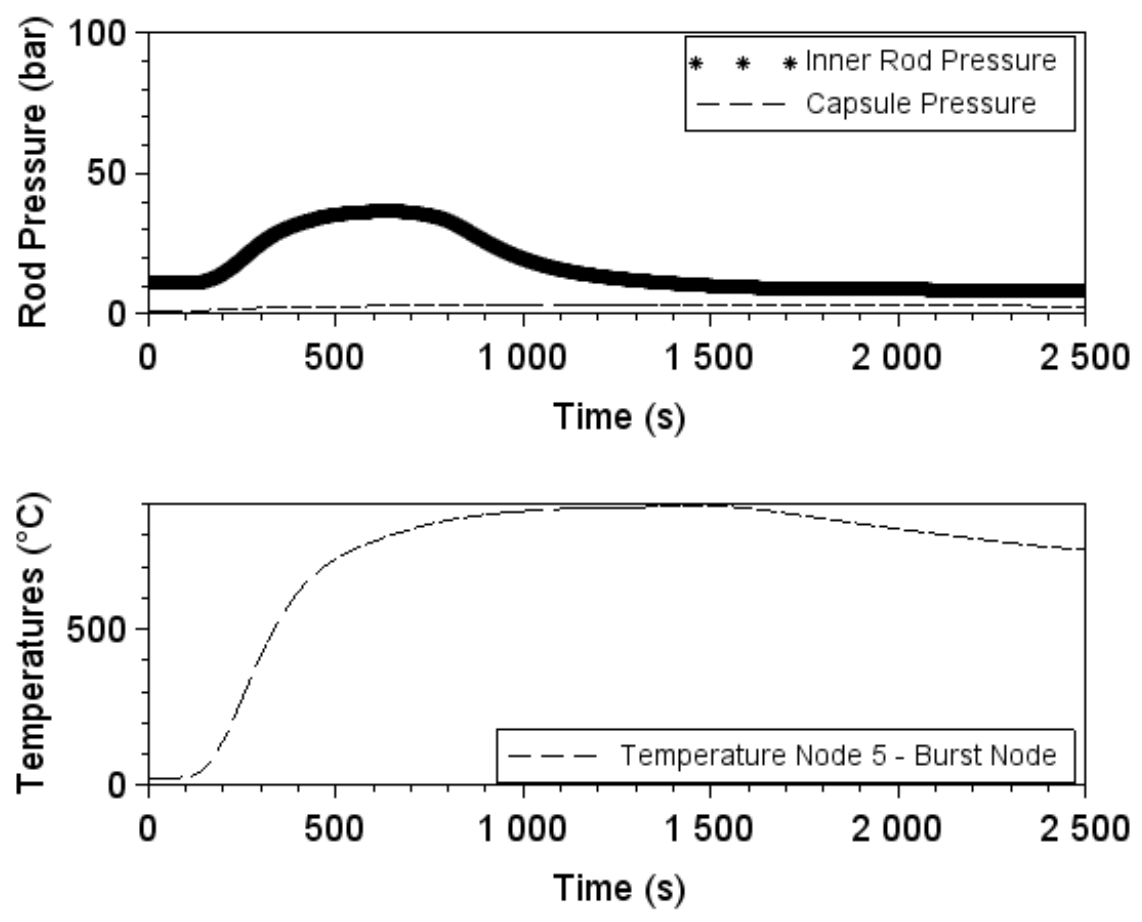

Figure 3.29: Rod 1 inner and outer rod pressure calculated by TRANSURANUS

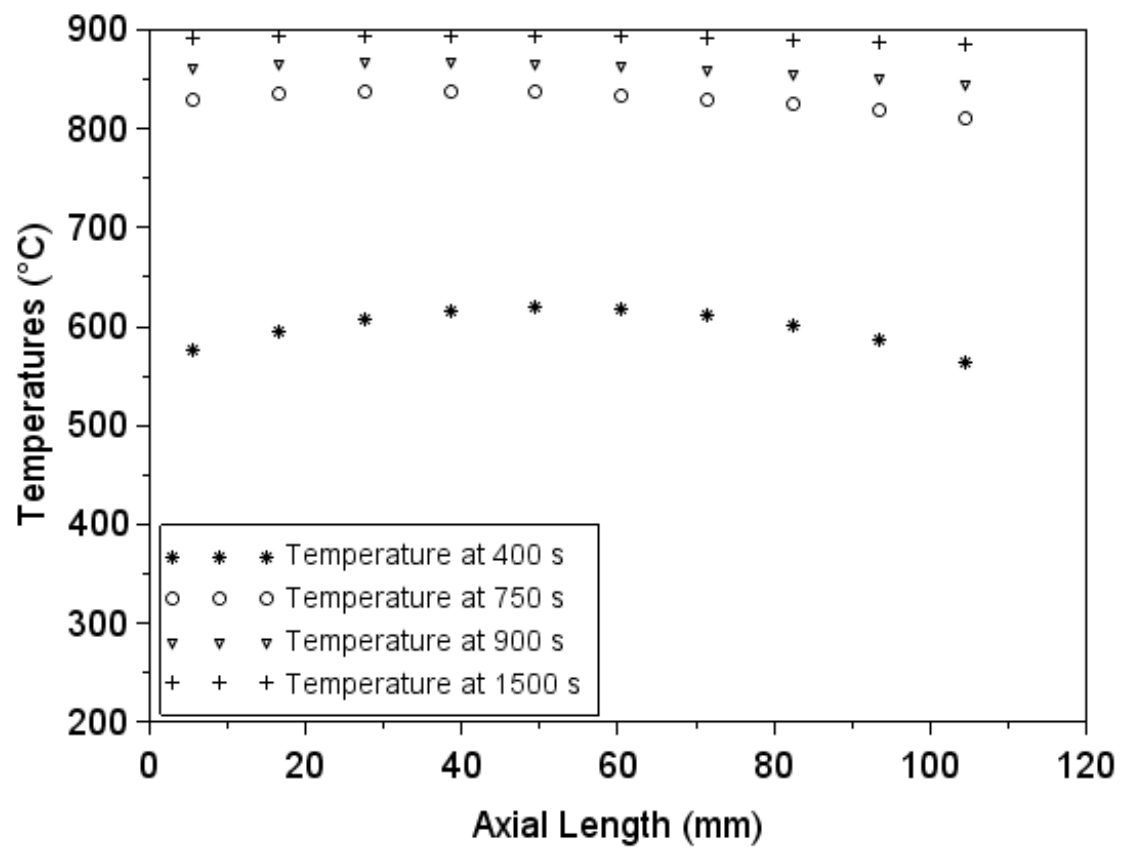

Figure 3.30: Rod 1 temperature profiles during the transient calculated by ATHLET 


\subsection{Destructive Investigation}

The second phase of PIE included different destructive investigations performed at the HRP laboratory and hot cells in Kjeller. A ceramography was performed on the archive pellet not subject to the heating up transient to appraise the status of the fuel prior to the test (3.4.1). At a later stage the rod segments 1 and 3 have been punctured and the gas was collected in independent samples. The puncturing of Rod 2 was not required since the rod burst during the heating up and the pressure transducer piping system allowed the collection of the gas release from the rod. Afterwards the rod segments 1 and 2 have been defueled and the fuel particle size analysis was done to determine the fragment size distribution (3.4.2). The gas collected from the three rods segments have been used to various purposes to determine the integral amount of fission gas released during the transient (3.4.3) and to perform a gamma activity measurement of the three samples (3.4.4).

\subsubsection{Ceramography of archive pellet}

The ceramography of the archive pellet was performed in the HRP laboratory.

Figure 3.31 shows a transversal cross section of an archive fuel pellet that was taken from the main irradiated fuel segment and stored before to perform the test. The ceramography reveals the crack patterns and zones of varied appearances. Cracks are mainly radially oriented with no influence on the radial heat transfer coefficient at different azimuthal locations. The pellet cladding gap seems closed but no relevant radial relocation is observed.

Figure 3.32, Figure 3.33 and Figure 3.34 provide details of the center, intermediate and external rim zone of the pellets. The central radial region shows mainly planar defects visible as dark features within the grain. Details of the rim region show redistribution of the extended defects and high-concentration of micron-sized intergranular closed pores characteristic of the HBS. The thickness of the HBS seems very limited and this information is consistent with the TRANSURANUS irradiation calculation that predicts a thickness of the transient HBS of circa $0.33 \mathrm{~mm}$.

The fuel pellet represents therefore a typical fuel irradiated in a commercial nuclear power plant up to $52 \mathrm{MWd} / \mathrm{kgU}$ and a comparison can be also made with Figure 2.1 that is also a typical LWR fuel at circa $52 \mathrm{MWd} / \mathrm{kgU}$. 


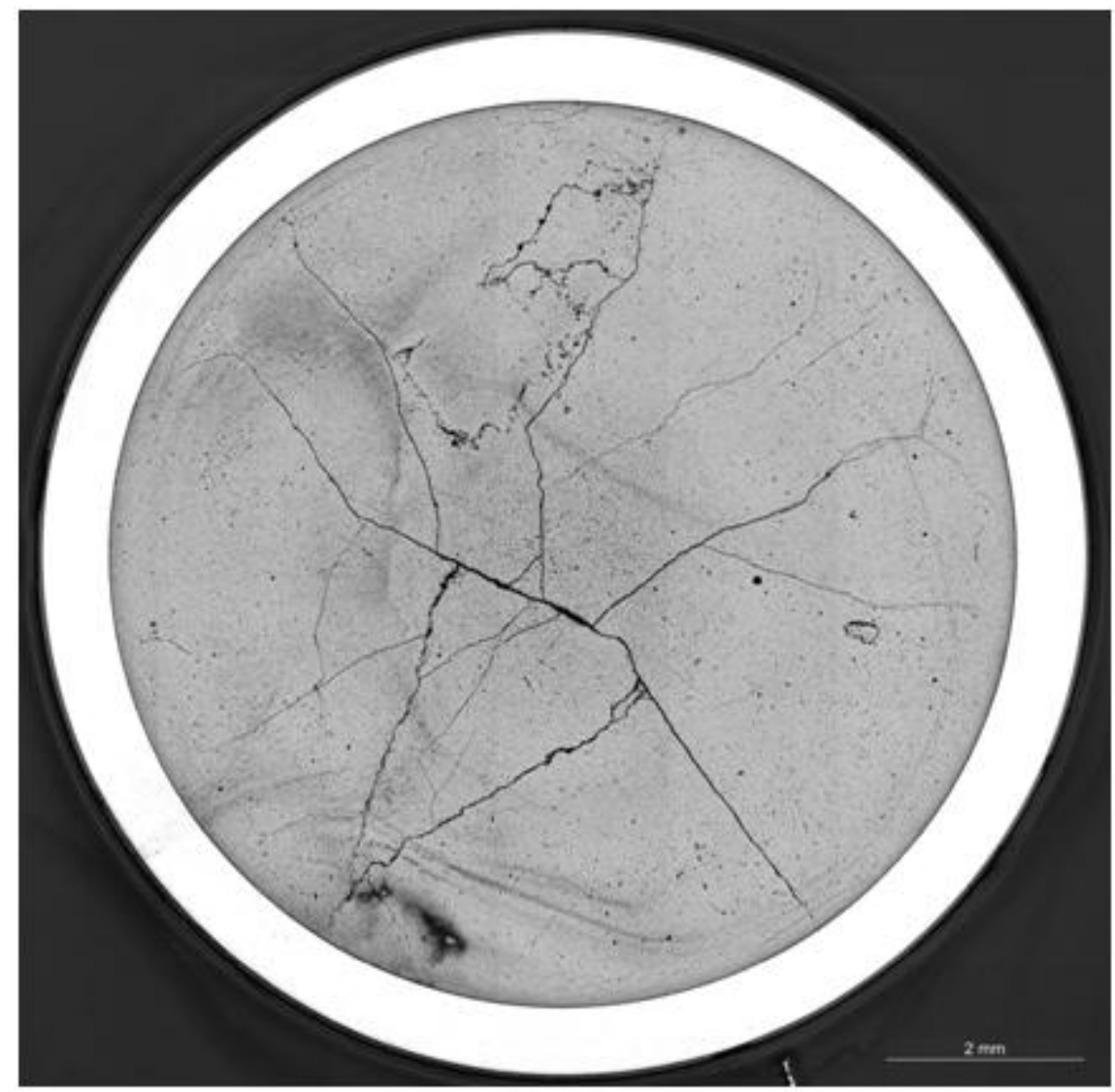

Figure 3.31: Archive pellet ceramography

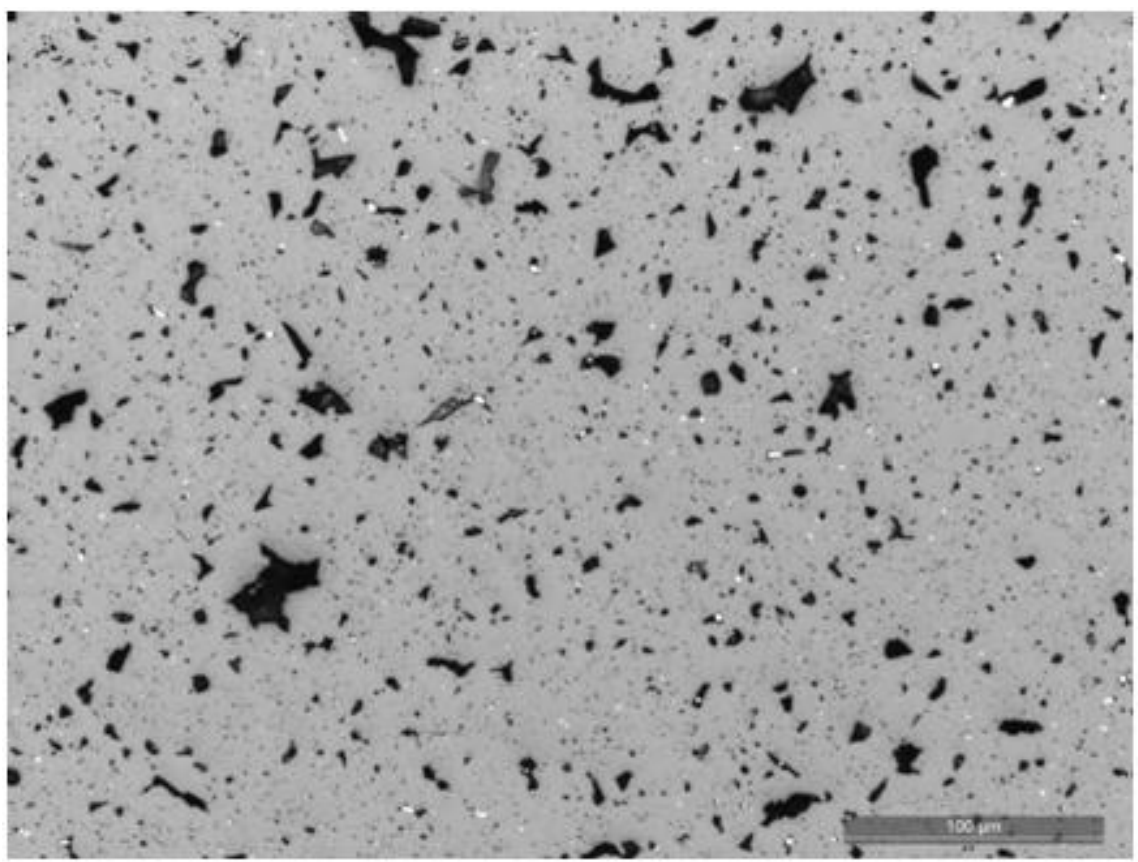

Figure 3.32: Details of ceramography at the center zone of the pellet 


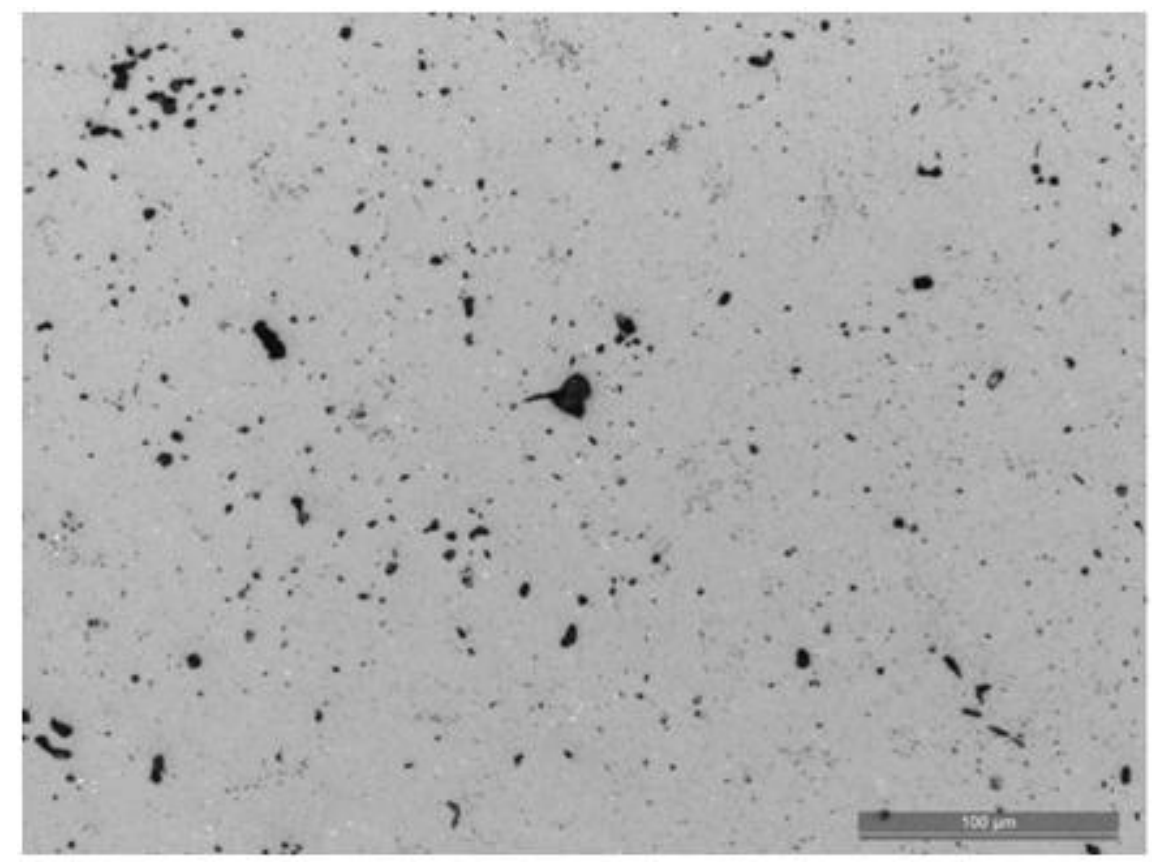

Figure 3.33: Details of ceramography at the intermediate zone of the pellet

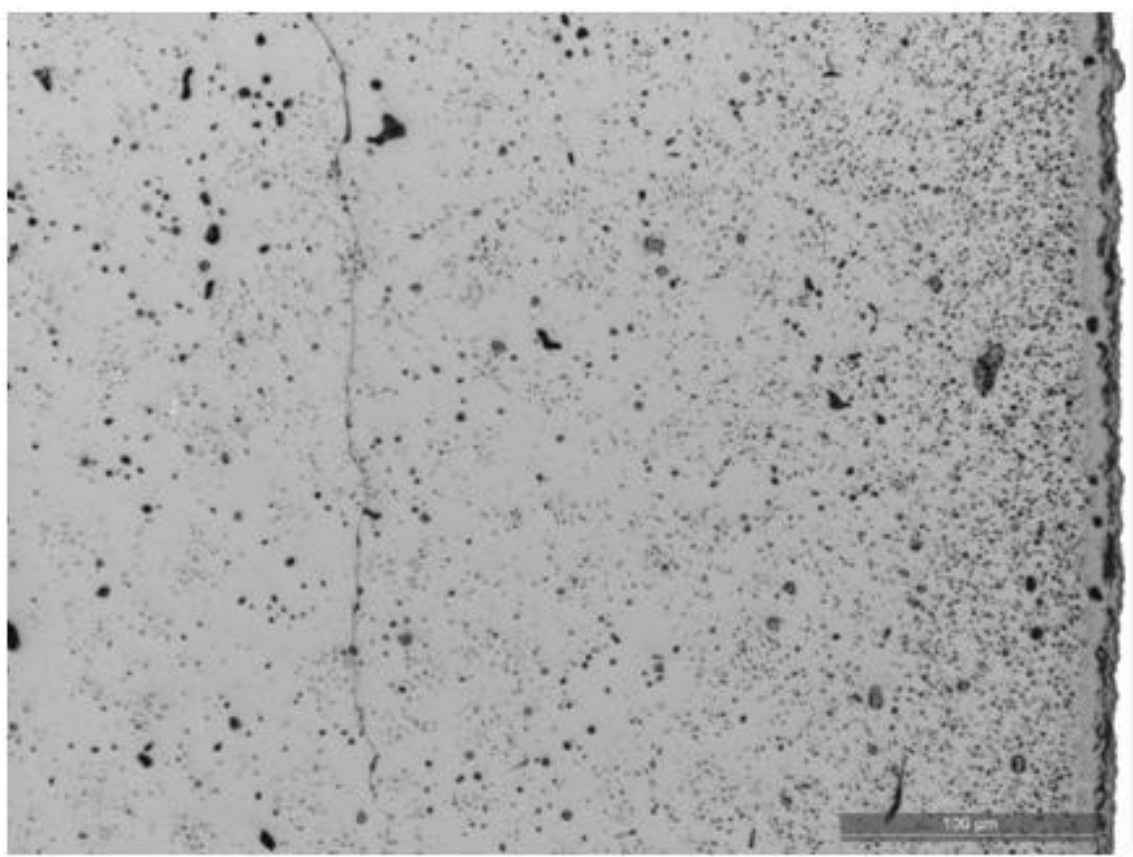

Figure 3.34: Details of ceramography at the outer zone of the pellet 


\subsubsection{Fuel Particles Sifting}

The fragment size distribution of fuel rod segment Rod 1 and Rod 2 was determined experimentally. The main steps in the fuel fragment size determination included cutting of the fuel segment, defueling, and sifting of removed particles in a standard ASTM sifting system. Figure 3.35 a) shows the ASTM sifting system used with grid sizes: $4.0 \mathrm{~mm}, 2.0 \mathrm{~mm}, 1.0 \mathrm{~mm}, 0.50 \mathrm{~mm}, 0.25 \mathrm{~mm}$, and $0.125 \mathrm{~mm}$. Motion during sifting was obtained by a vibrator placed below the systems of the sifts. Figure $3.35 \mathrm{~b}$ ) shows the sifting operation inside the hot cells.

The individual sifts in the system were weighed without and with particles. The collected particles on the individual sifts were documented by photos as shown in Figure 3.36 for Rod 1 and Rod 2. Prior to defueling, the segment was cut just above and below the active fuel stack. Subsequently all rod pieces were defueled mechanically by shaking with a manipulator in a hot-cell. With this technique, all loose fuel particles could be removed. The particles size-distribution by weight of collected particles is given in Table 3.2.

In Rod 2 (burst) and Rod 1 (no burst) less than 3.0\% of the total fuel weight was fragmented into particles of $2 \mathrm{~mm}$ and smaller. Considering also the maximum opening size determined in 3.3.2, the amount of fuel dispersal of from the Rods was negligible.

The fuel sifting presented interesting results in showing smaller fuel fragments for Rod 1 than for Rod 2. The neutron radiography analysis showed fuel fragmentation of 3 pellets of Rod 2 close to the ballooning and burst region and cracks on the pellets nearby and only fragmentation for Rod 1. However, it should be underlined that the resolution of the neutron radiography is about $40 \mu \mathrm{m}$, and seemingly intact pellets seen on neutron radiography can have cracks.

These results can be interpreted based on a physical explanation and/or the experimental procedure. Rod 1 did not experience cladding burst, however the removal of the cladding's geometrical constraint occurred for the entire length of the fuel. Due to the removal of the geometrical constraint it is possible that cracks occurred in the fuel along the entire length of the fuel and they were not detected by the neutron radiography. The ballooning in Rod 2 did not evolve for the entire axial length of the fuel as shown by the cladding diameter measurements. Therefore, the fuel pellets of Rod 1 presented more cracks due to the removal of the geometrical cladding constraints along the entire axial length of the fuel $(11 \mathrm{~cm})$ and this effect compensated the fuel fragmentation of Rod 2 in the burst region given by the removal of the constraint of the inner rod pressure.

It should be underlined that the operation of mechanical defueling and shaking during the particle size analysis may have had perturbed the results.

The fuel fragment size distribution for Rod 1 and Rod 2 is shown in Figure 3.37. 


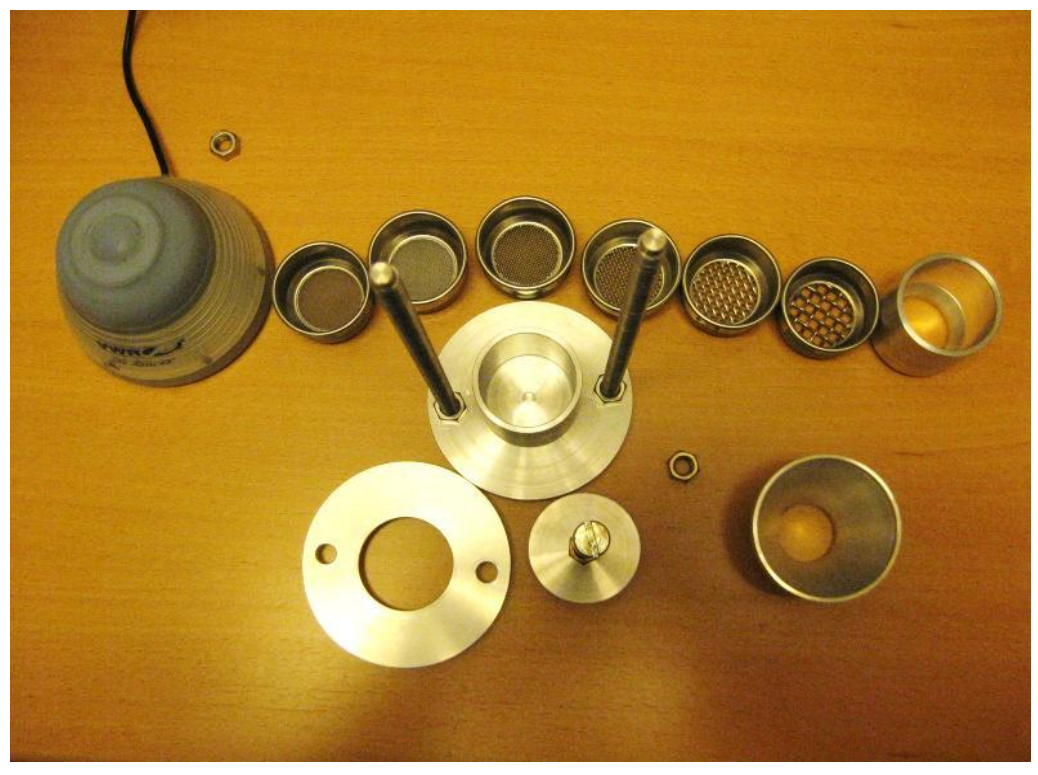

a)

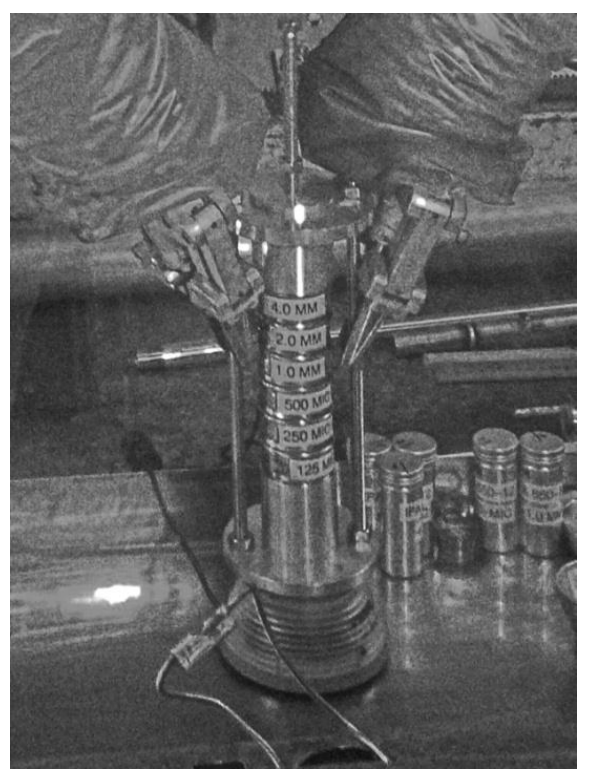

b)

Figure 3.35: Sifting operation

a) sifts of different sizes and shaking system; b) sifting operation in the hot cells

\begin{tabular}{|c|c|c|}
\hline Particle Size & Rod 1 & Rod 2 \\
\hline$\geq 4 \mathrm{~mm}$ & $68.107 \mathrm{~g}$ & $71.051 \mathrm{~g}$ \\
\hline $4-2 \mathrm{~mm}$ & $2.519 \mathrm{~g}$ & $0.97 \mathrm{~g}$ \\
\hline $2-1 \mathrm{~mm}$ & $0.688 \mathrm{~g}$ & $0.356 \mathrm{~g}$ \\
\hline $1-0.5 \mathrm{~mm}$ & $0.24 \mathrm{~g}$ & $0.143 \mathrm{~g}$ \\
\hline $0.5-0.25 \mathrm{~mm}$ & $0.192 \mathrm{~g}$ & $0.082 \mathrm{~g}$ \\
\hline $0.25-0.125 \mathrm{~mm}$ & $0.336 \mathrm{~g}$ & $0.089 \mathrm{~g}$ \\
\hline$\leq 0.125 \mathrm{~mm}$ & $0.615 \mathrm{~g}$ & $0.141 \mathrm{~g}$ \\
\hline
\end{tabular}

Table 3.2: Particle sizes analysis results

The total mass of the two rods is different due to the different segment lengths 


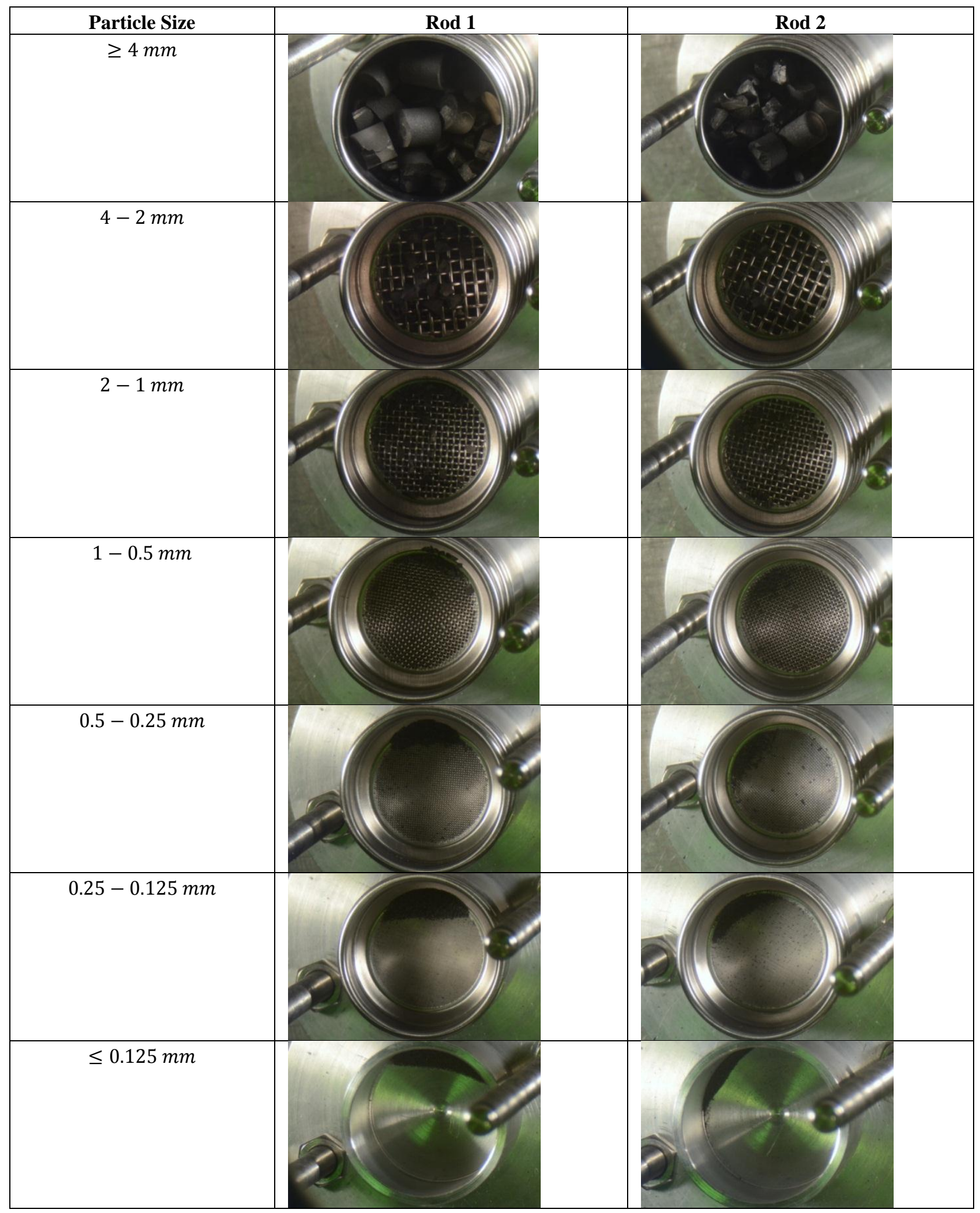

Figure 3.36: Particle sizes analysis results 


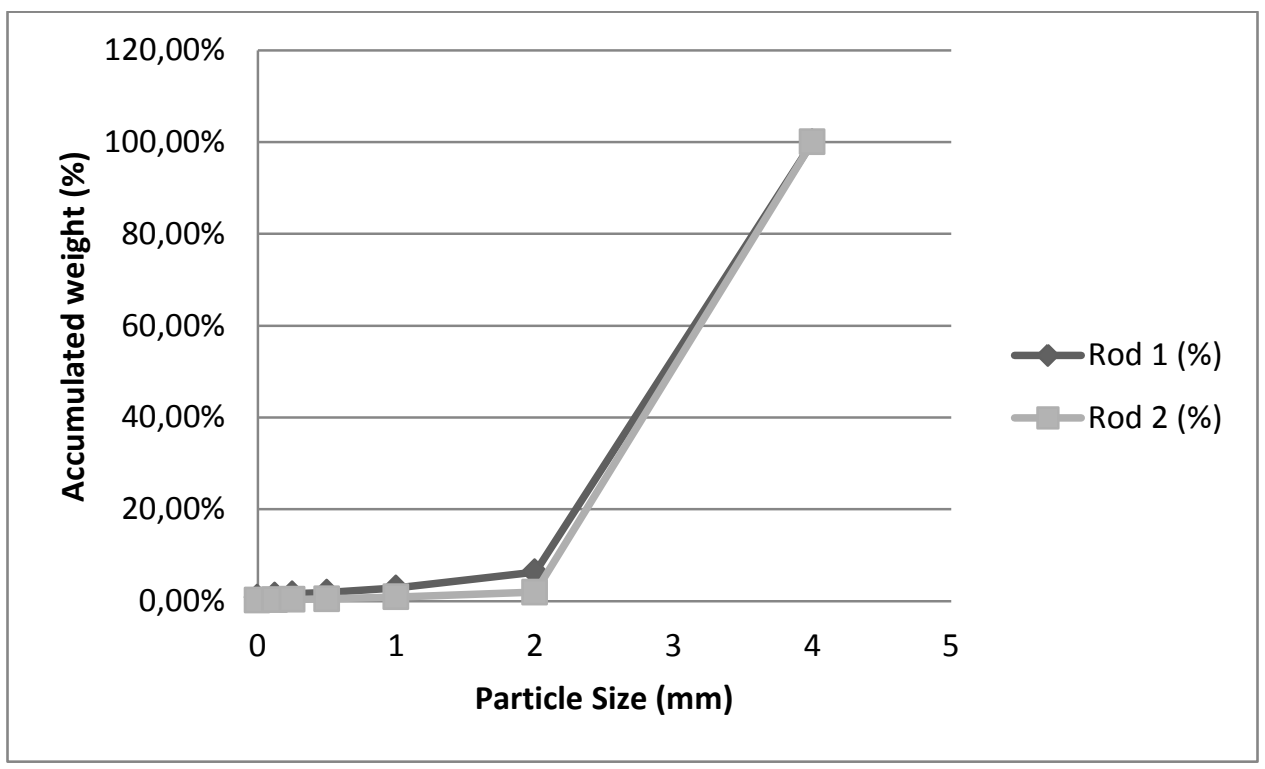

Figure 3.37: Rod 1 and Rod 2 fragment size cumulative distribution

\subsubsection{Transient Fission Gas Release}

The experiment was designed to collect and analyze the fission gas released during the transient. The amount of fission gas release during a DBA is a topic of particular interests for fuel vendors, utilities and regulators [13]. The separate effect test with the capsule surrounding the fuel rod had the advantage to collect the fission gas released from the fuel also of the burst rod. In-pile tests currently do not allow the collection of this gas that is released in the fuel channel environment and the information is consequently lost. The gas analysis includes an estimation of the fission gas released from the three rods during the transient based on measured data and TRANSURANUS prediction.

The gas collected from the three Rods was the sum of the fill-in gas (Helium) and the fission gas released from the fuel during the transient. The collection was performed with two different procedures: 1) the gas from Rod 2 was collected from the capsule piping system because the Rod experienced burst: consequently the gas collected contains the gas release from the fuel rod but also the gas (Helium) of the capsule/pressure transducer system; 2 ) the gas from Rod 1 and Rod 3 was collected by puncturing of the rods.

The estimation of fission gas release from Rod 2 was performed based on the difference prior and after the test of the gas contained in the capsule/pressure transducer system and the gas contained in the fuel rod. The number of moles pre-test $n_{2}^{\text {pre-test }}$ is therefore the sum of the moles contained in the two systems of above. The estimation was performed using the ideal gas law. 


$$
n_{2}^{\text {pre-test }}=\frac{p_{2, \text { capsule }}^{\text {pre-test }} \cdot V_{2, \text { capsule }}^{\text {pre-test }}}{R \cdot T_{2}^{\text {pre-test }}}+\frac{p_{2}^{\text {fill-in }} \cdot V_{2}^{U F V}}{R \cdot T_{2}^{R T}}
$$

The values of pressure $p_{2, \text { capsule }}^{\text {pre-test }}$ and temperature $T_{2}^{\text {pre-test }}$ can be read from the pressure transducer and thermocouples before the test execution and $R$ is the universal gas constant. The volume $V_{2, c a p s u l e}^{\text {pre-test }}$ included the capsule volume, the gas line volume and the pressure transducer system volume. The value of the fill-in pressure $p_{2}^{\text {fill-in }}$ and the upper free volume $V_{2}^{U F V}$ can be read from Table 2.7.

To calculate the number of moles post-test $n_{2}^{\text {post-test }}$ the following formula can be used:

$$
n_{2}^{\text {post-test }}=\frac{p_{2, \text { capsule }}^{\text {post-test }} \cdot V_{2, \text { capsule }}^{\text {post-test }}}{R \cdot T_{2}^{\text {post-test }}}(\text { Formula 4.2) }
$$

Where:

$$
V_{2, \text { capsule }}^{\text {post-test }}=V_{2, \text { capsule }}^{\text {pre-test }}+V_{2}^{U F V}(\text { Formula 4.3) }
$$

In fact, when the rod burst, the fuel rod environment was connected with the capsule/pressure transducer system environment.

The values of pressure $p_{2 \text {,capsule }}^{\text {post-test }}$ and temperature $T_{2}^{\text {post-test }}$ can be read from the instrumentation devices. It should be underlined that the evaluation pre- and post-test was performed in cold condition. The integral value of fission gas release for $\operatorname{Rod} 2 \Delta n_{2}$ can be calculated as:

$$
\Delta n_{2}=n_{2}^{\text {post-test }}-n_{2}^{\text {pre-test }} \quad \text { (Formula 4.4) }
$$

The estimation of fission gas release for Rod 1 and Rod 3 was performed by puncturing the rod. The fuel rod was punctured in the plenum region in the lower end. Expansion pressure became stable after puncturing and consequently no problems with degraded gas communication were experienced under puncturing of the rod. For Rod 1 , the number of moles $n_{1}^{\text {pre-test }}$ can be estimated knowing the dimension of the upper free volume $V_{1}^{U F V}$ and the fill-in gas pressure $p_{1}^{\text {fill-in }}$ performed at room temperature $T_{1}^{R T}$.

$$
n_{1}^{\text {pre-test }}=\frac{p_{1}^{\text {fill-in }} \cdot V_{1}^{U F V}}{R \cdot T_{1}^{R T}} \quad \text { (Formula 4.5) }
$$

The fuel was punctured with the pressure transducer attached; therefore the amount of pressure post test $p_{1}^{\text {post-test }}$ was determined. In addition, the value of the rod free volume after the test $V_{1}^{\text {post-test }}$ that included the initial upper free volume and the volume generated by the cladding ballooning was calculated. The operation was performed at room temperature.

$$
n_{1}^{\text {post-test }}=\frac{p_{1}^{\text {post-test }} \cdot V_{1}^{\text {post-test }}}{R \cdot T_{1}^{R T}}(\text { Formula 4.6) }
$$

The integral value of fission gas release for Rod $1 \Delta n_{2}$ can be calculated as: 


$$
\Delta n_{1}=n_{1}^{\text {post-test }}-n_{1}^{\text {pre-test }} \quad \text { (Formula 4.7) }
$$

and a similar procedure was followed for Rod 3. Table 3.3 collects the parameters described above.

\begin{tabular}{|c|c|c|c|c|}
\hline Parameter & $\operatorname{Rod} 1$ & Rod 2 & Rod 3 & Units \\
\hline$V_{2, \text { capsule }}^{\text {pre-test }}$ & na & 86.4 & na & $\mathrm{cc}$ \\
\hline$p_{2, \text { capsule }}^{\text {pre-test }}$ & na & 0.99 & na & bar \\
\hline$T_{2}^{\text {pre-test }}$ & na & 22 & na & ${ }^{\circ} \mathrm{C}$ \\
\hline$p_{2, \text { capsule }}^{\text {post-test }}$ & na & 2.43 & na & bar \\
\hline$T_{2}^{\text {post-test }}$ & na & 22 & na & ${ }^{\circ} \mathrm{C}$ \\
\hline$V^{U F V}$ & 1 & 5 & 1 & $\mathrm{cc}$ \\
\hline$p^{\text {fill-in }}$ & 10 & 14 & 0.7 & bar at RT \\
\hline$p^{\text {post-test }}$ & 1.27 & na & 1.28 & bar at RT \\
\hline$V^{\text {post-test }}$ & 12.59 & na & 5.57 & $\mathrm{cc}$ \\
\hline
\end{tabular}

Table 3.3: Parameters for fission gas release calculation

Cc: cubic centimeter; na: not applicable: the measurement for Rod 1 and 2 was performed by rod puncturing and information about the capsule were not relevant

To determine the percentage amount of fission gas release required the estimation of the fission gas generated in the fuel during irradiation. The TRANSURANUS irradiation calculation performed in 2.3.1 was used for this purpose. The fuel segments have different length: $11 \mathrm{~cm}$ for Rod 1 and Rod 3 and 14 $\mathrm{cm}$ for Rod 2. Consequently, the integral amount of fission gas is different. The fission gas generated in the segments is 110.37 cc for Rod 1 (and Rod 3) and 140.47 cc for Rod 2. During irradiation, fission gas generated in the fuel is distributed in the grain and grain boundaries [14], in the high burnup structure [15] and partly is released in the fuel/cladding gap environment [16]. TRANSURANUS predicts a distribution of fission gases of $96.32 \%$ in the grain, $1.40 \%$ in the grain boundaries, $1.93 \%$ in the high burnup structure and $0.35 \%$ of fission gas release which is lost when opening the original rod.

Table 3.4 summarizes the fission gas production in the pellets expressed in moles of $\mathrm{Xe}$ and $\mathrm{Kr}$ and the calculated values of moles released from the three rods based on the formula of above.

\begin{tabular}{|l|c|c|c|}
\hline \multicolumn{1}{|c|}{ Parameter } & Rod 1 & Rod 2 & Rod 3 \\
\hline $\begin{array}{l}\text { Transient fission gas } \\
\text { release experimentally } \\
\text { obtained }(\mu \mathrm{mol})\end{array}$ & 293.76 & 457.97 & 170.33 \\
\hline $\begin{array}{l}\text { Fission Gas Generated } \\
\text { calculated by } \\
\text { TRANSURANUS }(\mu \mathrm{mol})\end{array}$ & 4923.87 & 6266.77 & 4923.87 \\
\hline $\begin{array}{l}\text { Transient Fractional FGR } \\
(\%)\end{array}$ & $5.97 \%$ & $7.31 \%$ & $3.46 \%$ \\
\hline
\end{tabular}

Table 3.4: Estimation of fission gas release from the three rods

The highest value of fission gas release is found to be occurring to Rod 2 that experienced ballooning and burst, than Rod 1 that experienced only ballooning and third Rod 3 that did not experienced cladding deformation. 
The three fuel rods experienced a similar heating up and consequently the fission gas release measured for Rod 3 is common to the three rods and it is due to the diffusion of fission gas during the heating up and cracks that can occur also when the constraints are retained. The difference between the fission gas release of Rod 1 and Rod 2 and this common value from Rod 3 is an indication of how the different transient boundary conditions influenced the fission gas released during the transient.

Based on these results, an evaluation can be made to appraise the effect of removal of the constraint on the fission gas release. Since Rod 1 had a much extended ballooning along the entire length of the fuel, the difference between the fission gas release of Rod 1 and Rod 3 is the measure of the fission gas release due to fragmentation for the removal of the cladding constraint (2.51\%). Rod 2 experienced a removal of the constraint similar to Rod 1 for about $55 \%$ of its length (see Figure 3.27). Consequently, an estimation of the fission gas release given by the burst effect can be calculated considering a superposition of effects and dividing Rod 2 in a zone comparable to Rod 1 (55\% of the length balloon region) and second zone comparable to Rod 1 (45\% cladding retention region).

$$
\begin{aligned}
& F G R_{\text {Rod } 2}=F G R_{\text {burst }}-55 \% \cdot F G R_{\text {Rod } 1}-45 \% \cdot F G R_{\text {Rod } 3} \quad \text { (Formula 4.8) } \\
& F G R_{\text {burst }}=F G R_{\text {Rod } 2}-55 \% \cdot F G R_{\text {Rod } 1}-45 \% \cdot F G R_{\text {Rod } 3} \approx 2.5 \% \quad \text { (Formula 4.9) }
\end{aligned}
$$

It is interesting to observe that the removal of each constraint seems to add about $2.5 \%$ fission gas release.

Some considerations are needed to assess the uncertainty of this evaluation, which includes the accuracy of the measurements and correlations used:

- Thermocouples: In accordance with the supplier certificate, the uncertainty of the temperature measurements made with mineral insulated thermocouples (TC) is approximately $\pm 0.2 \%$ of the temperature difference between the hot and cold junctions. As the cold junction was at room temperature, the above statement implies an uncertainty of $\pm 1.8^{\circ} \mathrm{C}$ at a temperature of up to $900^{\circ} \mathrm{C}$, as was the case for the test in question.

- Pressure transducer: For the pressure measurement concerns, the supplier specification states an accuracy of $\pm 0.01 \mathrm{MPa}$, which is applicable to the tests in question because the sensor was placed in ideal conditions, i.e., at room temperature and in instrumentation laboratory free from vibrations and electronic noise.

- Burnup: The differences in the axial burnup are considered negligible, and the absolute burnup calculations are linked to the power histories derived from the core power tracking, which generally has an accuracy of $5 \%$ on a local basis.

- Puncturing: uncertainty of the free volume measurement in the puncturing technique is estimated to be between $5 \%$ and $10 \%$ and considering the propagation of the uncertainty in the calculation.

Considering the accuracy of the measurements as described above, the use of the ideal gas law and the propagation of the uncertainty in the calculation, the uncertainty on the transient fractional FGR is estimated to be approximately $30 \%$. 


\subsubsection{Gamma-Ray Spectroscopy}

Gamma spectroscopy is a well-established and widely used technique for characterizing nuclear fuel, where the analysis of isotope-specific gamma-rays allows investigation of the fuel properties to which the emitting isotopes correspond. Specifically, the content and distribution of the selected isotopes within the fuel is determined and correlated to the respective fuel property [17]. The gamma-ray spectroscopy has several applications in the nuclear technology and Table 3.5 shows the common fuel properties investigated using gamma spectroscopy, along with the corresponding radioactive isotope, its half time and energies of emitted gamma rays. Recently, the use of gamma spectroscopy has found a novel application to enhance the knowledge of the mechanisms and timescales of the FGR phenomenon. Determining FGR, which over short periods of irradiation, is of particular interest in research reactor settings such as at the HBWR, where experiments often involve short irradiation cycles where fuel is subjected to intentional transients during which the fuel behavior is investigated [18].

The fuel segment used in this experiment was irradiated in the 1990s and consequently it is still possible to detect ${ }^{85} \mathrm{Kr}$ traces. The gamma-ray spectroscopy was performed from the three samples containing the collected gasses from the fuel rods and/or capsule. The samples have similar volumes and consequently the ${ }^{85} \mathrm{Kr}$ activity should reflect the amount of isotope in the samples.

\begin{tabular}{|c|c|c|c|}
\hline Fuel Property & Isotope & Half-Life & $\begin{array}{c}\text { Gamma-ray energy } \\
{[\mathbf{k e V}]}\end{array}$ \\
\hline Power Distribution & ${ }^{140} \mathrm{Ba} /{ }^{140} \mathrm{La}$ & 12.75 days & 1596 \\
\hline Burnup & ${ }^{137} \mathrm{Cs}$ & 30.08 years & 662 \\
\hline Integrated Fission Gas Release & ${ }^{85} \mathrm{Kr}$ & 10.75 years & 514 \\
\hline Irradiation history & ${ }^{137} \mathrm{Cs},{ }^{134} \mathrm{Cs}$ & $30.08,2.07$ years & $662,605,796$ \\
\hline Initial Composition $\left(\mathrm{UO}_{2}\right.$ vs. MOX) & ${ }^{134} \mathrm{Cs},{ }^{154} \mathrm{Eu}$ & $2.07,1.6$ years & $605,796,1275$ \\
\hline
\end{tabular}

Table 3.5: Application of gamma-ray spectroscopy and isotopes' properties

Table from Reference [17]

Figure 3.38, 3.39 and 3.40 shows the spectrum from Rod 1, Rod 2 and Rod 3. The peak representing the ${ }^{85} \mathrm{Kr}$ activity of $514 \mathrm{keV}$ is the channel 616-622. All other peaks are related to the background noise in the laboratory. The spectrum shows the biggest number of counts for Rod 2 ( $20.3 \mathrm{cps}$ ) and smaller value for Rod 1 (13.8 cps) and Rod 3 (11.2 cps). Since the comparison is on absolute values, it should be considered that Rod 2 has different length $(14 \mathrm{~cm})$ than Rod 1 and Rod $3(11 \mathrm{~cm})$. A very simple renormalization of the number of counts for Rod 2 to compare the results with the other two Rods bring to a value of $15.95 \mathrm{cps}$.

This analysis seems to confirm the results of the fission gas release of 3.4 .3 showing that the release from Rod 2 is about 30\% bigger than the release from Rod 1 showing the impact of fuel burst in increasing the fission gas release. Rod 3 was also confirmed to have experienced the smallest release. 
It should be underlined that the ${ }^{85} \mathrm{Kr}$ is only a small part of the total amount of Krypton produced in the fuel rod during reactor irradiation. The relative distribution of the different Krypton isotopes is shown in Table 3.6

\begin{tabular}{|c|c|}
\hline Krypton Isotope & Percentage Amount \\
\hline${ }^{82} \mathrm{Kr}[\%$ of total $\mathrm{Kr}]$ & 0.25 \\
\hline${ }^{83} \mathrm{Kr}[\%$ of total $\mathrm{Kr}]$ & 9.64 \\
\hline${ }^{84} \mathrm{Kr}[\%$ of total $\mathrm{Kr}]$ & 35.61 \\
\hline${ }^{85} \mathrm{Kr}[\%$ of total $\mathrm{Kr}]$ & 1.28 \\
\hline${ }^{86} \mathrm{Kr}[\%$ of total $\mathrm{Kr}]$ & 53.22 \\
\hline
\end{tabular}

Table 3.6: Krypton isotopes production distribution

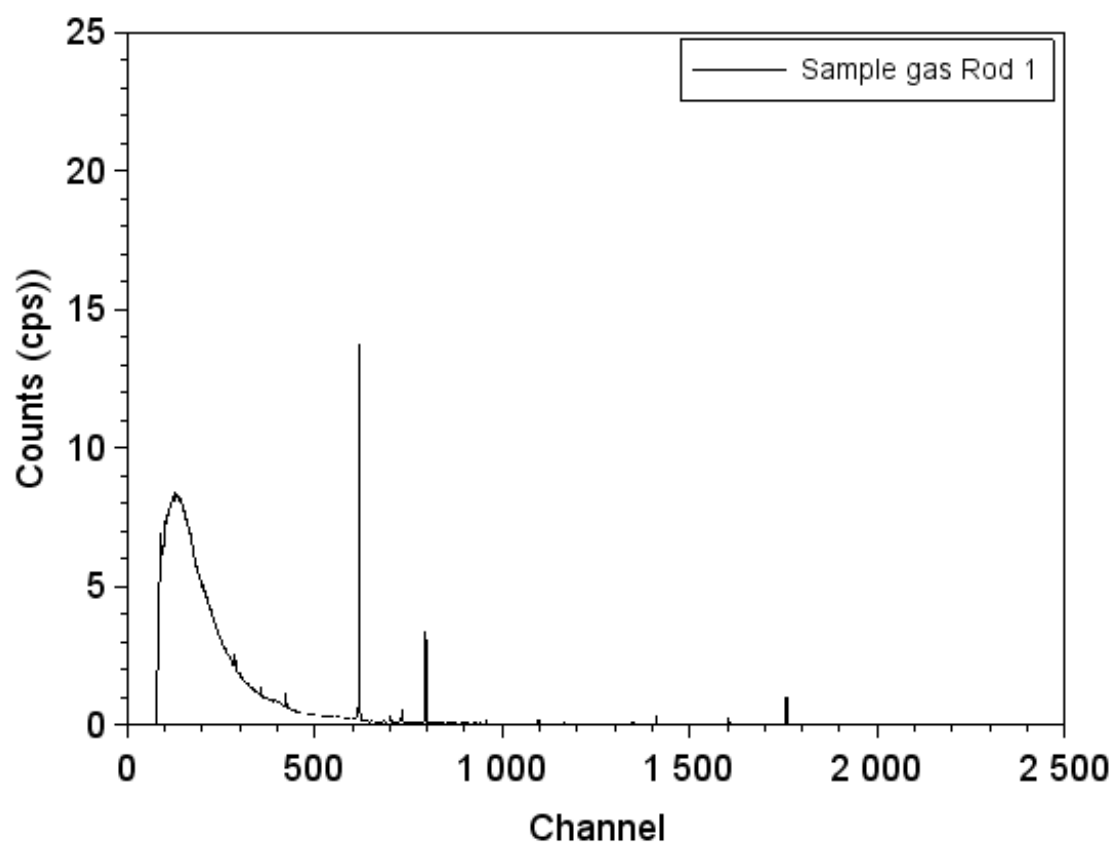

Figure 3.38: Gamma spectrum results for Rod 1 


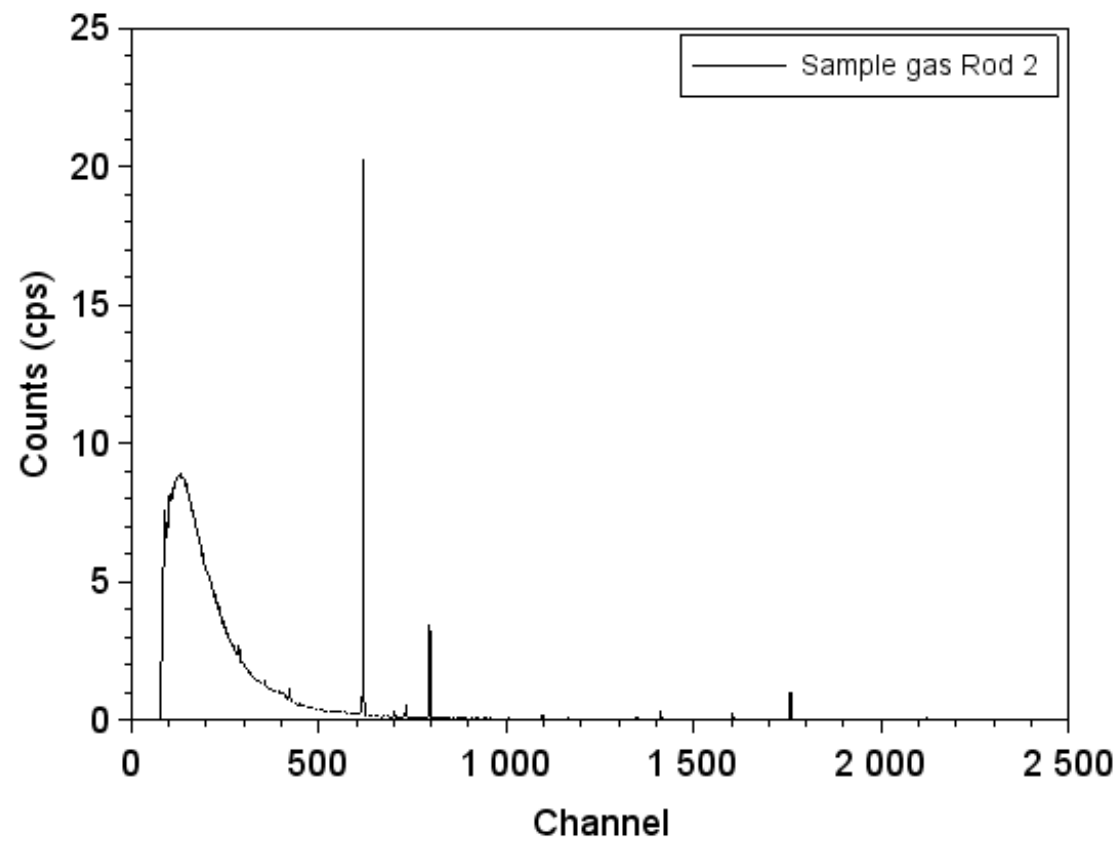

Figure 3.39: Gamma spectrum results for Rod 2

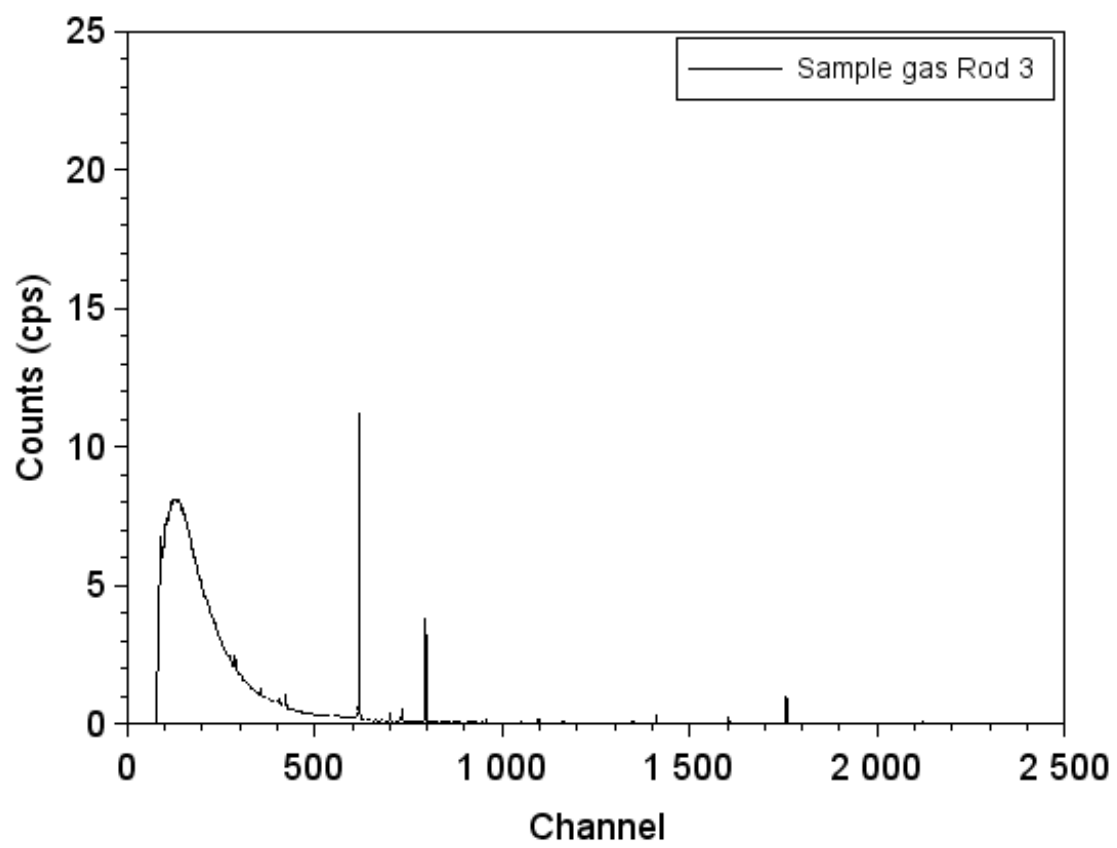

Figure 3.40: Gamma spectrum results for Rod 3 


\section{Bibliography}

[1] P. Auerkari, "Mechanical and physical properties of engineering alumina ceramics", VTT Manufacturing Technology, 1996

[2] N. E. Todreas, M. S. Kazimi, "Nuclear System Volume 1", Table 8.11, Taylor \& Francis Group, 2012

[3] G. Lerchl, H. Austregesilo, P. Schöffel, D. von der Cron, F. Weyermann, "ATHLET Mod 3.0 Cycle A User's Manual“, GRS 2012

[4] "TRANSURANUS handbook", European Commission, JRC Institute for Transuranium Elements 2011

[5] C. Vitanza, "Overview of the OECD-Halden Reactor Project", Nuclear Engineering and Design, Volume 207, Issue 2, July 2001, Pages 207-221

[6] K. Weman, "TIG Welding", Welding Processes Handbook, 2012, Pages 63-69

[7] "Standard Specification for Type K and Type N Mineral-Insulated, Metal-Sheathed Thermocouples for Nuclear or for Other High-Reliability Applications", Active Standard ASTM E235 / E235 M, Books of Standards Volume: 14.03

[8] H.K. Jenssen, B.C. Oberländer, J.D. Beenhouwer, J. Sijbers, M. Verwerft, " Neutron radiography and tomography applied to fuel degradation during ramp tests and loss of coolant accident tests in a research reactor", Progress in Nuclear Energy, Volume 72, April 2014, Pages 55-62

[9] F. Nagase, "Behavior of LWR Fuel During Loss-of-Coolant Accidents", Comprehensive Nuclear Materials, 2012, Pages 595-608

[10] Patrick A.C. Raynaud, "Fuel Fragmentation, Relocation and Dispersal During the Loss-of-coolant Accident", NUREG-2121, US NRC 2012.

[11] V. Di Marcello, A. Schubert, J. van de Laar, P. Van Uffelen, "The TRANSURANUS mechanical model for large strain analysis", Nuclear Engineering and Design, Volume 276, September 2014, Pages 19-29

[12] "Benchmark Calculations on Halden IFA-650 LOCA Test Results", NEA/CSNI/R(2010)6

[13] J.X. Li, X.W. Cao, L.L> Tong, G.F. Huang, "Radiological consequence evaluation of DBAs with alternative source term method for a Chinese PWR", Nuclear Engineering and Design, Volume 250, September 2012, Pages 260-266

[14] C.B. Lee. Y. S. Yang, D. H. Kim, S.K. Kim, J.G. Bang, "A new mechanistic and engineering fission gas release model for a uranium dioxide fuel", Journal of Nuclear Science and Technology, Vol.45, No. 1, p. 60-71 (2008)

[15] M. Mogensen, J.H. Pearce, C.T. Walker, "Behaviour of fission gas in the rim region of high burnup $\mathrm{UO}_{2}$ fuel pellets with particular reference to results from an XRF investigation", Journal of Nuclear Materials, Volume 264, Issues 1-2, January 1999, Pages 99-112

[16] G, Pastore, L. Luzzi, V. Di Marcello, P.V. Uffelen, "Physics-based modeling of fission gas swelling and release in $\mathrm{UO}_{2}$ applied to integral fuel rod analysis", Nuclear Engineering and Design, Volume 256, March 2013, Pages 75-86 
[17] S. Holcombe, "Nondestructive Fission Gas Measurements by Means of Gamma Spectroscopy and Gamma Tomography", Licentiate Thesis, Division of Applied Nuclear Physics 2012, Uppsala University

[18] S. Holcombe, S. Jacobsson Svärd, K. Eitrheim, L. Hallstadius, C. Willman, "A Method for Analyzing Fission Gas Release Based on Gamma-ray Measurements", Nuclear Technology, Volume 184, Number 1, Pages 96-106, October 2013

[19] A.A. Harms, D.R. Wyman, "Mathematics and Physics of Neutron Radiography", Springer Science \& Business Media, Mar 31, 1986 


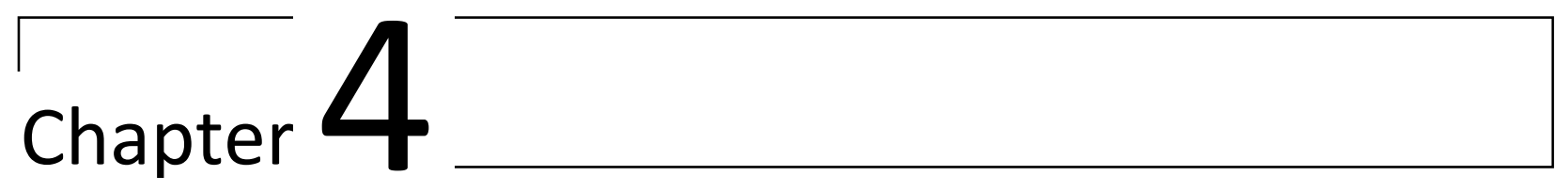

\section{Fragment Size Distribution Correlation}

\section{Contents}

4 Fragment Size Distribution Correlation 116

4.1 Fractal Analysis 117

4.1.1 Theory .118

4.1.2 Proposed Correlation. 124

4.2 Fractal Law Coefficients 128

4.3 Validation of the Fractal Law 131

Bibliography . 136

The experimental investigation on fuel fragmentation has been accompanied by the effort to scientifically describe its mechanism and the resulting fragments' distribution. The first obstacle comes from the latter: what geometrical figure describes the fragments? L.Peliti and A.Vulpiani in the Preface of the Italian edition of "Fractals: Form, Chance and Dimension" [1] underlined that we have learned since Galileo to describe the book of Nature as written in mathematical language of which the typefaces are triangles, circles and other geometrical figures. However, none of the regular and harmonious geometrical figures can rigorously describe the fragments observed as a result of a LOCA Test.

However, dealing with objects that cannot be intuitively treated and connected to a mathematical scheme is not a justification for not treating them scientifically. Our interest is therefore initially in the qualitative aspects of the phenomenon. The first contribution does not have as focus the demonstration of theorems, but it is an idea: fuel fragments exhibiting a fractal dimension: a fractal set that displays as described by Mandelbrot [2] a self-similar patterns, which also means that they are "the same from near as from far".

The first part of this Chapter (4.1) is focused on finding evidence to describe the fragmentation mechanism as a fractal set as per Mandelbrot [2]. Previous studies on the fracture mechanism behavior of concrete performed by Carpinteri and Pugno [3, 4, and 5] are attempts to apply the fractal law to describe a fragmentation mechanism during a transient action. The work of Carpinteri and Pugno is a starting point for the development of a set of fractal laws to describe fuel fragmentation during LOCA. 
The derived Fractal Law contains fractal law coefficients that need to be determined based on the experimental results and the current understanding of the fragmentation phenomena. The objective of this part of the investigation is to create a link between the current knowledge on fuel performance during accidents and the idea of describing the phenomenon with a fractal law set.

The fractal law coefficients are discussed in 4.2 while 4.3 provides a validation of the correlations against the experimental results of the separate effect test and of other LOCA experiments.

\subsection{Fractal Analysis}

A good starting point for this Chapter is the definition of two words: the Latin objective Fractus and the word Fragmentation that has been broadly used in this dissertation. The Latin adjective Fractus means interrupted, irregular and observing the fuel fragments resulting from the LOCA test of Figure 4.1, it is a good attribute. Fragmentation can be defined as the process of breaking apart into fragments caused by the propagation of multiple fractures at different length scales.

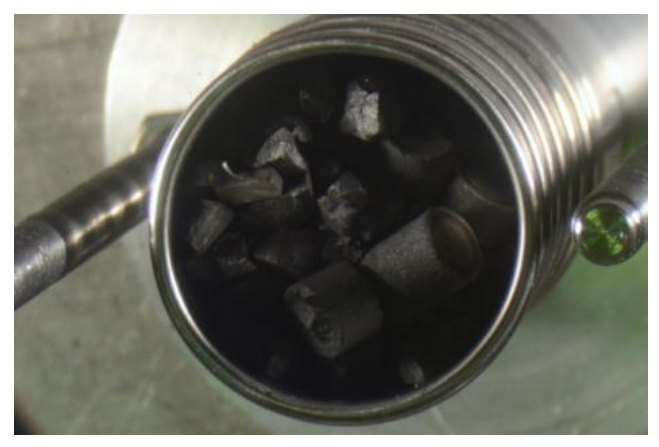

Figure 4.1: Rod 2 fuel fragments during sifting operation Figure taken from sifting operation of Rod 2 (see Figure 3.36)

There have been several attempts to use the fractal theory for modeling of fragmentation processes. The work of Carpinteri and Pugno on the study of fracture behavior of concrete under compression [3, 4, and 5] was mentioned. Other applications of the theory in other natural phenomena are:

- Fractal models for the fragmentation of rocks and soils $[7,8,9]$

- D. L. Turcotte found applications in geology and geophysics [10]

- Sammis found applications in studying granular materials [11]

- Moreover, Takayasu provided a good summary of the different applications of fractal in natural science [12].

Mandelbrot described fractals as hierarchical, often highly irregular, geometrical systems generated using iterative algorithms with relatively simple scaling rules. As a result, no matter how complex a particular spatial pattern might be, the statistical properties of this pattern can be reproduced at other length scales. These scale-invariant systems offer a good opportunity for modeling the propagation of multiple fractures as shown in the post experiment examination of a LOCA test. 
The attempt of using a fractal law to describe the phenomena was thought before the results of the separate effects test were available. Consequently, this approach was initially explored with a benchmark of the experimental results of the NRC test at Studsvik [6].

\subsubsection{Theory}

As a starting point of the investigation, the work of Carpinteri and Pugno [3] has been deeply studied. The work is an extensive experimental investigation on concrete specimens under crushing and fragmentation over a large scale range to evaluate the influence of fragment size distribution on energy density dissipation and related size effect. The experimental fragment analysis performed suggested a fractal law for the distribution in particle size; this simply means that fragments derived from a given specimen appear geometrically self-similar at each observation scale [3]. As example the fragments derived from crushing are represented in Figure 4.2.

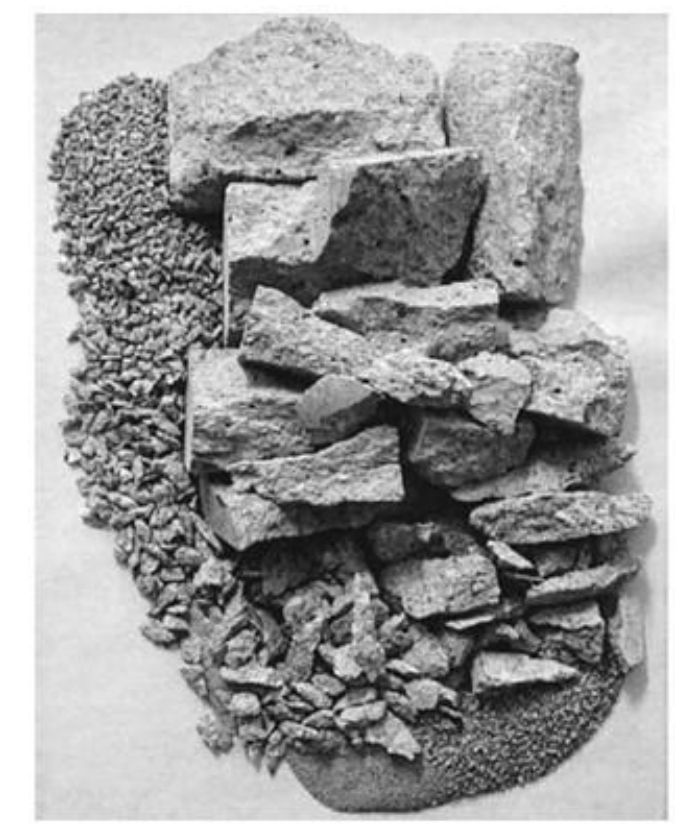

Figure 4.2: Concrete fragments derived from crushing Figure from Reference [3]

Carpinteri and Pugno focused the attention also on the dissipated energy during the event. In fact, the fractal concepts permit to quantify the correlation between fragment size distribution and size effect on dissipated energy, the latter being governed by the total surface area of produced fragments [3].

The approach followed in $[3,4]$ is based on the two following assumptions: 
1. The fragment size distribution and size effect seems to be function of the dissipated energy during the process.

2. Fragments derived from a given specimen appear geometrically similar.

Applying the method for fuel fragmentation during LOCA requires therefore the verification of the above two assumptions.

To evaluate if the fragment size distribution and size effect are function of the dissipated energy during the process, a deeper definition of "process" is required. In Chapter 2.1 observations on parameters that influence fuel fragmentation have been highlighted: 1) status of the irradiated fuel; 2) Effect of the cladding constraints; 3) Effect of fuel rod burst. In the definition of "process" is therefore important to include these three conditions.

Considering the effects of the irradiation on the fuel pellet, they can be considered as an accumulated energy in the fuel and this energy can be released during the accident: the greater the energy accumulated, the larger the possibility of energy dissipation during the transient and the smaller the fragments' size. The phenomena of accumulation of energy during irradiation can be linked to the amount of fission gas generated and the amount of defects and micro-structural changes that make the fuel ceramic matrix weaker.

During the transient heating up, both the increased pressure of the gas and the thermal stresses in the fuel may induce fuel fragmentation. The cladding attached on the fuel and the inner rod pressure provides constraints to the fuel fragmentation. Consequently, the larger the removal of these constraints, the bigger is the opportunity of energy dissipation.

Concerning the second assumption, Figure 4.3 shows that fuel fragments appear geometrically similar. For this purpose, it should be underlined that any kind of pattern that is composed of "pieces" of many different sizes with similar forms (either statistically or geometrically) may well be fractal [13].

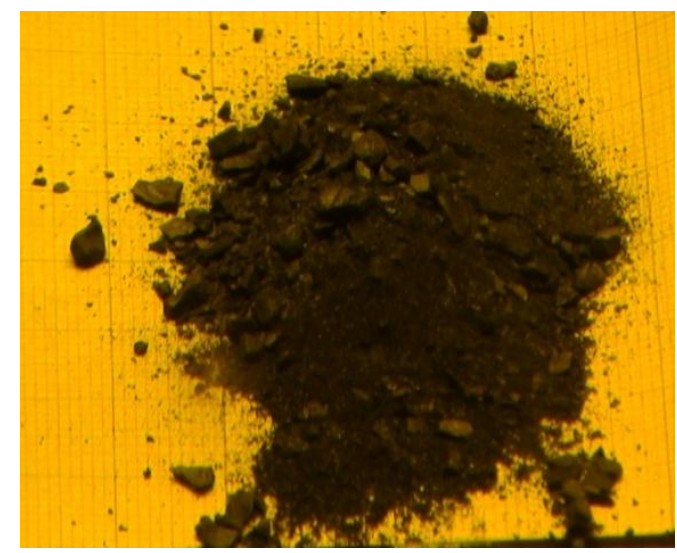

Figure 4.3: Fuel fragments after LOCA - US NRC Test 193 
The two assumptions used by Carpinteri and Pugno [3, 4 and 5] have been qualitatively assessed. The next step embraces a verification of the applicability of the analytical methods of $[3,4$ and 5$]$ to fuel fragmentation during LOCA based on experimental data.

Following concrete's crushing, the cumulative distribution of particles $(p)$ with radius smaller than $r_{f}$ is the following [5]:

$$
p\left(<r_{f}\right)=1-\left(\frac{r_{f, \min }}{r_{f}}\right)^{D}
$$

[Formula 4.1]

Where $r_{f, \min }$ is the smallest fragment size $(\neq 0)$ and the $D$ is the so called fractal dimension. The fractal dimension was introduced by Mandelbrot [1,2] and it can be defined as a measure of the level of irregularity and interruption of the object (consistently with the definition of fractus). Unlike the "normal dimensions", the fractal dimension can be a basic fraction, such as $1 / 2$ or $5 / 3$, or even an irrational number, such as $\frac{\log 4}{\log 3} \sim 1,2618$.. or even $\pi$. Usually, the fractal dimension of a non regular planar curve is between 1 and 2, the one of some surfaces full of convolutions is between 2 and 3 and ultimately powders have a fractal dimension between 0 and 1 . Studying the fragmentation of concrete (result of crushing shown as example in Figure 4.2), Carpinteri and Pugno used values between 2 and 3 depending on the material (e.g. artificially crushed quartz $D=1.89$, disaggregated gneiss $D=2.13$, disaggregated granite $D=2.22$, broken coal $D=2.50$ ).

The Formula 4.1 is valid under the following boundary conditions [3]:

$$
\begin{array}{ll}
p\left(<r_{f, \min }\right)=0 & \text { [Formula 4.2] } \\
p\left(<r_{f, \max }\right) \cong 1 & {[\text { Formula 4.3] }}
\end{array}
$$

The first boundary condition corresponds to a definition of a minimum dimension while the second one is a definition of a maximum dimension.

The density of particles with radius comprised between $r_{f}$ and $r_{f}+d r_{f}$ can be obtained from Formula 4.1 as follow:

$$
p\left(r_{f}\right)=\frac{d p\left(<r_{f}\right)}{d r_{f}}=D \frac{r_{f, \min }^{D}}{r_{f}^{D+1}} \quad[\text { Formula 4.4] }
$$

Even more attractive, is the partial mass of particles below a certain dimension $r_{f}$ as function of the total mass: this is exactly the figure of merit obtained with the fuel particle sifting plot in Figure 3.37. To obtain this ratio, the partial mass and the total mass need to be calculated and the starting point is the evaluation of the volume of the total mass.

$$
V=\int_{r_{f, \min }}^{r_{f, \max }} N_{p}\left(\frac{4}{3} \pi r_{f}^{3}\right) p\left(r_{f}\right) d r_{f}
$$




$$
\begin{gathered}
=\frac{4}{3} \pi N_{p} \frac{D}{3-D} r_{f, \min }^{D}\left(r_{f, \max }^{3-D}-r_{f, \min }^{3-D}\right) \\
\cong \frac{4}{3} \pi N_{p} \frac{D}{3-D} r_{f, \min }^{D} r_{f, \max }^{3-D} \quad \text { [Formula 4.5] }
\end{gathered}
$$

Considering an average density $\rho_{m}$, the total mass $(M)$ and the mass of particles below a certain size $r_{f}$ can be calculated as follow:

$$
\begin{array}{ll}
M \cong \frac{4}{3} \pi N_{p} \rho_{m} \frac{D}{3-D} r_{f, \min }^{D} r_{f, \max }^{3-D} & \text { [Formula 4.6] } \\
M\left(<r_{f}\right) \cong \frac{4}{3} \pi N_{p} \rho_{m} \frac{D}{3-D} r_{f, \min }^{D} r_{f}^{3-D} & \text { [Formula 4.7] }
\end{array}
$$

and the ratio of the two masses:

$$
\frac{M\left(<r_{f}\right)}{M}=\left(\frac{r_{f}}{r_{f, \max }}\right)^{3-D} \quad \text { [Formula 4.8] }
$$

Formula 4.8 is the one used by Carpinteri and Pugno in their investigation and its applicability with fuel fragments was proved against the NRC experimental data from the Studsvik test [6]. The fuel fragment distribution data from [6] are shown in Figure 4.4 and the data are elaborated in Table 4.1. Additional information on the NRC Studsvik test is provided in 1.2.

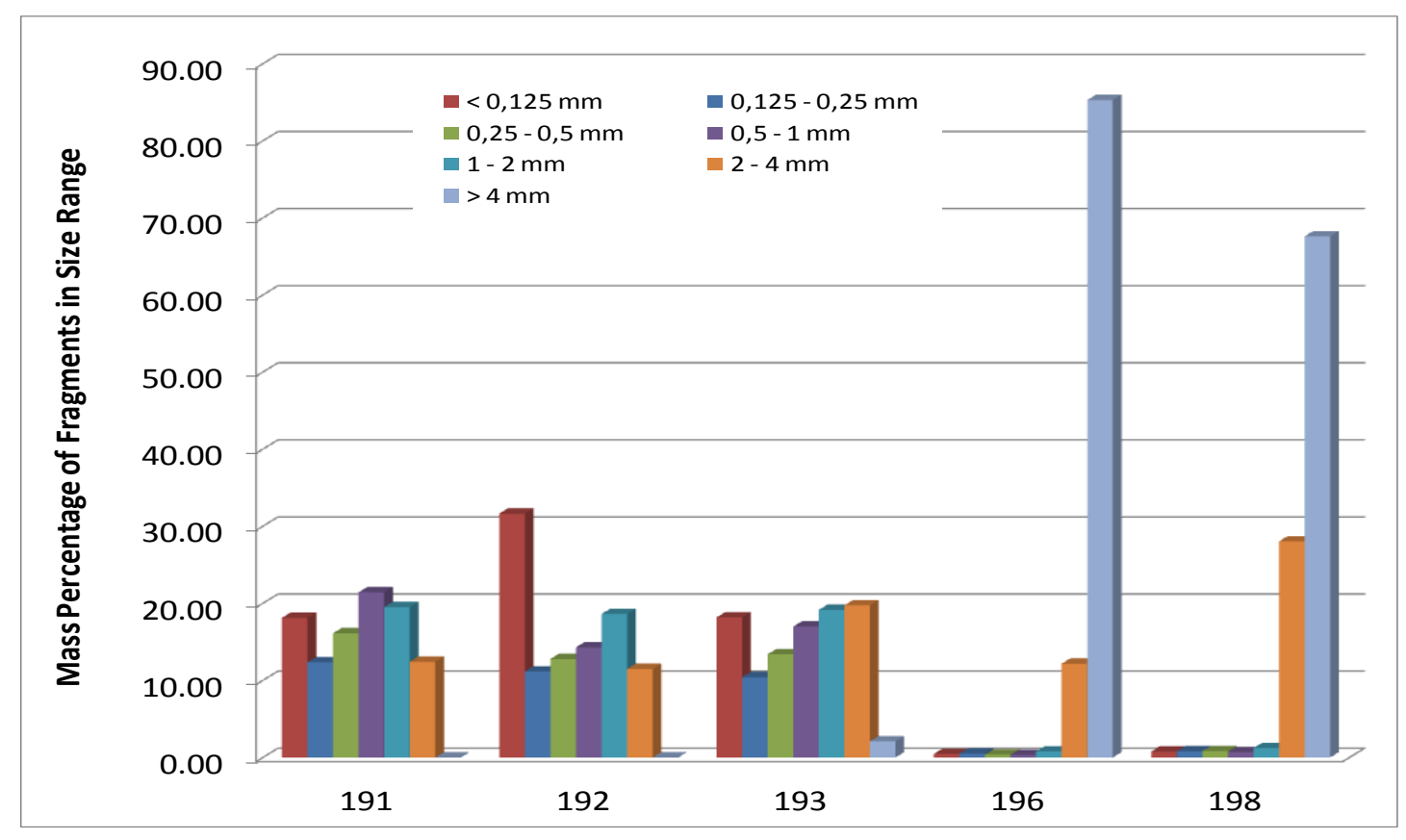

Figure 4.4: Fragmentation size distribution for NRC tests at Studsvik 


\begin{tabular}{|c|c|c|c|}
\hline \multicolumn{2}{|c|}{ Data from Figure 4.4 } & \multicolumn{2}{c|}{ Data Elaborated for the Fractal law } \\
\hline Size Interval [mm] & Mass Percentage [\%] & $\boldsymbol{r}_{\boldsymbol{f}}[\mathbf{m m}]$ & $\boldsymbol{M}\left(<\boldsymbol{r}_{\boldsymbol{f}}\right)[\boldsymbol{\%}]$ \\
\hline $0-0,125$ & 18 & 0,125 & 18 \\
\hline $0,125-0,25$ & 13 & 0,250 & 31 \\
\hline $0,25-0,50$ & 16 & 0,500 & 47 \\
\hline $0,50-1,0$ & 21 & 1,000 & 68 \\
\hline $1,0-2,0$ & 19 & 2,000 & 87 \\
\hline $2,0-4,0$ & 13 & 4,000 & 100 \\
\hline
\end{tabular}

Table 4.1: Fragmentation size distribution for NRC test 191

Formula 4.8 requires the determination of the parameters $r_{f, \max }$ and $D$ :

- as a maximum size dimension the last value of Table $4.1\left(r_{f}=4,0 \mathrm{~mm}\right)$

- a value of $D$ of 2.65 can be preliminary used based on the description of the fractal coefficient of above

The benchmark between the experimental data and the fractal law is given in Figure 4.5. The fractal law seems to follow the fragment size distribution even though the resolution of the correlation can be improved and the uncertainties assessed.

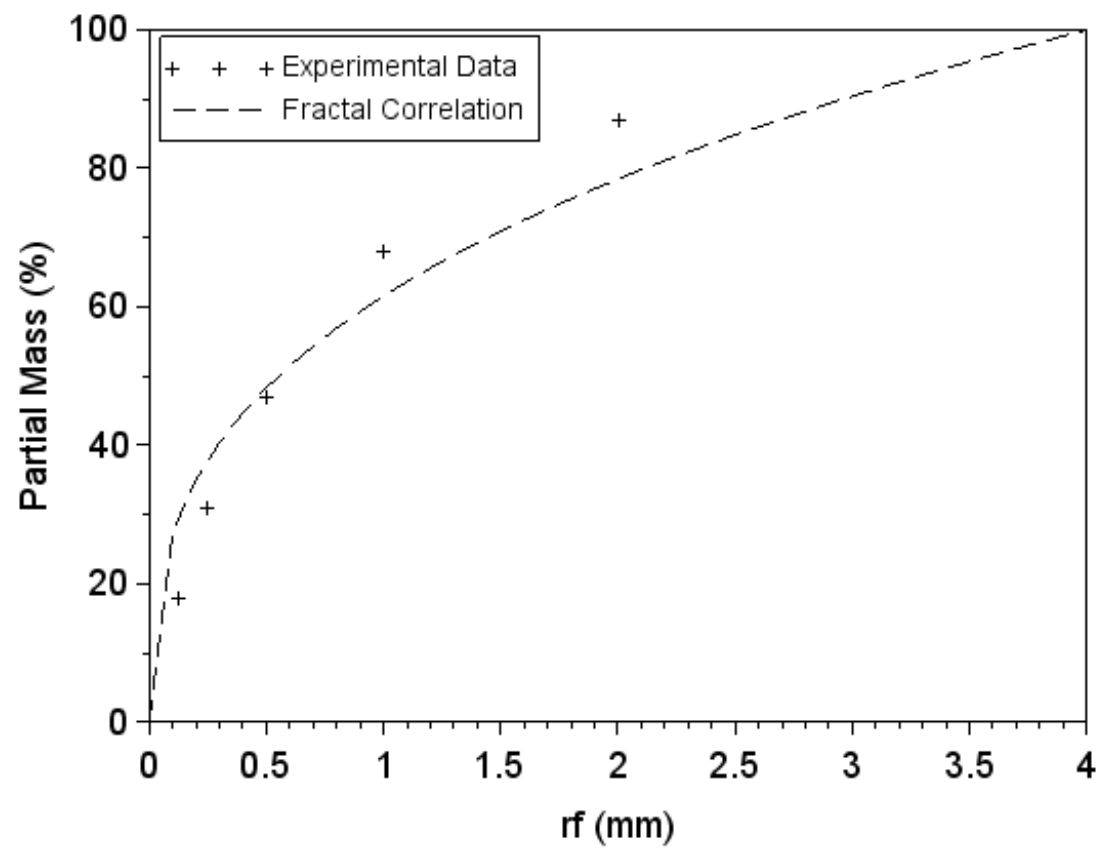

Figure 4.5: Benchmark of Fractal Law against NRC test 191 
Another attempt can be made benchmarking test 198 that has a completely different fragment size distribution because of the smaller burnup (test $19172 \mathrm{MWd} / \mathrm{kgU}$, test $19855 \mathrm{MWd} / \mathrm{kgU}$ ). Table 4.2 was built similarly to Table 4.1 according to the Data of Figure 4.4.

The parameters $r_{f, \max }$ and $D$ for Formula 4.8 were determined as follows:

- as a maximum size dimension the last value of Table $4.1\left(r_{f}=8,0 \mathrm{~mm}\right)$

- The value of $D$ was iteratively reduced up to a value of 1.3 that seems appropriate to benchmark the experiment data.

\begin{tabular}{|c|c|c|c|}
\hline \multicolumn{2}{|c|}{ Data from Figure 4.4 } & \multicolumn{2}{c|}{ Data Elaborated for the Fractal law } \\
\hline Size Interval [mm] & Mass Percentage [\%] & $\boldsymbol{r}_{\boldsymbol{f}}[\mathbf{m m}]$ & $\boldsymbol{M}\left(<\boldsymbol{r}_{\boldsymbol{f}}\right)[\boldsymbol{\%}]$ \\
\hline $0-0,125$ & 1 & 0,125 & 2 \\
\hline $0,125-0,25$ & 1 & 0,250 & 3 \\
\hline $0,25-0,50$ & 1 & 0,500 & 4 \\
\hline $0,50-1,0$ & 1 & 1,000 & 6 \\
\hline $1,0-2,0$ & 2 & 2,000 & 34 \\
\hline $2,0-4,0$ & 28 & 4,000 & 100 \\
\hline 8,0 & 67 & 8,000 & 2 \\
\hline
\end{tabular}

Table 4.2: Fragmentation size distribution for NRC tests 198

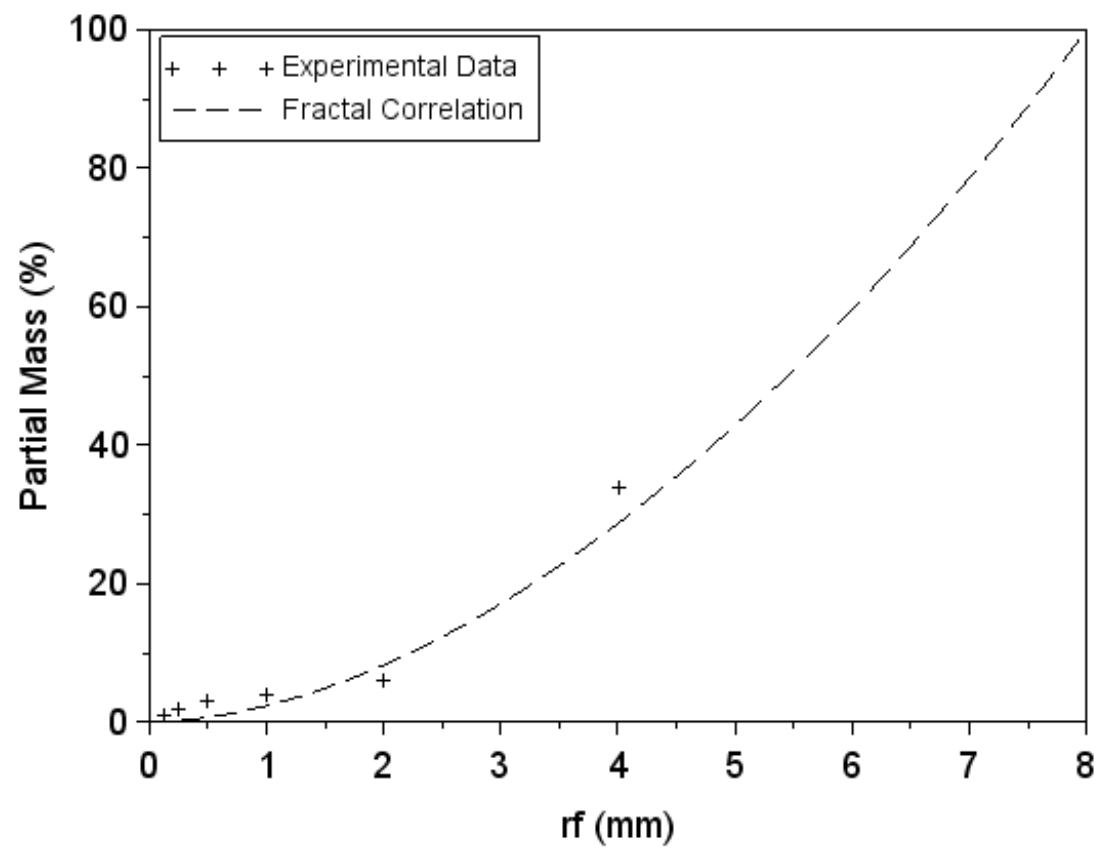

Figure 4.6: Benchmark of Fractal Law against NRC test 198 
The fractal law used by Carpinteri and Pugno seems to sufficiently describe the fragment size distribution of the test 191 but the benchmark with the test 198 (Figure 4.6) shows weaknesses. In particular, for small values of $r_{f}$, the concavity of the fractal law is the opposite of what the experimental data are showing and moreover, a value of the fractal coefficient smaller than 2 was needed while instead, given the dimension and shape of the fragments, a value bigger than 2 is expected.

The next Chapter proposes, starting from the work of Carpinteri and Pugno, a different approach that can describe with "more" accuracy fuel fragmentation during LOCA.

\subsubsection{Proposed Correlation}

The proposed approach to describe fuel fragmentation during LOCA is based on the introduction of the concept of superposition of fractal laws. The superposition of fractal laws is considered more appropriate because there are two main differences between the fragmentation described by Carpinteri and Pugno and the one observed during a LOCA:

- The fuel structure of an irradiated fuel rod is not homogeneous: In Chapter 2.1.1 the transformation occurring in the fuel during irradiation and the possibility of having different fuel properties at different radial locations are described.

- The external constraints to fuel fragmentation evolve during the transient: Adopting the Mandelbrot fractal mechanism, we are conceiving the fragmentation occurring in the fuel as a multiple process involving many subsequent fragmentation stages which produce smaller particles at each subsequent stage. Chapter 2.1.1 introduced the concept of two constraints acting as resistance forces to fuel fragmentation. Owing to different relocation opportunities of the fuel fragments after consecutive fragmentation steps, the dynamics of the competitive forces can change as function of the evolution of the external constraints (geometrical of the cladding and internal rod pressure) during the transient.

The argumentations of above suggest the use of a superposition of different fractal laws to consider the different properties of the fuel pellet and the potential different propagation of the fragmentation steps.

As a first step of the investigation, the superposition of two fractal laws is derived. The two fractal laws apply to two different partial masses of the fuel. The total mass of the fuel is therefore divided in two masses based on similar fuel structure and similar competitive fragmentation forces through different successive steps of fuel fragmentation. The idea that two fractal laws may be sufficient to describe the phenomenon comes also from studying the experimental data of test 191 and 198 in a logarithmic plot (Figure 4.7 and Figure 4.8). On the logarithmic scale, a fractal law as described by Formula 4.8 is a straight line and for both the experiments it is noticeable that two straight lines are needed to increase the resolution of the correlation against the experimental data. 


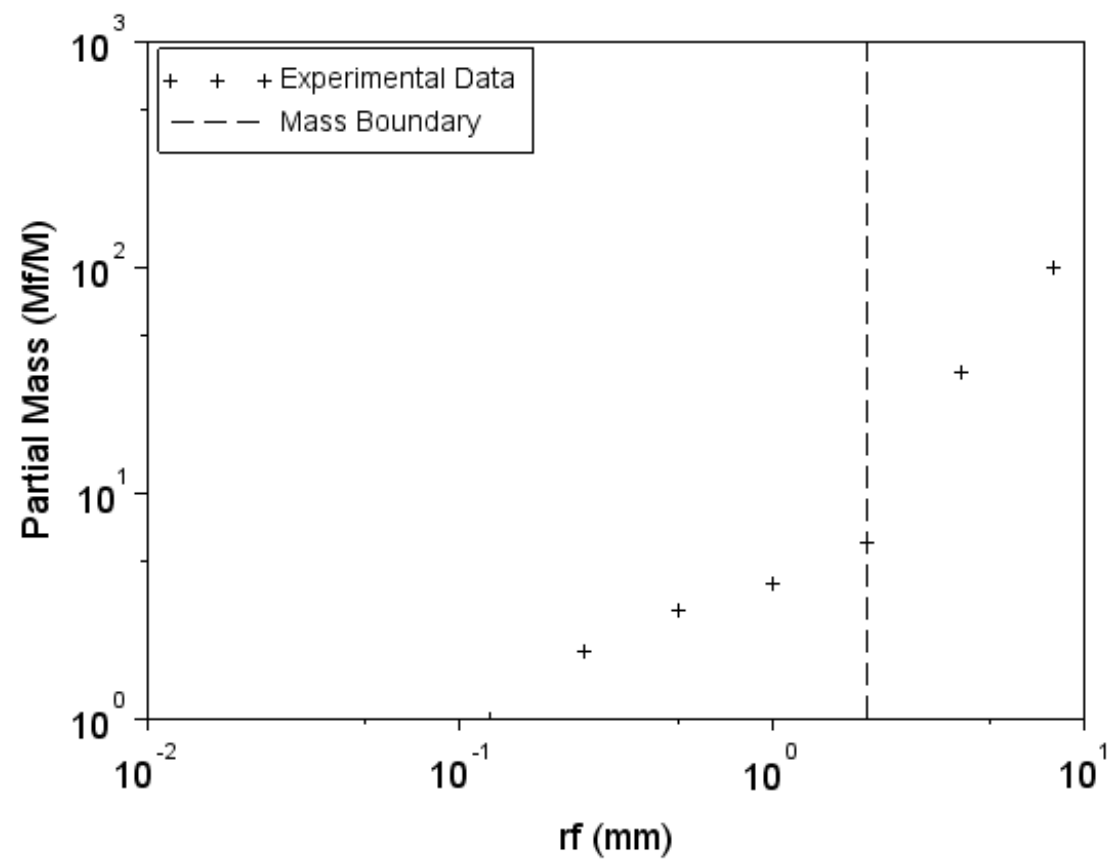

Figure 4.7: Cumulative partial mass for US NRC test 191

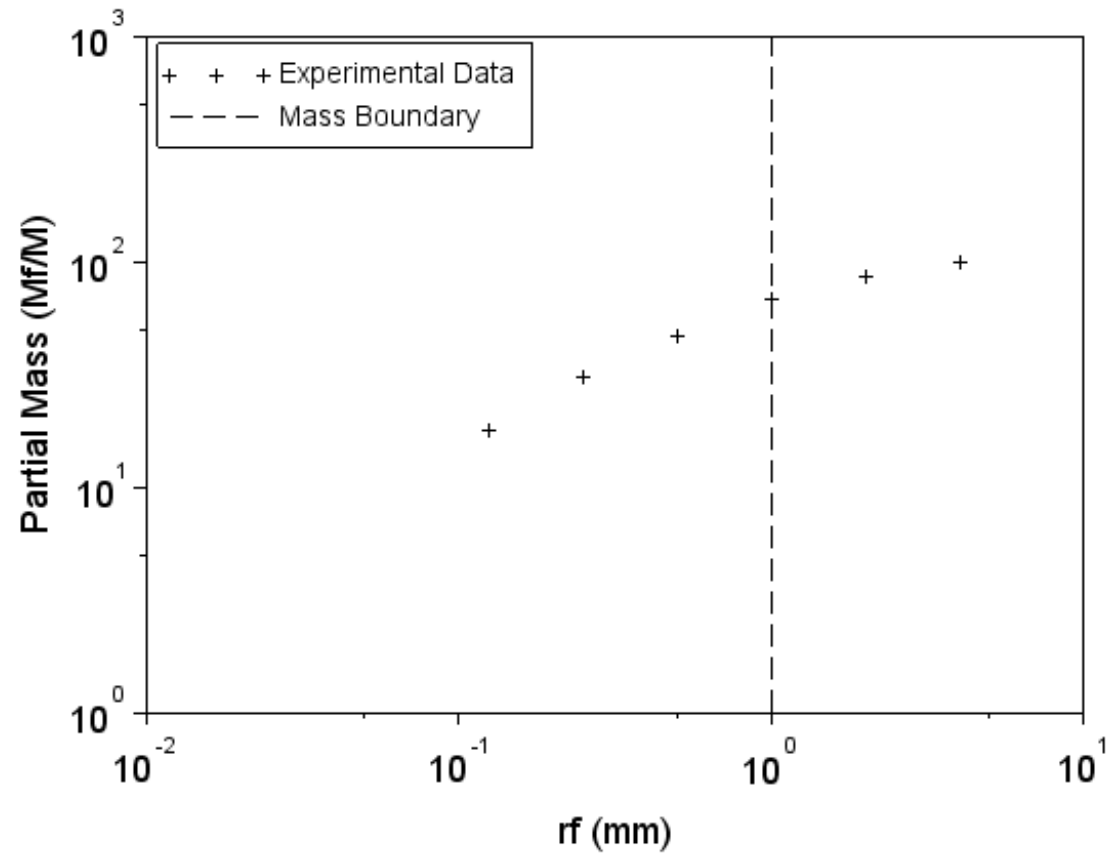

Figure 4.8: Cumulative partial mass for US NRC test 198 
The two fractal laws should cover the whole spectrum of potential fragment size dimension between powder $(\sim 0)$ and $r_{f, \max }$. In particular, the first one is applicable between $r_{f, \min 1}$ and $r_{f, \max 1}=r_{f, \max }$, while the second one between 0 and $r_{f, \max 2}=r_{f, \min 1}$. As they are built, the two laws apply to two separated but consecutive fragment sizes regions and they correspond to two partial masses of which the sum is equal to the total mass of the fuel.

The calculation of the partial mass $M_{1}$ (Formula 4.9) can be obtained with similar steps used to derive Formula 4.8 starting from the volume Formula 4.5. Formula 4.10 shows the mass amount of particles of $M_{1}$ with sizes smaller than $r_{f}$ and Formula 4.11 the ratio between the latter and $M_{1}$.

$$
\begin{gathered}
M_{1}=\rho_{m} \frac{4}{3} \pi N_{p} \frac{D_{1}}{3-D_{1}} r_{f, \min 1}^{D_{1}}\left(r_{f, \max 1}^{3-D_{1}}-r_{f, \min 1}^{3-D_{1}}\right) \\
M_{1}\left(<r_{f}\right)=\rho_{m} \frac{4}{3} \pi N_{p} \frac{D_{1}}{3-D_{1}} r_{f, \min 1}^{D_{1}}\left(r_{f}^{3-D_{1}}-r_{f, \min 1}^{3-D_{1}}\right) \\
\frac{M_{1}\left(<r_{f}\right)}{M_{1}}=\frac{r_{f}^{3-D_{1}}-r_{f, \min 1}^{3-D_{1}}}{r_{f, \max 1}^{3-D_{1}}-r_{f, \min 1}^{3-D_{1}}} \quad \text { (Form }
\end{gathered}
$$

A similar ratio for the partial mass $M_{2}$ can be obtained directly from Formula 4.8:

$$
\frac{M_{2}\left(<r_{f}\right)}{M_{2}}=\left(\frac{r_{f}}{r_{f, \max 2}}\right)^{3-D_{2}}
$$

Considering as a total mass of the fuel $M$, the following Formulas can be written considering the partial mass ratio $K(0<K<1)$ :

$$
\begin{gathered}
\frac{M_{2}}{M}=K \rightarrow M_{2}=K \cdot M \\
\frac{M_{1}}{M}=1-K \rightarrow M_{1}=(1-K) \cdot M
\end{gathered}
$$

Replacing Formula 4.13 in Formula 4.11 and Formula 4.14 in Formula 4.1:

$$
\begin{gathered}
\frac{M_{1}\left(<r_{f}\right)}{M}=(1-K) \frac{r_{f}^{3-D_{1}}-r_{f, \min 1}^{3-D_{1}}}{r_{f, \max 1}^{3-D_{1}}-r_{f, \min 1}^{3-D_{1}}} \\
\frac{M_{2}\left(<r_{f}\right)}{M}=K\left(\frac{r_{f}}{r_{f, \max 2}}\right)^{3-D_{2}}
\end{gathered}
$$

Considering that $r_{f, \max 2}=r_{f, \min 1}$ and that the two fractal laws apply to two separated but consecutive fragment sizes regions, the two laws can be lump in Formula 4.17:

$$
\frac{M\left(<r_{f}\right)}{M}=u_{1} \cdot\left[(1-K) \frac{r_{f}^{3-D_{1}}-r_{f, \min 1}^{3-D_{1}}}{r_{f, \max 1}^{3-D_{1}}-r_{f, \min 1}^{3-D_{1}}}+K\right]+u_{2} \cdot K\left(\frac{r_{f}}{r_{f, \min 1}}\right)^{3-D_{2}}
$$


Where:

$u_{1}=\left\{\begin{array}{l}1 \rightarrow r_{\mathrm{f}}>r_{\mathrm{f}, \min 1} \\ 0 \rightarrow \mathrm{r}_{\mathrm{f}}<\mathrm{r}_{\mathrm{f}, \min 1}\end{array} \quad u_{2}=\left\{\begin{array}{l}1 \rightarrow \mathrm{r}_{\mathrm{f}}<\mathrm{r}_{\mathrm{f}, \min 1} \\ 0 \rightarrow \mathrm{r}_{\mathrm{f}}>\mathrm{r}_{\mathrm{f}, \min 1}\end{array}\right.\right.$

Figure 4.9 shows the benchmark of Formula 4.17 against the experimental data of tests 191 and 198 with the coefficients determined with an iterative process to fit the experimental data.

- $\quad$ Test 191: $D_{1}=2,99 ; D_{2}=2,4 ; r_{f, \min 2}=1,2 ; ; r_{f, \max 2}=4 ; K=0,75$

- Test 198: $D_{1}=2,99 ; D_{2}=2,4 ; r_{f, \min 2}=3 ; r_{f, \max 2}=8 ; K=0,08$
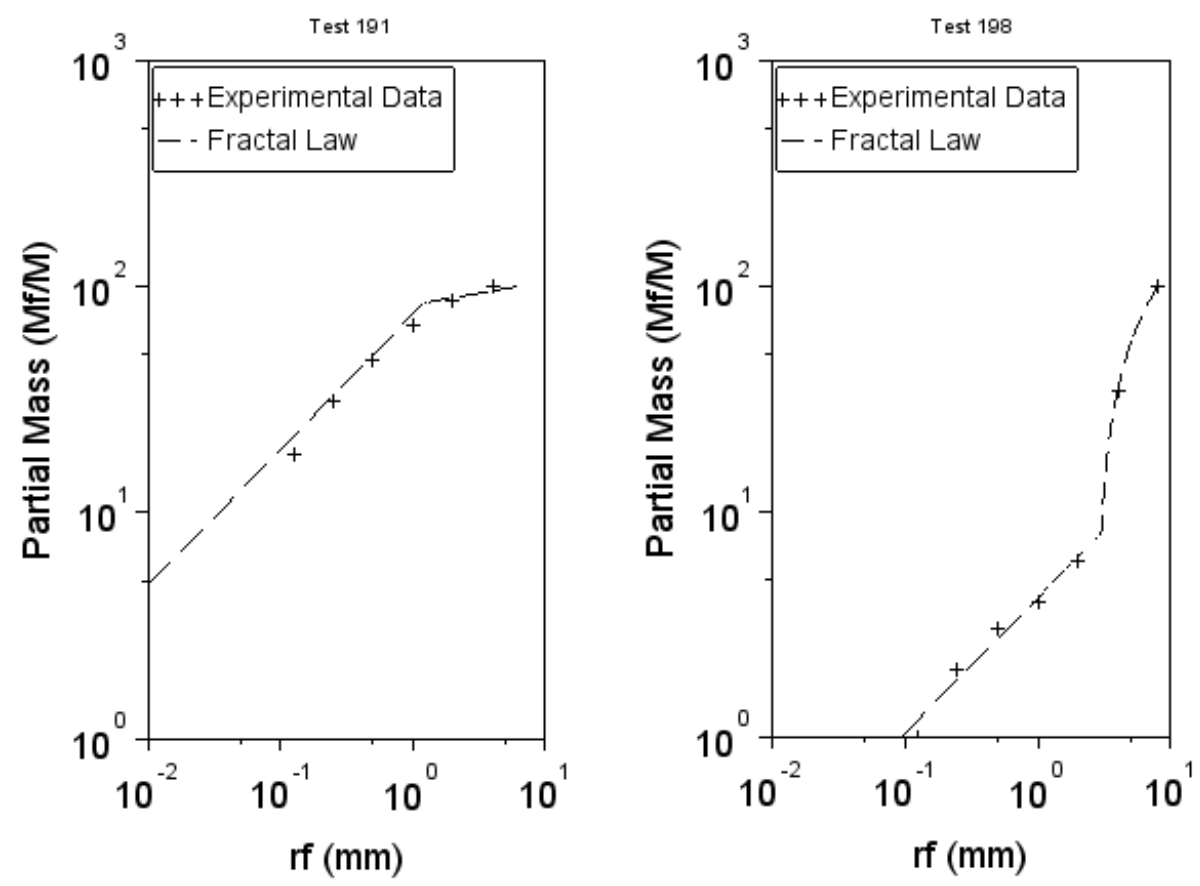

Figure 4.9: Superposition of fractal laws for NRC Studsvik tests Left: NRC Test 191; Right: NRC Test 198

The superposition of two fractal laws has improved the resolution in representing the data of test 191 and solved the inconsistencies found applying the method of Carpinteri and Pugno for test 198. The approach proves to have the flexibility required to describe fragment size distribution occurring after a LOCA test at different burnup. In the next Chapter, the coefficients of Formula 4.17 are discussed. 


\subsection{Fractal Law Coefficients}

Using the Formula 4.17 to represent and/or predict fuel fragmentation during LOCA based on the irradiated fuel segment properties and transient characteristics implies obtaining the coefficients listed below:

- $r_{f, \max 1}$ : This value represents the maximum dimension of the fragments. Fragmentation is a relevant phenomenon in the conventional light water reactor because small fragments can relocate axially within the fuel (in particular in the ballooning region) and determine new axial power distribution and/or may escape from the fuel rod and overstep the defense in depth barrier of the cladding and regulators are looking at the phenomena to determine potential impact on design basic accidents evaluations [14]. Since the mobility of bigger fragments (e.g. half of the diameter of the fuel) is very limited, fuel relocation on the ballooning region and/or fuel dispersal becomes unlikely. This coefficient can be therefore considered as a constant and its value can be fixed at $r_{f, \max 1}=4 \mathrm{~mm}$, because a refined distribution of particles above this value does not provide any valuable information in terms of safety consequences.

- $r_{f, \min 1}=r_{f, \max 2}$ : This value indicates the threshold between the application regions of the two fractal laws and to which extent the partial mass $M_{1}$ will fragment.

- $K$ : to determine the value of $K$, two partial masses need to be classified considering part of the fuel with similar properties and similar transient characteristics.

- $D_{1}$ : The value of this fractal coefficient is found to be constant and equal to 2.9

- $D_{2}$ : The value of this fractal coefficient is found to be constant and equal to 2.4

Considering that the two fractal coefficients $D_{1}$ and $D_{2}$ are defined invariant and the value of $r_{f, \max 1}$ is also a constant, the only two parameters that need to be defined are $r_{f, \min 1}$ and $K$.

This analysis is based on observation on LOCA experimental tests, where short fuel segments have been used. Scaling the analysis to a full length fuel rod implies precaution and the following considerations are needed to be taken into account:

- Status of the irradiated fuel: The irradiation cycles of a fuel rod are very different considering different fuel axial locations. First, the axial power profile changes in time as function of the control rods movements and moderator density. In presence of in-core flux instrumentation as BWR with the Local Power Range Monitor (LPRM) [15] and KWU-Siemens PWR with the Power Density Detectors (PDD), the axial power profile can be determined with several procedures [e.g. 17]. Consequently the axial fuel burnup distribution is influenced by the power history at different axial locations. The work of Tayama [18] shows an experimental procedure to determine the burnup distribution of a BWR fuel rod irradiated up to $60 \mathrm{MWd} / \mathrm{kgU}$ in Japan. The results of the experimental investigation are shown in Figure 4.10. Consequently, the status of the irradiated fuel changes at different axial locations and the correlation can be applied to fuel axial segments with similar fuel properties 
- Removal of the cladding constraint: The degree of removal of the constraint provided by the cladding at different axial locations is function of the heat stored in the fuel rod at different axial locations at the time of LOCA. Different heat stored values correspond to different values of decay heat to be removed from the fuel rod [19]. The bigger the decay heat, the bigger the cladding temperature and the larger the cladding deformation due to creep [20].

- Removal of the constraint of the rod internal pressure: The degree of removal of the constraint provided by the rod internal pressure depends upon the level of pressure from which the burst occurs as a potential energy level that can be released during burst. Additionally, this level of pressure can be different between axial locations of the fuel. In 2.1.3 it has been already shown that with a fuel segment such as the IFA-650 $(\approx 500 \mathrm{~mm})$, the depressurization at the top of the fuel can be slower than at the middle (or at the bottom) burst region where the depressurization is very fast. Consequently, the degree of the removal of the constraint is function of the gas pressure at the time of the burst and the depressurization rate. A dynamic axial gas model is therefore required to take into account these phenomena for full length rod to consider the level of gas communication between different axial regions and to consider also the drop of local pressure in an "isolated" ballooned area. The FALCON-PSI code has implemented a model able to simulate these phenomena [21].

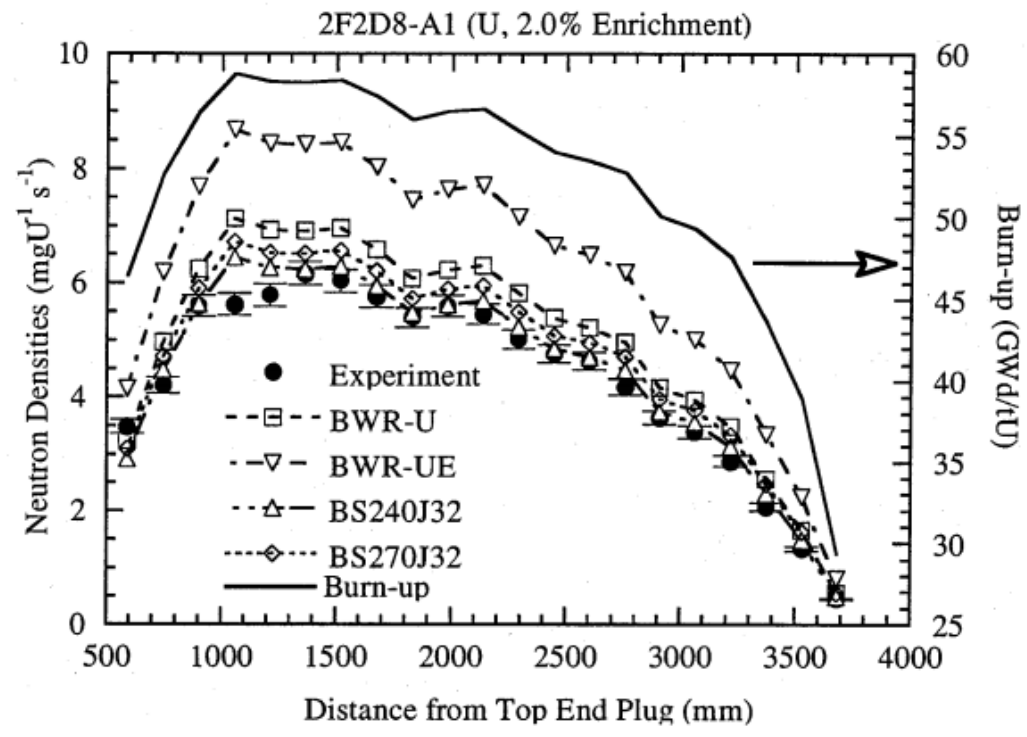

Figure 4.10: Neutron Density and Burnup axial Power Distribution for BWR fuel Figure from Reference [18]

Based on the considerations above, the parameters $r_{f, \min 1}$ and $K$ should be calculated for axial nodes with similar characteristics in terms of fuel properties, ballooning size and if occurs, drop in pressure of the local area due to burst.

The determination of $r_{f, \min 1}$ and $K$ was done considering the observations made on fuel fragmentation of 2.1, the results of the separate effects test of 3.0 and the definition of the two parameters given in 
4.1.2. In particular, based on the classification of 2.1 in fuel cracks, coherent fuel fragmentation and fuel pulverization region, the fuel crack region results of no interest because the fragments have usually a relevant dimension and their relocation is unlikely. In this region, the fuel matrix has not undergone significant micro-structural changes since the HBS has not developed yet. Consequently, based on the Formula $4.13 M_{2}=0$ and consequently $K=0$. The value of $r_{f, \min 1}$ can be chosen arbitrary as $4.0 \mathrm{~mm}$ because in the fuel cracks region most of the fuel seem to crack to a minimum dimension that is slightly smaller than the radius of the pellet. It should be underlined that these parameters in the fuel crack regions could be refined based on the accurate value of fuel burnup and transient boundary conditions but the safety significance of refined values would be irrelevant.

Correlation needs to be found instead for the region of the fuel classified as coherent fuel fragmentation that starts at $40-45 \mathrm{MWd} / \mathrm{kgU}$ and terminates at $70-80 \mathrm{MWd} / \mathrm{kgU}$. This region corresponds to the development of the HBS and its increase of thickness. It should be underlined that the development of the HBS is function of the burnup but also of the irradiation temperature [22].

The value of $r_{f, \min 1}$ represents the boundary between the two fractal law application regions, while $K$ represents the ratio between the mass of the region characterized by smaller fragments and the total mass. Based on this definition the value of $r_{f, \min 1}$ can be considered as a pure function of fuel burnup. For continuity with the fuel cracks region, the starting value of $r_{f, \min 1}$ at $45 \mathrm{MWd} / \mathrm{kgU}$ is equal to $4 \mathrm{~mm}$. The value decreases with fuel burnup and for very high burnup fuel (e.g. IFA-650.4) the fuel fragments become very small. The proposed correlation for $r_{f, \min 1}$ as a function of the fuel burnup $B U$ is valid also for the fuel pulverization region and therefore with a range of applicability between 45 and 100 $\mathrm{MWd} / \mathrm{kgU}$.

$$
r_{f, \min 1}=0.0015455 \cdot B U^{2}-0.2877273 \cdot B U+13.818182 \quad[\text { Formula 4.19] }
$$

The determination of the value of $K$ is in line with the separation of the effects discussed in the dissertation and studied with the separate effects test. The proposed correlation is function of a parameter linked to the current status of the irradiated fuel, to the degree of removal of the geometrical constraint provided by the cladding and the degree of removal of the constraint provided by the rod internal pressure.

$$
K=K_{\max }^{0.7} \cdot\left(\frac{A V G_{\text {strain }}}{K s}\right)^{0.7} \cdot\left(\frac{p_{\text {burst }}}{K p}\right)^{0.4} \quad[\text { Formula 4.20] }
$$

where $A V G_{\text {strain }}$ is the average circumferential strain of the cladding along the axial length and $p_{\text {burst }}$ is the burst pressure. These two variables can be measured during an experiment or calculated with fuel performance codes such as TRANSURANUS [23], FALCON [21], FRAPTRAN [24] and GRS Code [25]. The bigger the value of $A V G_{\text {strain }}$, the larger the removal of the geometrical constraint provided by the cladding. Since after a certain degree of ballooning the effect of further cladding deformation is negligible, a limiting reference value of average cladding strain $K s$ was introduced and a value of 15.0\% was assigned to it. The value of the ratio $\frac{A V G_{\text {strain }}}{K S}$ is limited to 1.0. The use of the average circumferential strain along the length of the cladding considers also the situation of local ballooning. In 
this case, the $A V G_{\text {strain }}$ is limited because the bigger values of the strain of the ballooning region are compensated by the smaller values of the axial zone that did not balloon. Consequently this local and "partial" removal of the constraint provided by the cladding is considered with a reduced value of K.

The bigger the burst pressure, the bigger the removal of the constraint. Similarly to $K s$, a limiting reference value was considered for rod burst $(K p) . K p$ is defined equal to 100 bar and the ratio $\frac{p_{\text {burst }}}{K p}$ is limited to 1.0. If burst does not occur, the situation is considered comparable to a burst occurring at very low pressure (e.g. 10 bar).

The correlation was built based on the following concept: $K_{\max }$ represents a full potential based on the fuel's status and assuming a complete removal of the geometrical constraint provided by the cladding and a complete removal of the constraint provided by the rod internal pressure. These conditions correspond to a scenario with $\frac{A V G_{\text {strain }}}{K s}=\frac{p_{\text {burst }}}{K p}=1$ and $K=K_{\text {max }}$ If the removal of the two constraints is not complete, the values of the two ratios $\frac{A V G_{\text {strain }}}{K s}$ and $\frac{p_{\text {burst }}}{K p}$ are smaller than 1.0 and consequently the value of $K<K_{\max }$.

A correlation is proposed for $K_{\max }$ that is differentiated for the coherent fuel fragmentation and fuel pulverization regions:

$K_{\max }=\left\{\begin{array}{c}0.0010545 \cdot B U^{2}-0.096923 \cdot B U+2.21314 \rightarrow \frac{45 M W d}{k g U}<B U<70 \frac{M W d}{\mathrm{~kg}} \\ 0.0046667 \cdot B U+0.283333 \rightarrow \frac{70 M W d}{\mathrm{kgU}}<B U<100 \frac{\mathrm{MWd}}{\mathrm{kg}}\end{array}\right.$ (Formula 4.21)

The correlation for the fuel coherent fragmentation region has a behavior that is function of the burnup similar to the development of the thickness of the "transient HBS". Although the development of the HBS structure is frozen because the criteria of irradiation temperature is not satisfied, the continuous increase of burnup further creates defects in the fuel and consequently an additional linear increase of the value of $K_{\text {max }}$ is proposed for values of BU above $70 \frac{\mathrm{MWd}}{\mathrm{kg}}$.

The correlations function of fuel burnup have been derived considering the development of the HBS thickness in the fuel pellet during irradiation for a typical LWR fuel (e.g. Figure 2.4).

\subsection{Validation of the Fractal Law}

The proposed correlation described in 4.1.2 and the fractal law coefficients (4.2) were validated against the US NRC Test 191 and Test 198 [6], the IFA-650.4 [26] and the separate effects test Rod 1 and Rod 2. The data needed to be considered in the model are summarized in Table 4.3. Table 4.3 provides also the calculated fractal law coefficients $r_{f, \min 1}$ and $\mathrm{K}$. 
Due to the lack of models in describing the fuel fragmentation and the weakness of fuel performance codes in simulating the phenomenon, the objective of the investigation was an attempt to identify a semi-empirical correlation that can capture the first order parameters that are relevant in the process. The correlation also allows assigning quantitatively contributions to the effects studied in this experimental investigation: the geometrical constraint provided by the cladding and the constraint provided by the rod internal pressure. The determination of the fractal law coefficient $K$ is a function of the average strain of the segment and the burst pressure.

The correlation can be considered as a starting point for further investigations that can encompass other parameters, such as the different heating up rate or the maximum fuel temperature during the transient. The present experiment was conceived as a separate effects test and therefore some variables were fixed by definition. Additionally, the correlation can be implemented in fuel performance codes because it is design to be applied at different axial locations and consequently it is suitable for the case with axial discretization of the fuel rod. Moreover, when new experimental data are available, the formulation of the fractal coefficients and constants can be improved.

The validation against experimental data is shown in Figure 4.11-4.15. Considering the uncertainties of a first order correlation, the validation is considered appropriate.

\begin{tabular}{|l|c|c|c|c|c|}
\hline \multicolumn{1}{|c|}{ Experiment } & $\begin{array}{c}\text { BU } \\
{[\mathbf{M W d / k g U ]}}\end{array}$ & $\begin{array}{c}\text { Average } \\
\text { Circumferential Strain } \\
{[\mathbf{\%}]}\end{array}$ & $\begin{array}{c}\text { Burst Pressure } \\
{[\mathbf{b a r}]}\end{array}$ & $\begin{array}{c}\boldsymbol{r}_{\boldsymbol{f , \boldsymbol { m i n } \mathbf { 1 }}} \\
{[\mathbf{m m}]}\end{array}$ & $\boldsymbol{K}$ \\
\hline US NRC Test 191 & 71 & $15.5^{*}$ & 110 & 1.18 & 0.74 \\
\hline US NRC Test 198 & 55 & $12.0^{*}$ & 74 & 2.67 & 0.13 \\
\hline IFA-650.4 & 92 & 19.4 & 53 & 0.43 & 1 \\
\hline Rod 1 & 52 & 14.0 & No burst** & 3.04 & 0.03 \\
\hline Rod 2 & 52 & 8.0 & $40^{* * *}$ & 3.04 & 0.04 \\
\hline
\end{tabular}

Table 4.3: Experiments' data needed for fractal law model validation

*Estimation; ${ }^{* *}$ Assumed equal to 10 bar in the model; ${ }^{* * *}$ Calculated by TRANSURANUS 


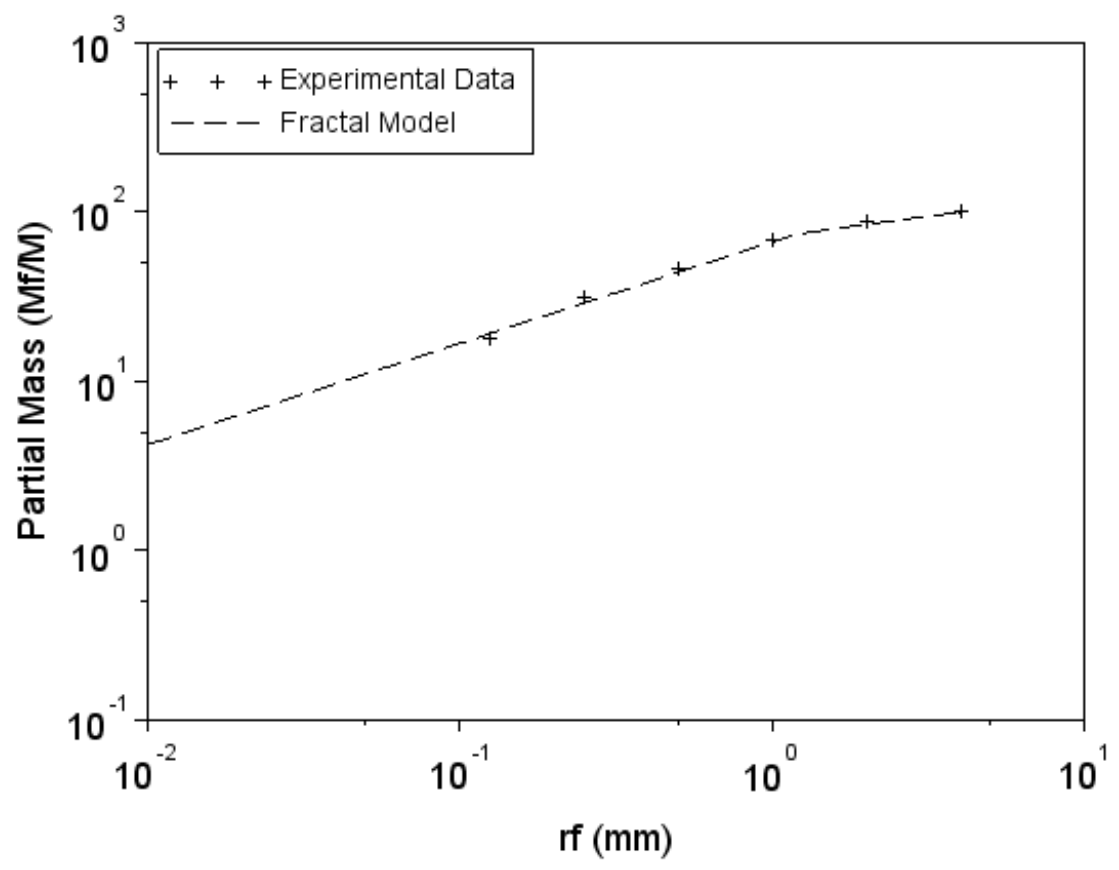

Figure 4.11: Validation US NRC Test 191

The partial mass expresses in percentage (\%) the cumulative mass of particles below $\mathrm{rf}$

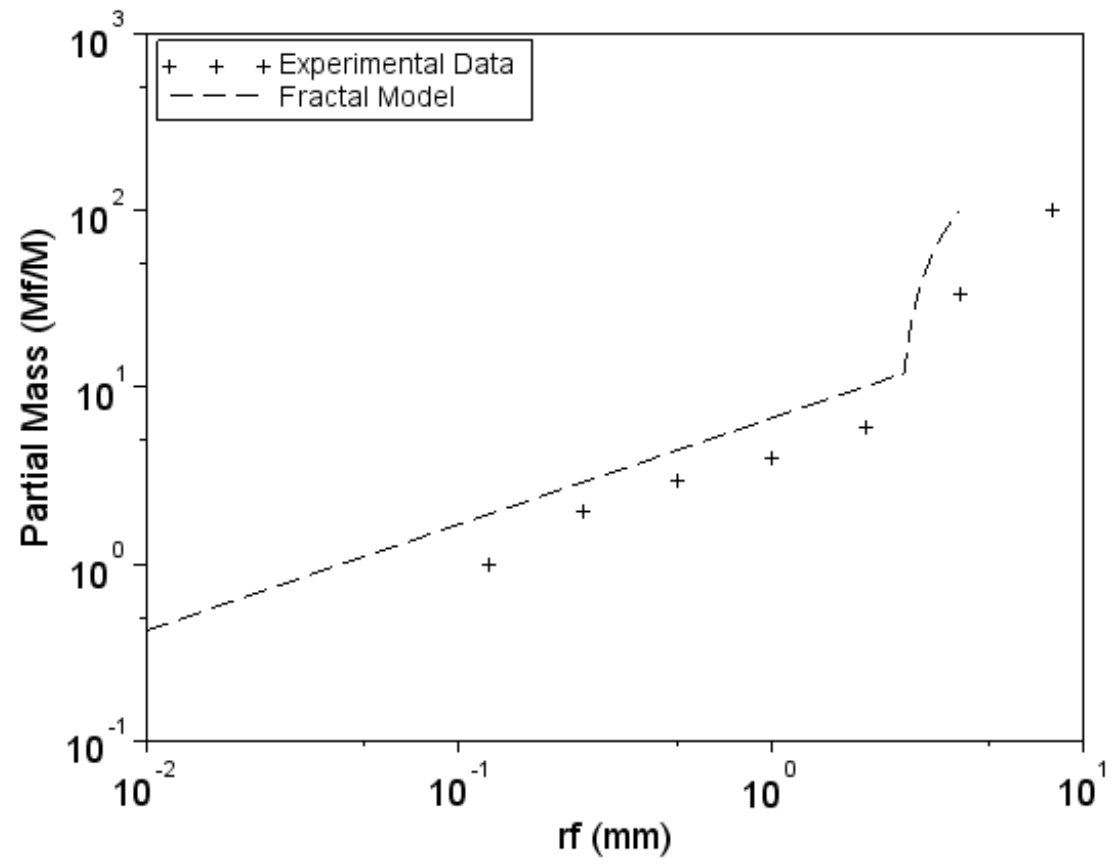

Figure 4.12: Validation US NRC Test 198

The partial mass expresses in percentage (\%) the cumulative mass of particles below $r$ 


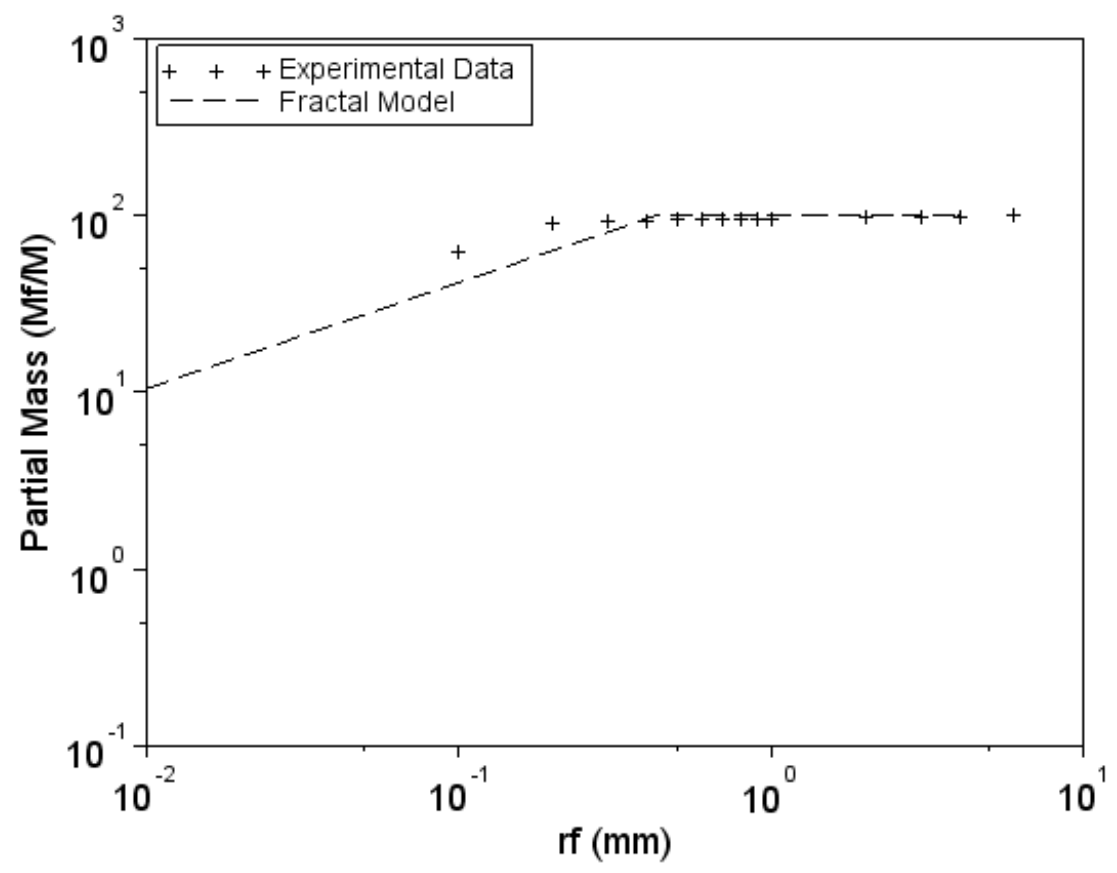

Figure 4.13: Validation IFA-650.4

The partial mass expresses in percentage (\%) the cumulative mass of particles below $\mathrm{rf}$

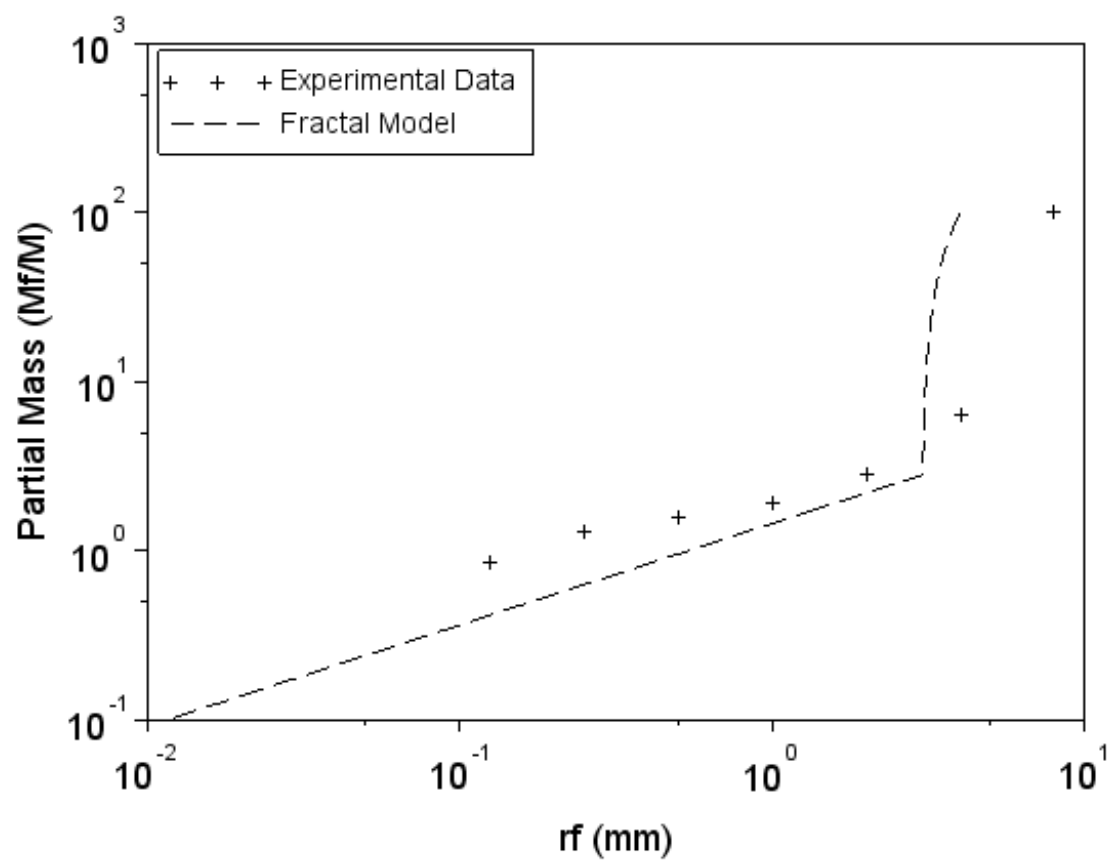

Figure 4.14: Validation separate effects test Rod 1

The partial mass expresses in percentage (\%) the cumulative mass of particles below $\mathrm{rf}$ 


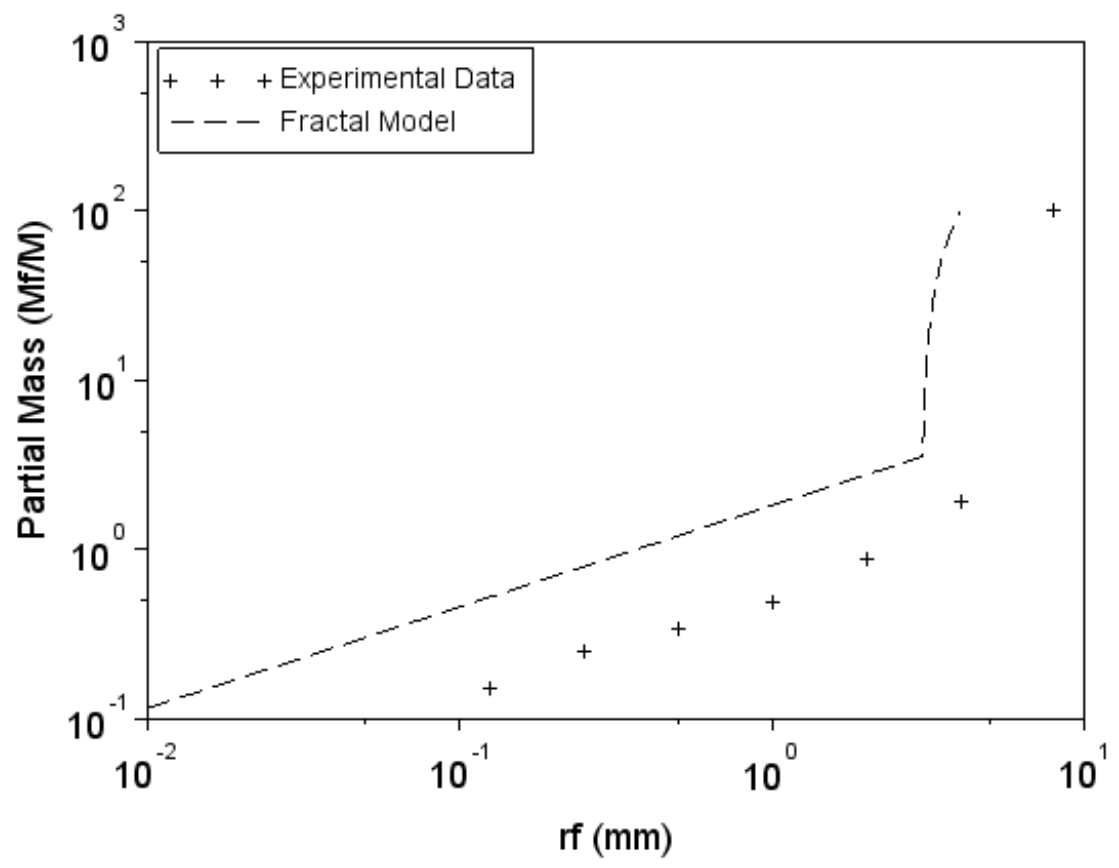

Figure 4.15: Validation separate effects test Rod 2

The partial mass expresses in percentage (\%) the cumulative mass of particles below $\mathrm{rf}$ 


\section{Bibliography}

[1] Benoît B. Mandelbrot, "Gli oggetti frattali: Forma, caso e dimensione", Biblioteca Einaudi 1987

[2] Benoît B. Mandelbrot, "The Fractal Geometry of Nature", Times Books 1982

[3] A. Carpinteri, G. Lacidogna, N. Pugno, "Scaling of energy dissipation in crushing and fragmentation: a fractal and statistical analysis based on particle size distribution", International Journal of Fracture 129: 131-139, 2004.

[4] A. Carpinteri, N. Pugno, "Fractal fragmentation theory for shape effects of quasi-brittle materials in compression", Magazine of Concrete Research, 2002, 54, No. 6, December, 473-480

[5] A. Carpinteri, N.Pugno "A multifractal comminution approach for drilling scaling laws", Powder Technology 2003

[6] M. Flanagan, P. Askeljung, "Observation of fuel fragmentation, mobility and release in integral, high-burnup, fueled LOCA tests", Presented at Enlarged Halden Program Group Meeting, Sandefjord, Norway, 2-7 October 2011E.Perfect, "Fractal models for the fragmentation of rocks and soils: a review", Engineering Geology 1997

[7] Eghball, B., Mielke, L.N.. Calve. G.A., Wilhelm," Fractal description of soil fragmentation for various tillage methods and crop sequences" Soil Sci. Sot. Am. J. 57, 1337-1341. 1993

[8] E.Perfect, B.D. Kay, J.A Ferguson, A.P. da Silva, K.A. Denholm, " Comparison of functions for characterizing the dry aggregate size distribution of tilled soil", Soil Tillage Re. 28, 123-139, 1993

[9] A.H. Thompson, "Fractal in rock physics", Ann. Rev. Earth Planet Sci 19, 237-262, 1991

[10] Donald L. Turcotte, "Fractals and Chaos in Geology and Geophysics", Cambridge Univ Pr, 1997

[11] C. G. Sammis, "Fractal fragmentation and frictional stability in granular materials", Proceedings of the IUTAM Symposium on Mechanics of Granular and Porous Materials, Kluwer Academic Publisher pp. 23-34, 1997

[12] H. Takayasu, "Fractals in the Physical Sciences", Manchester University Press New York 1990

[13] C. Brown, L. Liebovitch, "Fractal Analysis", SAGE Publications 2010

[14] P. Clifford, "Potential Impacts on Design Basis Accidents", Office of Nuclear Reactor Regulation, Public Meeting on Fuel Fragmentation, Relocation and Dispersal, March 2014

[15] D. Ginestar, R. Miro, G. Verdu, T. Barracchina, „Modal processing of the Local Power Range Monitors signals in BWR NPP", Annals of Nuclear Energy, Volume 38, Issue 11, November 2011, Pages 2441-2455

[16] A. Gruen, "PWR optimization for operational flexibility and fuel utilization", Nuclear Engineering and Design 122 (1990) 329-338

[17] J. K. Shultis, "Determining axial fuel-rod power density profiles from in-core neutron flux measurements", Nuclear Instruments and Methods in Physics Research Section A, Volume 547, Issue 2-3, August 2005, Pages 663-678

[18] R. Tayama, K. Hayashi, R. Iwasaki, M. Sasaki, Y, Etoh, H. Sakurai, "Measurement and calculation of neutron densities in BWR high burn-up fuels", Nuclear Engineering and Design 210 (2001) 239-248

[19] ANSI/ANS-5.1-2005, "Decay heat in light water reactors", American Nuclear Society 2005 
[20] D. N. Sah, U.K. Viswanathan, S. Anantharam, "Study of creep deformation of irradiated zircaloy cladding by isothermal heating of irradiated PHWR fuel pins in temperature range $700^{\circ} \mathrm{C}-$ $900^{\circ} \mathrm{C}^{\prime \prime}$, Transactions of the Indian Institute of Metals, April 2010, Volume 63, Issue 2-3, pp 467471

[21] G. Khvostov, W. Wiesenack, M.A. Zimmermann, G. Ledergerber, „Some insights into the role of axial gas flow in fuel rod behavior during the LOCA based on Halden tests and calculations with the FALCON-PSI code", Nuclear Engineering and Design, Volume 241, Issue 5, May 2011, Pages 1500-1507

[22] M.Kinoshita, T. Sonoda, S. Kitajima, A. Sasahara, T. Kameyama, T. Matsumura, E. Kolstad, V.V. Rondinella, C. Ronchi, J.P. Hiernaut, T. Wiss, F. Kinnart, E. Ejton, D.Papaioannou, H. Matze, "High-burnup rim project: (III) properties of rim-structures fuel", (Paper 1102) in: International Meeting on LWR fuel Performance, Orlando, Florida, 2004

[23] "TRANSURANUS handbook", European Commission, JRC Institute for Transuranium Elements 2011

[24] K.J. Geelhood, W.G. Luscher, J.M. Cuta, "FRAPTRAN-1.5: A computer Code for the Transient Analysis of Oxide Fuel Rods", NUREG/CR-7023, Vol. 1 Rev. 1

[25] H.G. Sonnenburg, "Fuel Rod Behaviour und Accident Condition Analyses with TESPA-ROD", EUROSAFE forum 2004

[26] B.C.Oberländer, M.Espeland, N.O.Solum, H.K.Jenssen, "LOCA IFA650-4: PIE of the high burnup (92 MWd/kgU) PWR segment", EHPG Meeting Loen, May 2008 


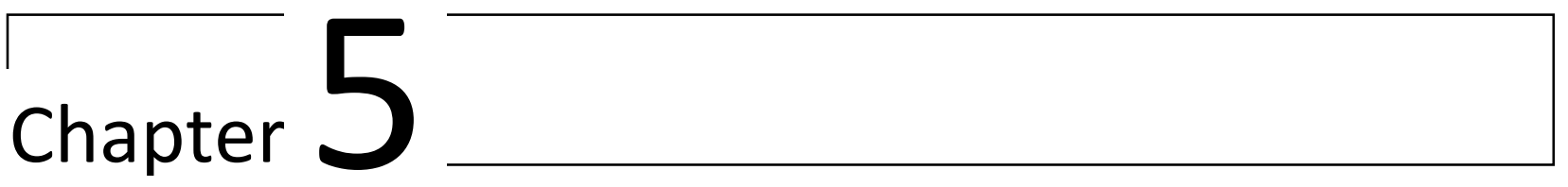

\section{Safety Significance and conclusions}

\section{Contents}

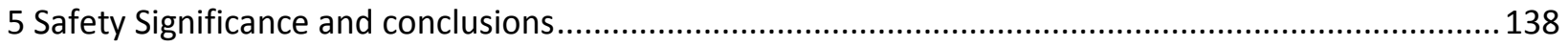

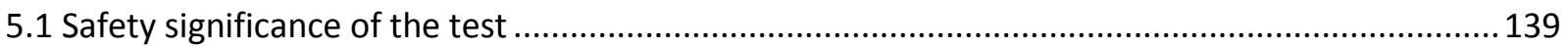

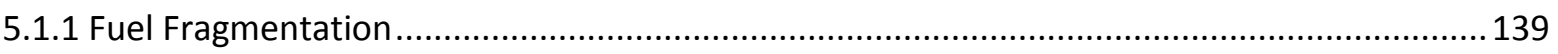

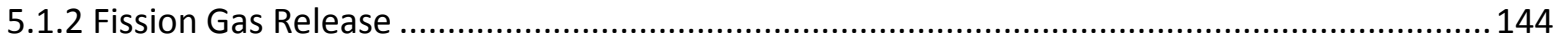

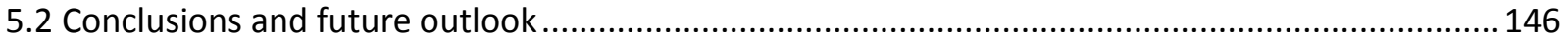

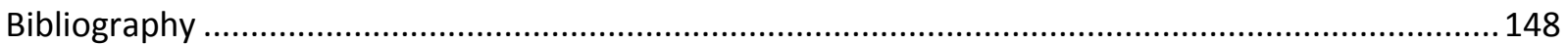

The experiment presented in this dissertation provides a simpler and cheaper way of investigating of fuel fragmentation during LOCA in comparison to the more complex experiments executed in-pile in a research reactor.

The knowledge gathered during the experiment design and the results of the separate effects test and post-experiment investigations gave the opportunity to assess the safety significance of the experimental research with regards to fuel fragmentation and fission gas release during LOCA.

As conclusion, suggestions and proposals are developed based on the lessons learned during the different phases of the experiment's execution and the knowledge gathered attempting to model the resulting fragment size distribution. 


\subsection{Safety significance of the test}

The safety significance of the test is discussed separately for fuel fragmentation and the fission gas release during the transient. There is a historical reason for this approach. The consequences of fuel fragmentation were always considered in relation to the fuel behavior during LOCA to ensure that the safety criteria specified in the Title 10 of the Code of Federal Regulations (10 CFR) 50.46 (b) "Acceptance criteria for emergency core cooling system for light-water nuclear power reactors" were met [1].

The total fission gas release during LOCA into the environment is instead a part of the consideration of the radiological effects of postulated accidents. The issue has been historically complex and it has been subject to considerable evolution in the regulatory review process, in particular in the US [2]. The 10 CFR Part 50 [3] and 10 CFR Part 52 "Licenses, Certifications, and Approvals for Nuclear Power Plants" [4] requires an evaluation of the radiological release that would result from the operation of a nuclear facility in order to assess the risk to public health and safety. The issue is also connected with the criteria of licensing of a specific site (10 CFT Part 100 "Reactor Site Criteria" [5]) for the determination of the exclusion area, the low population zone and the population centre distance. The determination of these areas shall assume in the calculation an appreciable release of quantities of fission products following a major accident such as a substantial meltdown. This regulatory background of the radiological aspects of plant safety analysis shows the complexity and how very severe conditions are considered, far beyond a LOCA event terminated with the prompt function of the ECCS as studied in this dissertation. The safety significance of the test in terms of fission gas release was therefore evaluated considering more specific guidelines and specificities in different regulatory approaches.

In 3.3.3 it was underlined the effect fission gas release may have on the fuel behaviour during the transient, particularly regarding cladding deformation. Consequently, a future coherent integration of FGR considerations on fuel behaviour during LOCA is expected.

\subsubsection{Fuel Fragmentation}

The safety significance assessment of the separate effects test starts with the following two safety considerations in relation with the main fuel fragmentation's concerns: fragments relocation in the ballooned region and fuel dispersal.

- Safety consideration 1: it has been confirmed that with a fuel at approximately $52 \mathrm{MWd} / \mathrm{kgU}$ subject to LOCA, the fuel axial relocation is negligible with a negligible effect on the decay heat removal in the ballooned region.

- Safety consideration 2: it has been confirmed that with a fuel at approximately $52 \mathrm{MWd} / \mathrm{kgU}$ subject to LOCA, fuel dispersal is negligible because fuel fragments are too big to exit through the burst opening with a width of $0.3 \mathrm{~mm}$. 
It should be underlined that the proposed fractal law and its way of representation, such as Figure 4.15 for Rod 2, are very appropriate to estimate the potential fuel dispersal from a rod that experiences burst. Figure 5.1 includes Figure 4.15 and the estimated opening size width for Rod 2 of $0.3 \mathrm{~mm}$ (3.3.2). An evaluation of fuel dispersal can be made estimating the amount of particles smaller than the opening size. Even considering the fractal model that is more conservative with respect to the experimental data, the potential of fuel dispersal is estimated to be below $1 \%$.

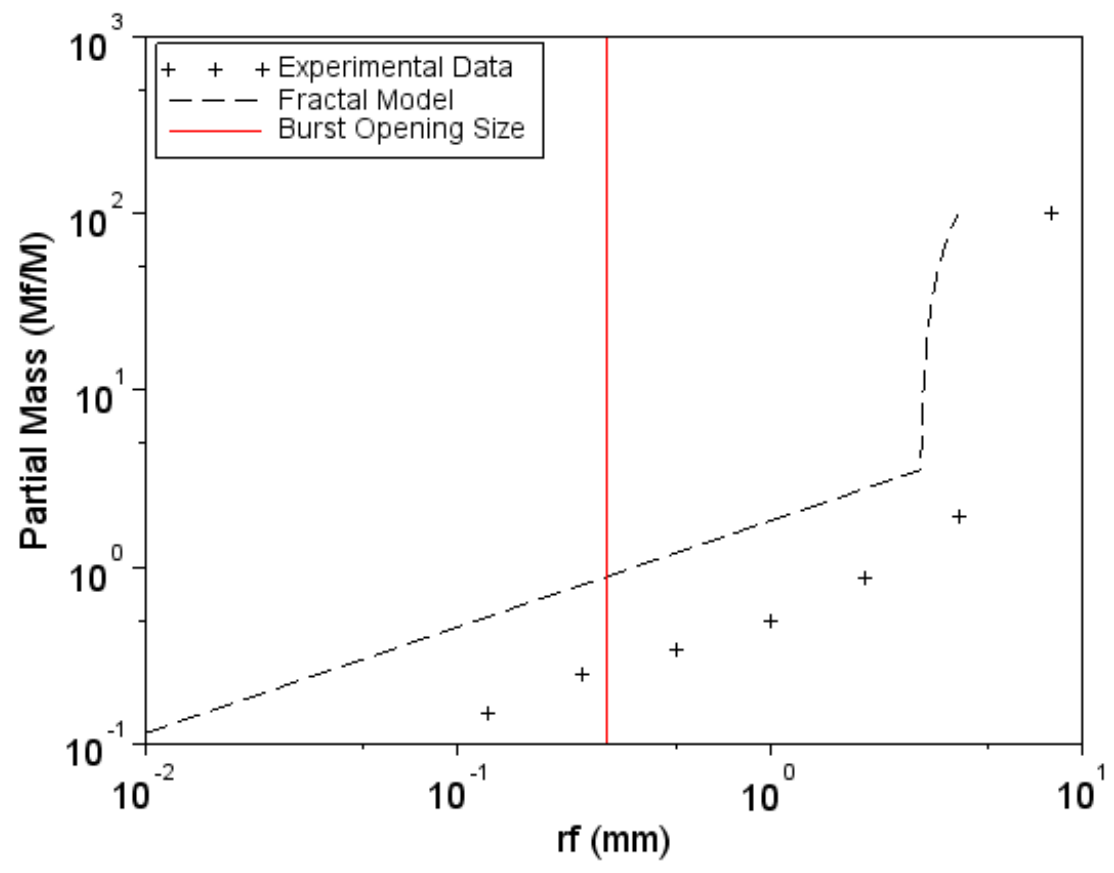

Figure 5.1: Estimation of potential fuel dispersal for Rod 2

The partial mass expresses in percentage (\%) the cumulative mass of particles below $\mathrm{rf}$

The experiment's outcomes and the developed fractal law with determination of fractal law coefficients provide guidance on the qualitative interpretation of previous experiments that show strong fuel fragmentation and dispersal and raised concern in the nuclear fuel community. Owing to similarities in the fuel burnup of the separate effect test ( $52 \mathrm{MWd} / \mathrm{kgU})$, the US NRC Test 198 gives the opportunity to discuss the differences in view also of a real core reactor scenario. The test was performed at the Studsvik laboratories in Sweden and resulted in rod ballooning and burst. Table 5.1 summarizes the main characteristics of the US NRC 198 Test based on the data from [6]

\begin{tabular}{|c|c|}
\hline Parameter & Value \\
\hline Burnup $(\mathrm{MWd} / \mathrm{kgU})$ & 52 \\
\hline $\mathrm{PCT}\left({ }^{\circ} \mathrm{C}\right)$ & 1160 \\
\hline Fill in pressure (bar) & 82 \\
\hline Burst Pressure (bar) & 74 \\
\hline Rupture Temperature $\left({ }^{\circ} \mathrm{C}\right)$ & 693 \\
\hline
\end{tabular}

Table 5.1: US NRC Test 198 characteristics 
Comparing the neutron radiography results of Rod 2 (ballooning and burst) with the visual inspection of Test 198 as shown in Figure 5.2, it is possible to make the following considerations:

- Consideration 1: The fuel fragmentation of Rod 2 is limited to some fuel pellets very close to the ballooning and burst region. The fragmentation observed in this region seems to be comparable to the visual inspection of the US NRC Test 198.

- Consideration 2: The neutron radiography shows also that axial regions away from the ballooning and burst do not show fuel fragmentation, although some cracks were not detected by the neutron radiography. The number of pellets that experience fuel fragmentation in test 198 seems much bigger.
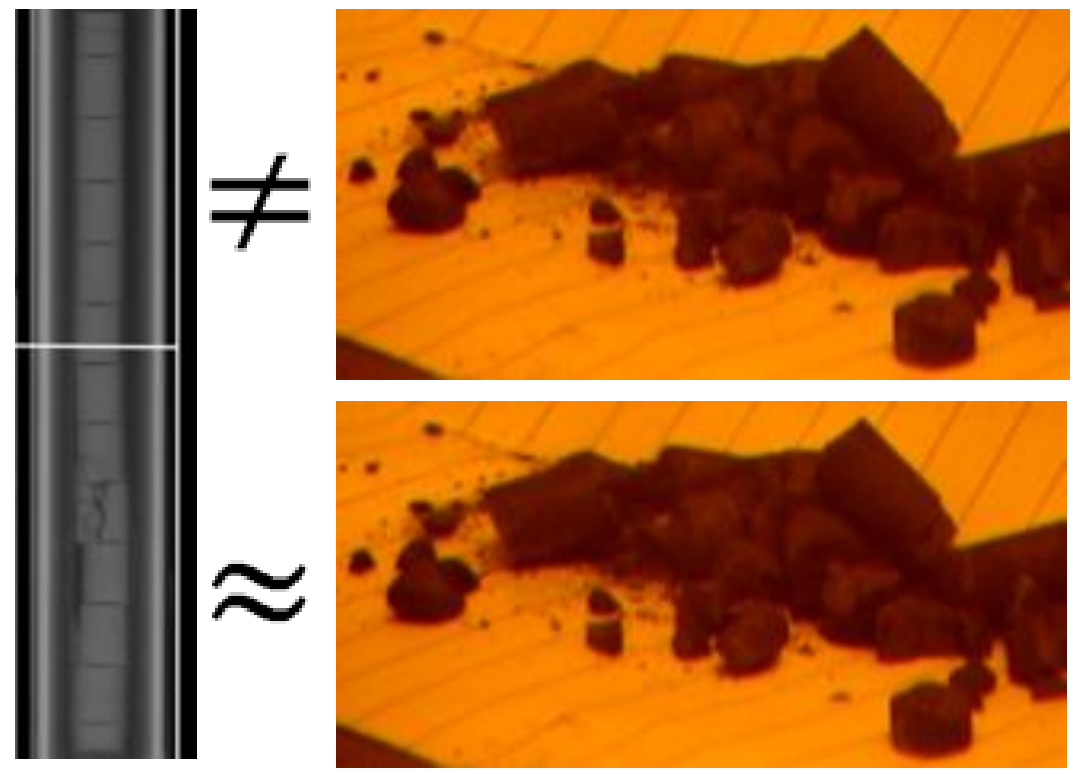

Figure 5.2: Comparison neutron radiography Rod 2 and visual inspection NRC 198

Interpreting the two considerations above with the fractal law developed in Chapter 4, it can be concluded that the value of $r_{f, \min 1}$ is comparable, because in the burst region the fragmentation is comparable, but in the US NRC test 198, the value of $K$ is much bigger because a bigger mass takes part in the fragmentation process with small particles. In other words, a bigger number of pellets are involved in the fragmentation process. Owing to an identified difference in the $K$ coefficient and considering a very similar fuel burnup, the reasons of the discrepancies should be found in a different degree of removal of the constraint provided by the rod inner pressure and/or of the geometrical constraint provided by the cladding.

Figure 5.3 shows a comparison between the inner rod pressure transient calculated by TRANSURANUS for Rod 2 and the inner pressure rod transient for the NRC Test 198. The test procedure at Studsvik implied a fill-in pressure of 82 bar that was kept constant until the fuel reached $300{ }^{\circ} \mathrm{C}$ and burst 
occurred at a pressure value slightly smaller than 74 bar (see Table 5.1). The burst difference between the two tests is estimated to be approximately 34 bar and based on how the burst effect was considered in the formulation of $K$ in 4.2, this is a first contribution in determining a bigger number of fuel mass participating in the fragmentation process for Test 198.

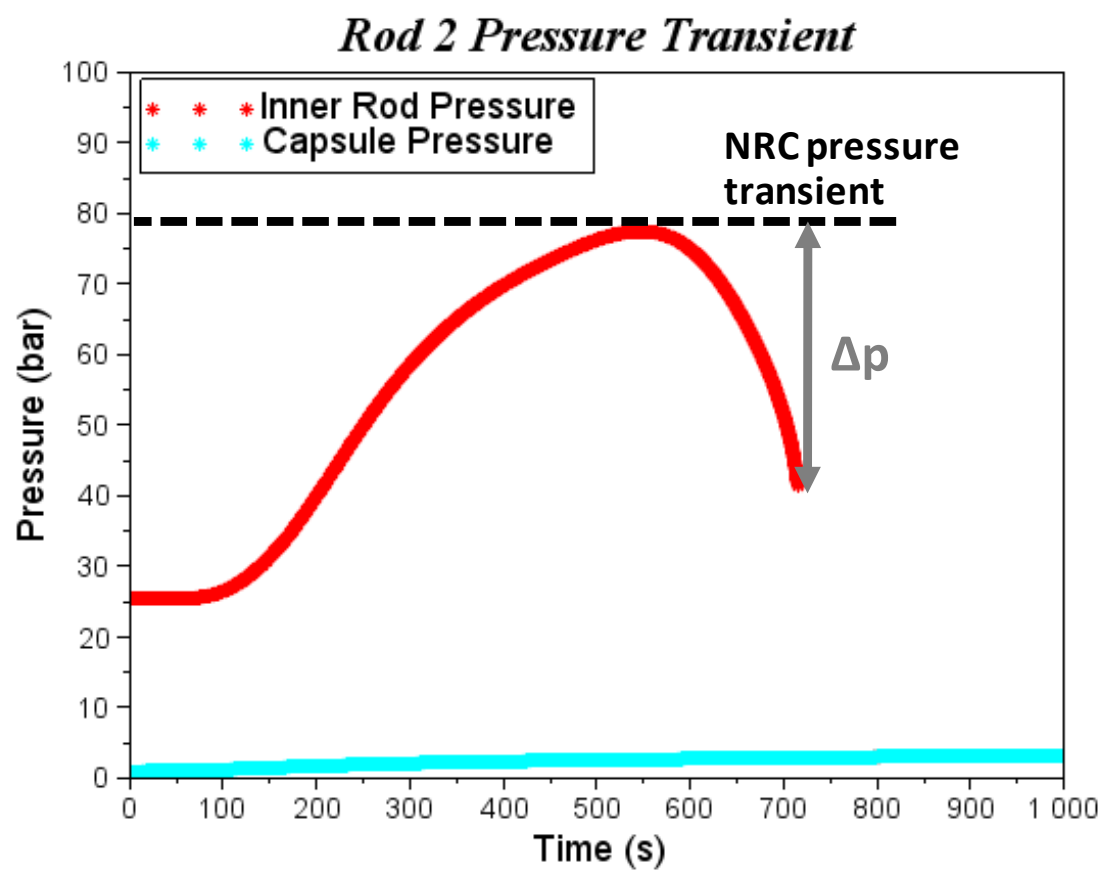

Figure 5.3: Comparison of rod pressure transient Rod 2 and NRC 198

Figure 5.4 is taken from [7] and it shows the cladding circumferential strain of Test 198. The cladding deformation of Rod 2 is shown in Figure 3.27. To perform a detailed comparison of the average cladding circumferential strain, a complete set of data from Test 198 would have been required and they were not available. However, already a qualitative comparison of the two plots shows a bigger cladding deformation for Test 198 that was also driven by the relatively high value of pressure inside the rod. Another variable that increased the cladding deformation was the lack of flexibility at the bottom and top of the rod to allow cladding elongation. The developed separate effects test instead allowed rod elongation because of the design of the rod/capsule bar connection at the bottom and top of the rod showed in Figure 2.15. Considering the resulting difference in the average cladding circumferential strain and based on how the removal of the geometrical constraint of the cladding was considered in the formulation of $K$ in 4.2, this is a second contribution in determining a bigger number of fuel mass participating in the fragmentation process for Test 198. 


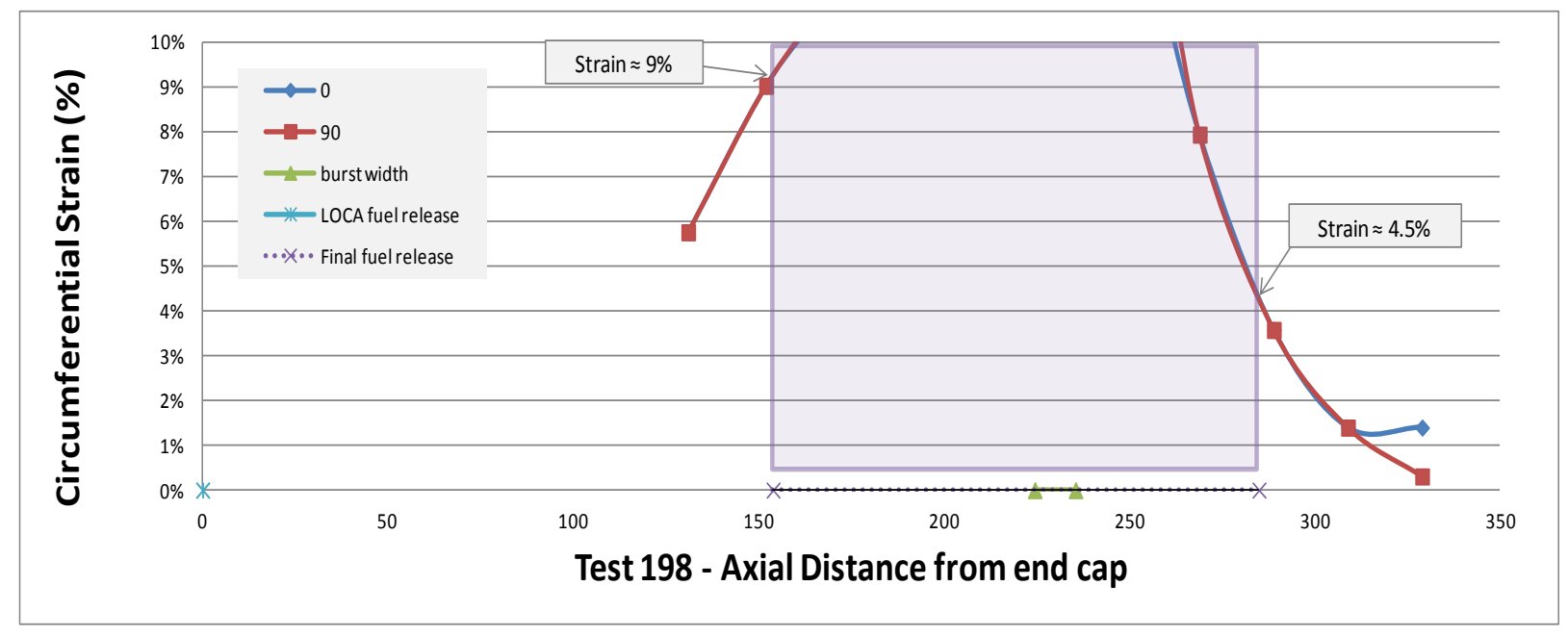

Figure 5.4: Post-test cladding circumferential strain for US NRC Test 198 Figure from Reference [7]

The considerations of above are not only listed to explain the discrepancies between two tests at similar burnup but also to underline how experiment's features may influence the outcomes of a test and therefore how their significance in terms of specific safety criteria should be filtered.

Considering a full length rod of a commercial reactor condition, the cladding average circumferential strain is limited by the spacer grids and by the configuration of the fuel assembly made of a set of fuel rods with a small distance between the rods. Typical values of rod-to-rod pitches are $0.496 \mathrm{~cm}$ in a $17 \times 17$ PWR fuel bundle and $0.561 \mathrm{~cm}$ in a 9x9 BWR fuel bundle. The test at the Pacific Northwest Laboratory in Canada was the first series of test performed on a bundle of rods instead of a single rod [8, $9,10,11$ and 12]. The outcomes of these tests show that in a bundle of rods, ballooning can occur such that all the balloons are coplanar and this limits the cladding circumferential strain having a positive effect on the constraint provided by the cladding. The coplanar ballooning is shown in Figure 5.5. An additional positive effect of the Canadian investigation in retaining the constraint given by the cladding was the evidence that ballooning is mostly prevented in the sections of the fuel rods inside a grid spacer as it would occur in a full length rod of a commercial power reactor. 


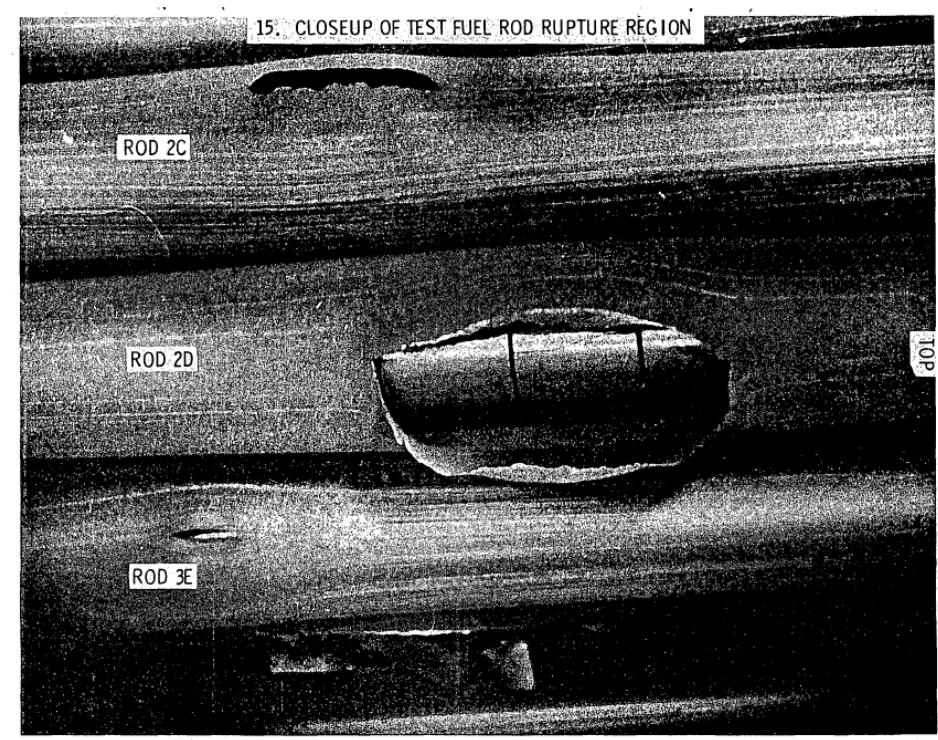

FIGURE 5.15. Closeup of Test Fuel Rod Rupture Region

Figure 5.5: Coplanar ballooning in LOCA test at Pacific Northwest Laboratory

Figure from Reference [2]

Considering the effect of burst in full length fuel rods, it should be underlined that, differently from the NRC Test 198, the level of burst pressure in the high burnup fuel rod should be substantially smaller. This is connected to the level of communication between axial regions of the fuel. The axial communication is weakened because of the gap closure and fuel/cladding bonding. This effect was studied in [13] and it is believed to be able to amplify the predicted dynamic effects of gas nonuniformity during the LOCA. The pressure in the balloon region in fuel rods with bonding has a larger decrease of pressure because the balloon region is as "axially isolated". This phenomenon brings also to a longer transient duration before that the burst criteria are met and burst occurs at lower pressure. This phenomenon has also a positive effect in reducing fuel fragmentation based on the burst effect studied in this dissertation.

The safety significance of the test was assessed against experiments with similar burnup and studying the potential boundary conditions of full length fuel rods with the support of other experiments and research. It was concluded that for a full length rod, there is a bigger retention of the geometrical constraint given by the cladding and a reduced effect given by rod burst with a consequent positive effects in terms of degree of fuel fragmentation.

\subsubsection{Fission Gas Release}

The safety significance of the separate effects test regarding the fission gas release was analyized considering specific regulatory guides and specificities in different Countries. 
In terms of specific LOCA regulations for evaluating the radiological consequences of a LOCA, in the US the reference guidelines are Regulatory Guide 1.3 Revision 2, "Assumptions Used for Evaluating the Potential Radiological Consequences of a Loss of Coolant Accident for Boiling Water Reactors" [14], and Regulatory Guide 1.4 Revision 2, "Assumptions Used for Evaluating the Potential Radiological Consequences of a Loss of Coolant Accident for Pressurized Water Reactors" [15], both state the following:

- Twenty-five percent of the equilibrium radioactive iodine inventory developed from maximum full power operation of the core should be assumed to be immediately available for leakage from the primary reactor containment.

- One hundred percent of the equilibrium radioactive noble gas inventory developed from maximum full power operation of the core should be assumed to be immediately available for leakage from the reactor containment.

In the US, these regulatory guides assume that all the noble gases have escaped from the fuel, the fuel rod cladding and the reactor coolant system and therefore available for leakage from the reactor containment. These regulatory criteria assume therefore that all the fuel rods in the core rupture and the noble gases are released completely.

The safety assessment of the separate effects test performed with a $52 \mathrm{MWd} / \mathrm{kgU}$ shows that only $7.3 \%$ of noble gases are released during the LOCA with a big margin to the $100 \%$ release assumed by the US NRC Regulatory Guides 1.3 and 1.4. Additionally, not all the fuel rods are expected to burst.

A different regulatory approach exists in Germany. The RSK-Leitlinien für Druckwasserreaktoren prescribes a maximum failure rate of the fuel rods of the core of $10 \%$ and one hundred percent of noble gases to be considered released from these rods for the reactor containment evaluation. An evaluation performed in [16] showing a cycle analysis performed for a German PWR calculated that only 3.7\% of all rods in the core failed. The analysis was performed with a slightly reduced degree of conservatism with respect to axial power redistribution and fuel rod data. In particular, for each individual rod a heat-up calculation is performed based on the actual fuel rod condition and a conservative deterministic thermal-hydraulics boundary conditions to determine whether the rod would fail or not.

Discussions on the safety significance of the fission gas release does not have the same attention compared to fuel fragmentation because of the $100 \%$ conservatism in the evaluation of the performance of the reactor containment and the radiological consequences at site. However if the number of burst rods is limited, as in Germany, the transient fission gas release may also lead to a higher number of burst rods. One has to keep in mind that the German failure rate calculations are already done with a great number of conservative assumptions. In consequence the transient fission gas release may only have an effect on the best-estimate calculation results which are not relevant from a regulatory point of view. Nevertheless fuel performance codes should improve the model of the fission gas release during the transient in order to better understand the consequences and further research is needed to collect additional data points for models' validation. 


\subsection{Conclusions and future outlook}

The experimental investigation presented with this work provides an alternative way to study the behavior of fuel during LOCA. The preparatory work needed to design the experiment constitutes a strong basis from which starting a new experimental campaign for the following reasons:

- Reference test: the separate effect experiment is a successful reference test and it is supported by validated design tools that are ready for use.

- Experimental Rig: The experimental rig structure made of capsules as containment of fuel rods allows the study of fuel behavior of a similar heating up varying other boundary conditions. Possible variables to study are:

- Burnup: Fuel segments with similar rod parameters such as length, upper free volume and fill-in pressure but with different burnup to evaluate its effect freezing other boundary conditions.

- Burst Pressure: Fuel segments with similar burnup but different fuel rod parameters such as upper free volume and fill-in pressure with the objective to meet the burst criteria at different inner rod pressure to study the influence of different degrees of removal of the constraint given by the rod internal pressure.

- Ballooning size: Fuel segments with similar burnup but different fuel rod parameters such as upper free volume and fill-in pressure with the objective to achieve different ballooning size to study more in detail the effect of removal of the geometrical constraint provided by the cladding at different stages.

- Heating ups: The combinations of above can be studied also with successive runs to estimate the influence of the heating up rate on fuel fragmentation. Considering a specific fuel burnup, an appropriate heating up rate can be applied to simulate a realistic core scenario that takes into account the LHGR as function of the fuel burnup and transient characteristics (e.g. PCT and transient duration) as function of the location of the associate fuel rod assembly in the core.

- Capsule Design: the capsule allows the collection of the fission gas released from the fuel from burst rods with opportunities of specific and more detailed studies on fission gas release.

Improvements of the experimental design to be able to add value to research on fuel fragmentation during LOCA have been also identified and they can be implemented in a further experimental campaign:

- Heating up: The identified experimental procedure implied a target temperature of the oven fixed at $900{ }^{\circ} \mathrm{C}$. The temperature of the oven can be varied to follow a specific fuel rod cladding transient or for instance to increase the initial ramping up of the capsule's temperature just by increasing the initial target temperature of the oven to improve the heat flux from the oven to the capsule and from the capsule to the rod. 
- Pressure monitoring: The current configuration allows the monitoring of the capsule pressure to detect the burst time and to determine the integral value of the fission gas release during the transient. The experimental rig can be improved with monitoring of the pressure of the rod to determine the transient fission gas release because the small upper free volume and an accurate measure of temperature will allow the calculation of this value as function of time.

- Fragmentation monitoring: The neutron radiography performed immediately after the LOCA test provides good information about the resulted fuel fragmentation. The information that is missing is when over the course of the transient the fragmentation develops. Two approaches can be investigated: a microphone installed inside the capsule that capture any sounds coming from the rod or an infrared camera to be positioned inside the oven to try to obtain information on fuel fragmentation during the transient.

These recommendations are given because further research is planned to be done on fuel fragmentation. This topic has been having an enormous international attention. Prove of that are the Public Meeting scheduled by the US NRC on "Fuel Fragmentation Relocation and Dispersal" held in Washington in March 2014, the established task group of the OECD/NEA "Task Group on Fuel Fragmentation, Relocation and Dispersal during LOCA" and the intention of the IAEA project on "Fuel Modeling in accident conditions, FUMAC" to be started in 2015.

Two objectives are primarily identified by these working groups:

1 The need of additional experimental data to study fuel fragmentation and fission gas release during LOCA

2 The need to improve the modeling of fuel fragmentation and the fission gas release in fuel performance codes to use these models in the evaluation of LOCA in safety analysis of nuclear reactors.

The work performed in the Thesis started in 2011 and it was precursory in identifying these two needs. Effort was made to design an alternative experiment to separate the effects that played a major role in determining fuel fragmentation and fission gas release during LOCA and an attempt of modeling of the phenomena was made. The work was performed with passion and integrity and it was driven by the mission of a continuous safety assessment of the operation of nuclear power plants. 


\section{Bibliography}

[1] U. S. Code of Federal Regulations, "Domestic Licensing of Production and Utilization Facilities," Part 50, Chapter I, Title 10, "Energy."

[2] Patrick A.C. Raynaud, "Fuel Fragmentation, Relocation and Dispersal During the Loss-of-coolant Accident", NUREG-2121, US NRC 2012.

[3] U.S. Code of Federal Regulations, "Acceptance Criteria for Emergency Core Cooling Systems for Light-Water Nuclear Power Reactors," Title 10, Part 50, Section 46, January 1974 (amended).

[4] U.S. Code of Federal Regulations, "Licenses, Certifications, and Approvals for Nuclear Power Plants," Part 52, Chapter I, Title 10, "Energy."

[5] U.S. Code of Federal Regulations, "Reactor Site Criteria," Part 100, Chapter I, Title 10, "Energy."

[6] M. Flanagan, P. Askeljung, "Observation of fuel fragmentation, mobility and release in integral, high-burnup, fueled LOCA tests", Presented at Enlarged Halden Program Group Meeting, Sandefjord, Norway, 2-7 October 2011

[7] M. Flanagan, P. Askeljung, "Observations of Fuel Fragmentation, Mobility and Loss in Integral, High-Burnup, Fueled LOCA Tests", Halden Workshop on LOCA, Lyon, France, May 2012

[8] G.E. Russcher et al., "LOCA Simulation in the NRU Reactor: Materials Test-1,"NUREG/CR-2152, U.S. Nuclear Regulatory Commission, Washington, DC, October 1981. ADAMS Accession No. ML083360603.

[9] 23. J.O. Barner et al., "Materials Test-2 LOCA Simulation in the NRU Reactor," NUREG/CR-2509, U.S. Nuclear Regulatory Commission, Washington, DC, March 1982.ADAMS Accession No. ML101970158.

[10] 24. C.L. Mohr et al., "LOCA Simulation in the National Research Universal Reactor Program: Data Report for the Third Materials Experiment (MT-3)," NUREG/CR-2528, U.S. Nuclear Regulatory Commission, Washington, DC, April 1983. ADAMS Accession No. ML083360587.

[11] 25. C.L. Wilson et al., "LOCA Simulation in NRU Program: Data Report for the Fourth Materials Experiment (MT-4)," NUREG/CR-3272, U.S. Nuclear Regulatory Commission, Washington, DC, July 1983. ADAMS Accession No. ML101960140.

[12] 26. M.D. Freshley and G.M. Hesson, "Summary Results of the LOCA Simulation Program Conducted in NRU", Report PNL-SA-11536, October 1983.

[13] G. Khvostov, W. Wiesenack, M.A. Zimmermann, G. Ledergerber, „Some insights into the role of axial gas flow in fuel rod behavior during the LOCA based on Halden tests and calculations with the FALCON-PSI code", Nuclear Engineering and Design, Volume 241, Issue 5, May 2011, Pages 1500-1507

[14] U.S. Nuclear Regulatory Commission, Regulatory Guide 1.3, "Assumptions Used for Evaluating the Potential Radiological Consequences of a Loss of Coolant Accident for Boiling Water Reactors," Revision 2, Washington, DC, June 1974.

[15] U.S. Nuclear Regulatory Commission, Regulatory Guide 1.4, “Assumptions Used for Evaluating the Potential Radiological Consequences of a Loss of Coolant Accident for Pressurized Water Reactors," Revision 2, Washington, DC, June 1974. 
[16] A. Wensauer, "LOCA: an operator's view on German licensing practice", Fuel Behaviour and Modelling under Severe Transient and Loss of Coolant Accident (LOCA) Conditions. Proceedings of a Technical Meeting

[17] "RSK-Leitlinien für Druckwasserreaktoren", RSK-Leitlinien, Ursprungsfassung (3. Ausgabe vom 14. Oktober 1981) mit Änderungen vom 15.11.1996 


\section{Acknowledgements}

I would like to acknowledge E.ON Kernkraft GmbH for financing the experiment through E.ON Innovation \& Technology. It was a unique chance and I would like to extend my gratitude to Dr. Marcus Seidl who has technically supervised the work and for his inspiring guidance, Frank Hirsch for his continuous and generous support and Dr. Wolfgang Faber for his invaluably constructive criticism and advice during the project.

I express my warm thanks to Dr. Carlo Vitanza for his friendship, for his illuminating view in helping me to select a topic of such international resonance and for his kindness and generosity during my stay in Norway. I am using this opportunity to thank the team of the Halden Reactor Project for the successful execution of the experiment, particularly Dr. Margaret McGrath and Dr. Wolfgang Wiesenack.

I would like to express my sincere gratitude to my advisor Prof. Dr. Rafael Macián-Juan for the continuous support of my PhD study and research, for his patience, motivation and enthusiasm.

A very special thanks to my family Mamma, Papà e Armando, a continuous source of encouragement; I would not have made it this far without them. 